Supporting Information

\title{
Visible-Light-Induced Palladium-Catalyzed Selective Defluoroarylation of Trifluoromethylarenes with Arylboronic Acids
}

\author{
Yun-Cheng Luo, ${ }^{\dagger}$ Fei-Fei Tong, ${ }^{\dagger}$ Yanxia Zhang ${ }^{\dagger}$, Chun-Yang He, ${ }^{\ddagger}$ and Xingang Zhang*, ${ }^{\dagger}$
}

${ }^{\dagger}$ Key Laboratory of Organofluorine Chemistry, Center for Excellence in Molecular Synthesis, Shanghai Institute of Organic Chemistry, University of Chinese Academy of Sciences, Chinese Academy of Sciences, 345 Lingling Road, Shanghai 200032, China.

${ }^{\ddagger}$ Key Laboratory of Biocatalysis \& Chiral Drug Synthesis of Guizhou Province, Generic Drug Research Center of Guizhou Province, Zunyi Medical University, Zunyi, Guizhou, 563003, China 
Table of Contents

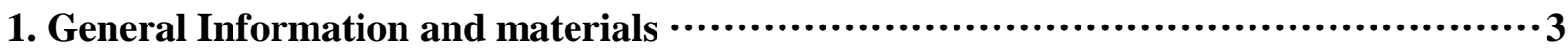

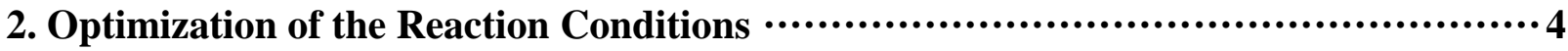

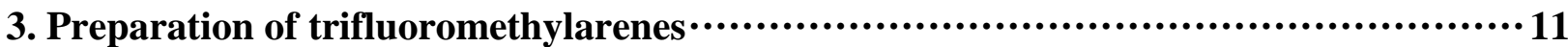

4. Visible-light-induced palladium-catalyzed selective defluoroarylation of

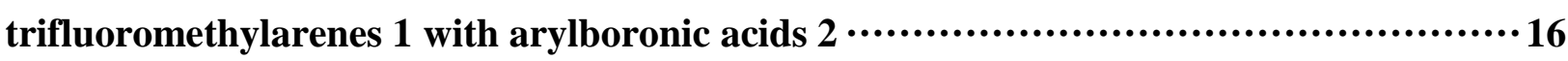

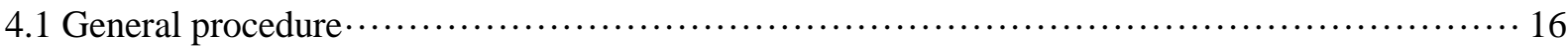

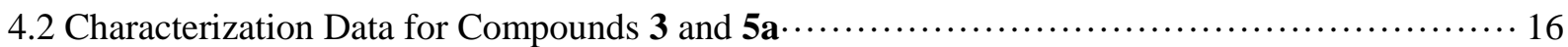

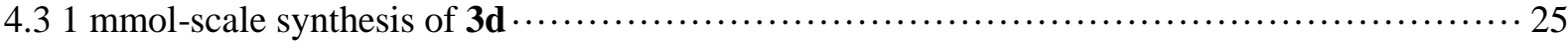

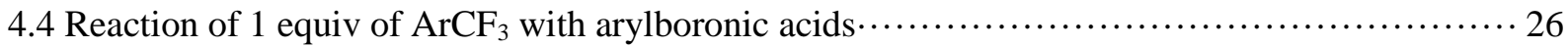

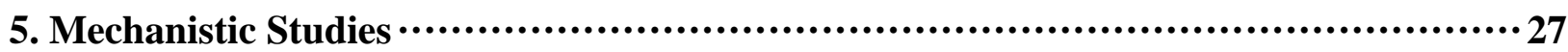

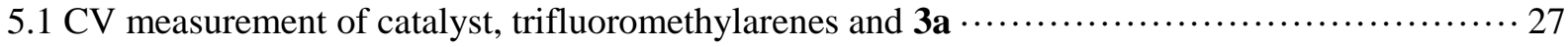

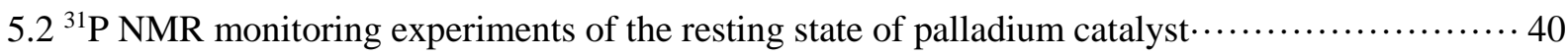

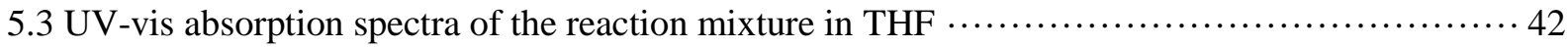

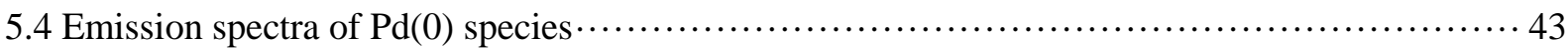

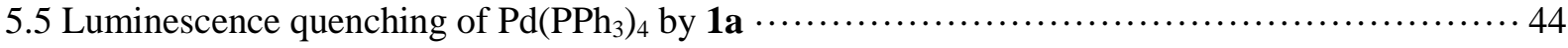

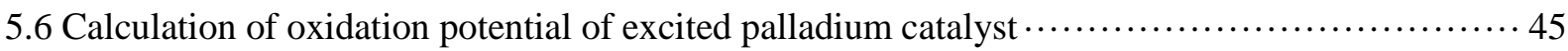

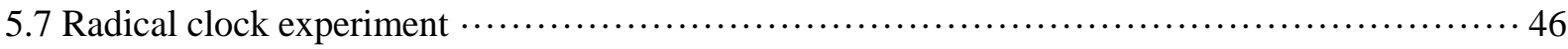

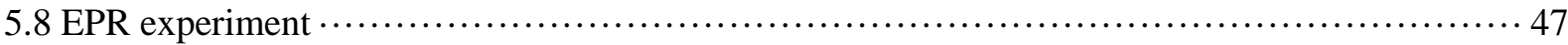

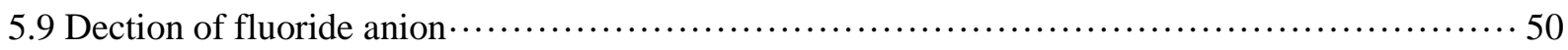

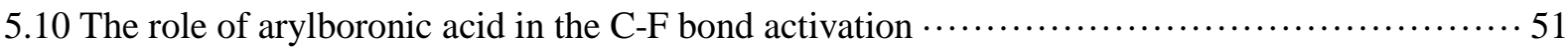

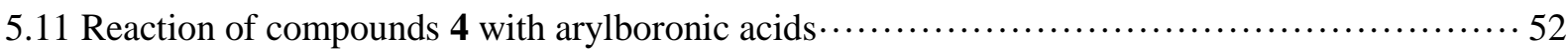

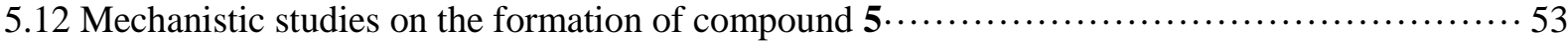

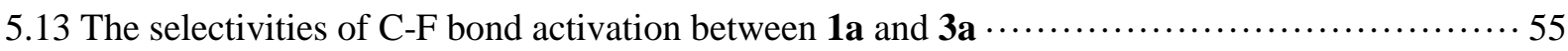

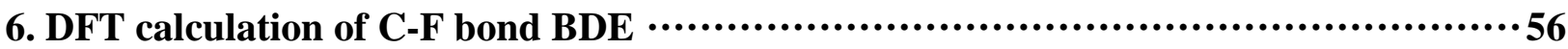

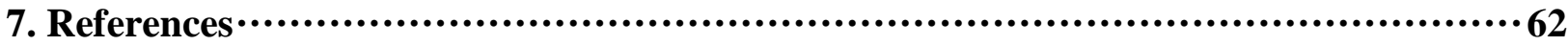

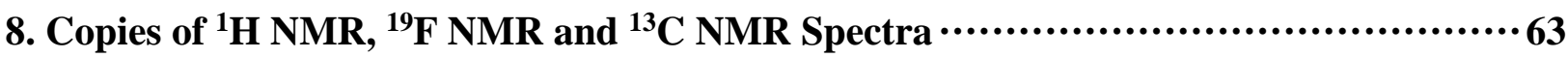




\section{General Information and materials}

General information: ${ }^{1} \mathrm{H}$ NMR, ${ }^{19} \mathrm{~F}$ NMR and ${ }^{13} \mathrm{C}$ NMR spectra were recorded on the Agilent MR 400, Bruker MR 500 and Bruker MR 600 spectrometer, and are calibrated using residual undeuterated solvent $\left(\mathrm{CHCl}_{3}\right.$ at $7.26 \mathrm{ppm}{ }^{1} \mathrm{H} \mathrm{NMR} ; 77.0 \mathrm{ppm}{ }^{13} \mathrm{C} \mathrm{NMR} ; \mathrm{CFCl}_{3}$ as an external standard and low field is positive). Chemical shifts $(\delta)$ are reported in ppm and coupling constants $(J)$ are in Hertz $(\mathrm{Hz})$. The following abbreviations were used to explain the multiplicities: $\mathrm{s}=$ singlet, $\mathrm{d}=$ doublet, $\mathrm{t}=$ triplet, $\mathrm{q}=$ quartet, $\mathrm{m}=$ multiplet, $\mathrm{br}=$ broad. NMR yield was determined by ${ }^{19} \mathrm{~F}$ NMR using fluorobenzene as an internal standard before working up the reaction. UV-vis absorption spectra were recorded on an UV 2700. Emission spectra were recorded on a Hitachi F-4600. The reverse-phase preparative HPLC used XBride Prep C18 $5 \mu \mathrm{m}$ OBD $^{\mathrm{TM}}$ 19×250 mm Column (rate: 10 mL/min). High Resolution Mass spectral data were recorded on Agilent Technologies 7250 GC QTOF spectrometer in EI mode, or on Agilent Technologies 6224 TOF LC MS spectrometer in ESI mode. IR spectra were recorded on a Bruker TENSOR 27 FTIR Spectrometer equipped with a Platinum ATR detector.

Materials: All reagents were used as received from commercial sources or prepared as described in the literature. The solvent THF was not superdry, which contain $1500-3000$ ppm water $(2.7-5.4 \mathrm{mg}$ per mL THF). $12 \mathrm{~W}$ blue LED strips (GreeThink 12V-5050-60; $1 \mathrm{~m} \times 12.5 \mathrm{~mm} \times 4.4 \mathrm{~mm}$ ) was purchased from Taobao.com. 


\section{Optimization of the Reaction Conditions}

To a $25 \mathrm{~mL}$ of Schlenk tube were added Pd catalyst, ligand, and $\operatorname{ArB}(\mathrm{OR})_{2} \mathbf{2}$ in the air. Base was added in the glovebox. The reaction mixture was then evacuated and backfilled with Ar (3 times). Trifluoromethylarene $1 \mathbf{a}$ and solvent $(2.0 \mathrm{~mL})$ was then added. After pre-stirring at $65{ }^{\circ} \mathrm{C}$ for $20 \mathrm{~min}$, the reaction mixture was irradiated with blue LED strip(s) $(12 \mathrm{~W} \times 1$ or 2$)$ for $12-24 \mathrm{~h}$ at $50-65^{\circ} \mathrm{C}$. The reaction mixture was then cooled to room temperature, and the yield was determined by ${ }^{19} \mathrm{~F} \mathrm{NMR}$ using fluorobenzene as an internal standard.

Table S1. Screening of the palladium sources ${ }^{a}$

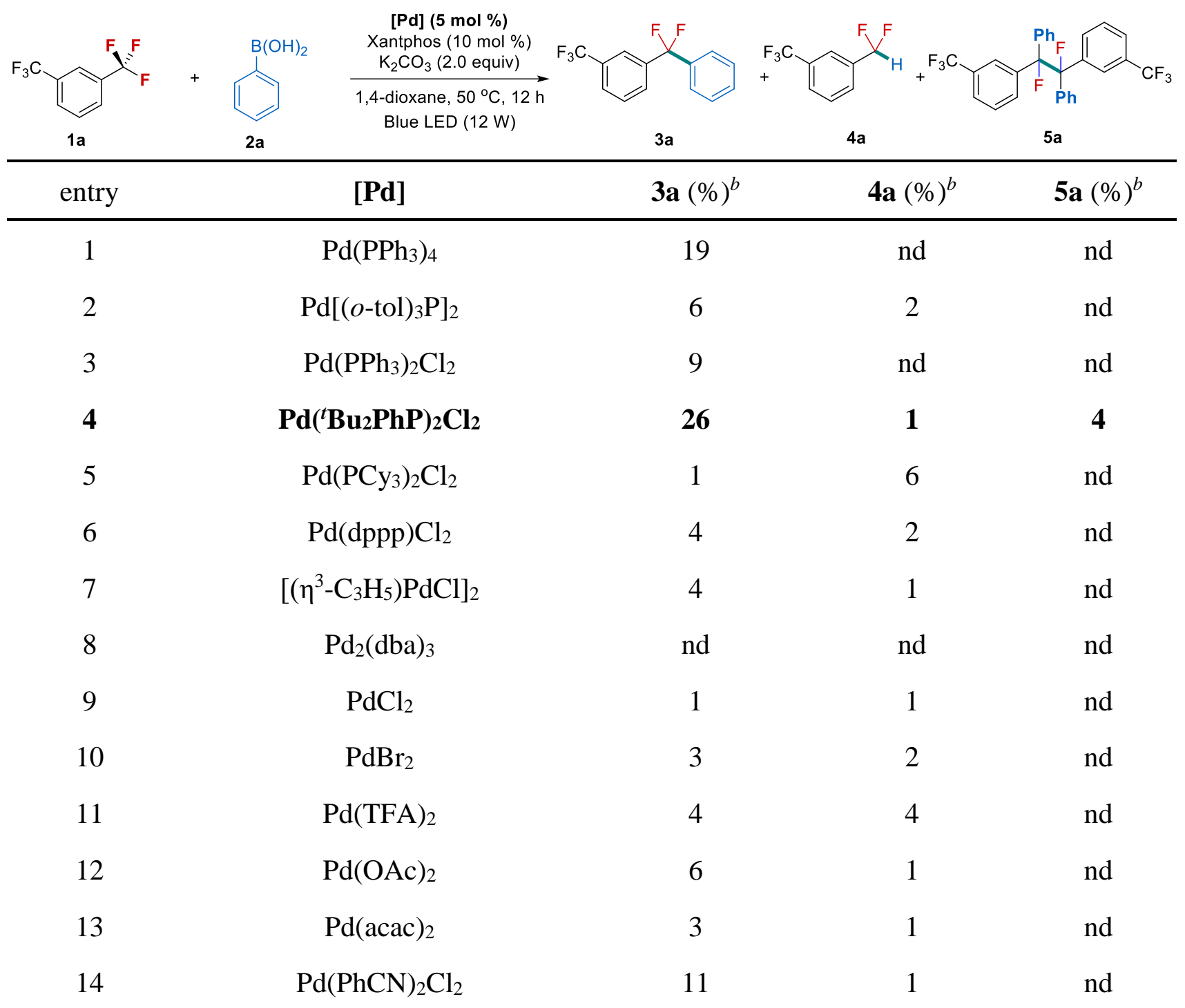

${ }^{a}$ Reaction conditions (unless otherwise specified): $1 \mathbf{a}(0.3 \mathrm{mmol}, 1.0$ equiv), 2a (2.0 equiv). The yield was determined by ${ }^{19} \mathrm{~F}$ NMR using fluorobenzene as an internal standard. ${ }^{b}$ The yield was calculated based on 1a. nd, not detected. 
Table S2. Screening of the phenylborons ${ }^{a}$

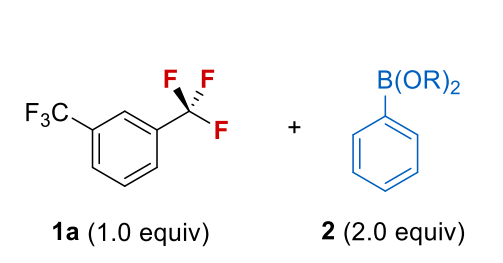

$\mathrm{Pd}\left({ }^{\mathrm{t} B \mathrm{Bu}_{2}} \mathrm{PhP}\right)_{2} \mathrm{Cl}_{2}(5 \mathrm{~mol} \%)$

Xantphos (10 mol \%)

$\mathrm{K}_{2} \mathrm{CO}_{3}$ (2.0 equiv)

1,4-dioxane, $50^{\circ} \mathrm{C}, 12 \mathrm{~h}$

Blue LED (12 W)

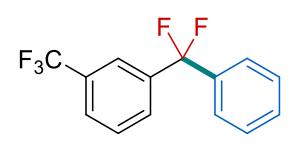

3a +

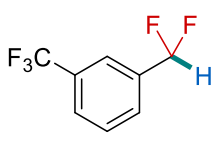

$4 a$

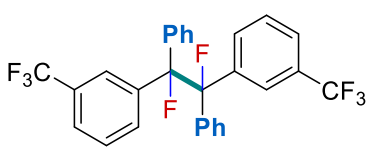

$5 a$

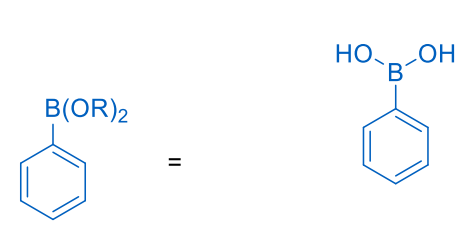

$2 a$

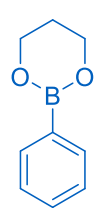

2ab

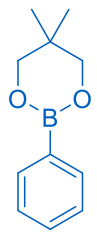

2ac

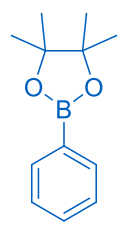

2ad

\begin{tabular}{ccccc}
\hline entry & $\operatorname{PhB}(\mathrm{OR})_{2}, \mathbf{2}$ & $\mathbf{3 a}(\%)^{b}$ & $\mathbf{4 a}(\%)^{b}$ & $\mathbf{5 a}(\%)^{b}$ \\
\hline 1 & $\mathbf{2 a}$ & 26 & 1 & 4 \\
2 & $\mathbf{2 a b}$ & 1 & 2 & nd \\
3 & $\mathbf{2 a c}$ & 1 & 2 & nd \\
4 & $\mathbf{2 a d}$ & 2 & 3 & nd \\
\hline
\end{tabular}

${ }^{a}$ Reaction conditions (unless otherwise specified): $1 \mathbf{a}(0.3 \mathrm{mmol}, 1.0$ equiv), 2a (2.0 equiv). The yield was determined by ${ }^{19} \mathrm{~F}$ NMR using fluorobenzene as an internal standard. ${ }^{b}$ The yield was calculated based on 1a. nd, not detected. 
Table S3. Screening of the solvents ${ }^{a}$

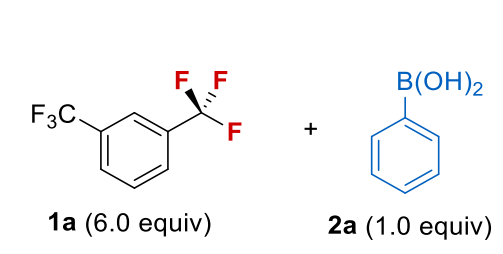

$\mathrm{Pd}\left({ }^{\mathrm{t}} \mathrm{Bu}_{2} \mathrm{PhP}\right)_{2} \mathrm{Cl}_{2}(5 \mathrm{~mol} \%)$ Xantphos (10 mol \%) $\mathrm{K}_{2} \mathrm{CO}_{3}$ (2.0 equiv) solvent Blue LED $(12 \mathrm{~W})$ $50{ }^{\circ} \mathrm{C}, 12 \mathrm{~h}$

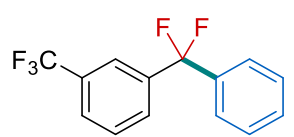<smiles>FC(F)(F)c1cccc(C(F)(F)F)c1</smiles>

$3 a$

$4 a$

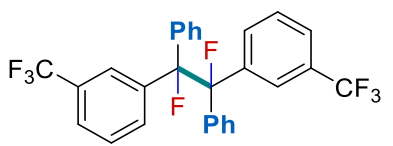

$5 a$

\begin{tabular}{ccccc}
\hline entry & solvent & $\mathbf{3 a}(\%)^{b}$ & $\mathbf{4 a}(\%)^{c}$ & $\mathbf{5 a}(\%)^{b}$ \\
\hline 1 & dioxane & 12 & 1 & 0 \\
2 & DMSO & nd & nd & nd \\
3 & MeCN & 1 & $<1$ & nd \\
4 & DMF & nd & $<1$ & nd \\
5 & DCE & nd & $<1$ & nd \\
$\mathbf{6}$ & THF & $\mathbf{1 9}$ & $\mathbf{2}$ & $\mathbf{9}$ \\
7 & benzene & 8 & $<1$ & nd \\
\hline
\end{tabular}

${ }^{a}$ Reaction conditions (unless otherwise specified): 1a (6.0 equiv), 2a ( $0.3 \mathrm{mmol}, 1.0$ equiv). The yield was determined by ${ }^{19} \mathrm{~F}$ NMR using fluorobenzene as an internal standard. ${ }^{b}$ The yield was calculated based on $\mathbf{2 a} .{ }^{c}$ The yield was calculated based on 1a. nd, not detected. 
Table S4. Screening of the loading amount of Xantphos ${ }^{a}$

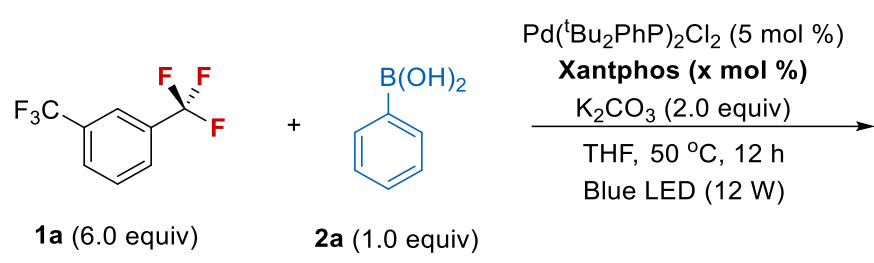

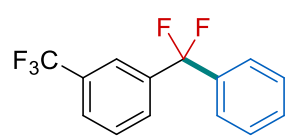

3a

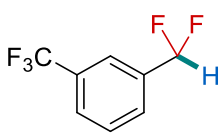

$4 \mathbf{a}$

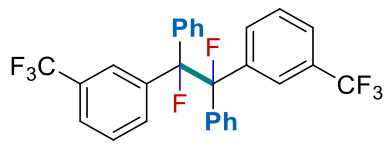

$5 \mathbf{a}$

\begin{tabular}{ccccc}
\hline entry & Xantphos $(\mathrm{x})$ & $\mathbf{3 a}(\%)^{b}$ & $\mathbf{4 a}(\%)^{c}$ & $\mathbf{5 a}(\%)^{b}$ \\
\hline 1 & 6 & 19 & 1 & 5 \\
$\mathbf{2}$ & $\mathbf{8}$ & $\mathbf{2 3}$ & $\mathbf{1}$ & $\mathbf{5}$ \\
3 & 10 & 19 & 2 & 9 \\
4 & 12 & 9 & 2 & 11 \\
\hline
\end{tabular}

${ }^{a}$ Reaction conditions (unless otherwise specified): 1a (6.0 equiv), 2a (0.3 mmol, 1.0 equiv). The yield was determined by ${ }^{19} \mathrm{~F}$ NMR using fluorobenzene as an internal standard. ${ }^{b}$ The yield was calculated based on $\mathbf{2} \mathbf{a} .{ }^{c}$ The yield was calculated based on 1a. nd, not detected. 
Table S5. Screening of the bases $^{a}$

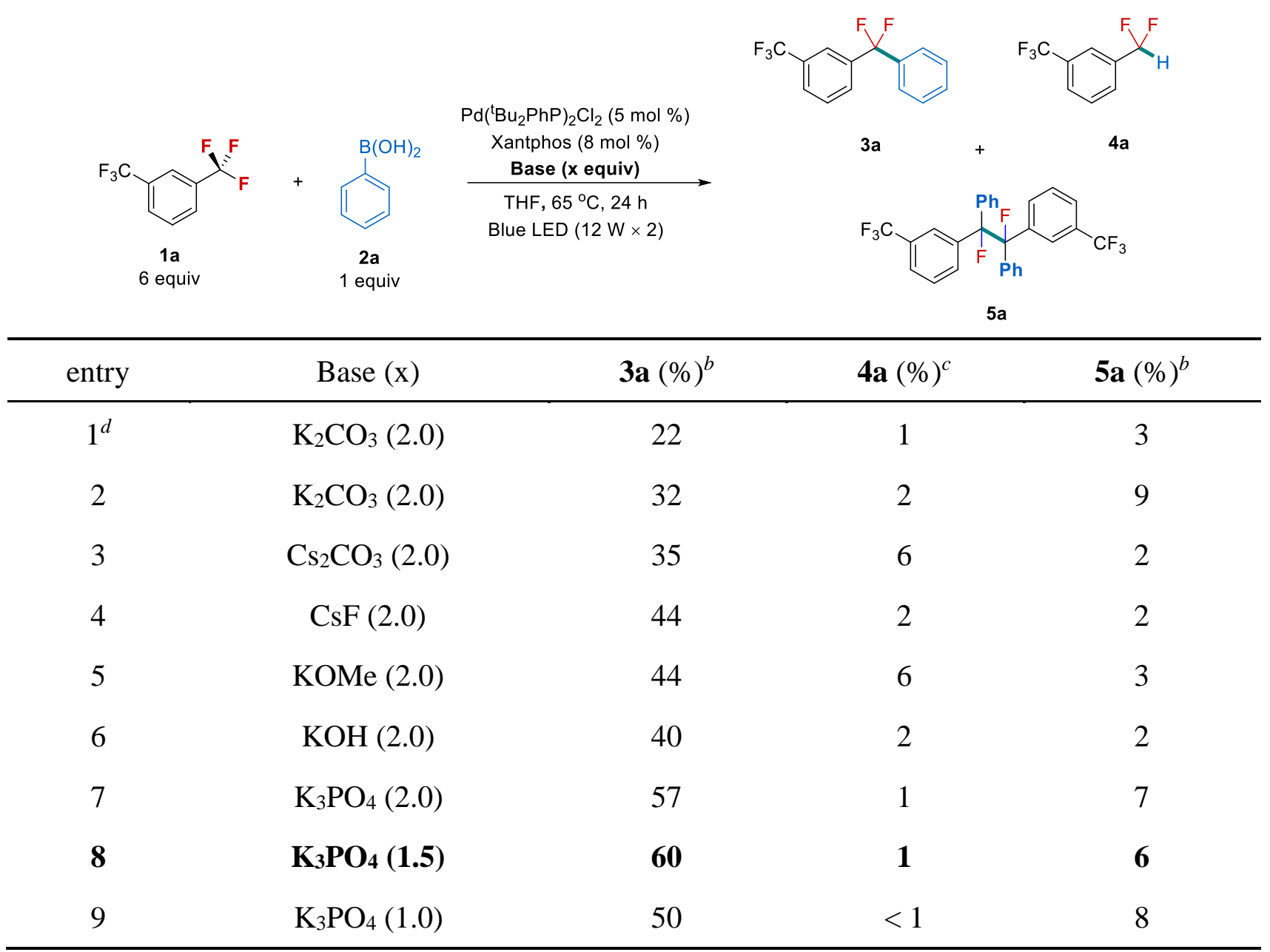

${ }^{a}$ Reaction conditions (unless otherwise specified): 1a (6.0 equiv), 2a (0.3 mmol, 1.0 equiv). The yield was determined by ${ }^{19} \mathrm{~F}$ NMR using fluorobenzene as an internal standard. ${ }^{b}$ The yield was calculated based on 2a. ${ }^{c}$ The yield was calculated based on 1a. ${ }^{d}$ Reaction run at $50{ }^{\circ} \mathrm{C}, 12 \mathrm{~h}, 12 \mathrm{~W}$ blue LED. nd, not detected. 
Table S6. Optimization of the reaction conditions ${ }^{a}$

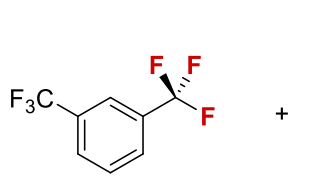

$1 \mathrm{a}$
6 equiv
$\mathrm{Pd}\left({ }^{\mathrm{t} B \mathrm{Bu}_{2}} \mathrm{PhP}\right)_{2} \mathrm{Cl}_{2}(5 \mathrm{~mol} \%)$

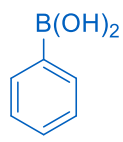

2a 1 equiv
Xantphos (8 mol \%)

$\mathrm{K}_{3} \mathrm{PO}_{4}$ (1.5 equiv)

THF, $65^{\circ} \mathrm{C}, 24 \mathrm{~h}$

Blue LED $(12 \mathrm{~W} \times 2)$<smiles>FC(F)(F)c1cccc(C(F)(F)c2ccccc2)c1</smiles><smiles>FC(F)(F)c1cccc(C(F)(F)F)c1</smiles>

$3 a$

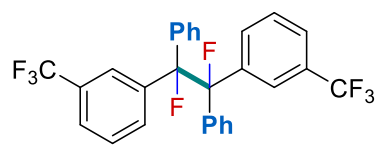

5

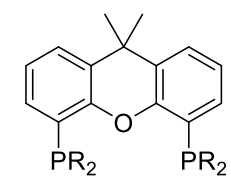

$\mathrm{R}=\mathrm{Ph}$, XantPhos $\mathrm{R}={ }^{t} \mathrm{Bu},{ }^{t} \mathrm{Bu}-$ Xantphos

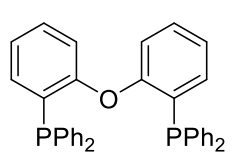

DPEPhos

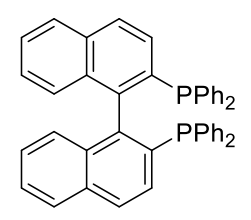

BINAP

\begin{tabular}{|c|c|c|c|c|}
\hline entry & Conditions & $\mathbf{3 a}(\%)^{b}$ & $\mathbf{4 a}(\%)^{c}$ & $\mathbf{5 a}(\%)^{b}$ \\
\hline 1 & "standard conditions" & 60 & 1 & 6 \\
\hline 2 & No $\mathrm{Pd}\left(\mathrm{P}^{t} \mathrm{Bu}_{2} \mathrm{Ph}\right)_{2} \mathrm{Cl}_{2}$ & nd & -- & nd \\
\hline 3 & No Xantphos & nd & -- & nd \\
\hline 4 & No light & nd & -- & nd \\
\hline 5 & $\mathrm{Pd}\left(\mathrm{PPh}_{3}\right)_{4}$ instead of $\mathrm{Pd}\left(\mathrm{P}^{t} \mathrm{Bu}_{2} \mathrm{Ph}\right)_{2} \mathrm{Cl}_{2}$ & 47 & 4 & 2 \\
\hline 6 & $\mathrm{Pd}\left(\mathrm{PPh}_{3}\right)_{4}$ instead of $\mathrm{Pd}\left(\mathrm{P}^{t} \mathrm{Bu}_{2} \mathrm{Ph}\right)_{2} \mathrm{Cl}_{2}$ and Xantphos & 22 & 4 & nd \\
\hline 7 & $\mathrm{Pd}\left(\mathrm{P}^{t} \mathrm{Bu}_{2} \mathrm{Ph}\right)_{2}$ instead of $\mathrm{Pd}\left(\mathrm{P}^{t} \mathrm{Bu}_{2} \mathrm{Ph}\right)_{2} \mathrm{Cl}_{2}$ and Xantphos & nd & -- & nd \\
\hline 8 & $\operatorname{Ir}(\mathrm{ppy})_{3}(1 \mathrm{~mol} \%)$ used as an additional photocatalyst & 58 & 1 & 5 \\
\hline 9 & ${ }^{t} \mathrm{Bu}-\mathrm{X}$ antphos instead of Xantphos & nd & $<1$ & 3 \\
\hline 10 & DPEphos instead of Xantphos & 15 & $<1$ & 3 \\
\hline 11 & BINAP instead of Xantphos & 1 & $<1$ & 1 \\
\hline 12 & $\mathbf{1 a}$ (1 equiv) & 37 & 5 & 7 \\
\hline 13 & $\mathbf{1 a}(2$ equiv $)$ & 51 & 3 & 8 \\
\hline 14 & $\mathbf{1 a}$ (3 equiv) & 57 & 2 & 7 \\
\hline 15 & $\mathbf{1 a}$ (9 equiv) & 61 & 1 & 5 \\
\hline
\end{tabular}

${ }^{a}$ Reaction conditions (unless otherwise specified): 1a ( 6.0 equiv), 2a ( $0.3 \mathrm{mmol}, 1.0$ equiv). The yield was determined by ${ }^{19} \mathrm{~F}$ NMR using fluorobenzene as an internal standard. ${ }^{b}$ The yield was calculated based on 2a. ${ }^{c}$ The yield was calculated based on 1a. nd, not detected. 
Table S7. Optimization of the reaction conditions with 1 equiv of $\mathrm{ArCF}_{3}{ }^{a}$

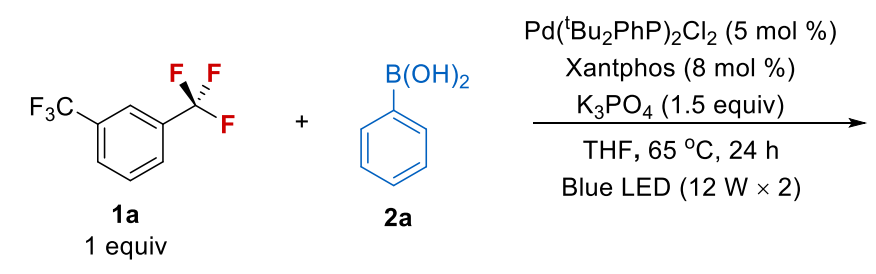

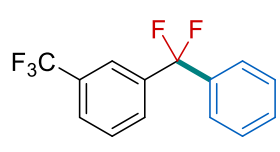

$3 a$

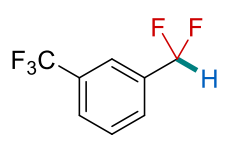

$4 a$

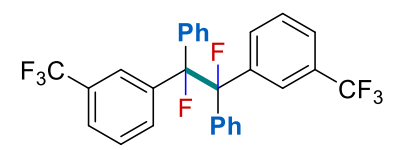

$5 a$

\begin{tabular}{cccccc}
\hline entry & $\mathrm{PhB}(\mathrm{OH})_{2}$ & base & $\mathbf{3 a}(\%)^{b}$ & $\mathbf{4 a}(\%)^{b}$ & $\mathbf{5 a}(\%)^{b}$ \\
\hline 1 & $\mathbf{2 a}(1.0$ equiv) & $\mathrm{K}_{3} \mathrm{PO}_{4}(1.5$ equiv $)$ & 37 & 5 & 7 \\
2 & $\mathbf{2 a}(1.5$ equiv) & $\mathrm{K}_{3} \mathrm{PO}_{4}(2.0$ equiv $)$ & 44 & 7 & 9 \\
3 & $\mathbf{2 a}(2.0$ equiv) & $\mathrm{K}_{3} \mathrm{PO}_{4}(2.5$ equiv $)$ & 47 & 7 & 11 \\
4 & $\mathbf{2 a}(3.0$ equiv) & $\mathrm{K}_{3} \mathrm{PO}_{4}(3.5$ equiv $)$ & 36 & 9 & 16 \\
\hline 5 & $\mathbf{2 a}(2.0$ equiv) & $\mathrm{KOH}(1.5$ equiv) & 48 & 6 & 4 \\
$\mathbf{6}$ & $\mathbf{2 a}(\mathbf{2 . 0}$ equiv) & $\mathbf{K O H}(\mathbf{2 . 0}$ equiv) & $\mathbf{5 2}$ & $\mathbf{5}$ & $\mathbf{5}$ \\
7 & $\mathbf{2 a}(2.0$ equiv $)$ & $\mathrm{KOH}(2.5$ equiv $)$ & 45 & 6 & 4 \\
\hline
\end{tabular}

${ }^{a}$ Reaction conditions (unless otherwise specified): 1a ( $0.3 \mathrm{mmol}, 1.0$ equiv), 2a (1.0-3.0 equiv). The yield was determined by ${ }^{19} \mathrm{~F}$ NMR using fluorobenzene as an internal standard. ${ }^{b}$ The yield was calculated based on 1a. nd, not detected. 
3. Preparation of trifluoromethylarenes<smiles>FC(F)(F)c1cccc(C(F)(F)F)c1</smiles>

1a<smiles>O=C(Nc1cc(C(F)(F)F)cc(C(F)(F)F)c1)OC(=O)OCc1ccccc1</smiles>

10<smiles>FC(F)(F)c1cccnc1</smiles>

$1 \mathrm{t}$<smiles>FC(F)(F)c1ccccc1</smiles>

1y<smiles>FC(F)(F)c1ccccc1C(F)(F)F</smiles>

$1 k$<smiles>FC(F)(F)c1cc(Nc2ccccc2)cc(C(F)(F)F)c1</smiles>

$1 \mathrm{p}$<smiles>O=C(c1cccc(C(F)(F)F)c1)N1CCOCC1</smiles>

1u<smiles>COc1cccc(C(F)(F)F)c1</smiles>

$1 z$<smiles>COc1cc(C(F)(F)F)cc(C(F)(F)F)c1</smiles>

11<smiles>FC(F)(F)c1cc(-c2ccccc2)cc(C(F)(F)F)c1</smiles>

1q<smiles>CCNS(=O)(=O)c1cccc(C(F)(F)F)c1</smiles>

$1 \mathrm{v}$<smiles>COCc1cc(C(F)(F)F)cc(C(F)(F)F)c1</smiles>

$1 \mathrm{~m}$<smiles>FC(F)(F)c1ccc(-c2ccccc2)cc1</smiles>

$1 \mathrm{r}$<smiles>N#Cc1cccc(C(F)(F)F)c1</smiles>

$1 w$<smiles>FC(F)(F)c1cc(COCCCCl)cc(C(F)(F)F)c1</smiles>

$1 n$<smiles>FC(F)(F)c1ccccn1</smiles>

$1 \mathrm{~s}$<smiles>N#Cc1ccc(C(F)(F)F)cc1</smiles>

$1 x$

\section{Structures of trifluoromethylarenes}

Note: Substrates 1a, 1k-l, $1 \mathbf{s - t}$ and $\mathbf{1 w - z}$ are commercially available. Other substrates are prepared from $\mathrm{CF}_{3}$ containing compounds which are commercially available.

\subsection{Preparation of 1-(methoxymethyl)-3,5-bis(trifluoromethyl)benzene $1 \mathrm{~m}$}

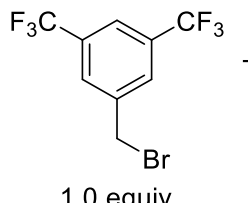

$\mathrm{MeONa}$

$\mathrm{MeOH}(0.33 \mathrm{M})$

$$
\mathrm{rt}, 6 \mathrm{~h}
$$

2.0 equiv

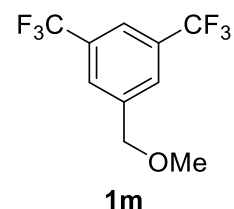

$1 \mathrm{~m}$

To a $100 \mathrm{~mL}$ Schlenk tube was added MeONa (1.08 g, $20 \mathrm{mmol}, 2.0$ equiv), and the tube was evacuated and backfilled with $\mathrm{Ar}$ (3 times). $\mathrm{MeOH}(30 \mathrm{~mL})$ and 1-(bromomethyl)-3,5bis(trifluoromethyl)benzene (1.83 $\mathrm{mL}, 10.0 \mathrm{mmol}, 1.0$ equiv) was added. The reaction mixture was stirrred at $\mathrm{rt}$ for $6 \mathrm{~h}$. Then, the reaction mixture was poured into water $(50 \mathrm{~mL})$. The reaction mixture was extracted with ethyl acetate $(30 \mathrm{~mL} \times 2)$. The combined organic solution was washed by brine, dried over anhydrous sodium sulfate, filtered and concentrated. The product was isolated by flash column chromatography (3\% Ethyl acetate/Petroleum ether) as a colorless oil (1.35 g, 52\% yield). ${ }^{1} \mathbf{H}$ NMR $\left(400 \mathrm{MHz}, \mathrm{CDCl}_{3}\right) \delta 7.78(\mathrm{~s}, 3 \mathrm{H}), 4.55(\mathrm{~s}, 2 \mathrm{H}), 3.45(\mathrm{~s}, 3 \mathrm{H}) .{ }^{\mathbf{1}} \mathbf{F}$ NMR $\left(376 \mathrm{MHz}, \mathrm{CDCl}_{3}\right) \delta-$ 
63.0 (s). ${ }^{13} \mathrm{C}$ NMR $\left(101 \mathrm{MHz}, \mathrm{CDCl}_{3}\right) \delta 141.0,131.7(\mathrm{q}, J=33.4 \mathrm{~Hz}), 127.3-127.1(\mathrm{~m}), 123.3(\mathrm{q}, J$ $=272.6 \mathrm{~Hz}), 121.6-121.3(\mathrm{~m}), 73.1,58.7 . \mathbf{I R}\left(\right.$ thin film) $v_{\max } 2996,2935,1623,1460 \mathrm{~cm}^{-1} . \mathbf{M S}(\mathrm{EI})$ m/z (\%) $258\left(\mathrm{M}^{+}\right), 239,227$ (100), 209, 189, 177. HRMS (EI) calculated for $\mathrm{C}_{10} \mathrm{H}_{8} \mathrm{OF}_{6}:$ 258.0474; Found: $258.0477\left(\mathrm{M}^{+}\right)$.

\subsection{Preparation of 1-((3-chloropropoxy)methyl)-3,5-bis(trifluoromethyl)benzene 1n}
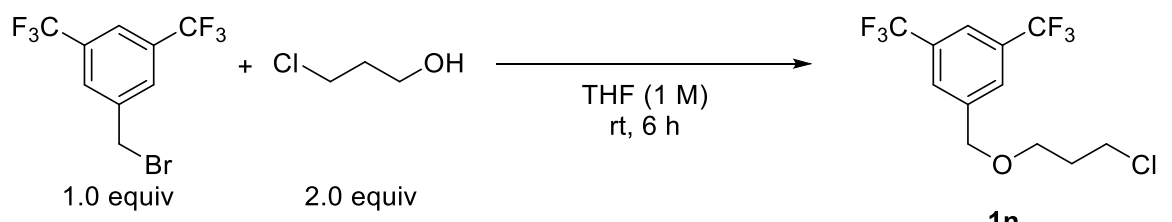

$1 n$

To a stirred solution of t-BuONa (3.03 g, $31.5 \mathrm{mmol}, 1.05$ equiv) in dry THF (20 mL) under nitrogen was added 3-chioropropan-1-ol (2.51 mL, $30 \mathrm{mmol}, 1$ equiv) and $\mathrm{ArCH}_{2} \mathrm{Br}(5.50 \mathrm{~mL}, 30 \mathrm{mmol}, 1$ equiv) and 3-chioropropan-1-ol $\left(2.51 \mathrm{~mL}, 30 \mathrm{mmol}, 1\right.$ equiv) at $0{ }^{\circ} \mathrm{C}$. After addition the mixture was stirred overnight at $\mathrm{rt}$. Then, the reaction mixture was poured into water $(50 \mathrm{~mL})$. The reaction mixture was extracted with ethyl acetate $(30 \mathrm{~mL} \times 2)$. The combined organic solution was washed by brine, dried over anhydrous sodium sulfate, filtered and concentrated. The product was isolated by flash column chromatography (3\% Ethyl acetate/Petroleum ether) as a colorless oil (9.56 g, $99 \%$ yield). ${ }^{1} \mathbf{H}$ NMR (400 MHz, $\left.\mathrm{CDCl}_{3}\right) \delta 7.87-7.72(\mathrm{~m}, 3 \mathrm{H}), 4.63(\mathrm{~s}, 2 \mathrm{H}), 3.81-3.61(\mathrm{~m}, 4 \mathrm{H}), 2.10(\mathrm{tt}, J=6.1$, $6.1 \mathrm{~Hz}, 2 \mathrm{H}) .{ }^{19} \mathbf{F}$ NMR $\left(376 \mathrm{MHz}, \mathrm{CDCl}_{3}\right) \delta-62.9(\mathrm{~s}) .{ }^{13} \mathbf{C ~ N M R}\left(126 \mathrm{MHz}, \mathrm{CDCl}_{3}\right) \delta 141.0,131.7$ (q, $J=33.2 \mathrm{~Hz}), 127.2(\mathrm{q}, J=4.0 \mathrm{~Hz}), 123.3(\mathrm{q}, J=272.7 \mathrm{~Hz}), 121.6-121.4(\mathrm{~m}), 71.6,67.3,41.6,32.5$. IR (thin film) $v_{\max } 3073,2873,1625 \mathrm{~cm}^{-1}$. MS (EI) (\%) m/z $320\left(\mathrm{M}^{+}\right), 243,228$ (100), 209. HRMS (EI) calculated for $\mathrm{C}_{12} \mathrm{H}_{11} \mathrm{ClF}_{6} \mathrm{O}: 320.0403$; Found: $320.0399\left(\mathrm{M}^{+}\right)$.

\subsection{Preparation of tert-butyl (3,5-bis(trifluoromethyl)phenyl)carbamate 10}
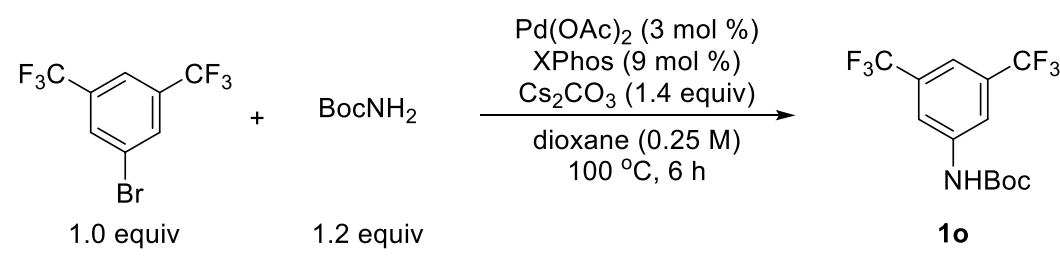

To a $250 \mathrm{~mL}$ Schlenk tube were added $\mathrm{Pd}(\mathrm{OAc})_{2}(67 \mathrm{mg}, 0.3 \mathrm{mmol}, 3 \mathrm{~mol} \%)$, XPhos (43 mg, 0.9 mmol, $9 \mathrm{~mol} \%$ ) and $\mathrm{Cs}_{2} \mathrm{CO}_{3}(4.56 \mathrm{~g}, 14 \mathrm{mmol}, 1.4$ equiv). The reaction mixture was then evacuated 
and backfilled with Ar (3 times). Dioxane (40 mL), BocNH 2 (1.41 g, 12 mmol, 1.2 equiv) and 3,5bis(trifluoromethyl)bromobenzene $(1.72 \mathrm{~mL}, 10 \mathrm{mmol}, 1$ equiv) were added. The reaction mixture was stirring at $100{ }^{\circ} \mathrm{C}$ for $6 \mathrm{~h}$. Upon cooling to rt, the reaction mixture was poured into water $(50 \mathrm{~mL})$ and extracted with ethyl acetate $(30 \mathrm{~mL} \times 2)$. The combined organic solution was washed by brine, dried over anhydrous sodium sulfate, filtered and concentrated. The product was isolated by flash column chromatography (10\% Ethyl acetate:Petroleum ether) as a yellow solid (1.48 g, 45\% yield) (m.p. 135$\left.138{ }^{\circ} \mathrm{C}\right)$. Compound 10 is known. ${ }^{2}{ }^{1} \mathbf{H}$ NMR $\left(400 \mathrm{MHz}, \mathrm{CDCl}_{3}\right) \delta 7.86(\mathrm{~s}, 2 \mathrm{H}), 7.52(\mathrm{~s}, 1 \mathrm{H}), 6.85(\mathrm{~s}$, 1H), 1.53 (s, 9H). ${ }^{19} \mathbf{F}$ NMR $\left(376 \mathrm{MHz}, \mathrm{CDCl}_{3}\right) \delta-63.1$ (s). ${ }^{13} \mathbf{C} \mathbf{N M R}\left(101 \mathrm{MHz}, \mathrm{CDCl}_{3}\right) \delta 152.1$, $139.9,132.3(\mathrm{q}, J=33.5 \mathrm{~Hz}), 123.1(\mathrm{q}, J=272.8 \mathrm{~Hz}), 118.0,116.2(\mathrm{~m}), 82.0,28.2$.

\subsection{Preparation of $N$-methyl- $N$-phenyl-3,5-bis(trifluoromethyl)aniline 1p}
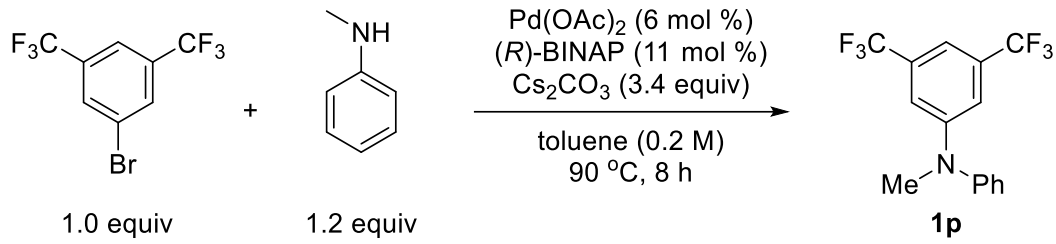

To a $250 \mathrm{~mL}$ Schlenk tube were added $\mathrm{Pd}(\mathrm{OAc})_{2}(135 \mathrm{mg}, 0.6 \mathrm{mmol}, 6 \mathrm{~mol} \%),(R)$-BINAP (685 mg, $1.1 \mathrm{mmol}, 11 \mathrm{~mol} \%)$ and $\mathrm{Cs}_{2} \mathrm{CO}_{3}(11.11 \mathrm{~g}, 34 \mathrm{mmol}, 3.4$ equiv). The reaction mixture was then evacuated and backfilled with Ar (3 times). Toluene $(50 \mathrm{~mL}), N$-methylaniline $(1.30 \mathrm{~mL}, 12 \mathrm{mmol}$, 1.2 equiv) and 3,5-bis(trifluoromethyl)bromobenzene (1.72 mL, $10 \mathrm{mmol}, 1$ equiv) were added. The reaction mixture was stirred at $90{ }^{\circ} \mathrm{C}$ for $8 \mathrm{~h}$. Upon cooling to rt, the reaction mixture was poured into water $(50 \mathrm{~mL})$ and extracted with ethyl acetate $(30 \mathrm{~mL} \times 2)$. The combined organic solution was washed by brine, dried over anhydrous sodium sulfate, filtered and concentrated. The product was isolated by flash column chromatography (10\% Ethyl acetate/Petroleum ether) as a colorless oil (3.12 g, $98 \%$ yield). Compound 1p is known. ${ }^{1}{ }^{1} \mathbf{H}$ NMR $\left(400 \mathrm{MHz}, \mathrm{CDCl}_{3}\right) \delta 7.42(\mathrm{dd}, J=7.7,7.7 \mathrm{~Hz}, 2 \mathrm{H}), 7.24$ $(\mathrm{dd}, J=7.7,7.7 \mathrm{~Hz}, 1 \mathrm{H}), 7.23(\mathrm{~s}, 1 \mathrm{H}), 7.19(\mathrm{~d}, J=7.7 \mathrm{~Hz}, 2 \mathrm{H}), 7.14(\mathrm{~s}, 2 \mathrm{H}), 3.37(\mathrm{~s}, 3 \mathrm{H}) .{ }^{19} \mathbf{F} \mathbf{N M R}$ $\left(376 \mathrm{MHz}, \mathrm{CDCl}_{3}\right) \delta-63.2(\mathrm{~s}) .{ }^{13} \mathbf{C} \mathbf{N M R}\left(101 \mathrm{MHz}, \mathrm{CDCl}_{3}\right) \delta 149.8,147.0,132.2(\mathrm{q}, J=32.7 \mathrm{~Hz})$, 130.2, 125.8, 125.5, $123.6(\mathrm{q}, J=272.6 \mathrm{~Hz}), 114.3-114.1(\mathrm{~m}), 111.0(\mathrm{~m}), 40.4$. 


\subsection{Preparation of 3,5-bis(trifluoromethyl)-1,1'-biphenyl 1q}
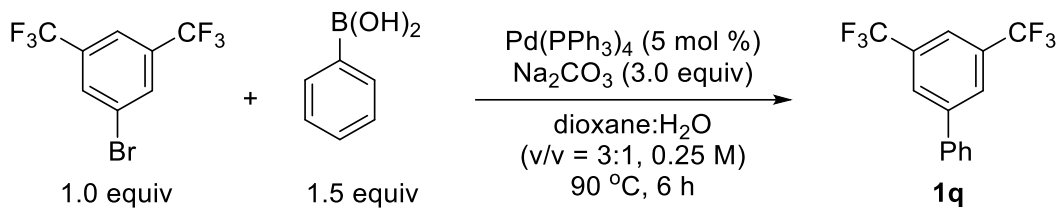

To a $250 \mathrm{~mL}$ Schlenk tube were added $\mathrm{PhB}(\mathrm{OH})_{2}\left(1.83 \mathrm{~g}, 15 \mathrm{mmol}, 1.5\right.$ equiv), $\mathrm{Na}_{2} \mathrm{CO}_{3}(3.18 \mathrm{~g}, 30$ mmol, 3.0 equiv), $\mathrm{Pd}\left(\mathrm{PPh}_{3}\right)_{4}(578 \mathrm{mg}, 0.5 \mathrm{mmol}, 5 \mathrm{~mol} \%)$. The reaction mixture was then evacuated and backfilled with $\mathrm{Ar}$ (3 times). A mixture of solvent (dioxane $/ \mathrm{H}_{2} \mathrm{O}=3: 1,40 \mathrm{~mL}$ ) and 3,5bis(trifluoromethyl)bromobenzene $(1.72 \mathrm{~mL}, 10 \mathrm{mmol}, 1$ equiv) were added. The reaction mixture was stirred at $90{ }^{\circ} \mathrm{C}$ for $6 \mathrm{~h}$. Upon cooling to rt, the reaction mixture was poured into water $(50 \mathrm{~mL})$ and extracted with ethyl acetate $(30 \mathrm{~mL} \times 2)$. The combined organic solution was washed by brine, dried over anhydrous sodium sulfate, filtered and concentrated. The product was isolated by flash column chromatography (petroleum ether) as a colorless oil (2.97 g, 99\% yield). Compound 1q is known. ${ }^{3} \mathbf{H}$ NMR $\left(400 \mathrm{MHz}, \mathrm{CDCl}_{3}\right) \delta 8.03(\mathrm{~s}, 2 \mathrm{H}), 7.88(\mathrm{~s}, 1 \mathrm{H}), 7.62(\mathrm{~d}, J=7.2 \mathrm{~Hz}, 2 \mathrm{H}), 7.52(\mathrm{dd}, J=7.2,7.2$ $\mathrm{Hz}, 2 \mathrm{H}), 7.47(\mathrm{dd}, J=7.2,7.2 \mathrm{~Hz}, 2 \mathrm{H}) .{ }^{\mathbf{1 9}} \mathbf{F} \mathbf{N M R}\left(376 \mathrm{MHz}, \mathrm{CDCl}_{3}\right) \delta-62.9(\mathrm{~s}) .{ }^{13} \mathbf{C ~ N M R}(101 \mathrm{MHz}$, $\left.\mathrm{CDCl}_{3}\right) \delta 143.3,138.2,132.1(\mathrm{q}, J=33.2 \mathrm{~Hz}), 129.3,128.9,127.2,127.3-127.1(\mathrm{~m}), 123.4(\mathrm{q}, J=$ $272.6 \mathrm{~Hz}), 120.9$ (m).

\subsection{Preparation of morpholino(3-(trifluoromethyl)phenyl)methanone 1u}

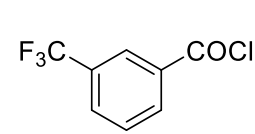

1 equiv<smiles>C1COCCN1</smiles>

3 equiv

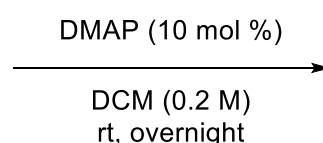

rt, overnight

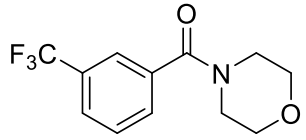

$1 \mathrm{u}$

To a $250 \mathrm{~mL}$ round-bottom flask were added 3-(trifluoromethyl)benzoyl chloride $(1.50 \mathrm{~mL}, 10 \mathrm{mmol}$, 1 equiv), DMAP (0.12 g, $1 \mathrm{mmol}, 0.1$ equiv) and DCM (50 mL). Morpholine (2.60 mL, $30 \mathrm{mmol}, 3.0$ equiv) was then added at $0{ }^{\circ} \mathrm{C}$. The reaction mixture was stirred at $\mathrm{rt}$ overnight. The reaction mixture was poured into water $(50 \mathrm{~mL})$ and extracted with $\mathrm{DCM}(30 \mathrm{~mL} \times 2)$. The combined organic solution was washed by brine, dried over anhydrous sodium sulfate, filtered and concentrated. The product was isolated by flash column chromatography (6\% Ethyl acetate/Petroleum ether) as a white solid (2.35 g, 91\% yield) (m.p. 64-68 $\left.{ }^{\circ} \mathrm{C}\right)$. Compound 1u is known. ${ }^{3} \mathbf{1} \mathbf{H}$ NMR $\left(400 \mathrm{MHz}, \mathrm{CDCl}_{3}\right) \delta 7.72-7.64(\mathrm{~m}$, 2H), $7.61-7.52(\mathrm{~m}, 2 \mathrm{H}), 3.77(\mathrm{br}, 4 \mathrm{H}), 3.65(\mathrm{br}, 2 \mathrm{H}), 3.42(\mathrm{br}, 2 \mathrm{H}) .{ }^{19} \mathbf{F}$ NMR $\left(376 \mathrm{MHz}, \mathrm{CDCl}_{3}\right) \delta-$ 
62.9 (s). ${ }^{13} \mathrm{C}$ NMR $\left(101 \mathrm{MHz}, \mathrm{CDCl}_{3}\right) \delta 168.7,136.1,131.1$ (q, $\left.J=32.9 \mathrm{~Hz}\right), 130.3,129.1,126.6(\mathrm{q}$, $J=3.7 \mathrm{~Hz}), 124.1(\mathrm{q}, J=3.8 \mathrm{~Hz}), 123.6(\mathrm{q}, J=272.5 \mathrm{~Hz}), 48.1,42.6$.

\subsection{Preparation of $N, N$-diethyl-3-(trifluoromethyl)benzenesulfonamide $1 v$}
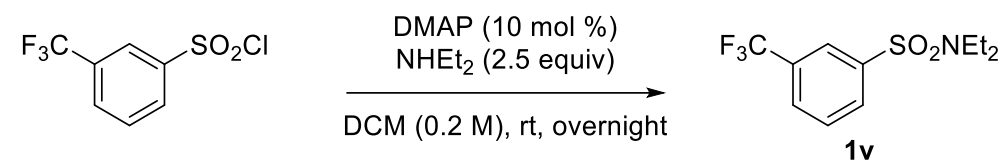

To a $250 \mathrm{~mL}$ round-bottom flask were added $\mathrm{Et}_{2} \mathrm{NH}(2.6 \mathrm{~mL}, 25 \mathrm{mmol}, 2.5$ equiv), DMAP $(0.12 \mathrm{~g}, 1$ mmol, 0.1 equiv), and DCM (50 mL). 3-(Trifluoromethyl)benzenesulfonyl chloride (2.44 g, 10 mmol, 1.0 equiv) was then added at $0{ }^{\circ} \mathrm{C}$. The reaction mixture was stirred at $\mathrm{rt}$ overnight. The reaction mixture was poured into water $(50 \mathrm{~mL})$ and extracted with DCM $(30 \mathrm{~mL} \times 2)$. The combined organic solution was washed by brine, dried over anhydrous sodium sulfate, filtered and concentrated. The product was isolated by flash column chromatography (10\% Ethyl acetate/Petroleum ether) as a colorless oil (2.23 g, 79\% yield). Compound 1v is known. ${ }^{4} \mathbf{H}$ NMR (400 MHz, $\left.\mathrm{CDCl}_{3}\right) \delta 8.06(\mathrm{~s}, 1 \mathrm{H})$, $8.00(\mathrm{~d}, J=7.8 \mathrm{~Hz}, 1 \mathrm{H}), 7.81(\mathrm{~d}, J=7.8 \mathrm{~Hz}, 1 \mathrm{H}), 7.65(\mathrm{dd}, J=7.8,7.8 \mathrm{~Hz}, 1 \mathrm{H}), 3.26(\mathrm{q}, J=7.1 \mathrm{~Hz}$, 4H), $1.14(\mathrm{t}, J=7.1 \mathrm{~Hz}, 6 \mathrm{H}) .{ }^{19} \mathbf{F}$ NMR $\left(376 \mathrm{MHz}, \mathrm{CDCl}_{3}\right) \delta-62.9(\mathrm{~s}) .{ }^{13} \mathbf{C} \mathbf{N M R}\left(101 \mathrm{MHz}, \mathrm{CDCl}_{3}\right)$ $\delta 141.8,131.7(\mathrm{q}, J=33.4 \mathrm{~Hz}), 130.1,129.8,128.8(\mathrm{q}, J=3.5 \mathrm{~Hz}), 123.9(\mathrm{q}, J=3.8 \mathrm{~Hz}), 123.2(\mathrm{q}, J$ $=272.9 \mathrm{~Hz}), 42.1,14.1$. 
4. Visible-light-induced

trifluoromethylarenes 1 with arylboronic acids 2

\subsection{General procedure}

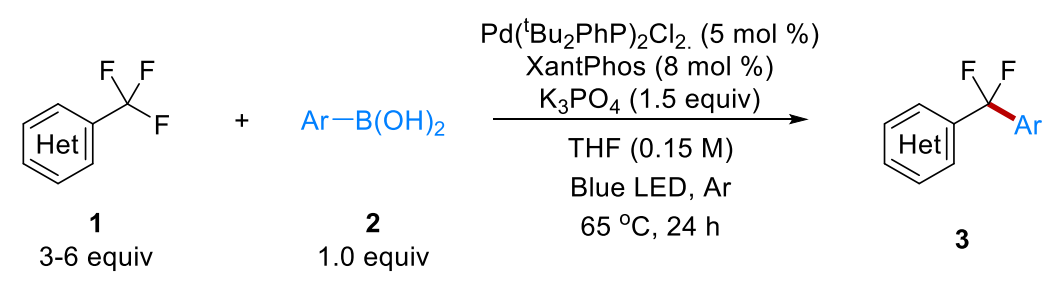

To a $25 \mathrm{~mL}$ of Schlenk tube were added $\mathrm{Pd}\left({ }^{t} \mathrm{Bu}_{2} \mathrm{PhP}\right){ }_{2} \mathrm{Cl}_{2}(9.3 \mathrm{mg}, 0.015 \mathrm{mmol}, 5 \mathrm{~mol} \%)$, Xantphos (13.9 mg, $0.024 \mathrm{mmol}, 8 \mathrm{~mol} \%)$, and $\mathrm{ArB}(\mathrm{OH})_{2}\left(0.3 \mathrm{mmol}, 1.0\right.$ equiv) in the air. $\mathrm{K}_{3} \mathrm{PO}_{4}(95.5 \mathrm{mg}$, 0.45 mmol, 1.5 equiv) was added in the glovebox. The reaction mixture was then evacuated and backfilled with $\mathrm{Ar}$ (3 times). $\mathrm{ArCF}_{3}(1.8 \mathrm{mmol}, 6$ equiv) and THF (2.0 mL) were added. After prestirring at $65^{\circ} \mathrm{C}$ for $20 \mathrm{~min}$, the reaction mixture was irradiated under blue LED strips $(12 \mathrm{~W} \times 2)$ for $24 \mathrm{~h}$ at $65{ }^{\circ} \mathrm{C}$ with stirring. The reaction mixture was then cooled to room temperature and the yield was determined by ${ }^{19} \mathrm{~F}$ NMR using fluorobenzene as an internal standard. The resulting mixture was filtered with a pad of celite. The filtrate was concentrated and the residue was purified with flash chromatography or preparative HPLC to afford the product.

\subsection{Characterization Data for Compounds 3 and 5a}

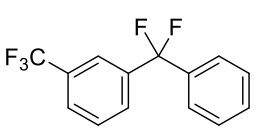

1-(Difluoro(phenyl)methyl)-3-(trifluoromethyl)benzene (3a). The product $(60 \%$

yield determined by ${ }^{19} \mathrm{~F}$ NMR; $31.9 \mathrm{mg}, 39 \%$ yield. Note: The low isolated yield of 3a is because of its volatility) was purified with reverse-phase preparative $\mathrm{HPLC}\left(\mathrm{CH}_{3} \mathrm{CN}: \mathrm{H}_{2} \mathrm{O}=7: 3\right)$ as a pale yellow oil. ${ }^{1} \mathbf{H}$ NMR $\left(400 \mathrm{MHz}, \mathrm{CDCl}_{3}\right) \delta 7.80(\mathrm{~s}, 1 \mathrm{H}), 7.69(\mathrm{dd}, J=7.2,7.2 \mathrm{~Hz}, 2 \mathrm{H}), 7.55$ $(\mathrm{dd}, J=7.8,7.8 \mathrm{~Hz}, 1 \mathrm{H}), 7.52-7.47(\mathrm{~m}, 2 \mathrm{H}), 7.47-7.41(\mathrm{~m}, 3 \mathrm{H}) .{ }^{19} \mathbf{F} \mathbf{N M R}\left(376 \mathrm{MHz}, \mathrm{CDCl}_{3}\right) \delta-$ $62.8(\mathrm{~s}, 3 \mathrm{~F}),-89.3(\mathrm{~s}, 2 \mathrm{~F}) .{ }^{13} \mathrm{C}$ NMR $\left(126 \mathrm{MHz}, \mathrm{CDCl}_{3}\right) \delta 138.7(\mathrm{t}, J=29.3 \mathrm{~Hz}), 136.8(\mathrm{t}, J=28.0$ $\mathrm{Hz}), 131.0(\mathrm{q}, J=33.1 \mathrm{~Hz}), 130.3,129.3(\mathrm{t}, J=5.3 \mathrm{~Hz}), 129.1,128.6,126.9-126.6(\mathrm{~m}), 125.7(\mathrm{t}, J=$ $5.6 \mathrm{~Hz}), 122.8-122.6(\mathrm{~m}), 123.7(\mathrm{q}, J=272.9 \mathrm{~Hz}), 120.0(\mathrm{t}, J=243.0 \mathrm{~Hz}) . \mathbf{I R}\left(\right.$ thin film) $v_{\max } 3070$, 1620, $1496 \mathrm{~cm}^{-1}$. MS (EI) m/z (\%) $272\left(\mathrm{M}^{+}\right), 195,127,77$. HRMS (EI) calculated for $\mathrm{C}_{14} \mathrm{H}_{9} \mathrm{~F}_{5}$ : 272.0624; Found: $272.0616\left(\mathrm{M}^{+}\right)$. 


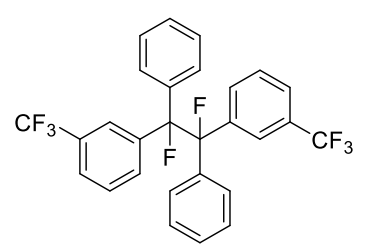

The product ( $6 \%$ yield determined by ${ }^{19} \mathrm{~F}$ NMR) was purified with reversephase preparative HPLC $\left(\mathrm{CH}_{3} \mathrm{CN}: \mathrm{H}_{2} \mathrm{O}=7: 3\right)$ as a colorless oil. (Note: The products $5 \mathbf{a}$ are a mixture of diastereomers $\mathbf{5 a A}$ and $\mathbf{5 a B} . \mathbf{5 a A}: \mathbf{5 a B}=52: 48$.)

${ }^{1} \mathbf{H}$ NMR $\left(400 \mathrm{MHz}, \mathrm{CDCl}_{3}\right) \delta 7.51-7.45(\mathrm{~m}, 4 \mathrm{H}), 7.39-7.29(\mathrm{~m}, 6 \mathrm{H}), 7.26-7.20(\mathrm{~m}, 8 \mathrm{H}) .{ }^{19} \mathrm{~F}$ NMR (376 MHz, $\left.\mathrm{CDCl}_{3}\right) \delta-67.5(\mathrm{~s}, 3 \mathrm{~F}, \mathbf{5 a B}),-67.7(\mathrm{~s}, 3 \mathrm{~F}, \mathbf{5 a A}),-153.2(\mathrm{~s}, 1 \mathrm{~F}, \mathbf{5 a A}),-153.3(\mathrm{~s}, 1 \mathrm{~F}$, 5aB). IR (thin film) $v_{\max } 3066,2930,1601,1496 \mathrm{~cm}^{-1}$. MS (EI) m/z (\%) $487(\mathrm{M}-\mathrm{F})^{+}, 320,253\left(1 / 2 \mathrm{M}^{+}\right.$, 100), 233, 183. HRMS (EI) calculated for $\mathrm{C}_{28} \mathrm{H}_{18} \mathrm{~F}_{8}: 506.1281$; Found: $506.1268\left(\mathrm{M}^{+}\right)$.

GC-MS (EI) spectra of $\mathbf{5 a}$ (two peaks for $\mathbf{5 a A}$ and $\mathbf{5 a B}$ ):
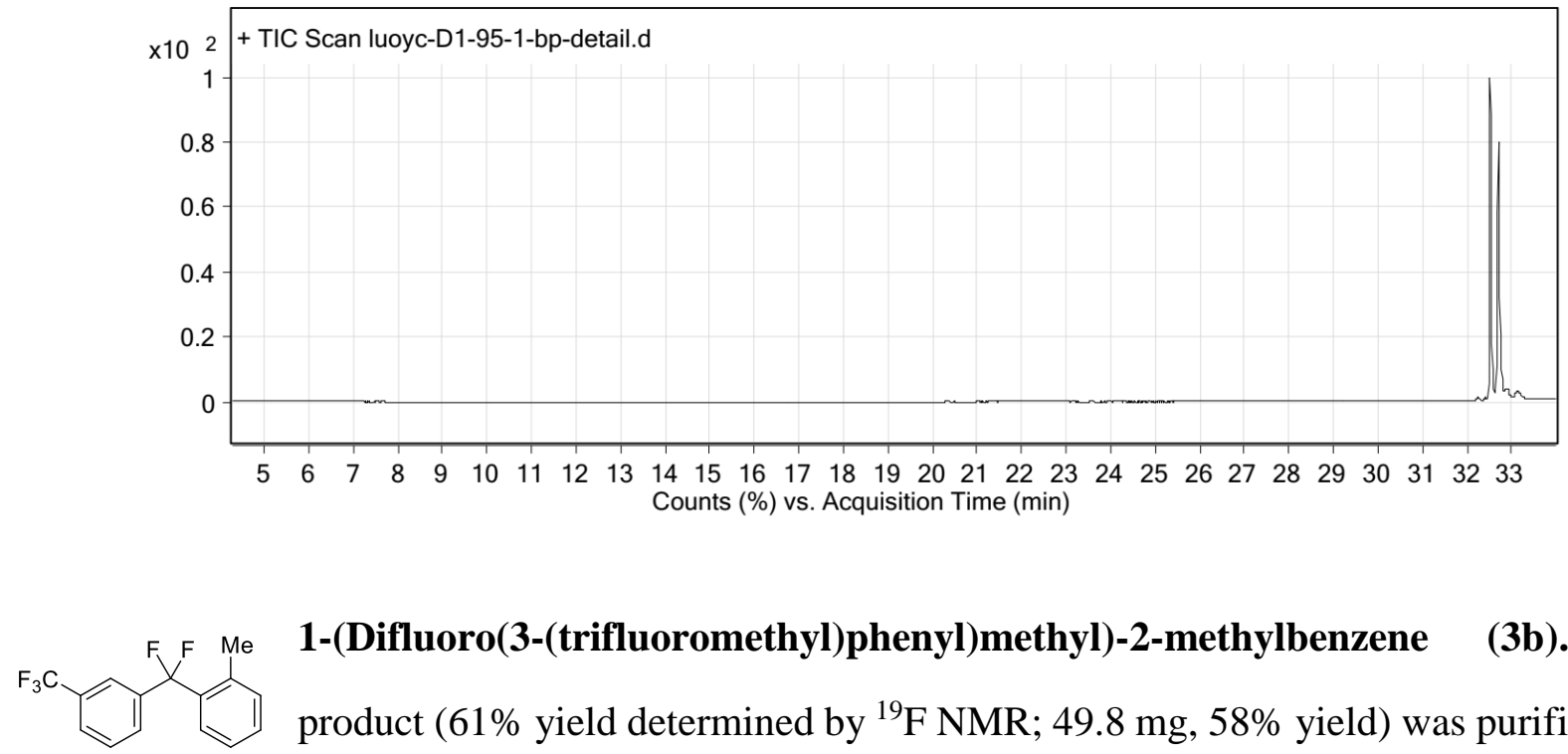

1-(Difluoro(3-(trifluoromethyl)phenyl)methyl)-2-methylbenzene $\quad$ (3b). The product (61\% yield determined by ${ }^{19} \mathrm{~F}$ NMR; $49.8 \mathrm{mg}$, 58\% yield) was purified with reverse-phase preparative HPLC $\left(\mathrm{CH}_{3} \mathrm{CN}: \mathrm{H}_{2} \mathrm{O}=9: 1\right)$ as a pale yellow oil. ${ }^{1} \mathbf{H} \mathbf{N M R}\left(400 \mathrm{MHz}, \mathrm{CDCl}_{3}\right)$ $\delta 7.76(\mathrm{~s}, 1 \mathrm{H}), 7.71(\mathrm{~d}, J=7.7 \mathrm{~Hz}, 1 \mathrm{H}), 7.61(\mathrm{~d}, J=7.9 \mathrm{~Hz}, 1 \mathrm{H}), 7.58-7.51(\mathrm{~m}, 2 \mathrm{H}), 7.39(\mathrm{t}, J=7.4$ Hz, 1H), $7.30(\mathrm{dd}, J=7.7,7.7 \mathrm{~Hz}, 1 \mathrm{H}), 7.23(\mathrm{~d}, J=7.7 \mathrm{~Hz}, 1 \mathrm{H}), 2.21(\mathrm{~s}, 3 \mathrm{H}) .{ }^{19} \mathbf{F}$ NMR $(376 \mathrm{MHz}$, $\left.\mathrm{CDCl}_{3}\right) \delta-62.8(\mathrm{~s}, 3 \mathrm{~F}),-87.1(\mathrm{~s}, 2 \mathrm{~F}) .{ }^{13} \mathrm{C} \mathrm{NMR}\left(126 \mathrm{MHz}, \mathrm{CDCl}_{3}\right) \delta 138.4(\mathrm{t}, J=28.7 \mathrm{~Hz}), 136.4(\mathrm{t}$, $J=2.8 \mathrm{~Hz}), 134.1(\mathrm{t}, J=25.4 \mathrm{~Hz}), 132.0,131.0(\mathrm{q}, J=32.7 \mathrm{~Hz}), 130.5,129.6(\mathrm{t}, J=5.1 \mathrm{~Hz}), 129.1$, $126.9-126.7(\mathrm{~m}), 126.4(\mathrm{t}, J=8.3 \mathrm{~Hz}), 125.8,123.7(\mathrm{q}, J=273.0 \mathrm{~Hz}), 123.1-122.9(\mathrm{~m}), 120.6(\mathrm{t}, J$ $=242.7 \mathrm{~Hz}), 20.2 . \mathbf{I R}\left(\right.$ thin film) $v_{\max } 3075,2958,1619,1490 \mathrm{~cm}^{-1} . \mathbf{M S}(\mathrm{EI}) \mathrm{m} / \mathrm{z}(\%) 286\left(\mathrm{M}^{+}, 100\right)$, 235, 197, 140, 91. HRMS (EI) calculated for $\mathrm{C}_{15} \mathrm{H}_{11} \mathrm{~F}_{5}$ : 286.0781; Found: $286.0774\left(\mathrm{M}^{+}\right)$. 


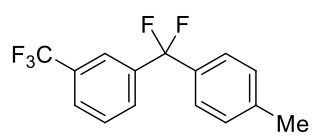

1-(Difluoro(p-tolyl)methyl)-3-(trifluoromethyl)benzene (3c). The product (64\% yield determined by ${ }^{19} \mathrm{~F}$ NMR; $49.0 \mathrm{mg}$, 57\% yield) was purified with reversephase preparative $\mathrm{HPLC}\left(\mathrm{CH}_{3} \mathrm{CN}: \mathrm{H}_{2} \mathrm{O}=8: 2\right)$ as a pale yellow oil. ${ }^{1} \mathbf{H}$ NMR $\left(400 \mathrm{MHz}, \mathrm{CDCl}_{3}\right) \delta 7.79$ (s, 1H), $7.72-7.66(\mathrm{~m}, 2 \mathrm{H}), 7.55(\mathrm{dd}, J=7.8,7.8 \mathrm{~Hz}, 1 \mathrm{H}), 7.38(\mathrm{~d}, J=7.8 \mathrm{~Hz}, 2 \mathrm{H}), 7.24(\mathrm{~d}, J=8.0$ $\mathrm{Hz}, 2 \mathrm{H}), 2.39$ (s, 3H). ${ }^{19} \mathbf{F}$ NMR $\left(376 \mathrm{MHz}, \mathrm{CDCl}_{3}\right) \delta-62.8(\mathrm{~s}, 3 \mathrm{~F}),-88.6$ (s, 2F). ${ }^{13} \mathbf{C}$ NMR $(126 \mathrm{MHz}$, $\left.\mathrm{CDCl}_{3}\right) \delta 140.4(\mathrm{t}, J=1.7 \mathrm{~Hz}), 138.9(\mathrm{t}, J=29.4 \mathrm{~Hz}), 133.9(\mathrm{t}, J=28.1 \mathrm{~Hz}), 131.0(\mathrm{q}, J=32.8 \mathrm{~Hz})$, $129.3(\mathrm{t}, J=4.8 \mathrm{~Hz}), 129.2,129.0,126.7-126.6(\mathrm{~m}), 125.6(\mathrm{t}, J=5.5 \mathrm{~Hz}), 123.7(\mathrm{q}, J=273.0 \mathrm{~Hz})$, $122.8-122.6(\mathrm{~m}), 120.1(\mathrm{t}, J=242.1 \mathrm{~Hz}), 21.3 . \mathbf{I R}\left(\right.$ thin film) $v_{\max } 3038,2927,1619,1516 \mathrm{~cm}^{-1} . \mathbf{M S}$ (EI) m/z (\%) $286\left(\mathrm{M}^{+}\right), 217,195,141$ (100). HRMS (EI) calculated for: $\mathrm{C}_{15} \mathrm{H}_{11} \mathrm{~F}_{5}$ : 286.0781; Found: $286.0771\left(\mathrm{M}^{+}\right)$.

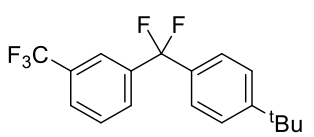

\section{1-((4-(tert-Butyl)phenyl)difluoromethyl)-3-(trifluoromethyl)benzene}

(3d).

The product (63\% yield determined by ${ }^{19} \mathrm{~F}$ NMR; $47.2 \mathrm{mg}, 47 \%$ yield) was purified with reverse-phase preparative $\mathrm{HPLC}\left(\mathrm{CH}_{3} \mathrm{CN}: \mathrm{H}_{2} \mathrm{O}=7: 3\right)$ as a colorless oil. ${ }^{\mathbf{1}} \mathbf{H}$ NMR (400 $\left.\mathrm{MHz}_{\mathrm{CDCl}}\right)^{1}{ }^{1} \mathrm{H} \mathrm{NMR}(400 \mathrm{MHz}$, Chloroform- $d$ ) $\delta 7.83(\mathrm{~s}, 1 \mathrm{H}), 7.70(\mathrm{~d}, J=7.8 \mathrm{~Hz}, 2 \mathrm{H}), 7.55$ (dd, $J$ $=7.8,7.8 \mathrm{~Hz}, 1 \mathrm{H}), 7.46(\mathrm{~d}, J=8.3 \mathrm{~Hz}, 2 \mathrm{H}), 7.42(\mathrm{~d}, J=8.3 \mathrm{~Hz}, 2 \mathrm{H}), 1.34(\mathrm{~s}, 9 \mathrm{H}) .{ }^{19} \mathbf{F} \mathbf{N M R}(376$ $\left.\mathrm{MHz}, \mathrm{CDCl}_{3}\right) \delta-62.7(\mathrm{~s}, 3 \mathrm{~F}),-88.6(\mathrm{~s}, 2 \mathrm{~F}) .{ }^{13} \mathrm{C} \mathrm{NMR}\left(101 \mathrm{MHz}, \mathrm{CDCl}_{3}\right) \delta 153.5,138.9(\mathrm{t}, J=29.5$ Hz), 133.9 (t, $J=28.2 \mathrm{~Hz}), 131.0(\mathrm{q}, J=32.7 \mathrm{~Hz}), 129.3(\mathrm{t}, J=5.1 \mathrm{~Hz}), 129.0,126.7-126.5(\mathrm{~m})$, 125.6, $125.5(\mathrm{t}, J=5.1 \mathrm{~Hz}), 123.7(\mathrm{q}, J=273.8 \mathrm{~Hz}), 122.9-122.6(\mathrm{~m}), 120.15(\mathrm{t}, J=233.4 \mathrm{~Hz}), 34.8$, 31.2. IR (thin film) $v_{\max } 3070,2967,1615,1365 \mathrm{~cm}^{-1}$. MS (EI) m/z (\%) $328\left(\mathrm{M}^{+}\right), 323$ (100), 285, 235, 195, 145. HRMS (EI) calculated for $\mathrm{C}_{18} \mathrm{H}_{17} \mathrm{~F}_{5}: 328.1250$; Found: $328.1239\left(\mathrm{M}^{+}\right)$.

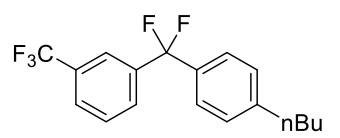

1-((4-Butylphenyl)difluoromethyl)-3-(trifluoromethyl)benzene $\quad(3 e)$. The product (63\% yield determined by ${ }^{19} \mathrm{~F}$ NMR; $52.2 \mathrm{mg}, 53 \%$ yield) was purified with reverse-phase preparative $\mathrm{HPLC}\left(\mathrm{CH}_{3} \mathrm{CN}: \mathrm{H}_{2} \mathrm{O}=8: 2\right)$ as a pale yellow oil. ${ }^{1} \mathbf{H}$ NMR $(400 \mathrm{MHz}$, $\left.\mathrm{CDCl}_{3}\right) \delta 7.82(\mathrm{~s}, 1 \mathrm{H}), 7.70(\mathrm{~d}, J=7.8 \mathrm{~Hz}, 2 \mathrm{H}), 7.55(\mathrm{dd}, J=7.8,7.8 \mathrm{~Hz}, 1 \mathrm{H}), 7.40(\mathrm{~d}, J=7.8 \mathrm{~Hz}$, 2H), $7.25(\mathrm{~d}, J=7.8 \mathrm{~Hz}, 2 \mathrm{H}), 2.65(\mathrm{t}, J=7.8 \mathrm{~Hz}, 2 \mathrm{H}), 1.66-1.56(\mathrm{~m}, 2 \mathrm{H}), 1.42-1.31(\mathrm{~m}, 2 \mathrm{H}), 0.94$ $(\mathrm{t}, J=7.3 \mathrm{~Hz}, 3 \mathrm{H}) .{ }^{19} \mathbf{F}$ NMR $\left(376 \mathrm{MHz}, \mathrm{CDCl}_{3}\right) \delta-62.7$ (s, 3F), -88.5 (s, 2F). ${ }^{13} \mathbf{C}$ NMR $(126 \mathrm{MHz}$, 
$\left.\mathrm{CDCl}_{3}\right) \delta 145.4,138.9(\mathrm{t}, J=29.4 \mathrm{~Hz}), 134.1(\mathrm{t}, J=28.2 \mathrm{~Hz}), 131.0(\mathrm{q}, J=32.8 \mathrm{~Hz}), 129.3(\mathrm{t}, J=4.9$ $\mathrm{Hz}), 129.0,128.6,126.7-126.6(\mathrm{~m}), 125.6(\mathrm{t}, J=5.5 \mathrm{~Hz}), 123.7(\mathrm{q}, J=272.9 \mathrm{~Hz}), 122.8-122.6(\mathrm{~m})$, $120.2(\mathrm{t}, J=242.1 \mathrm{~Hz}), 35.4,33.4,22.3,13.9$. IR (thin film) $v_{\max } 3036,2959,2861,1618,1492 \mathrm{~cm}^{-1}$. MS (EI) m/z (\%) $328\left(\mathrm{M}^{+}\right), 285$ (100), 235, 195, 140. HRMS (EI) calculated for $\mathrm{C}_{18} \mathrm{H}_{17} \mathrm{~F}_{5}: 328.1250$; Found: $328.1243\left(\mathrm{M}^{+}\right)$.

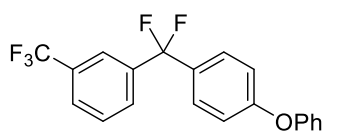

1-(Difluoro(4-phenoxyphenyl)methyl)-3-(trifluoromethyl)benzene (3f). The product (53\% yield determined by ${ }^{19} \mathrm{~F}$ NMR; $50.0 \mathrm{mg}, 46 \%$ yield) was purified with reverse-phase preparative $\mathrm{HPLC}\left(\mathrm{CH}_{3} \mathrm{CN}: \mathrm{H}_{2} \mathrm{O}=8: 2\right)$ as a yellow oil. ${ }^{\mathbf{1}} \mathbf{H} \mathbf{N M R}\left(400 \mathrm{MHz}, \mathrm{CDCl}_{3}\right)$ $\delta 7.82(\mathrm{~s}, 1 \mathrm{H}), 7.75-7.68(\mathrm{~m}, 2 \mathrm{H}), 7.57(\mathrm{dd}, J=7.8,7.8 \mathrm{~Hz}, 1 \mathrm{H}), 7.45(\mathrm{~d}, J=8.4 \mathrm{~Hz}, 2 \mathrm{H}), 7.39$ (dd, $J=7.8,7.8 \mathrm{~Hz}, 2 \mathrm{H}), 7.18(\mathrm{dd}, J=7.4,7.4 \mathrm{~Hz}, 1 \mathrm{H}), 7.06(\mathrm{~d}, J=8.0 \mathrm{~Hz}, 2 \mathrm{H}), 7.03(\mathrm{~d}, J=8.4 \mathrm{~Hz}, 2 \mathrm{H})$. ${ }^{19}$ F NMR $\left(376 \mathrm{MHz}, \mathrm{CDCl}_{3}\right) \delta-62.7(\mathrm{~s}, 3 \mathrm{~F}),-87.7(\mathrm{~s}, 2 \mathrm{~F}) .{ }^{13} \mathbf{C} \mathbf{N M R}\left(126 \mathrm{MHz}, \mathrm{CDCl}_{3}\right) \delta 159.3$, 156.0, $138.7(\mathrm{t}, J=29.4 \mathrm{~Hz}), 131.2(\mathrm{t}, J=28.6 \mathrm{~Hz}), 131.1(\mathrm{q}, J=33.0 \mathrm{~Hz}), 130.0,129.3(\mathrm{t}, J=5.3$ Hz), 129.1, 127.5 (t, $J=5.5 \mathrm{~Hz}), 126.9$ - $126.7(\mathrm{~m}), 123.7(\mathrm{q}, J=273.0 \mathrm{~Hz}), 124.2,122.8$ - $122.6(\mathrm{~m})$, $120.0(\mathrm{t}, J=242.7 \mathrm{~Hz}), 119.7,118.0$. IR (thin film) $v_{\max } 3074,1615,1509,1132 \mathrm{~cm}^{-1} . \mathbf{M S}(\mathrm{EI}) \mathrm{m} / \mathrm{z}$ (\%) $364\left(\mathrm{M}^{+}, 100\right), 272,219,195,141$. HRMS (EI) calculated for $\mathrm{C}_{20} \mathrm{H}_{13} \mathrm{~F}_{5} \mathrm{O}$ : 364.0887; Found: $364.0875\left(\mathrm{M}^{+}\right)$.

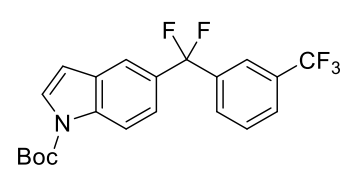

\section{tert-Butyl 5-(difluoro(3-(trifluoromethyl)phenyl)methyl)-1H-indole-1-}

carboxylate (3g). The product (36\% yield determined by ${ }^{19} \mathrm{~F}$ NMR; $25.5 \mathrm{mg}$, $27 \%$ yield) was purified with reverse-phase flash column chromatography $\left(\mathrm{CH}_{3} \mathrm{CN}: \mathrm{H}_{2} \mathrm{O}=7: 3\right)$ as a pale yellow oil. ${ }^{1} \mathrm{H}$ NMR $\left(400 \mathrm{MHz}, \mathrm{CDCl}_{3}\right) \delta 8.20(\mathrm{~d}, J=8.7 \mathrm{~Hz}, 1 \mathrm{H})$, $7.82(\mathrm{~s}, 1 \mathrm{H}), 7.75-7.63(\mathrm{~m}, 4 \mathrm{H}), 7.55(\mathrm{dd}, J=7.9,7.9 \mathrm{~Hz}, 1 \mathrm{H}), 7.42(\mathrm{~d}, J=8.5 \mathrm{~Hz}, 1 \mathrm{H}), 6.61(\mathrm{~d}$, $J=3.8 \mathrm{~Hz}, 1 \mathrm{H}), 1.67(\mathrm{~s}, 9 \mathrm{H}) .{ }^{19} \mathbf{F}$ NMR $\left(376 \mathrm{MHz}, \mathrm{CDCl}_{3}\right) \delta-64.6(\mathrm{~s}, 3 \mathrm{~F}),-89.2(\mathrm{~s}, 2 \mathrm{~F}) .{ }^{13} \mathbf{C ~ N M R}$ $\left(126 \mathrm{MHz}, \mathrm{CDCl}_{3}\right) \delta 149.4,139.2(\mathrm{t}, J=29.6 \mathrm{~Hz}), 135.9,131.2(\mathrm{t}, J=27.9 \mathrm{~Hz}), 130.9(\mathrm{q}, J=32.9$ $\mathrm{Hz}), 130.3,129.4(\mathrm{t}, J=4.6 \mathrm{~Hz}), 129.0,127.24,126.7-126.5(\mathrm{~m}), 123.7(\mathrm{q}, J=272.5 \mathrm{~Hz}), 122.9$ - $122.7(\mathrm{~m}), 121.8(\mathrm{t}, J=5.2 \mathrm{~Hz}), 120.5(\mathrm{t}, J=241.9 \mathrm{~Hz}), 118.6(\mathrm{t}, J=6.0 \mathrm{~Hz}), 115.3,107.4,84.3$, 28.1. IR (thin film) $v_{\max } 2981,1740,1477 \mathrm{~cm}^{-1}$. MS (ESI) \% $412(\mathrm{M}+\mathrm{H})^{+}$. HRMS (ESI) calculated 
for $\mathrm{C}_{21} \mathrm{H}_{18} \mathrm{~F}_{5} \mathrm{NO}_{2}$ : 411.1258; Found: $311.0730(\mathrm{M}-\mathrm{Boc}+\mathrm{H})^{+}$.

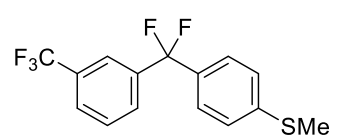

(4-(Difluoro(3-(trifluoromethyl)phenyl)methyl)phenyl)(methyl)sulfane (3h).

The product (49\% yield determined by ${ }^{19} \mathrm{~F}$ NMR; $37.0 \mathrm{mg}$, 39\% yield) was purified with reverse-phase preparative $\mathrm{HPLC}\left(\mathrm{CH}_{3} \mathrm{CN}: \mathrm{H}_{2} \mathrm{O}=7: 3\right)$ as a pale yellow oil. ${ }^{1} \mathbf{H}$ NMR (400 $\left.\mathrm{MHz}, \mathrm{CDCl}_{3}\right) \delta 7.79(\mathrm{~s}, 1 \mathrm{H}), 7.72-7.66(\mathrm{~m}, 2 \mathrm{H}), 7.55(\mathrm{dd}, J=7.8,7.8 \mathrm{~Hz}, 1 \mathrm{H}), 7.39(\mathrm{~d}, J=8.2 \mathrm{~Hz}$, 2H), $7.27(\mathrm{~d}, J=8.2 \mathrm{~Hz}, 2 \mathrm{H}), 2.50(\mathrm{~s}, 3 \mathrm{H}) .{ }^{19} \mathbf{F ~ N M R}\left(376 \mathrm{MHz}, \mathrm{CDCl}_{3}\right) \delta-62.7(\mathrm{~s}, 3 \mathrm{~F}),-88.7(\mathrm{~s}, 2 \mathrm{~F})$. ${ }^{13} \mathrm{C}$ NMR $\left(126 \mathrm{MHz}, \mathrm{CDCl}_{3}\right) \delta 141.8(\mathrm{t}, J=2.0 \mathrm{~Hz}), 138.6(\mathrm{t}, J=29.3 \mathrm{~Hz}), 133.1(\mathrm{t}, J=28.3 \mathrm{~Hz})$, $131.0(\mathrm{q}, J=32.7 \mathrm{~Hz}), 129.3(\mathrm{t}, J=5.3 \mathrm{~Hz}), 129.1,126.8-126.7(\mathrm{~m}), 126.1(\mathrm{t}, J=5.6 \mathrm{~Hz}), 125.8$, $123.7(\mathrm{q}, J=273.1 \mathrm{~Hz}), 122.8-122.6(\mathrm{~m}), 119.9(\mathrm{t}, J=242.4 \mathrm{~Hz}), 15.1$. IR (thin film) $v_{\max } 2925$, $1602,1496 \mathrm{~cm}^{-1}$. MS (EI) (\%) m/z $318\left(\mathrm{M}^{+}, 100\right), 271,195,173$. HRMS (EI) calculated for $\mathrm{C}_{15} \mathrm{H}_{11} \mathrm{~F}_{5} \mathrm{~S}$ : 318.0502; Found: $318.0495\left(\mathrm{M}^{+}\right)$.

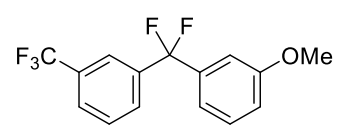

1-(Difluoro(3-(trifluoromethyl)phenyl)methyl)-3-methoxybenzene (3i). The product ( $57 \%$ yield determined by ${ }^{19} \mathrm{~F}$ NMR; $41.8 \mathrm{mg}, 46 \%$ yield) was purified with reverse-phase flash column chromatography $\left(\mathrm{CH}_{3} \mathrm{CN}: \mathrm{H}_{2} \mathrm{O}=6: 4\right)$ as a pale yellow oil. ${ }^{\mathbf{1}} \mathbf{H}$ NMR $\left(400 \mathrm{MHz}, \mathrm{CDCl}_{3}\right) \delta 7.80(\mathrm{~s}, 1 \mathrm{H}), 7.73-7.65(\mathrm{~m}, 2 \mathrm{H}), 7.55(\mathrm{dd}, J=7.8,7.8 \mathrm{~Hz}, 1 \mathrm{H}), 7.35(\mathrm{dd}, J=$ 8.1, 8.1 Hz, 1H), $7.09-7.02(\mathrm{~m}, 2 \mathrm{H}), 7.02-6.95(\mathrm{~m}, 1 \mathrm{H}), 3.83(\mathrm{~s}, 3 \mathrm{H}) .{ }^{19} \mathbf{F}$ NMR $\left(376 \mathrm{MHz}, \mathrm{CDCl}_{3}\right)$ $\delta-62.8(\mathrm{~s}, 3 \mathrm{~F}),-89.3(\mathrm{~s}, 2 \mathrm{~F}) .{ }^{13} \mathbf{C}$ NMR $\left(126 \mathrm{MHz}, \mathrm{CDCl}_{3}\right) \delta 159.7,138.6$ (t, $\left.J=29.2 \mathrm{~Hz}\right), 138.2(\mathrm{t}, J$ $=28.0 \mathrm{~Hz}), 131.0(\mathrm{q}, J=32.9 \mathrm{~Hz}), 129.8,129.3(\mathrm{t}, J=5.5 \mathrm{~Hz}), 129.1,126.8-126.7(\mathrm{~m}), 123.7(\mathrm{q}, J$ $=273.1 \mathrm{~Hz}), 122.8-122.6(\mathrm{~m}), 119.8(\mathrm{t}, J=243.0 \mathrm{~Hz}), 118.0(\mathrm{t}, J=5.6 \mathrm{~Hz}), 115.7,111.4(\mathrm{t}, J=5.9$ Hz), 55.4. IR (thin film) $v_{\max }$ 3009, 2945, 1606, 1491, $1170 \mathrm{~cm}^{-1} . \mathbf{M S}(\mathrm{EI}) \mathrm{m} / \mathrm{z}(\%) 302\left(\mathrm{M}^{+}, 100\right)$, 195, 157, 127, 77. HRMS (EI) calculated for $\mathrm{C}_{15} \mathrm{H}_{11} \mathrm{~F}_{5} \mathrm{O}: 302.0730$; Found: $302.0714\left(\mathrm{M}^{+}\right)$.

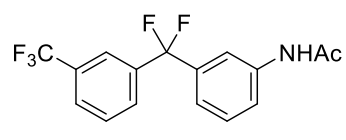

$N$-(3-(Difluoro(3-(trifluoromethyl)phenyl)methyl)phenyl)acetamide $\quad(3 \mathbf{j})$.

The product (40.6 mg, $41 \%$ yield) was purified with reverse-phase preparative HPLC $\left(\mathrm{CH}_{3} \mathrm{CN}: \mathrm{H}_{2} \mathrm{O}=7: 3\right)$ as a pale yellow oil. ${ }^{1} \mathbf{H} \mathbf{N M R}\left(400 \mathrm{MHz}, \mathrm{CDCl}_{3}\right) \delta 7.77(\mathrm{~s}, 1 \mathrm{H}), 7.71-$ $7.63(\mathrm{~m}, 4 \mathrm{H}), 7.61(\mathrm{~s}, 1 \mathrm{H}), 7.52(\mathrm{dd}, J=7.8,7.8 \mathrm{~Hz}, 1 \mathrm{H}), 7.37(\mathrm{dd}, J=8.0,8.0 \mathrm{~Hz}, 1 \mathrm{H}), 7.21(\mathrm{~d}, J=$ 
$7.8 \mathrm{~Hz}, 1 \mathrm{H}), 2.15(\mathrm{~s}, 3 \mathrm{H}) .{ }^{19} \mathbf{F}$ NMR $\left(376 \mathrm{MHz}, \mathrm{CDCl}_{3}\right) \delta-67.5(\mathrm{~s}, 3 \mathrm{~F}),-94.3(\mathrm{~s}, 2 \mathrm{~F}) .{ }^{13} \mathbf{C}$ NMR $(126$ $\left.\mathrm{MHz}, \mathrm{CDCl}_{3}\right) \delta 168.7,138.4(\mathrm{t}, J=28.9 \mathrm{~Hz}), 138.3,137.6(\mathrm{t}, J=28.2 \mathrm{~Hz}), 131.0(\mathrm{q}, J=32.7 \mathrm{~Hz})$, 129.4, $129.2(\mathrm{t}, J=5.4 \mathrm{~Hz}), 129.1,126.9-126.7(\mathrm{~m}), 123.6(\mathrm{q}, J=272.8 \mathrm{~Hz}), 122.7-122.5(\mathrm{~m})$, 121.6, $121.4(\mathrm{t}, J=5.6 \mathrm{~Hz}), 119.6(\mathrm{t}, J=243.3 \mathrm{~Hz}), 116.9(\mathrm{t}, J=6.0 \mathrm{~Hz}), 24.5$. IR (thin film) $v_{\max }$ 3303, 3092, 2926, 1670, 1614, $1131 \mathrm{~cm}^{-1}$. MS (ESI) $(\%) 330(\mathrm{M}+\mathrm{H})^{+}$. HRMS (ESI) calculated for $\mathrm{C}_{16} \mathrm{H}_{12} \mathrm{~F}_{5} \mathrm{NO}$ : 329.0839; Found: $330.0913(\mathrm{M}+\mathrm{H})^{+}$.

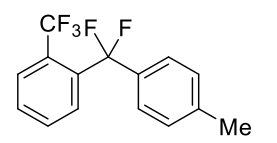

1-(Difluoro(p-tolyl)methyl)-2-(trifluoromethyl)benzene (3k). The product (58\% yield determined by ${ }^{19} \mathrm{~F}$ NMR; $40.8 \mathrm{mg}$, $48 \%$ yield) was purified with reverse-phase preparative HPLC $\left(\mathrm{CH}_{3} \mathrm{CN}: \mathrm{H}_{2} \mathrm{O}=9: 1\right)$ as a pale yellow oil. ${ }^{1} \mathbf{H} \mathbf{N M R}\left(400 \mathrm{MHz}, \mathrm{CDCl}_{3}\right) \delta 7.83(\mathrm{~d}, J$ $=7.7 \mathrm{~Hz}, 1 \mathrm{H}), 7.77(\mathrm{~d}, J=7.7 \mathrm{~Hz}, 1 \mathrm{H}), 7.66(\mathrm{dd}, J=7.7,7.7 \mathrm{~Hz}, 1 \mathrm{H}), 7.60(\mathrm{dd}, J=7.7,7.7 \mathrm{~Hz}, 1 \mathrm{H})$, $7.29(\mathrm{~d}, J=8.0 \mathrm{~Hz}, 2 \mathrm{H}), 7.19(\mathrm{~d}, J=8.0 \mathrm{~Hz}, 2 \mathrm{H}), 2.37(\mathrm{~s}, 3 \mathrm{H}) .{ }^{19}$ F NMR $\left(376 \mathrm{MHz}, \mathrm{CDCl}_{3}\right) \delta-57.8$ (s, 3F), -82.6 (s, 2F). ${ }^{13} \mathrm{C}$ NMR $\left(101 \mathrm{MHz}, \mathrm{CDCl}_{3}\right) \delta 140.2,135.1(\mathrm{t}, J=21.0 \mathrm{~Hz}), 134.6(\mathrm{t}, J=28.1$ Hz), 131.6, 130.2, $129.0(\mathrm{t}, J=8.3 \mathrm{~Hz}), 128.9,128.2(\mathrm{q}, J=32.7 \mathrm{~Hz}), 127.7(\mathrm{q}, J=6.3 \mathrm{~Hz}), 125.8(\mathrm{t}$, $J=5.2 \mathrm{~Hz}), 123.4(\mathrm{q}, J=275.6 \mathrm{~Hz}), 120.3(\mathrm{t}, J=243.4 \mathrm{~Hz}), 21.24$. IR (thin film) $v_{\max } 3042,2927$, 1609, $1516 \mathrm{~cm}^{-1}$. MS (EI) m/z (\%) $286\left(\mathrm{M}^{+}\right), 195,141$ (100). HRMS (EI) calculated for $\mathrm{C}_{15} \mathrm{H}_{11} \mathrm{~F}_{5}$ : 286.0781; Found: $286.0769\left(\mathrm{M}^{+}\right)$.

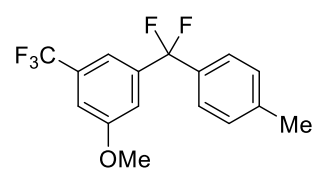

1-(Difluoro(p-tolyl)methyl)-3-methoxy-5-(trifluoromethyl)benzene (31). The product (49\% yield determined by ${ }^{19} \mathrm{~F}$ NMR; $36.3 \mathrm{mg}$, 38\% yield) was purified with reverse-phase preparative HPLC $\left(\mathrm{CH}_{3} \mathrm{CN}: \mathrm{H}_{2} \mathrm{O}=8: 2\right)$ as a pale yellow oil. ${ }^{1} \mathbf{H}$ NMR $\left(400 \mathrm{MHz}, \mathrm{CDCl}_{3}\right) \delta 7.38(\mathrm{~d}, J=8.0 \mathrm{~Hz}, 2 \mathrm{H}), 7.35(\mathrm{~s}, 1 \mathrm{H}), 7.23(\mathrm{~d}, J=8.0 \mathrm{~Hz}, 2 \mathrm{H}), 7.21$ (s, 1H), $7.18(\mathrm{~s}, 1 \mathrm{H}), 3.85(\mathrm{~s}, 3 \mathrm{H}), 2.39(\mathrm{~s}, 3 \mathrm{H}) .{ }^{19} \mathbf{F}$ NMR $\left(376 \mathrm{MHz}, \mathrm{CDCl}_{3}\right) \delta-62.8(\mathrm{~s}, 3 \mathrm{~F}),-88.7$ (s, 2F). ${ }^{13}$ C NMR $\left(126 \mathrm{MHz}, \mathrm{CDCl}_{3}\right) \delta 159.8,140.4(\mathrm{t}, J=1.6 \mathrm{~Hz}), 140.3(\mathrm{t}, J=29.4 \mathrm{~Hz}), 133.9(\mathrm{t}, J=28.1$ $\mathrm{Hz}), 132.2(\mathrm{q}, J=32.9 \mathrm{~Hz}), 129.2,125.6(\mathrm{t}, J=5.5 \mathrm{~Hz}), 123.5(\mathrm{q}, J=272.8 \mathrm{~Hz}), 120.0(\mathrm{t}, J=242.4$ $\mathrm{Hz}), 115.1(\mathrm{t}, J=5.4 \mathrm{~Hz}), 115.0-114.7(\mathrm{~m}), 112.20-112.0(\mathrm{~m}), 55.7,21.3$. IR (thin film) $v_{\max } 3012$, 2928, 1610, 1516, $1131 \mathrm{~cm}^{-1}$. MS (EI) m/z (\%) $316\left(\mathrm{M}^{+}, 100\right), 225,183,141$. HRMS (EI) calculated for $\mathrm{C}_{16} \mathrm{H}_{13} \mathrm{~F}_{5} \mathrm{O}$ : 316.0887 ; Found: $316.0881\left(\mathrm{M}^{+}\right)$. 


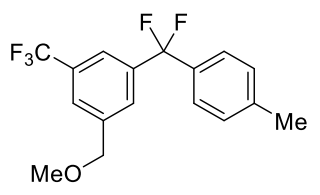

1-(Difluoro(p-tolyl)methyl)-3-(methoxymethyl)-5-(trifluoromethyl)benzene

(3m). 3.0 equiv of trifluoromethylarene was used. The product $(64 \%$ yield determined by ${ }^{19} \mathrm{~F}$ NMR; $45.0 \mathrm{mg}, 45 \%$ yield) was purified with reverse-phase preparative HPLC $\left(\mathrm{CH}_{3} \mathrm{CN}: \mathrm{H}_{2} \mathrm{O}=8: 2\right)$ as a pale yellow oil. ${ }^{1} \mathbf{H}$ NMR $\left(400 \mathrm{MHz}, \mathrm{CDCl}_{3}\right) \delta 7.70(\mathrm{~s}$, 1H), $7.69(\mathrm{~s}, 1 \mathrm{H}), 7.66(\mathrm{~s}, 1 \mathrm{H}), 7.38(\mathrm{~d}, J=8.0 \mathrm{~Hz}, 2 \mathrm{H}), 7.24(\mathrm{~d}, J=8.0 \mathrm{~Hz}, 2 \mathrm{H}), 4.52(\mathrm{~s}, 2 \mathrm{H}), 3.43(\mathrm{~s}$, 3H), 2.39 (s, 3H). ${ }^{19}$ F NMR $\left(376 \mathrm{MHz}, \mathrm{CDCl}_{3}\right) \delta-62.7$ (s, 3F), -88.5 (s, 2F). ${ }^{13} \mathrm{C}$ NMR $(101 \mathrm{MHz}$, $\left.\mathrm{CDCl}_{3}\right) \delta 140.4,140.2,139.1(\mathrm{t}, J=29.3 \mathrm{~Hz}), 134.0(\mathrm{t}, J=28.0 \mathrm{~Hz}), 131.2(\mathrm{q}, J=32.8 \mathrm{~Hz}), 129.2$, 127.9, $125.6(\mathrm{t}, J=5.6 \mathrm{~Hz}), 125.6-125.3(\mathrm{~m}), 123.7(\mathrm{q}, J=273.9 \mathrm{~Hz}), 122.1-121.7(\mathrm{~m}), 120.1(\mathrm{t}, J$ $=243.5 \mathrm{~Hz}), 73.5,58.5,21.2 . \mathbf{I R}\left(\right.$ thin film) $v_{\max } 2995,2929,1616,1516,1131 \mathrm{~cm}^{-1} . \mathbf{M S}(\mathrm{EI}) \mathrm{m} / \mathrm{z}(\%)$ $330\left(\mathrm{M}^{+}\right), 299,267,223,189$ (100), 141. HRMS (EI) calculated for $\mathrm{C}_{8} \mathrm{H}_{15} \mathrm{~F}_{5} \mathrm{O}$ : 330.1043; Found: $330.1039\left(\mathrm{M}^{+}\right)$.

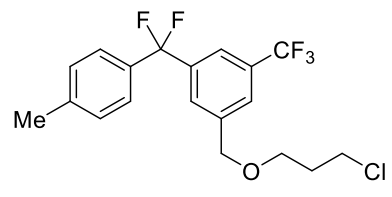

\section{1-((3-Chloropropoxy)methyl)-3-(difluoro(p-tolyl)methyl)-5-}

(trifluoromethyl)benzene (3n). The product $\left(50 \%\right.$ yield determined by ${ }^{19} \mathrm{~F}$

NMR; $52.2 \mathrm{mg}, 44 \%$ yield) was purified with reverse-phase flash column chromatography $\left(\mathrm{CH}_{3} \mathrm{CN}: \mathrm{H}_{2} \mathrm{O}=6: 4\right)$ as a pale yellow oil. ${ }^{\mathbf{1}} \mathbf{H} \mathbf{~ N M R}\left(400 \mathrm{MHz}, \mathrm{CDCl}_{3}\right){ }^{1} \mathrm{H}$ NMR (400 MHz, Chloroform- $d$ ) $\delta 7.69(\mathrm{~s}, 1 \mathrm{H}), 7.66(\mathrm{~s}, 1 \mathrm{H}), 7.63(\mathrm{~s}, 1 \mathrm{H}), 7.37(\mathrm{~d}, J=7.8 \mathrm{~Hz}, 2 \mathrm{H}), 7.23$ $(\mathrm{d}, J=7.7 \mathrm{~Hz}, 2 \mathrm{H}), 4.57(\mathrm{~s}, 2 \mathrm{H}), 3.71-3.60(\mathrm{~m}, 4 \mathrm{H}), 2.38(\mathrm{~s}, 3 \mathrm{H}), 2.06(\mathrm{tt}, J=6.1,6.1 \mathrm{~Hz}, 2 \mathrm{H})$. ${ }^{19} \mathbf{F}$ NMR $\left(376 \mathrm{MHz}, \mathrm{CDCl}_{3}\right) \delta-62.6(\mathrm{~s}, 3 \mathrm{~F}),-88.5(\mathrm{~s}, 2 \mathrm{~F}) .{ }^{13} \mathrm{C} \mathrm{NMR}\left(126 \mathrm{MHz}, \mathrm{CDCl}_{3}\right) \delta 140.4$, 140.2, $139.1(\mathrm{t}, J=29.4 \mathrm{~Hz}), 133.9(\mathrm{t}, J=28.0 \mathrm{~Hz}), 131.2(\mathrm{q}, J=32.8 \mathrm{~Hz}), 129.2,127.9(\mathrm{t}, J=4.8$ $\mathrm{Hz}), 125.6(\mathrm{t}, J=5.6 \mathrm{~Hz}), 125.4-125.3(\mathrm{~m}), 123.6(\mathrm{q}, J=272.6 \mathrm{~Hz}), 122.0-121.8(\mathrm{~m}), 120.1(\mathrm{t}$, $J=242.2 \mathrm{~Hz}), 71.9,67.2,41.7,32.5,21.2 . \mathbf{I R}\left(\right.$ thin film) $v_{\max } 2925,1618,1459 \mathrm{~cm}^{-1} . \mathbf{M S}(\mathrm{EI}) \mathrm{m} / \mathrm{z}$

(\%) $392\left(\mathrm{M}^{+}\right), 299$ (100), 267, 208. HRMS (EI) calculated for $\mathrm{C}_{19} \mathrm{H}_{18} \mathrm{ClF}_{5} \mathrm{O}$ : 392.0966; Found: $392.0964\left(\mathrm{M}^{+}\right)$.

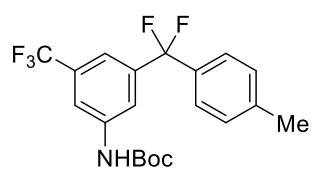

tert-Butyl (3-(difluoro(p-tolyl)methyl)-5-(trifluoromethyl)phenyl)carbamate (3o). 3.0 equiv of trifluoromethylarene was used. The product $(56.2 \mathrm{mg}, 47 \%$ yield) was purified by flash column chromatography on silica gel (1-1.5\% EA/PE) as a colorless oil. ${ }^{1} \mathbf{H}$ NMR $\left(400 \mathrm{MHz}, \mathrm{CDCl}_{3}\right) \delta 7.89(\mathrm{~s}, 1 \mathrm{H}), 7.56(\mathrm{~s}, 1 \mathrm{H}), 7.43(\mathrm{~s}, 1 \mathrm{H}), 7.37(\mathrm{~d}, J=$ 
$7.8 \mathrm{~Hz}, 2 \mathrm{H}), 7.22(\mathrm{~d}, J=7.8 \mathrm{~Hz}, 2 \mathrm{H}), 6.80(\mathrm{~s}, 1 \mathrm{H}), 2.38(\mathrm{~s}, 3 \mathrm{H}), 1.52(\mathrm{~s}, 9 \mathrm{H}) .{ }^{19} \mathbf{F}$ NMR $(376 \mathrm{MHz}$, $\left.\mathrm{CDCl}_{3}\right) \delta-62.9(\mathrm{~s}, 3 \mathrm{~F}),-88.9(\mathrm{~s}, 2 \mathrm{~F}) .{ }^{13} \mathrm{C} \mathrm{NMR}\left(126 \mathrm{MHz}, \mathrm{CDCl}_{3}\right) \delta 152.3,140.4(\mathrm{t}, J=1.4 \mathrm{~Hz}), 139.7$ $(\mathrm{t}, J=29.5 \mathrm{~Hz}), 139.4,133.9(\mathrm{t}, J=28.0 \mathrm{~Hz}), 131.8(\mathrm{q}, J=32.8 \mathrm{~Hz}), 129.2,125.6(\mathrm{t}, J=5.5 \mathrm{~Hz}), 123.5$ $(\mathrm{q}, J=272.7 \mathrm{~Hz}), 119.9(\mathrm{t}, J=242.6 \mathrm{~Hz}), 118.8-118.5(\mathrm{~m}), 116.9-116.6(\mathrm{~m}), 116.4-116.2(\mathrm{~m})$, 81.5, 28.2, 21.2. IR (thin film) $v_{\max } 327,2982,1705,1618,1541,1133 \mathrm{~cm}^{-1}$. MS (ESI) (\%) 324 (MBoc $+\mathrm{Na})^{+}$. HRMS (ESI) calculated for $\mathrm{C}_{20} \mathrm{H}_{20} \mathrm{~F}_{5} \mathrm{NO}_{2}$ : 401.1414; Found: $424.1309(\mathrm{M}+\mathrm{Na})^{+}$.

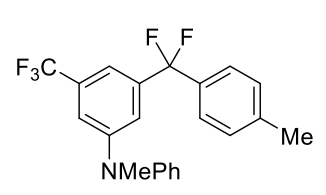

\section{3-(Difluoro(p-tolyl)methyl)- $N$-methyl- $N$-phenyl-5-(trifluoromethyl)aniline}

(3p). 3.0 equiv of trifluoromethylarene was used. The product $(60.1 \mathrm{mg}, 51 \%$ yield) was purified with reverse-phase preparative $\mathrm{HPLC}\left(\mathrm{CH}_{3} \mathrm{CN}: \mathrm{H}_{2} \mathrm{O}=9: 1\right)$ as a yellow oil. ${ }^{1} \mathbf{H}$ NMR (400 MHz, $\left.\mathrm{CDCl}_{3}\right) \delta 7.42-7.34(\mathrm{~m}, 4 \mathrm{H}), 7.25-7.12(\mathrm{~m}, 7 \mathrm{H}), 7.09$ (s, 1H), $3.35(\mathrm{~s}, 3 \mathrm{H}), 2.39$ (s, 3H). ${ }^{19} \mathbf{F}$ NMR $\left(376 \mathrm{MHz}, \mathrm{CDCl}_{3}\right) \delta-62.8(\mathrm{~s}, 3 \mathrm{~F}),-88.9(\mathrm{~s}, 2 \mathrm{~F}) .{ }^{\mathbf{1 3}} \mathbf{C}$ NMR $(126$ $\left.\mathrm{MHz} \mathrm{CDCl}_{3}\right) \delta 149.4,147.4,140.2,139.6(\mathrm{t}, J=28.9 \mathrm{~Hz}), 134.2(\mathrm{t}, J=28.4 \mathrm{~Hz}), 131.6(\mathrm{q}, J=32.2$ Hz), 129.9, 129.1, 125.6 (t, $J=5.5 \mathrm{~Hz}), 124.9,124.6,123.8$ (q, $J=273.1 \mathrm{~Hz}), 120.3$ (t, $J=242.3 \mathrm{~Hz})$, $115.9(\mathrm{t}, J=5.7 \mathrm{~Hz}), 113.5-113.4(\mathrm{~m}), 112.5-112.3(\mathrm{~m}), 40.3,21.2 . \mathbf{I R}\left(\right.$ thin film) $v_{\max } 3037,2925$, 1617, 1593, 1497, $1128 \mathrm{~cm}^{-1}$. MS (ESI) (\%) $392(\mathrm{M}+\mathrm{H})^{+}$. HRMS (ESI) calculated for $\mathrm{C}_{22} \mathrm{H}_{18} \mathrm{~F}_{5} \mathrm{~N}$ : 391.1359; Found: $392.1433(\mathrm{M}+\mathrm{H})^{+}$.

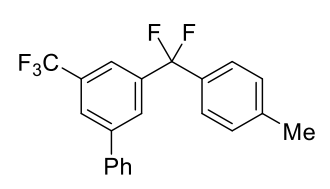

3-(Difluoro(p-tolyl)methyl)-5-(trifluoromethyl)-1,1'-biphenyl (3q). 3.0 equiv of trifluoromethylarene was used. The product $(59.9 \mathrm{mg}$, 55\% yield) was purified with reverse-phase preparative HPLC $\left(\mathrm{CH}_{3} \mathrm{CN}: \mathrm{H}_{2} \mathrm{O}=8: 2\right)$ as a pale yellow oil. ${ }^{1} \mathbf{H}$ NMR $\left(400 \mathrm{MHz}, \mathrm{CDCl}_{3}\right) \delta 7.92(\mathrm{~s}, 2 \mathrm{H}), 7.78(\mathrm{~s}, 1 \mathrm{H}), 7.60$ (d, $\left.J=7.7 \mathrm{~Hz}, 2 \mathrm{H}\right), 7.50$ (dd, $J=7.7$, $7.7 \mathrm{~Hz}, 2 \mathrm{H}), 7.46-7.40(\mathrm{~m}, 3 \mathrm{H}), 7.26(\mathrm{~d}, J=7.7 \mathrm{~Hz}, 2 \mathrm{H}), 2.40(\mathrm{~s}, 3 \mathrm{H}) .{ }^{19} \mathbf{F} \mathbf{N M R}\left(376 \mathrm{MHz}, \mathrm{CDCl}_{3}\right)$ $\delta-62.6(\mathrm{~s}, 3 \mathrm{~F}),-88.5(\mathrm{~s}, 2 \mathrm{~F}) .{ }^{13} \mathrm{C}$ NMR $\left(126 \mathrm{MHz}, \mathrm{CDCl}_{3}\right) \delta 142.6,140.4,139.5(\mathrm{t}, J=29.3 \mathrm{~Hz}), 138.9$, $133.9(\mathrm{t}, J=28.1 \mathrm{~Hz}), 131.5(\mathrm{q}, J=32.6 \mathrm{~Hz}), 129.3,129.1,128.5,127.9(\mathrm{t}, J=5.3 \mathrm{~Hz}), 127.2,125.7$ $(\mathrm{t}, J=5.5 \mathrm{~Hz}), 125.4-125.3(\mathrm{~m}), 123.7(\mathrm{q}, J=272.6 \mathrm{~Hz}), 121.5-121.3(\mathrm{~m}), 120.2(\mathrm{t}, J=242.9 \mathrm{~Hz})$, 21.3. IR (thin film) $v_{\max } 3038,2925,1615,1516 \mathrm{~cm}^{-1}$. MS (EI) m/z (\%) $362\left(\mathrm{M}^{+}, 100\right), 271,201,141$. HRMS (EI) calculated for $\mathrm{C}_{21} \mathrm{H}_{15} \mathrm{~F}_{5}$ : 362.1094; Found: $362.1080\left(\mathrm{M}^{+}\right)$. 


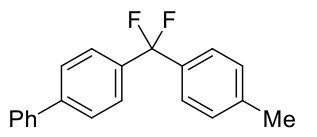

trifluoromethylarene was used. The product ( $36.2 \mathrm{mg}, 41 \%$ yield) was purified with reverse-phase preparative $\mathrm{HPLC}\left(\mathrm{CH}_{3} \mathrm{CN}: \mathrm{H}_{2} \mathrm{O}=7: 3\right)$ as a white solid (m.p. 123-126 $\left.{ }^{\circ} \mathrm{C}\right) .{ }^{1} \mathbf{H}$ NMR $\left(400 \mathrm{MHz}, \mathrm{CDCl}_{3}\right) \delta 7.64(\mathrm{~d}, J=8.2 \mathrm{~Hz}, 2 \mathrm{H}), 7.62-7.56(\mathrm{~m}, 4 \mathrm{H}), 7.50-7.42(\mathrm{~m}, 4 \mathrm{H}), 7.39$ $(\mathrm{dd}, J=7.3,7.3 \mathrm{~Hz}, 1 \mathrm{H}), 7.25(\mathrm{~d}, J=8.2 \mathrm{~Hz}, 2 \mathrm{H}), 2.40(\mathrm{~s}, 3 \mathrm{H}),{ }^{19} \mathbf{F} \mathbf{N M R}\left(376 \mathrm{MHz}, \mathrm{CDCl}_{3}\right) \delta-62.4$ ${ }^{13} \mathrm{C}$ NMR $\left(126 \mathrm{MHz}, \mathrm{CDCl}_{3}\right) \delta 142.7,140.2,139.9,136.7(\mathrm{t}, J=28.8 \mathrm{~Hz}), 134.8(\mathrm{t}, J=28.4 \mathrm{~Hz})$, 129.0, 128.8, 127.7, 127.2, 127.1, $126.3(\mathrm{t}, J=5.4 \mathrm{~Hz}), 125.8(\mathrm{t}, J=5.4 \mathrm{~Hz}), 120.9(\mathrm{t}, J=241.2 \mathrm{~Hz})$, 21.3. IR (thin film) $v_{\max } 3077,2923,1612,1515 \mathrm{~cm}^{-1} . \mathbf{M S}(\mathrm{EI}) \mathrm{m} / \mathrm{z}(\%) 294\left(\mathrm{M}^{+}, 100\right), 203,183,141$. HRMS (EI) calculated for $\mathrm{C}_{20} \mathrm{H}_{16} \mathrm{~F}_{2}$ : 294.1220; Found: $294.1208\left(\mathrm{M}^{+}\right)$.

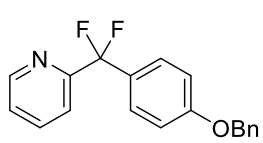

2-((4-(Benzyloxy)phenyl)difluoromethyl)pyridine $\quad$ (3s). $3.0 \quad$ equiv of trifluoromethylarene was used. The product (56\% yield determined by ${ }^{19} \mathrm{~F}$ NMR; $46.5 \mathrm{mg}, 50 \%$ yield) was purified by flash column chromatography on silica gel (5-10\% EA/PE) as a white solid (m.p. $\left.84-87^{\circ} \mathrm{C}\right) .{ }^{1} \mathbf{H}$ NMR $\left(400 \mathrm{MHz}, \mathrm{CDCl}_{3}\right) \delta 8.67(\mathrm{~d}, J=4.8 \mathrm{~Hz}, 1 \mathrm{H}), 7.80(\mathrm{dd}, J=7.7$, $7.7 \mathrm{~Hz}, 1 \mathrm{H}), 7.72(\mathrm{~d}, J=7.9 \mathrm{~Hz}, 1 \mathrm{H}), 7.53(\mathrm{~d}, J=8.4 \mathrm{~Hz}, 2 \mathrm{H}), 7.46-7.30(\mathrm{~m}, 6 \mathrm{H}), 7.01(\mathrm{~d}, J=8.4$ $\mathrm{Hz}, 2 \mathrm{H}), 5.08(\mathrm{~s}, 2 \mathrm{H}) .{ }^{19} \mathbf{F}$ NMR $\left(376 \mathrm{MHz}, \mathrm{CDCl}_{3}\right) \delta-92.3 .{ }^{13} \mathbf{C ~ N M R}\left(126 \mathrm{MHz}, \mathrm{CDCl}_{3}\right) \delta 159.9$, $155.6(\mathrm{t}, J=31.1 \mathrm{~Hz}), 149.6,137.0,136.5,129.0(\mathrm{t}, J=27.9 \mathrm{~Hz}), 128.6,128.0,127.4,127.4(\mathrm{t}, J=5.7$ Hz), 124.4, $120.1(\mathrm{t}, J=4.3 \mathrm{~Hz}), 118.8(\mathrm{t}, J=242.7 \mathrm{~Hz}), 114.5,70.0$. IR (thin film) $v_{\max } 3033,1611$, 1590, $1514 \mathrm{~cm}^{-1}$. MS (ESI) (\%) $312(\mathrm{M}+\mathrm{H})^{+}$. HRMS (ESI) calculated for $\mathrm{C}_{19} \mathrm{H}_{15} \mathrm{~F}_{2} \mathrm{NO}$ : 311.1122 Found: $312.1192(\mathrm{M}+\mathrm{H})^{+}$.

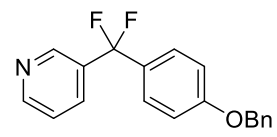

3-((4-(Benzyloxy)phenyl)difluoromethyl)pyridine $\quad(3 t) . \quad 3.0 \quad$ equiv of trifluoromethylarene was used. The product ( $41 \%$ yield determined by ${ }^{19} \mathrm{~F}$ NMR; $32.9 \mathrm{mg}, 35 \%$ yield) was purified by flash column chromatography on silica gel (7.5-15\% EA/PE) as a pale yellow solid (m.p. $\left.73-76{ }^{\circ} \mathrm{C}\right) .{ }^{1} \mathbf{H}$ NMR $\left(400 \mathrm{MHz}, \mathrm{CDCl}_{3}\right) 8.80-8.65(\mathrm{~m}, 2 \mathrm{H}), 7.81(\mathrm{~d}, J=8.0$ $\mathrm{Hz}, 1 \mathrm{H}), 7.48-7.31(\mathrm{~m}, 8 \mathrm{H}), 7.01(\mathrm{~d}, J=8.4 \mathrm{~Hz}, 2 \mathrm{H}), 5.09(\mathrm{~s}, 2 \mathrm{H}) .{ }^{19} \mathbf{F} \mathbf{N M R}\left(376 \mathrm{MHz}, \mathrm{CDCl}_{3}\right) \delta-$ 86.6. ${ }^{13} \mathrm{C} \mathrm{NMR}\left(126 \mathrm{MHz}, \mathrm{CDCl}_{3}\right) \delta 160.1,151.0,147.4(\mathrm{t}, J=5.7 \mathrm{~Hz}), 136.3,133.6(\mathrm{t}, J=29.4 \mathrm{~Hz})$, $133.5(\mathrm{t}, J=5.1 \mathrm{~Hz}), 129.0(\mathrm{t}, J=28.2 \mathrm{~Hz}), 128.6,128.1,127.4,127.3(\mathrm{t}, J=5.5 \mathrm{~Hz}), 123.1,120.0(\mathrm{t}$, 
$J=241.5 \mathrm{~Hz}), 114.8,70.1$. IR (thin film) $v_{\max } 3065,1611,1591,1514 \mathrm{~cm}^{-1} . \mathbf{M S}(\mathrm{ESI})(\%) 312(\mathrm{M}+\mathrm{H})^{+}$. HRMS (ESI) calculated for $\mathrm{C}_{19} \mathrm{H}_{15} \mathrm{~F}_{2} \mathrm{NO}$ : 311.1122; Found: $312.1193(\mathrm{M}+\mathrm{H})^{+}$.

\section{$4.31 \mathrm{mmol}-\mathrm{scale}$ synthesis of $3 \mathrm{~d}$}

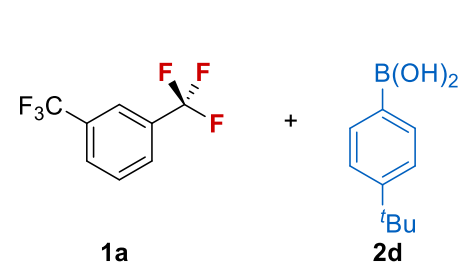

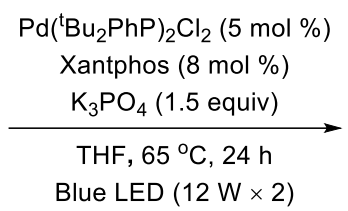

$1 \mathrm{mmol}$ scale

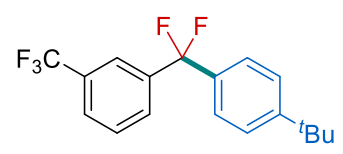

$3 d, 69 \%(52 \%)$

To a $25 \mathrm{~mL}$ of Schlenk tube were added $\mathrm{Pd}\left({ }^{t} \mathrm{Bu}_{2} \mathrm{PhP}\right)_{2} \mathrm{Cl}_{2}(31.1 \mathrm{mg}, 0.05 \mathrm{mmol}, 5 \mathrm{~mol} \%)$, Xantphos (46.3 mg, $0.08 \mathrm{mmol}, 8 \mathrm{~mol} \%)$, and $\mathrm{ArB}(\mathrm{OH})_{2}\left(178 \mathrm{mg}, 1.0 \mathrm{mmol}, 1.0\right.$ equiv) in the air. $\mathrm{K}_{3} \mathrm{PO}_{4}(318.5$ $\mathrm{mg}, 1.5 \mathrm{mmol}, 1.5$ equiv) was added in the glovebox. The reaction mixture was then evacuated and backfilled with $\operatorname{Ar}$ (3 times). $\operatorname{ArCF}_{3}(0.93 \mathrm{~mL}, 6 \mathrm{mmol}, 6$ equiv) and THF (6.7 mL) were added. After pre-stirring at $65^{\circ} \mathrm{C}$ for $20 \mathrm{~min}$, the reaction mixture was irradiated under blue LED strips $(12 \mathrm{~W} \times 2)$ for $24 \mathrm{~h}$ at $65^{\circ} \mathrm{C}$ with stirring. The reaction mixture was then cooled to room temperature and the yield was determined by ${ }^{19} \mathrm{~F}$ NMR using fluorobenzene as an internal standard. The resulting mixture was filtered with a pad of celite. The filtrate was concentrated. The product $\left(69 \%\right.$ yield determined by ${ }^{19} \mathrm{~F}$ NMR; $170 \mathrm{mg}, 52 \%$ yield) was purified with reverse-phase flash column chromatography $\left(\mathrm{CH}_{3} \mathrm{CN}: \mathrm{H}_{2} \mathrm{O}=9: 1\right)$ as a pale yellow oil. 


\subsection{Reaction of 1 equiv of $\mathrm{ArCF}_{3}$ with arylboronic acids}
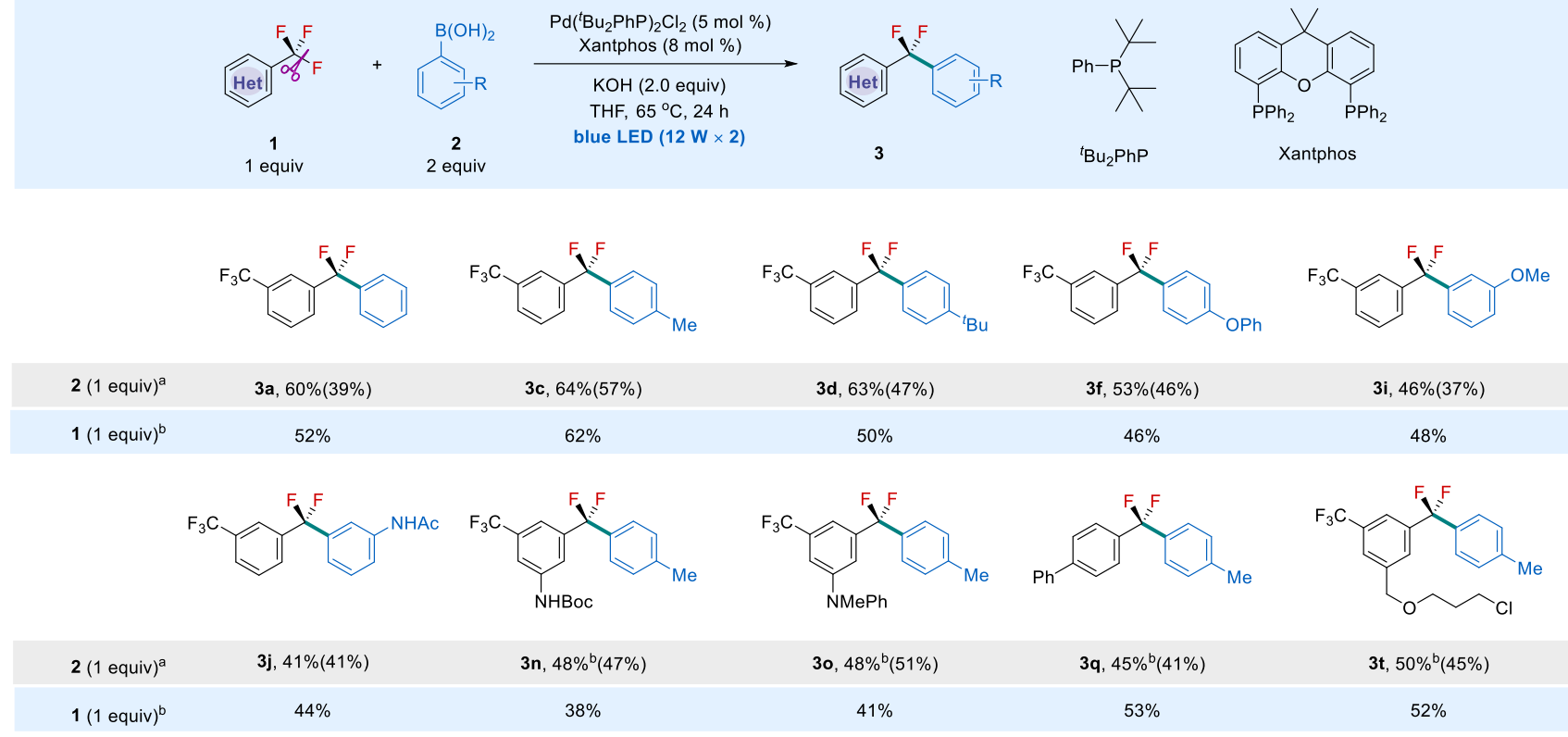

${ }^{a}$ Standard conditions: 1 (3-6 equiv), 2 ( 0.3 mmol, 1.0 equiv), $\mathrm{K}_{3} \mathrm{PO}_{4}$ (1.5 equiv). ${ }^{b}$ Reaction conditions: 1a ( 0.3 mmol, 1.0 equiv), 2a (2.0 equiv), $\mathrm{KOH}$ ( 2 equiv). The yield was determined by ${ }^{19} \mathrm{~F}$ NMR using fluorobenzene as an internal standard; the number given in parentheses is the isolated yield.

Procedure: To a $25 \mathrm{~mL}$ of Schlenk tube were added $\mathrm{Pd}\left({ }^{t} \mathrm{Bu}_{2} \mathrm{PhP}\right)_{2} \mathrm{Cl}_{2}(9.3 \mathrm{mg}, 0.015 \mathrm{mmol}, 5 \mathrm{~mol} \%)$, Xantphos (13.9 mg, $0.024 \mathrm{mmol}, 8 \mathrm{~mol} \%)$, and $\mathrm{ArB}(\mathrm{OH})_{2}(0.6 \mathrm{mmol}, 2.0$ equiv) in the air. $\mathrm{KOH}(33.7$ $\mathrm{mg}, 0.6 \mathrm{mmol}, 2$ equiv) was added in the glovebox. The reaction mixture was then evacuated and backfilled with $\operatorname{Ar}(3$ times $)$. $\operatorname{ArCF}_{3}(0.3 \mathrm{mmol}, 1$ equiv) and THF $(2.0 \mathrm{~mL})$ were added. After prestirring at $65{ }^{\circ} \mathrm{C}$ for $20 \mathrm{~min}$, the reaction mixture was irradiated under blue LED strips $(12 \mathrm{~W} \times 2)$ for $24 \mathrm{~h}$ at $65{ }^{\circ} \mathrm{C}$ with stirring. The reaction mixture was then cooled to room temperature and the yield was determined by ${ }^{19} \mathrm{~F}$ NMR using fluorobenzene as an internal standard. 


\section{Mechanistic Studies}

\subsection{CV measurement of catalyst, trifluoromethylarenes and 3a}

\section{CV measurement of palladium catalyst}

Cyclic voltammograms were recorded with a CHI600D potentiostat at room temperature in degassed THF solution in the glovebox $\left(\left[\mathrm{n}-\mathrm{Bu}_{4} \mathrm{NPF}_{6}\right]=0.1 \mathrm{M},[\mathrm{Pd}]=1.2 \mathrm{mM}\right.$, referenced by $\mathrm{CoCp}_{2} \mathrm{PF}_{6}$ ). A Glass Carbon electrode was used as the working electrode and the auxiliary electrode was a Pt sheet. A silver wire in $0.1 \mathrm{M} \mathrm{AgNO}_{3}$ in $\mathrm{MeCN}$ was used as reference electrode. The scan rate was $100 \mathrm{mV} \mathrm{s}^{-1}$. Potentials vs. $\mathrm{Fc}^{+} / \mathrm{Fc}$ were reported according to $\mathrm{E}_{\mathrm{Fc}+/ \mathrm{Fc}}=\mathrm{E}_{\mathrm{Cc}+/ \mathrm{Cc}}-1.36 \mathrm{~V} .(\mathrm{Cc}=$ cobaltocene).

\section{$\mathrm{FeCp}_{2}+\mathrm{CoCp}_{2} \mathrm{PF}_{6}$}

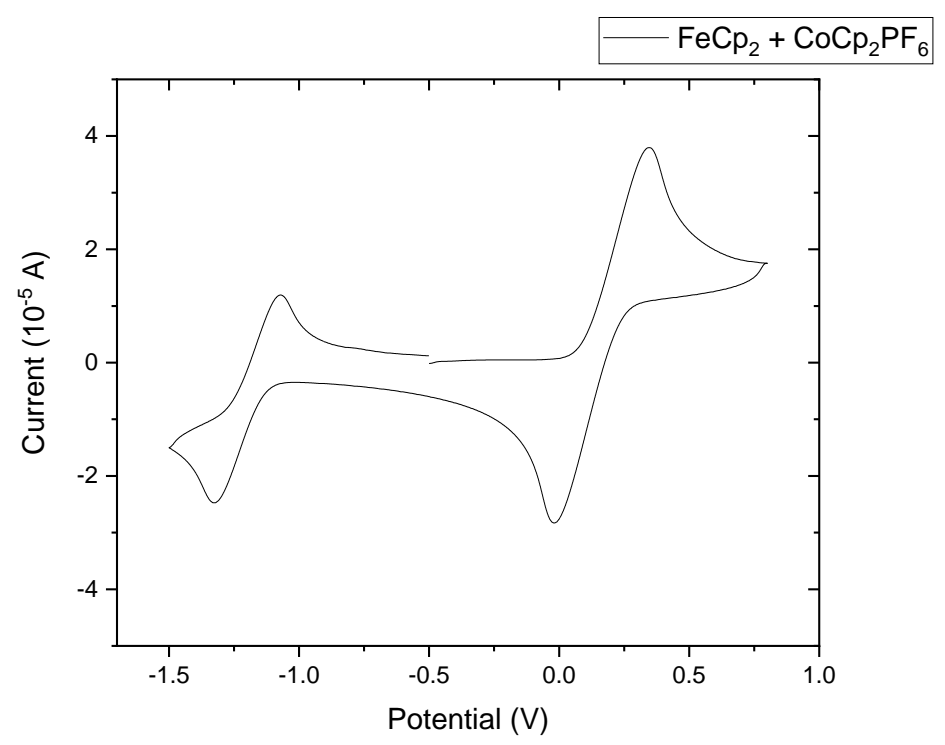

Figure S1. Cyclic Voltammogram of the mixtures of $\mathrm{FeCp}_{2}$ and $\mathrm{CoCp}_{2} \mathrm{PF}_{6}$ in THF $\mathrm{E}_{1 / 2}\left(\mathrm{Cc}^{+} / \mathrm{Cc}\right)=-1.36 \mathrm{~V}$ vs $\mathrm{Fc}^{+} / \mathrm{Fc}$ in $\mathrm{THF}$ 


\section{$\mathbf{P d}\left(\mathbf{P P h}_{3}\right)_{4}$}

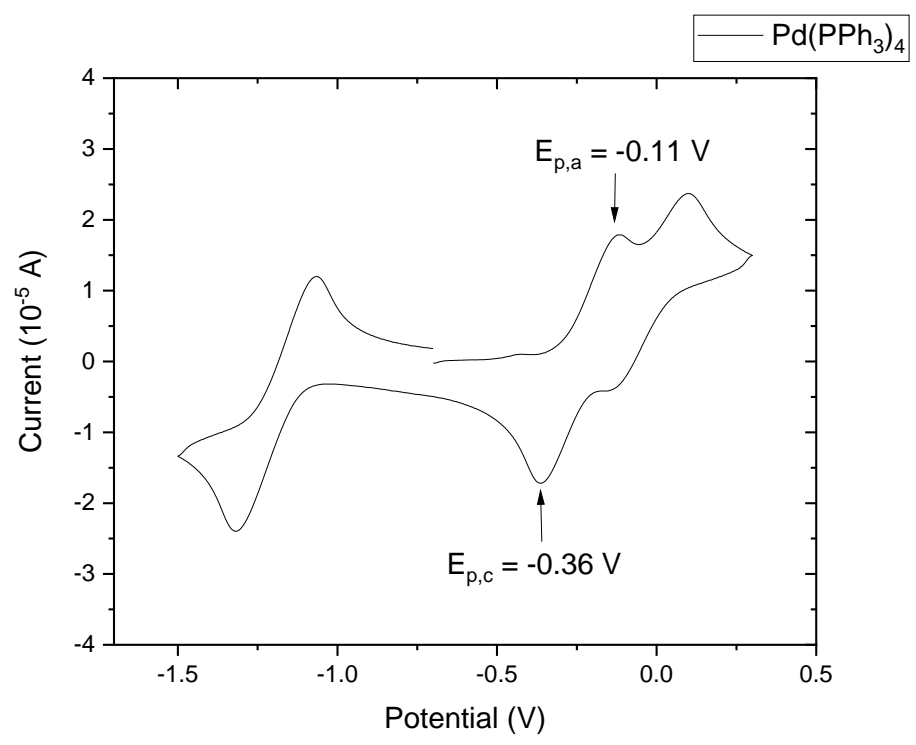

Figure S2. Cyclic Voltammogram of $\mathrm{Pd}\left(\mathrm{PPh}_{3}\right)_{4}$ in $\mathrm{THF}$

$\mathrm{E}_{1 / 2}\left([\mathrm{Pd}]^{\mathrm{I}} /\left[\mathrm{Pd}\left(\mathrm{PPh}_{3}\right)_{4}\right]\right)=-0.41 \mathrm{~V}$ vs $\mathrm{Fc}^{+} / \mathrm{Fc}$ in $\mathrm{THF}$

\section{Pd(PPh3)4 + Xantphos}

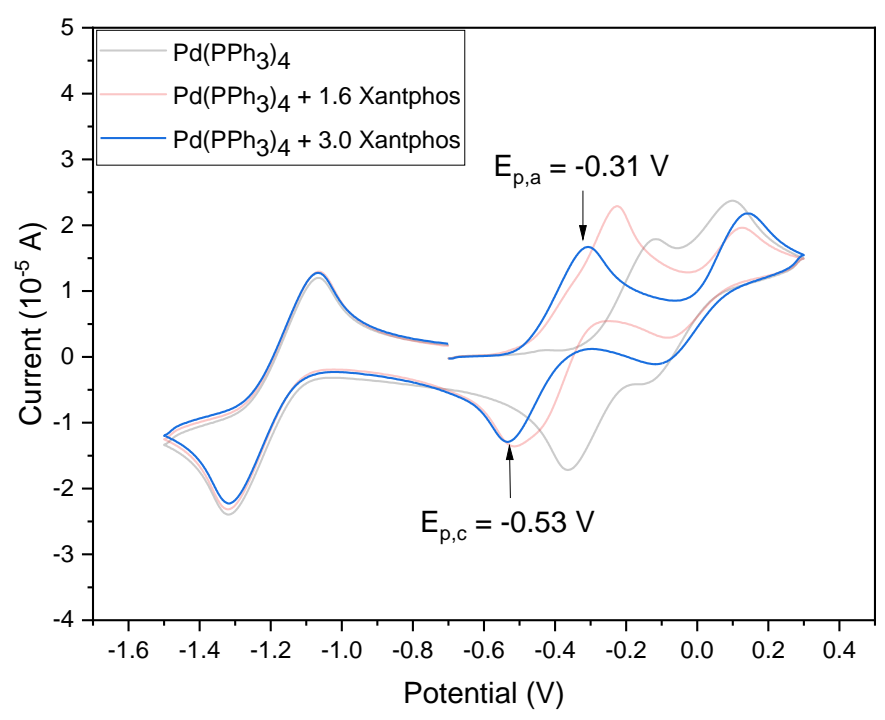

Figure S3. Cyclic Voltammogram of the $\mathrm{Pd}(\text { Xantphos })_{2}$ in THF

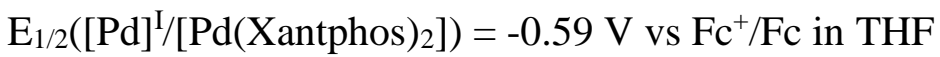




\section{CV measurement of trifluoromethylarenes and 3a}

Cyclic voltammograms were recorded with a CHI760E potentiostat at room temperature in degassed MeCN solution $\left(\left[n-\mathrm{Bu}_{4} \mathrm{NPF}_{6}\right]=0.1 \mathrm{M}\right.$, [substrate $]=5 \mathrm{mM}$, referenced by ferrocene $) . A$ Glass Carbon electrode was used as the working electrode and the auxiliary electrode was a Pt sheet. A silver wire in $0.1 \mathrm{M} \mathrm{AgNO}_{3}$ in $\mathrm{MeCN}$ was used as reference electrode. The scan rate was 100 $\mathrm{mV} \mathrm{s}^{-1}$.

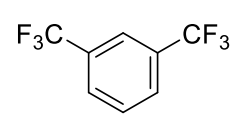

$$
\begin{gathered}
1 a \\
E_{p / 2}=-2.68 \mathrm{~V}
\end{gathered}
$$<smiles>O=C(O)OC(=O)Nc1cc(C(F)(F)F)cc(C(F)(F)F)c1</smiles>

10

$E_{p / 2}=-2.55 \mathrm{~V}$<smiles>FC(F)(F)c1cccnc1</smiles>$$
\begin{gathered}
\mathbf{1 t} \\
E_{p / 2}=-2.66 \mathrm{~V}
\end{gathered}
$$<smiles>FC(F)(F)c1ccccc1</smiles>

$1 \mathbf{y}$

$E_{p / 2}=-3.04 V$<smiles>FC(F)(F)c1ccccc1C(F)(F)F</smiles>

$1 \mathrm{k}$

$$
E_{p / 2}=-2.57 \mathrm{~V}
$$<smiles>CN(c1ccccc1)c1cc(C(F)(F)F)cc(C(F)(F)F)c1</smiles>

$1 \mathrm{p}$

$E_{p / 2}=-2.63 \mathrm{~V}$<smiles>O=C(c1cccc(C(F)(F)F)c1)N1CCOCC1</smiles>

$1 \mathrm{u}$

$E_{p / 2}=-2.60 \mathrm{~V}$<smiles>COc1cccc(C(F)(F)F)c1</smiles>

$\mathbf{z}$ $E_{p / 2}=-3.02 \mathrm{~V}$<smiles>COc1cc(C(F)(F)F)cc(C(F)(F)F)c1</smiles>

11

$E_{p / 2}=-2.64 \mathrm{~V}$<smiles>FC(F)(F)c1cc(-c2ccccc2)cc(C(F)(F)F)c1</smiles>

$1 q$

$E_{p / 2}=-2.53 \mathrm{~V}$<smiles>CCNS(=O)(=O)c1cccc(C(F)(F)F)c1</smiles>

$1 \mathrm{v}$

$E_{p / 2}=-2.39 \mathrm{~V}$<smiles>FC(F)(F)c1cccc(C(F)(F)F)c1</smiles>

$3 a$

$E_{p / 2}=-2.68 \mathrm{~V}$

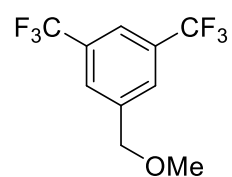

m

$E_{p / 2}=-2.64 \mathrm{~V}$<smiles>FC(F)(F)c1cc(COCCCCl)cc(C(F)(F)F)c1</smiles>

$1 n$

$E_{p / 2}=-2.62 \mathrm{~V}$
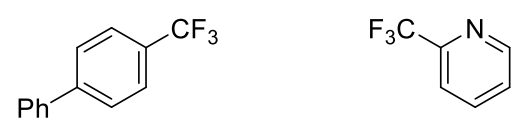

$1 \mathbf{s}$

$E_{p / 2}=-2.63 V$

$E_{p / 2}=-2.67 \mathrm{~V}$<smiles>N#Cc1cccc(C(F)(F)F)c1</smiles><smiles>N#Cc1ccc(C(F)(F)F)cc1</smiles>

$\stackrel{1 w}{1 w} E_{p / 2}=-2.44 \mathrm{~V}$

$E_{p / 2}=-2.27 V$

Half-peak reduction potential of 1 and $3 \mathrm{a} v \mathrm{Fc}^{+} / \mathrm{Fc}$ in $\mathrm{MeCN}$ 

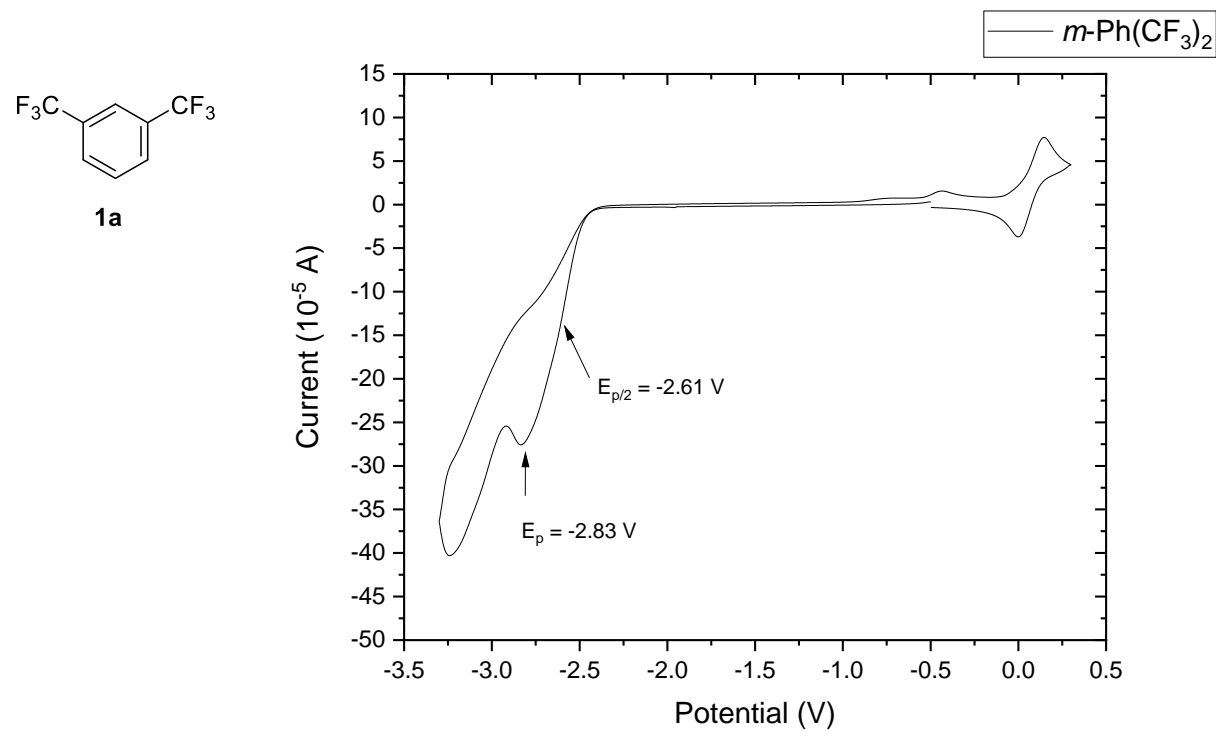

Figure S4. Cyclic Voltammogram of the 1a in $\mathrm{MeCN}$.

$\mathrm{E}_{\mathrm{p} / 2}\left(\mathbf{1 a} / \mathbf{1} \mathbf{a}^{\bullet-}\right)=-2.68 \mathrm{~V}$ vs Fc$+\mathrm{Fc}$ in $\mathrm{MeCN}$.
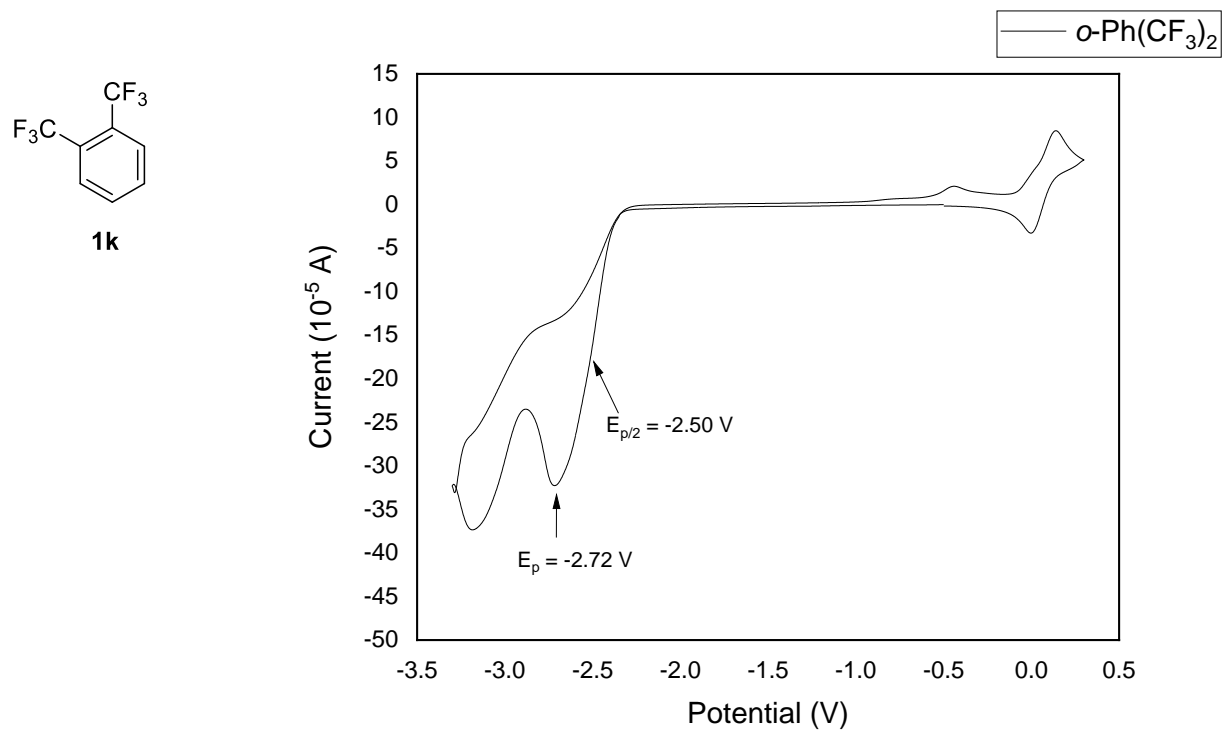

Figure S5. Cyclic Voltammogram of the $1 \mathbf{k}$ in $\mathrm{MeCN}$.

$\mathrm{E}_{\mathrm{p} / 2}\left(\mathbf{1 k} / \mathbf{1} \mathrm{k}^{\bullet-}\right)=-2.57 \mathrm{~V}$ vs $\mathrm{Fc}^{+} / \mathrm{Fc}$ in $\mathrm{MeCN}$. 


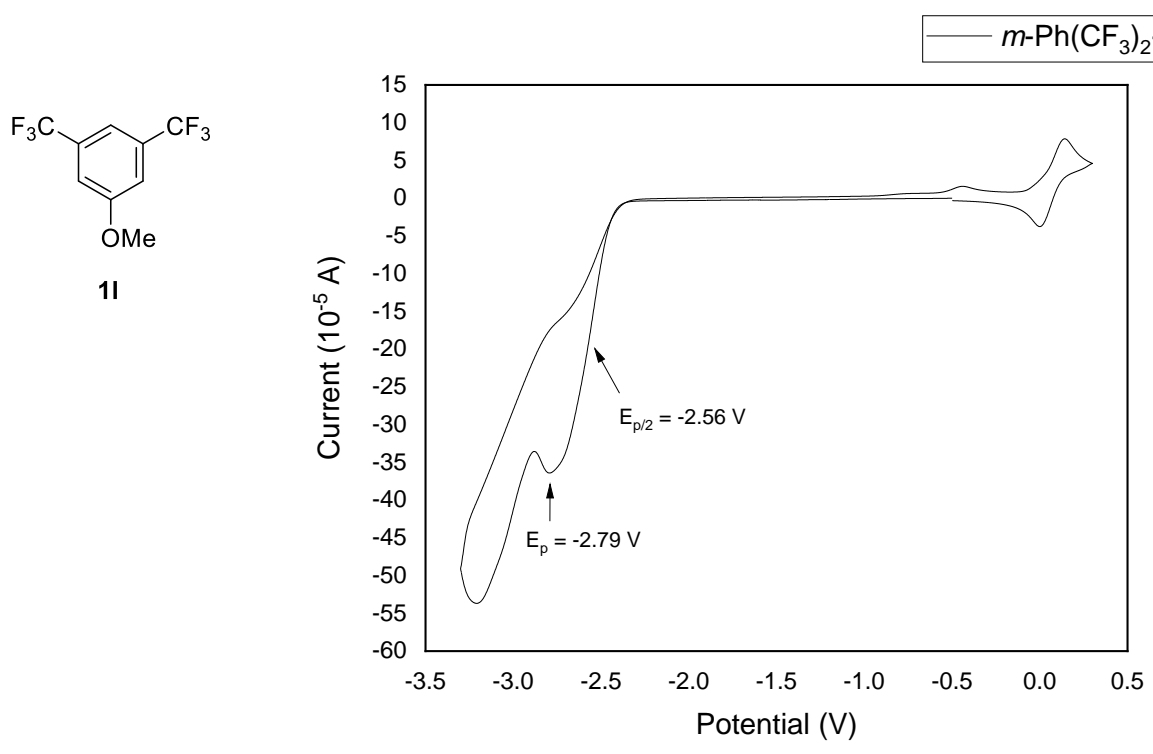

Figure S6. Cyclic Voltammogram of the $\mathbf{1 l}$ in MeCN.

$\mathrm{E}_{\mathrm{p} / 2}\left(\mathbf{1 1} / \mathbf{1 l ^ { \bullet } -}\right)=-2.64 \mathrm{~V} \mathrm{vs} \mathrm{Fc}^{+} / \mathrm{Fc}$ in $\mathrm{MeCN}$.

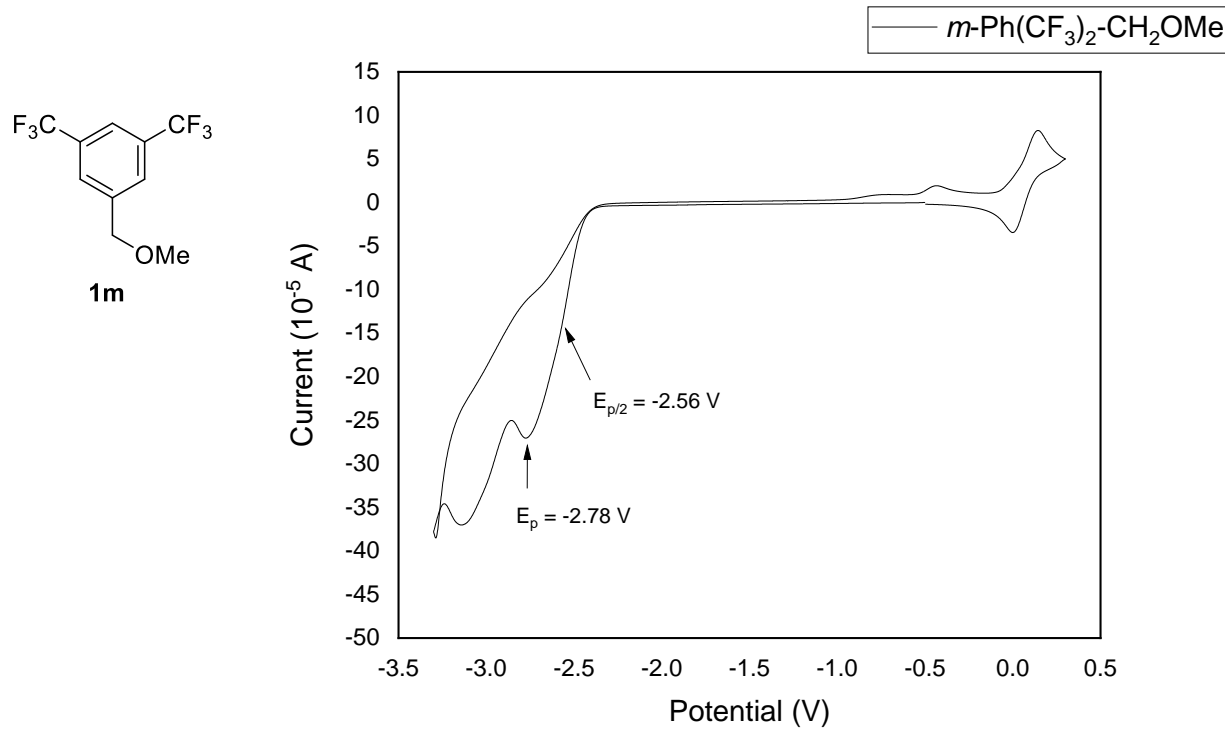

Figure S7. Cyclic Voltammogram of the $\mathbf{1 m}$ in $\mathrm{MeCN}$.

$\mathrm{E}_{\mathrm{p} / 2}\left(\mathbf{1 m} / \mathbf{1} \mathbf{m}^{\bullet-}\right)=-2.64 \mathrm{~V}$ vs $\mathrm{Fc}^{+} / \mathrm{Fc}$ in $\mathrm{MeCN}$. 


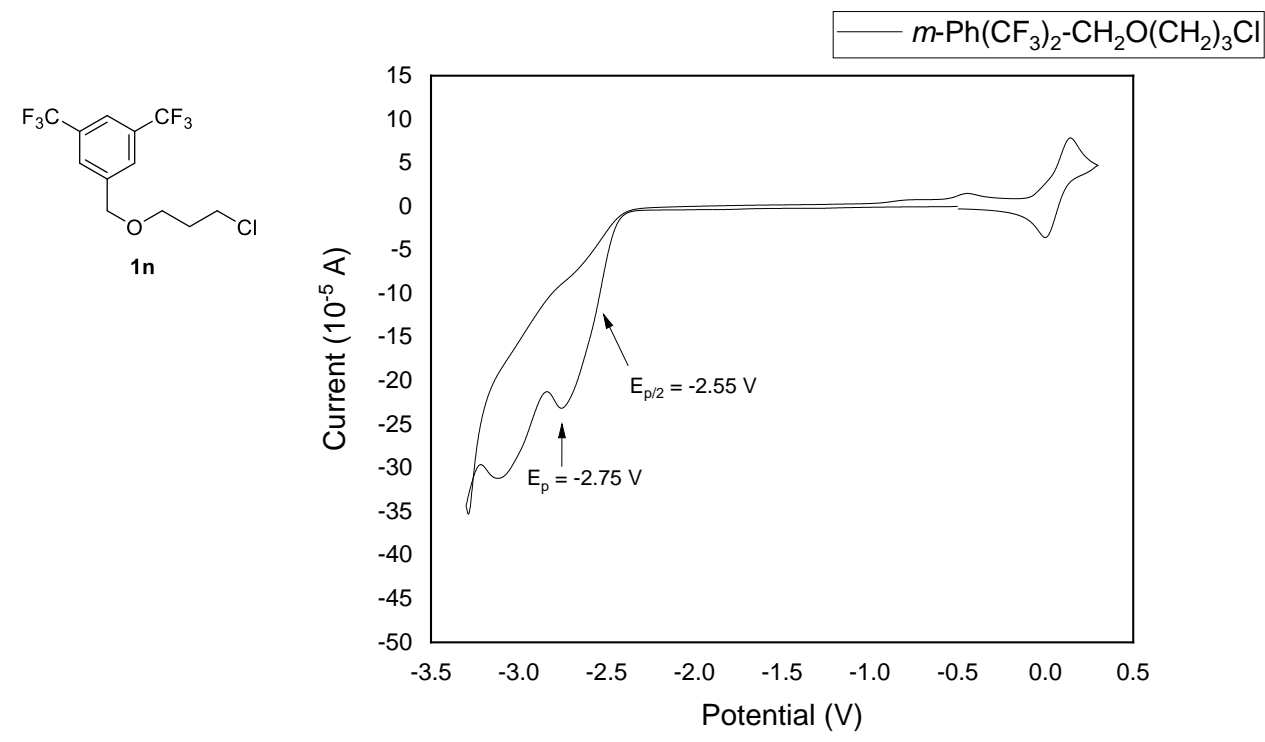

Figure S8. Cyclic Voltammogram of the 1n in $\mathrm{MeCN}$.

$\mathrm{E}_{\mathrm{p} / 2}\left(\mathbf{1 n} / \mathbf{1} \mathbf{n}^{\bullet}\right)=-2.62 \mathrm{~V}$ vs $\mathrm{Fc}^{+} / \mathrm{Fc}$ in $\mathrm{MeCN}$.
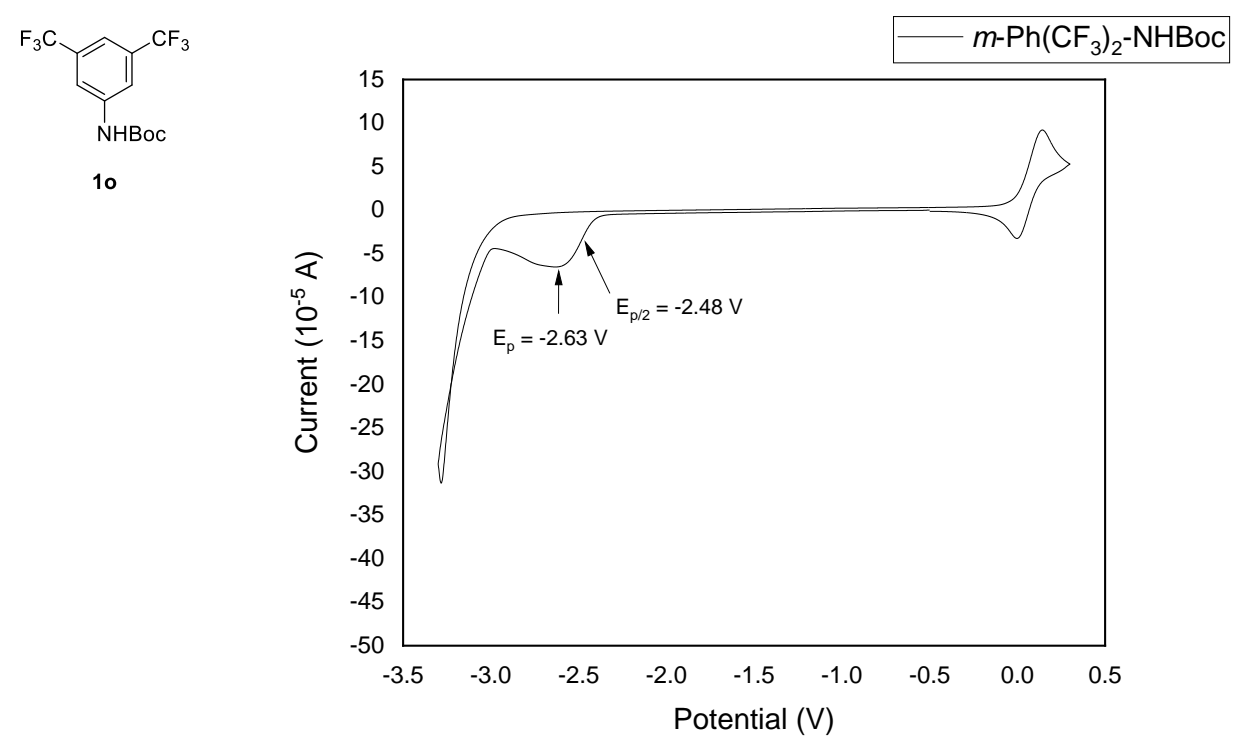

Figure S9. Cyclic Voltammogram of the 10 in $\mathrm{MeCN}$.

$\mathrm{E}_{\mathrm{p} / 2}\left(\mathbf{1 0} / \mathbf{1 0}{ }^{\bullet-}\right)=-2.55 \mathrm{~V} \mathrm{vs} \mathrm{Fc}^{+} / \mathrm{Fc}$ in $\mathrm{MeCN}$. 

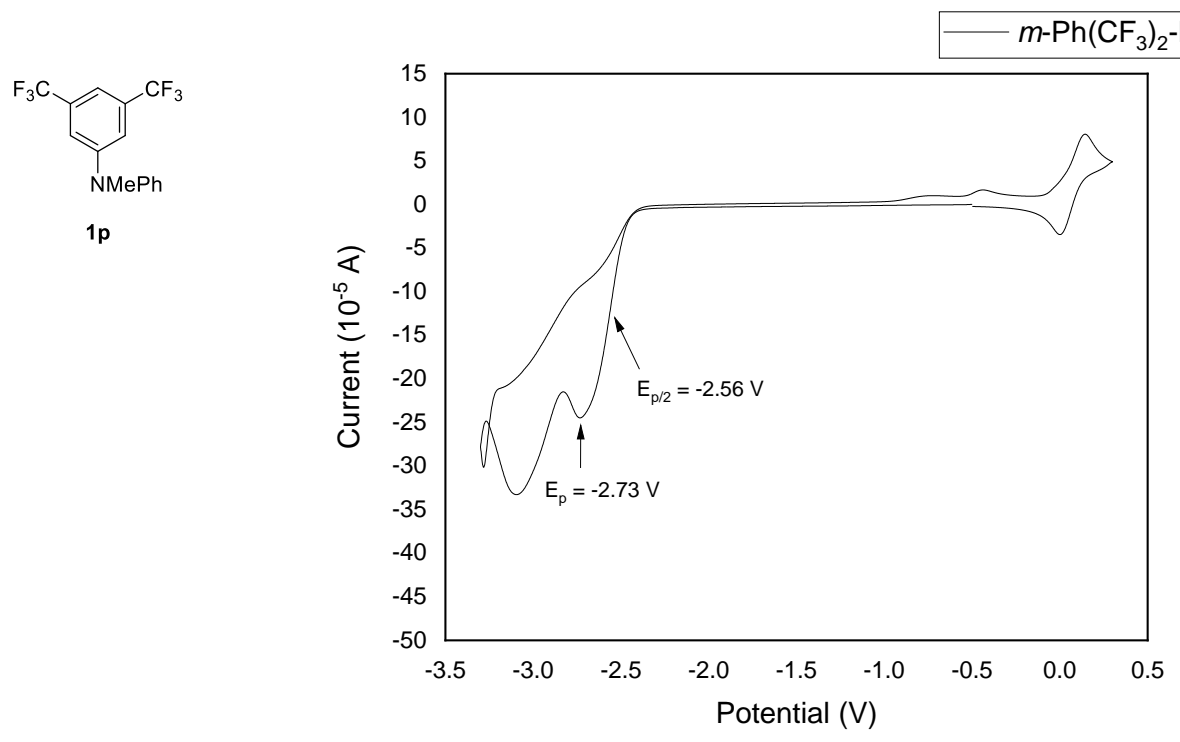

Figure S10. Cyclic Voltammogram of the 1p in MeCN.

$\mathrm{E}_{\mathrm{p} / 2}\left(\mathbf{1} \mathbf{p} / \mathbf{1} \mathbf{p}^{\bullet-}\right)=-2.63 \mathrm{~V}$ vs $\mathrm{Fc}^{+} / \mathrm{Fc}$ in $\mathrm{MeCN}$
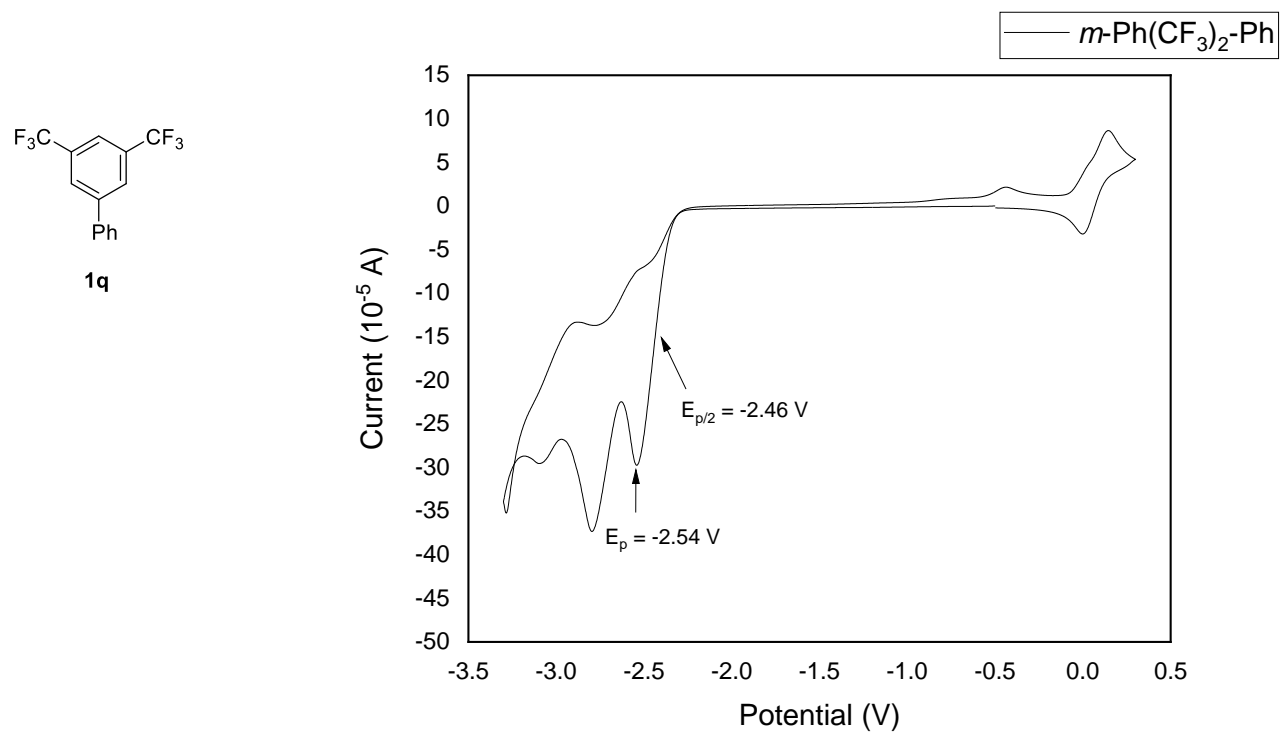

Figure S11. Cyclic Voltammogram of the 1q in $\mathrm{MeCN}$.

$\mathrm{E}_{\mathrm{p} / 2}\left(\mathbf{1} \mathbf{q} / \mathbf{1} \mathbf{q}^{\bullet-}\right)=-2.53 \mathrm{~V} \mathrm{vs} \mathrm{Fc}^{+} / \mathrm{Fc}$ in $\mathrm{MeCN}$ 

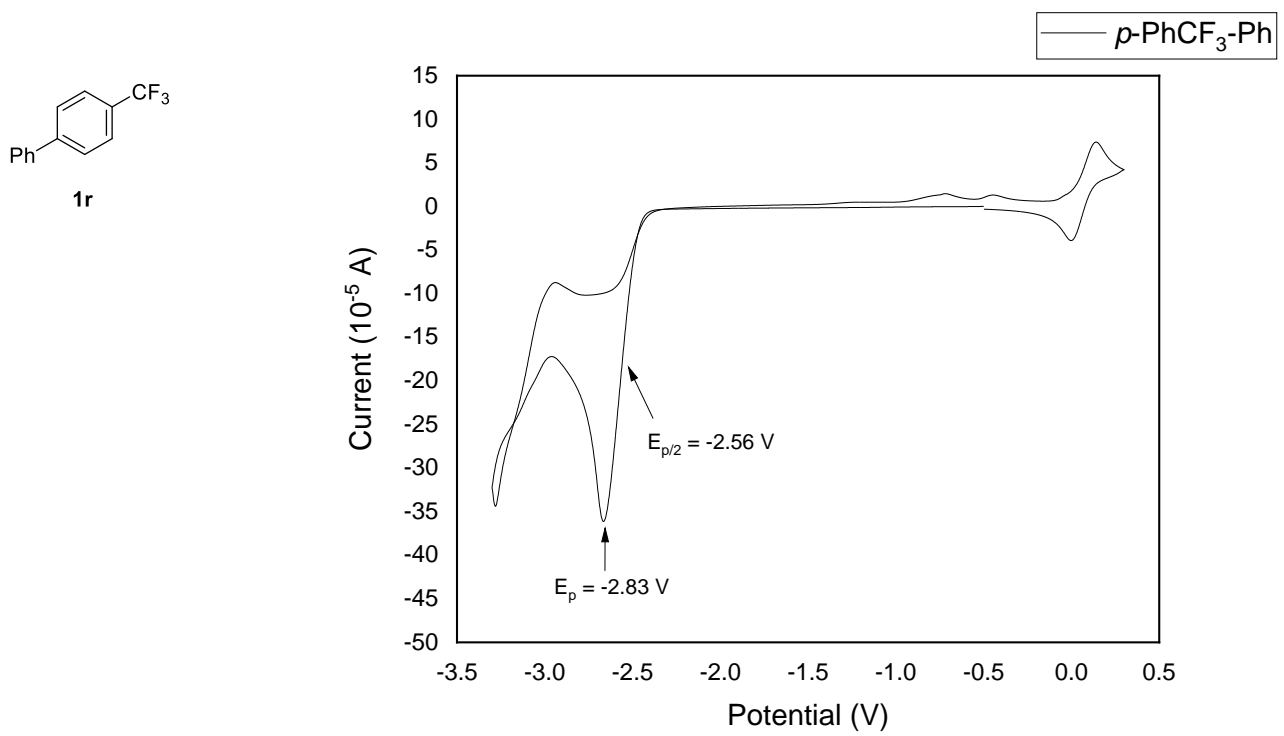

Figure S12. Cyclic Voltammogram of the 1r in MeCN.

$\mathrm{E}_{\mathrm{p} / 2}\left(\mathbf{1} \mathbf{r} / \mathbf{1} \mathbf{r}^{\bullet-}\right)=-2.63 \mathrm{~V}$ vs $\mathrm{Fc}^{+} / \mathrm{Fc}$ in $\mathrm{MeCN}$.
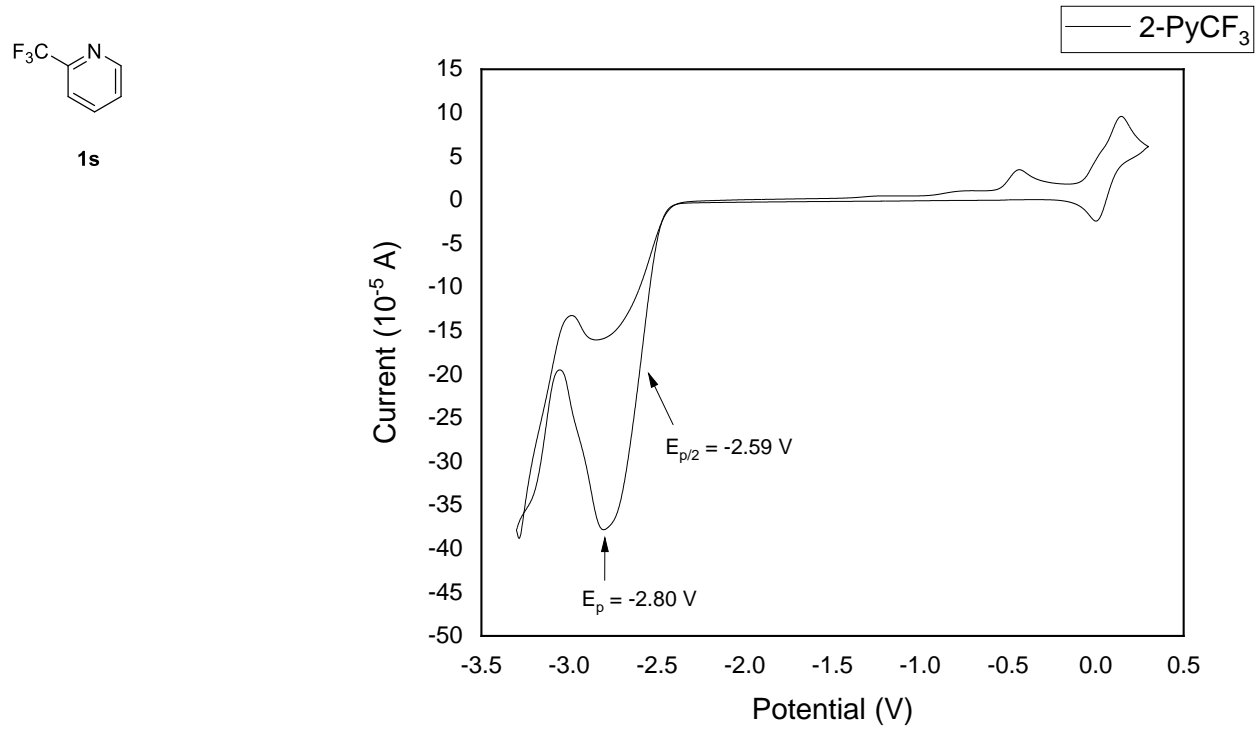

Figure S13. Cyclic Voltammogram of the 1s in MeCN.

$\mathrm{E}_{\mathrm{p} / 2}\left(\mathbf{1} \mathrm{s} / \mathbf{1} \mathrm{s}^{\bullet-}\right)=-2.67 \mathrm{~V}$ vs $\mathrm{Fc}^{+} / \mathrm{Fc}$ in $\mathrm{MeCN}$ 


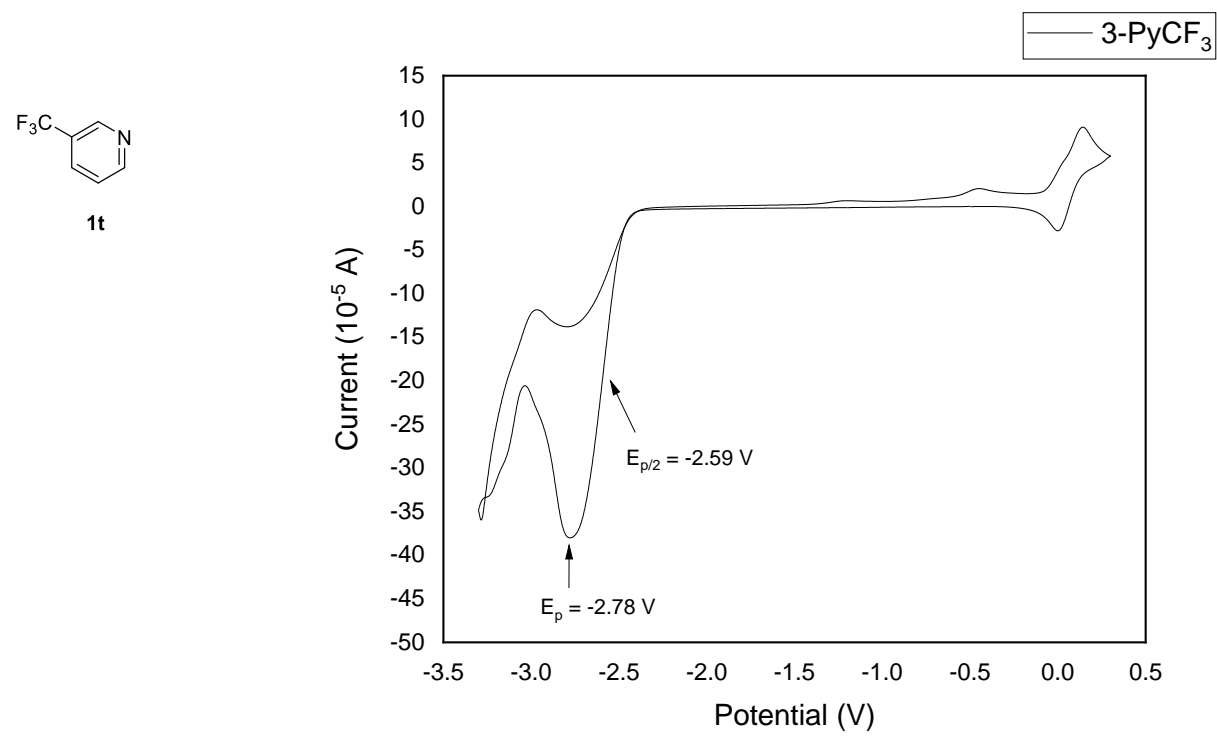

Figure S14. Cyclic Voltammogram of the 1t in $\mathrm{MeCN}$.

$\mathrm{E}_{\mathrm{p} / 2}\left(\mathbf{1 t} / \mathbf{1 t ^ { \bullet - }}\right)=-2.66 \mathrm{~V}$ vs $\mathrm{Fc}^{+} / \mathrm{Fc}$ in $\mathrm{MeCN}$
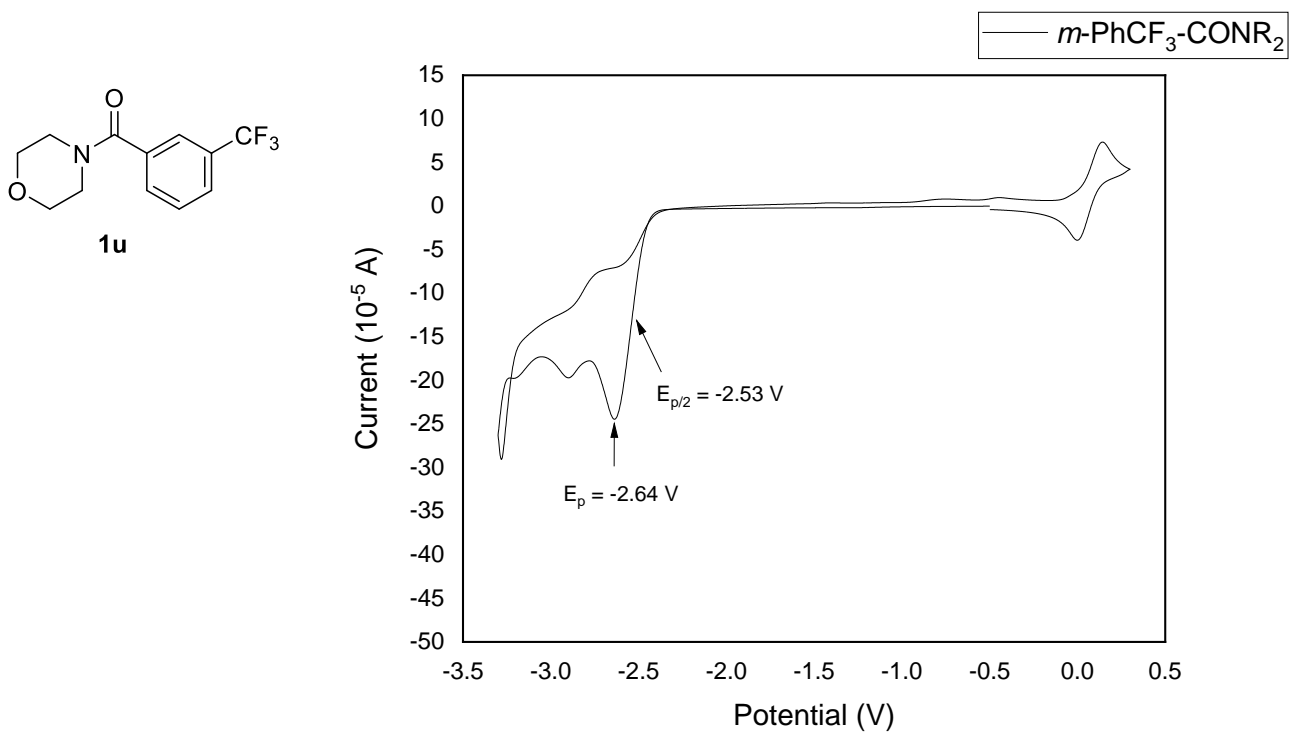

Figure S13. Cyclic Voltammogram of the 1u in MeCN.

$\mathrm{E}_{\mathrm{p} / 2}\left(\mathbf{1} \mathbf{u} / \mathbf{1} \mathbf{u}^{\bullet-}\right)=-2.60 \mathrm{~V}$ vs $\mathrm{Fc}^{+} / \mathrm{Fc}$ in $\mathrm{MeCN}$. 

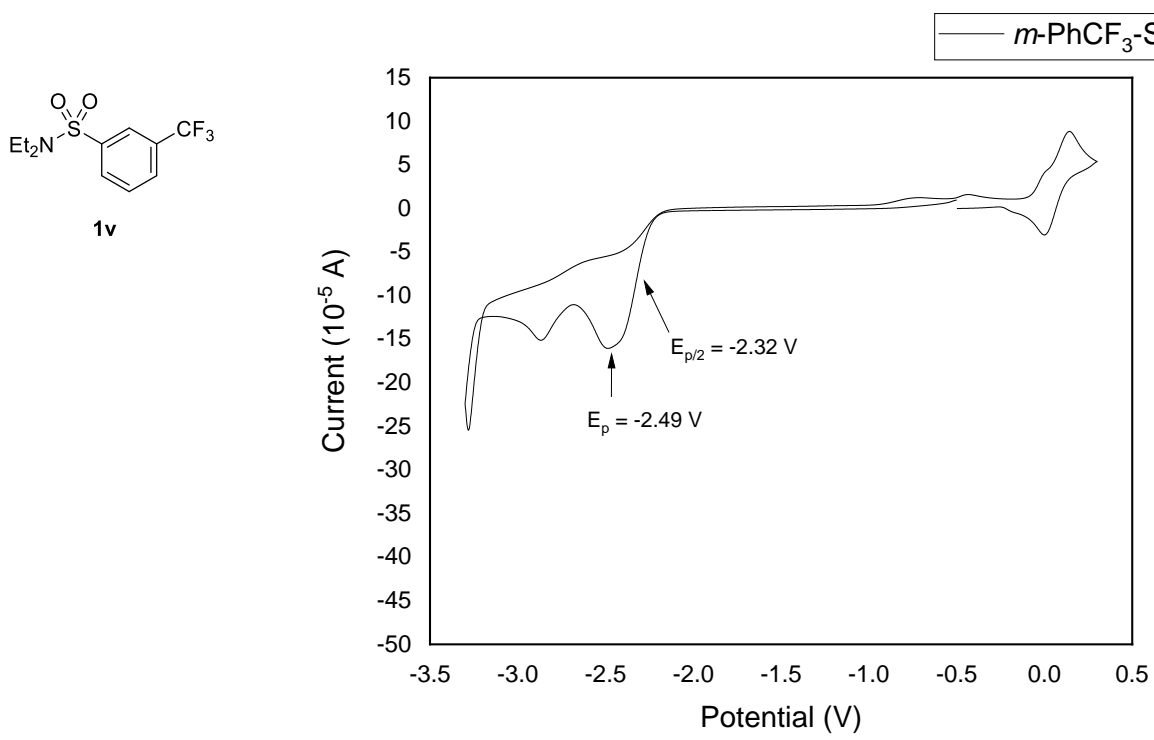

Figure S14. Cyclic Voltammogram of the $1 \mathbf{v}$ in MeCN.

$\mathrm{E}_{\mathrm{p} / 2}\left(\mathbf{1 v} / \mathbf{1} \mathbf{v}^{\bullet-}\right)=-2.39 \mathrm{~V} \mathrm{vs} \mathrm{Fc}^{+} / \mathrm{Fc}$ in $\mathrm{MeCN}$.<smiles>N#Cc1cccc(C(F)(F)F)c1</smiles>

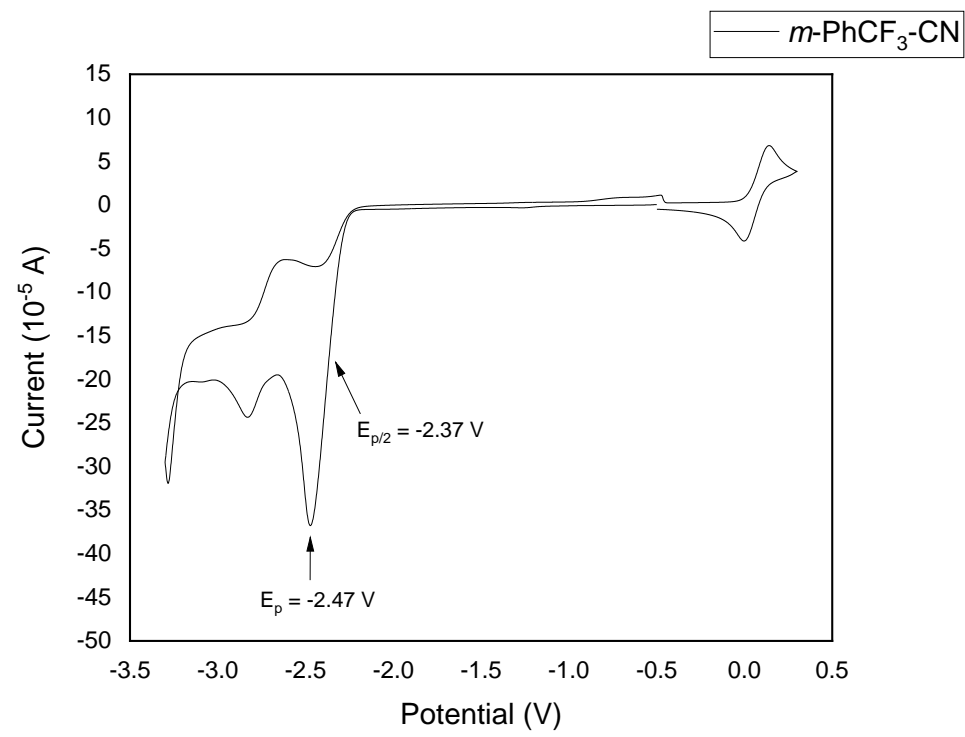

Figure S15. Cyclic Voltammogram of the $1 w$ in $\mathrm{MeCN}$.

$\mathrm{E}_{\mathrm{p} / 2}\left(\mathbf{1} \mathbf{w} / \mathbf{1} \mathbf{w}^{\bullet-}\right)=-2.44 \mathrm{~V}$ vs $\mathrm{Fc}^{+} / \mathrm{Fc}$ in $\mathrm{MeCN}$ 

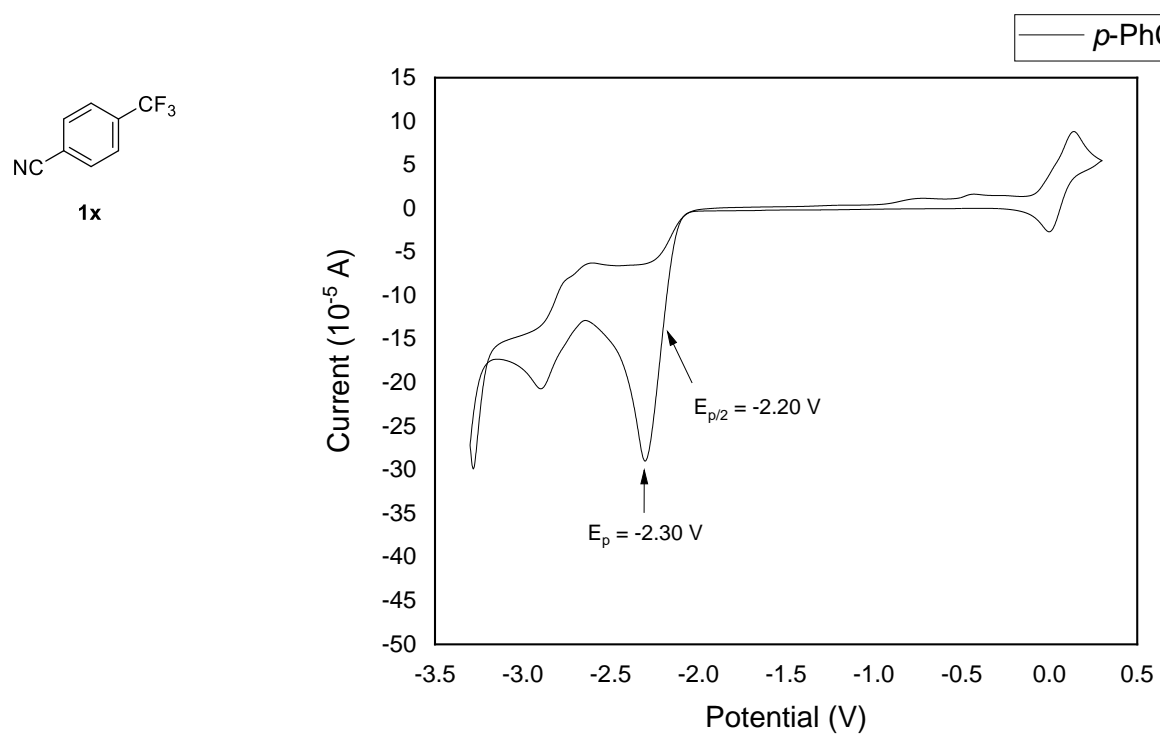

Figure S16. Cyclic Voltammogram of the $1 x$ in MeCN.

$\mathrm{E}_{\mathrm{p} / 2}\left(\mathbf{1} \mathbf{x} / \mathbf{1} \mathbf{x}^{\bullet-}\right)=-2.27 \mathrm{~V}$ vs $\mathrm{Fc}^{+} / \mathrm{Fc}$ in $\mathrm{MeCN}$.
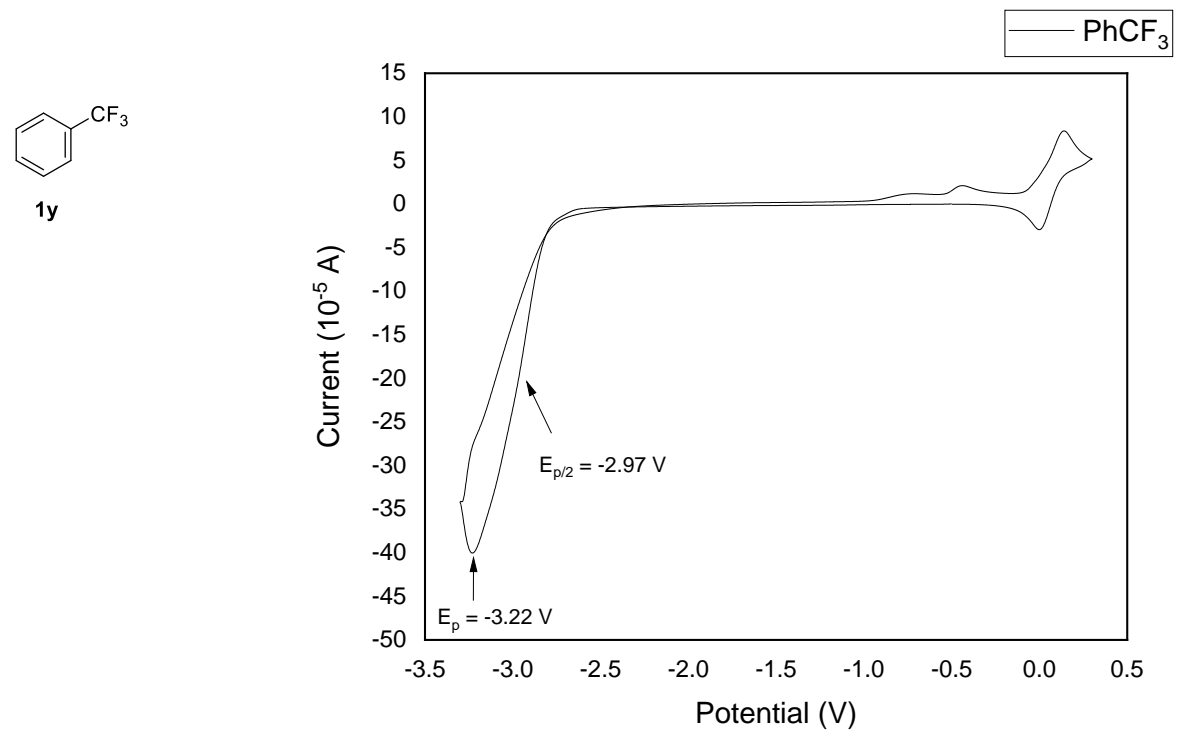

Figure S17. Cyclic Voltammogram of the $\mathbf{1 y}$ in MeCN.

$\mathrm{E}_{\mathrm{p} / 2}\left(\mathbf{1 y} / \mathbf{1} \mathbf{y}^{\bullet-}\right)=-3.04 \mathrm{~V} \mathrm{vs} \mathrm{Fc}^{+} / \mathrm{Fc}$ in $\mathrm{MeCN}$. 


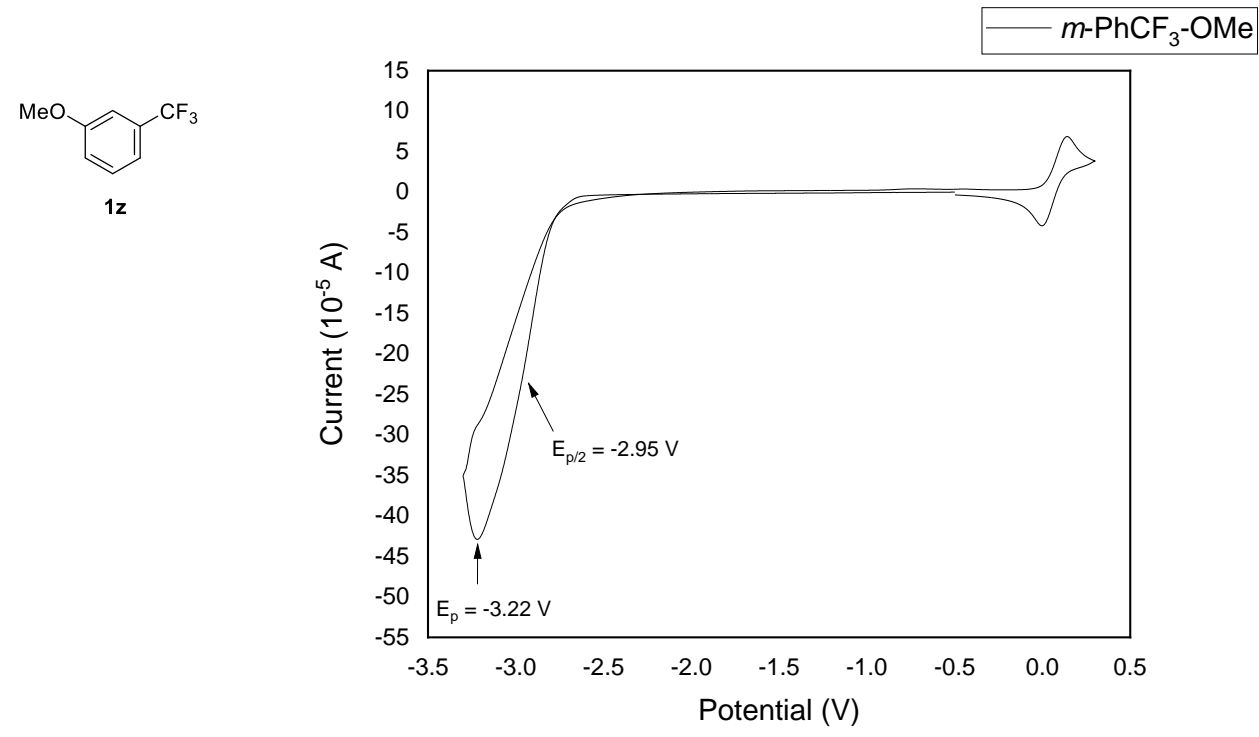

Figure S18. Cyclic Voltammogram of the $\mathbf{1 z}$ in $\mathrm{MeCN}$.

$\mathrm{E}_{\mathrm{p} / 2}\left(\mathbf{1 z} / \mathbf{1 z} \mathbf{z}^{--}\right)=-3.02 \mathrm{~V} \mathrm{vs} \mathrm{Fc}^{+} / \mathrm{Fc}$ in $\mathrm{MeCN}$.

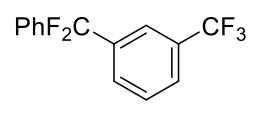

3a

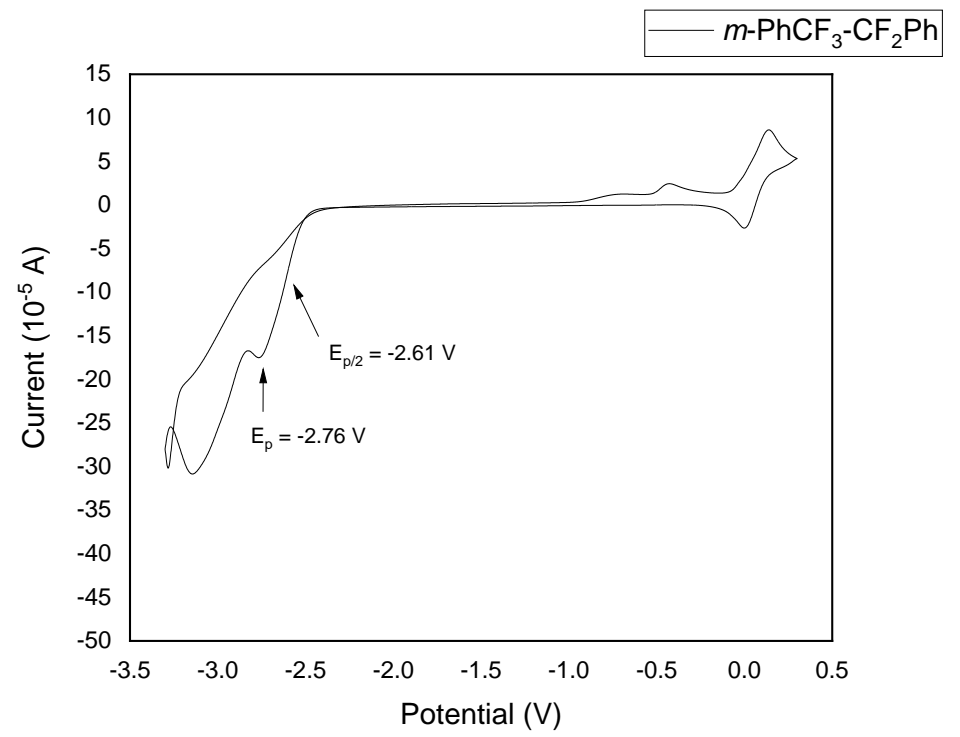

Figure S19. Cyclic Voltammogram of the 3a in MeCN.

$\mathrm{E}_{\mathrm{p} / 2}\left(\mathbf{3 a} / \mathbf{3 a} \mathbf{a}^{\bullet-}\right)=-2.68 \mathrm{~V}$ vs $\mathrm{Fc}^{+} / \mathrm{Fc}$ in $\mathrm{MeCN}$ 
baseline:

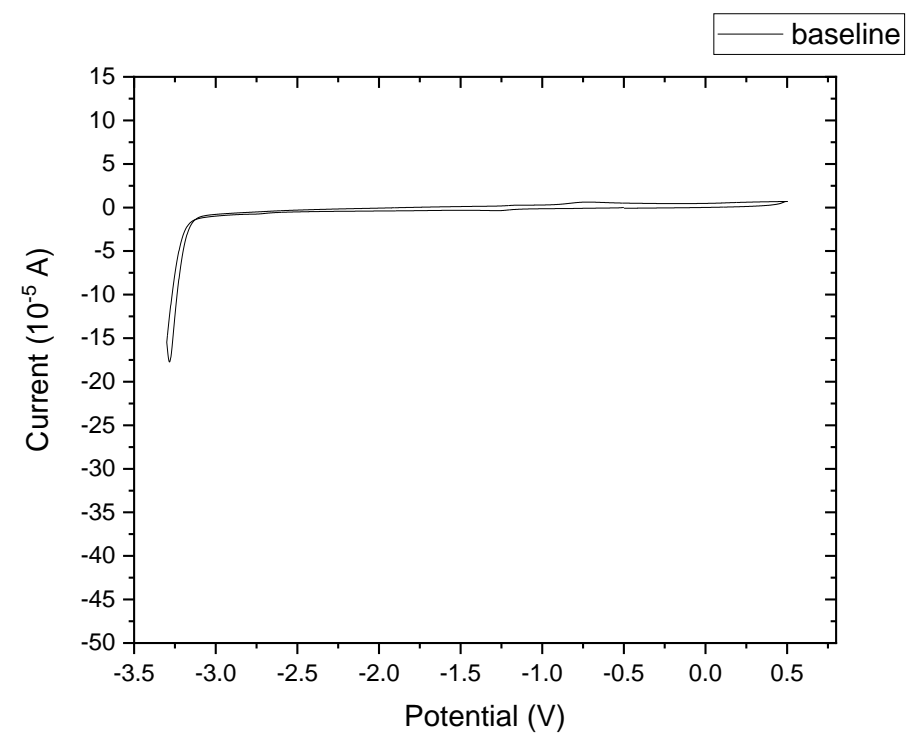

Figure S20. baseline in the absence of substrate. 


\section{$5.2{ }^{31} \mathrm{P}$ NMR monitoring experiments of the resting state of palladium catalyst}

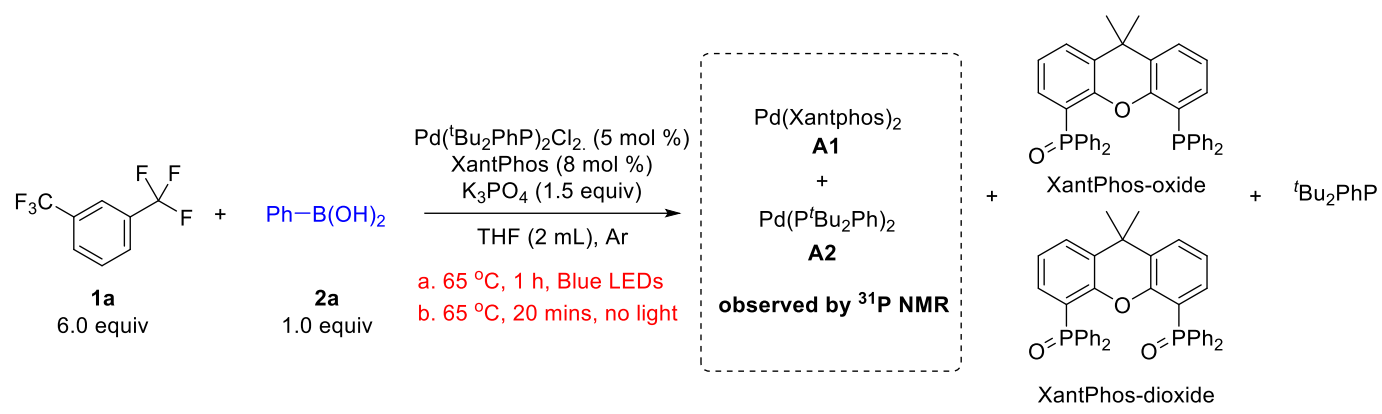

a. after irradiation

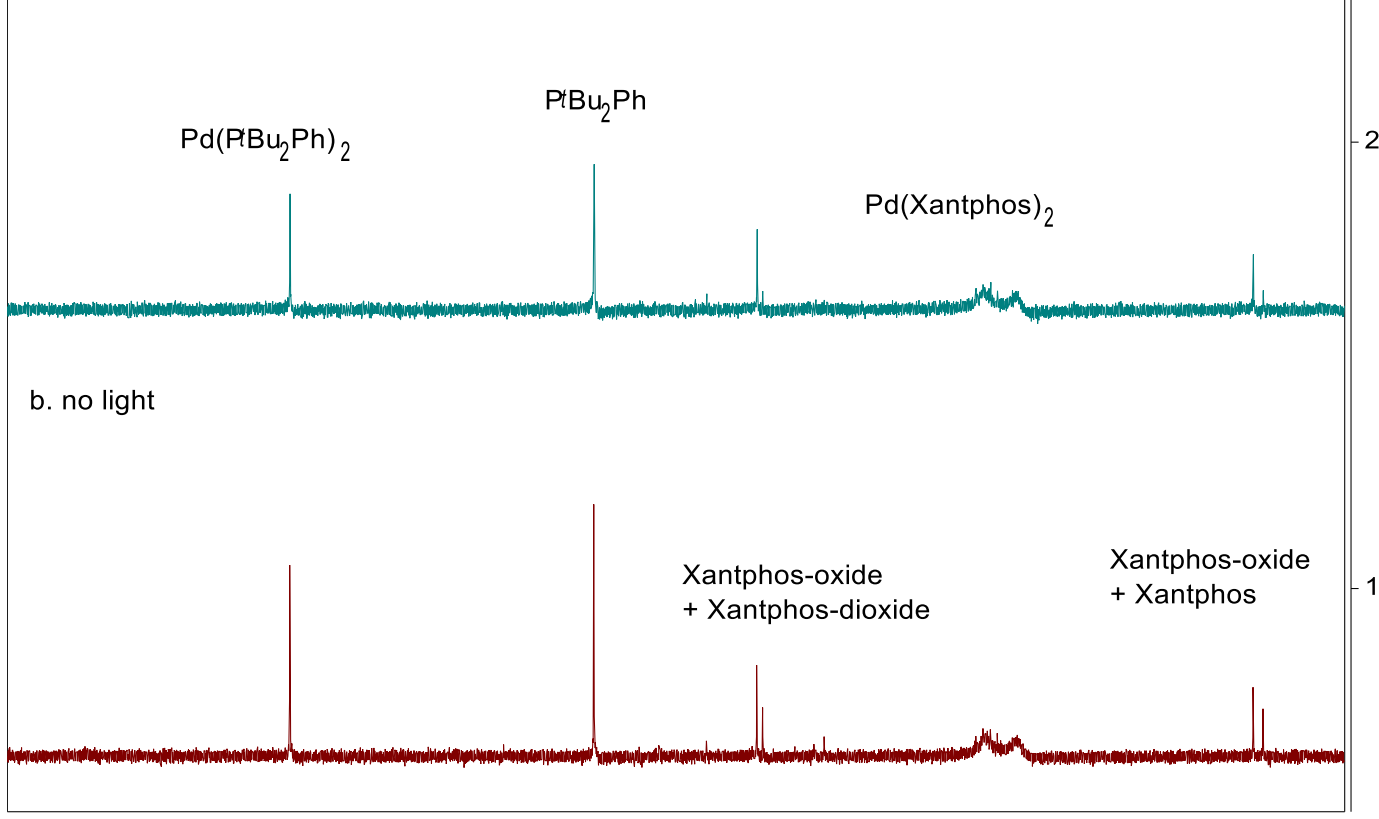

$\begin{array}{llllllllllllllllllllllllll}90 & 85 & 80 & 75 & 70 & 65 & 60 & 55 & 50 & 45 & 40 & 35 & 30 & 25 & 20 & 15 & 10 & 5 & 0 & -5 & -10 & -15 & -20 & -25 & -30 \\ \mathrm{f} 1(\mathrm{ppm})\end{array}$

Figure S21. ${ }^{31}$ P NMR sepectra of the reaction mixture: a) after irradiation of the reaction with blue light for $1 \mathrm{~h}$; b) after the reaction was stirred for $20 \mathrm{~min}$ without irradiation of blue light

Procedure: To a $25 \mathrm{~mL}$ of Schlenk tube were added $\mathrm{Pd}\left({ }^{t} \mathrm{Bu}_{2} \mathrm{PhP}_{2}{ }_{2} \mathrm{Cl}_{2}(9.3 \mathrm{mg}, 0.015 \mathrm{mmol}, 5 \mathrm{~mol} \%)\right.$, Xantphos (13.9 mg, $0.024 \mathrm{mmol}, 8 \mathrm{~mol} \%)$, and $\mathrm{PhB}(\mathrm{OH})_{2} \mathbf{2 a}\left(0.3 \mathrm{mmol}, 1.0\right.$ equiv) in the air. $\mathrm{K}_{3} \mathrm{PO}_{4}$ ( $95.5 \mathrm{mg}, 0.45 \mathrm{mmol}, 1.5$ equiv) was added in the glovebox. The reaction mixture was then evacuated and backfilled with $\operatorname{Ar}(3$ times). 1a $(280 \mu \mathrm{L}, 1.8 \mathrm{mmol}, 6.0$ equiv) and THF (2.0 mL) were then added. The reaction mixture was stirred under irradiation of blue LEDs strip $(12 \mathrm{~W} \times 2)$ for $1 \mathrm{~h}$ at $65{ }^{\circ} \mathrm{C}$ (condition a) or stirred at $65^{\circ} \mathrm{C}$ for $20 \mathrm{~min}$ in the absence of blue light (condition b). Then the reaction mixture was cooled to room temperature and analyzed by ${ }^{31} \mathrm{P}$ NMR. 

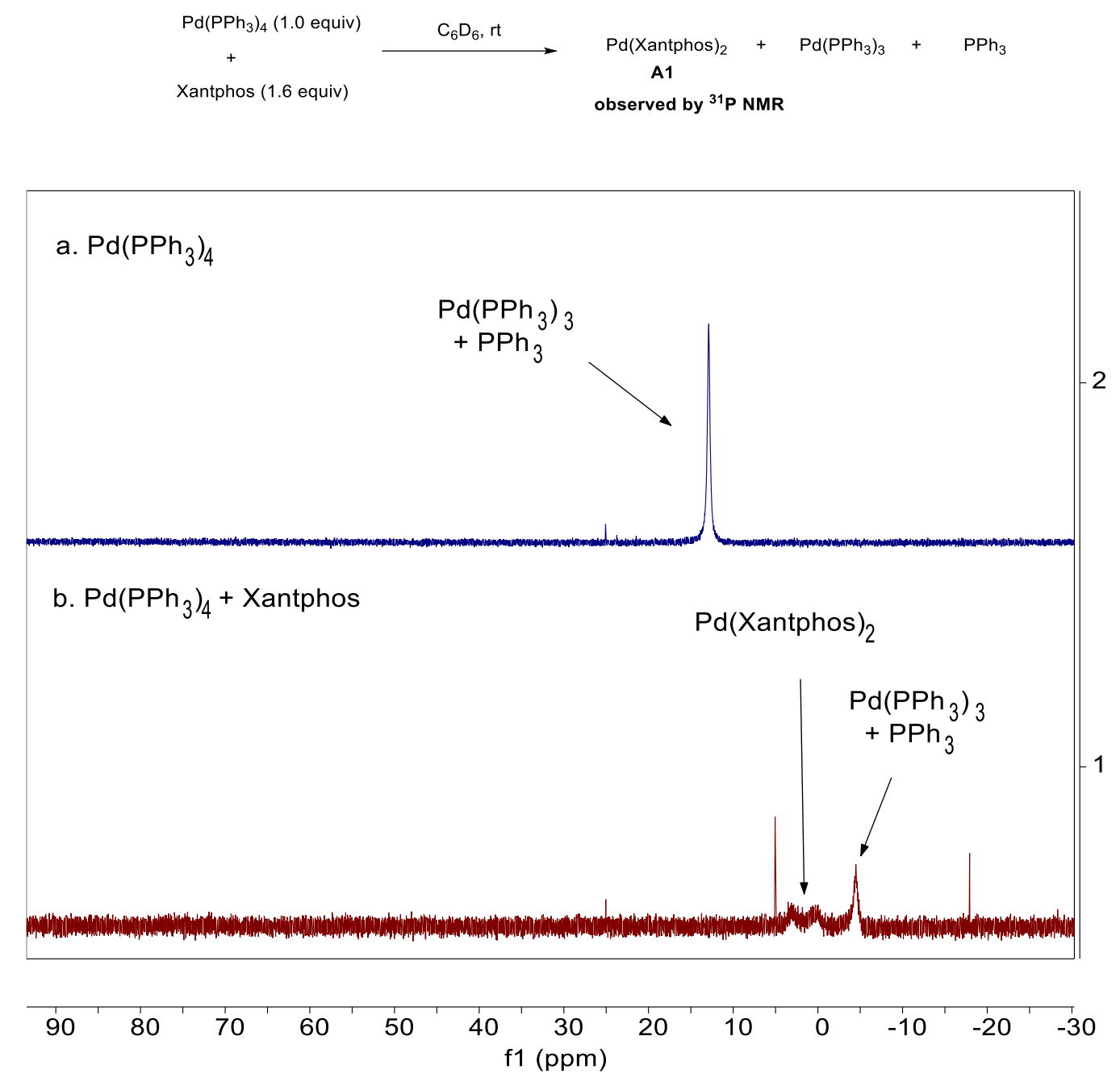

Figure S22. ${ }^{31} \mathrm{P}$ NMR sepectra of the mixture: a) $\mathrm{Pd}\left(\mathrm{PPh}_{3}\right)_{4}$; b) $\mathrm{Pd}\left(\mathrm{PPh}_{3}\right)_{4}$ and $\mathrm{Xantphos}$ in $\mathrm{C}_{6} \mathrm{D}_{6}$.

Procedure: To a NMR tube were added $\mathrm{Pd}\left(\mathrm{PPh}_{3}\right)_{4}(2.3 \mathrm{mg}, 2 \mu \mathrm{mol}, 1.0$ equiv), Xantphos (1.9 mg, $3.2 \mu \mathrm{mol}, 1.6$ equiv) and $\mathrm{C}_{6} \mathrm{D}_{6}(0.75 \mathrm{~mL})$ in the glovebox. Then the reaction mixture was shaked for $5 \mathrm{~min}$ at room temperature and analyzed by ${ }^{31} \mathrm{P} \mathrm{NMR}$. 


\subsection{UV-vis absorption spectra of the reaction mixture in THF}

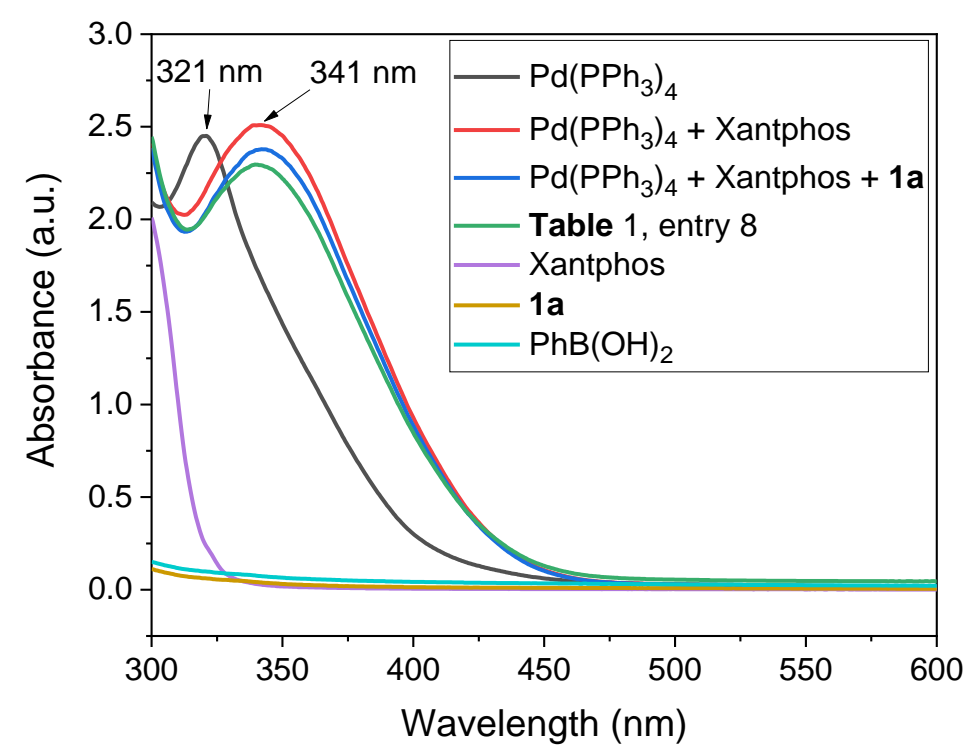

Figure S23. UV-vis absorption spectra of reaction mixture. The concentration of each component is $1 / 75$ of the reaction conditions of Table 1 , entry 8 . $[\mathrm{Pd}]=0.1 \mathrm{mM}$. 
5.4 Emission spectra of $\operatorname{Pd}(0)$ species

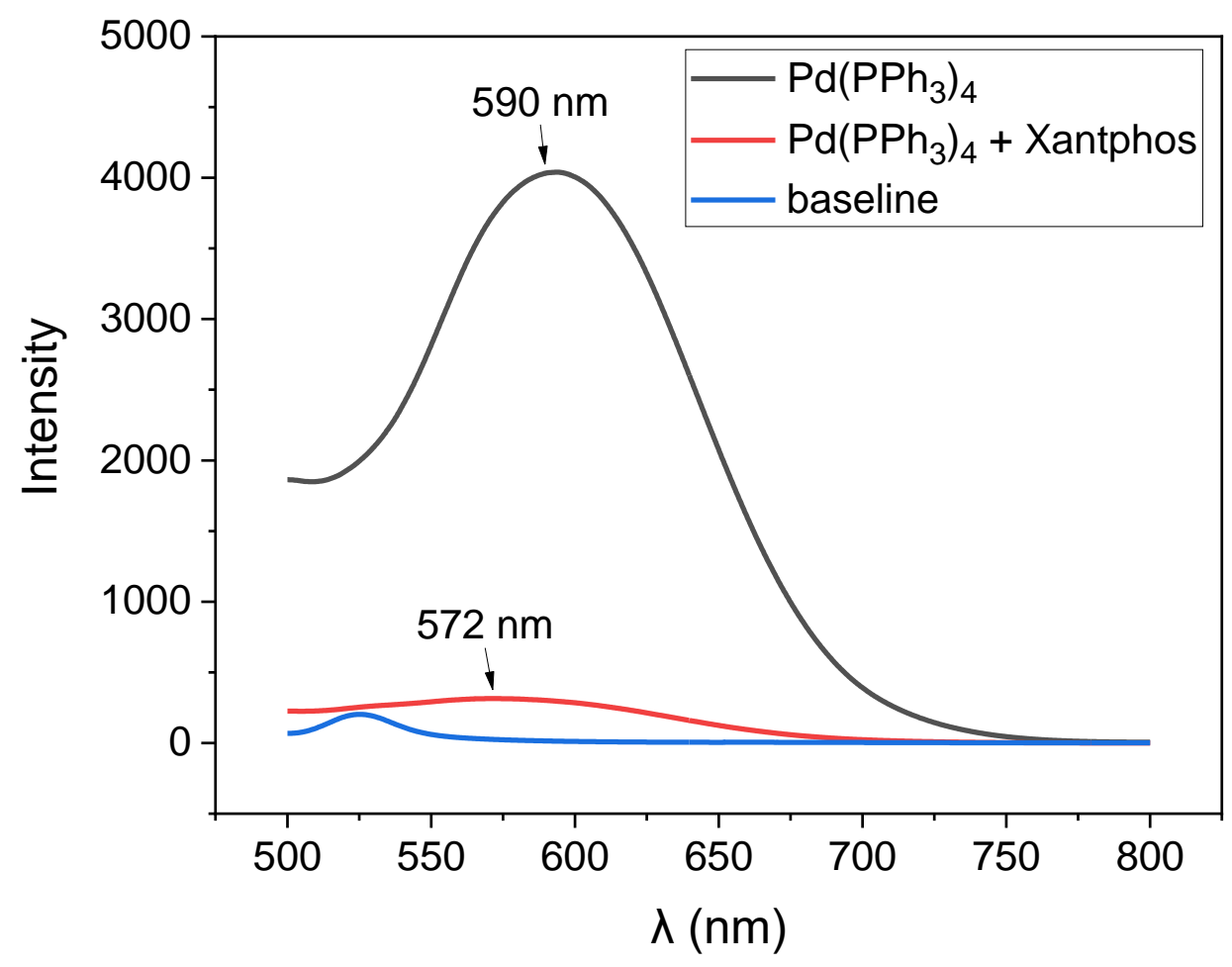

Figure S24. Emission spectra of palladium catalyst after irradiation at $450 \mathrm{~nm}$. $[\mathrm{Pd}]=2.5 \mathrm{mM}$ in THF. 
5.5 Luminescence quenching of $\operatorname{Pd}\left(\mathrm{PPh}_{3}\right)_{4}$ by $1 \mathrm{a}$
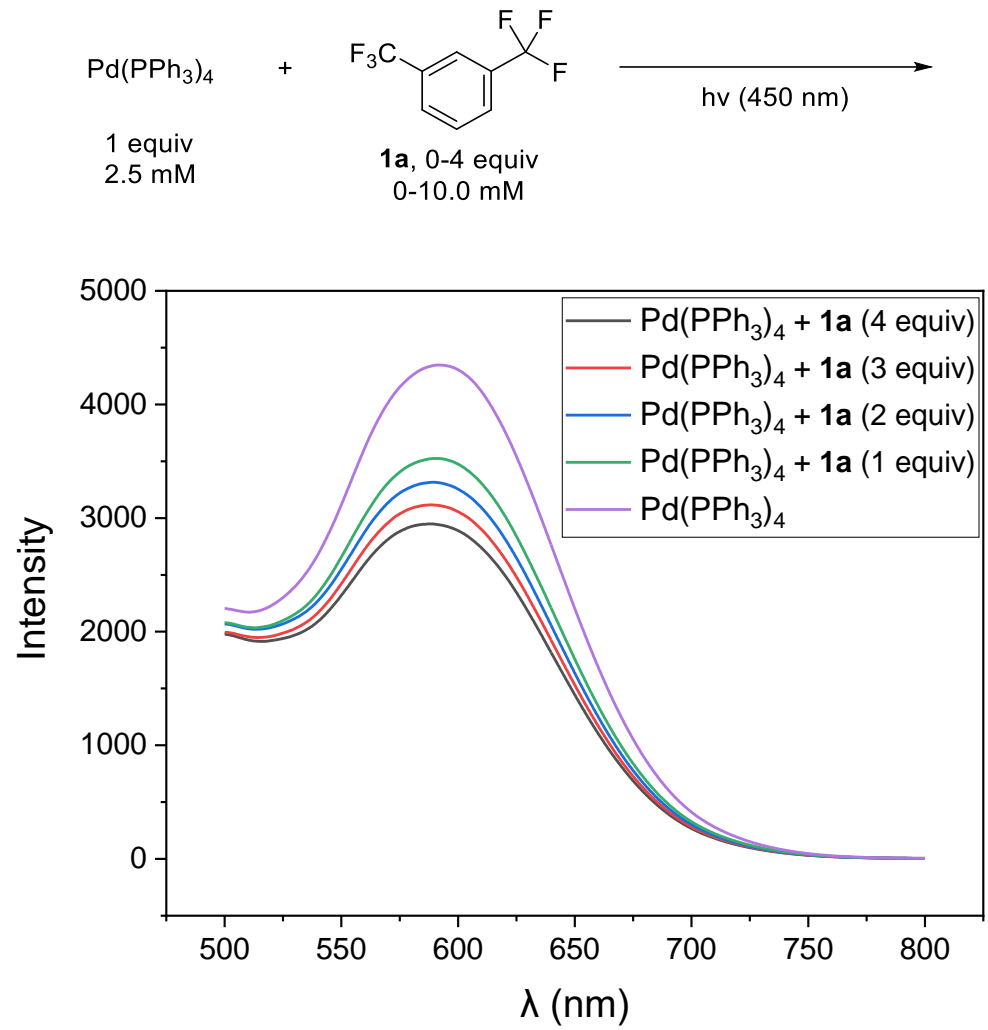

Figure S25. Emission quenching of $\mathrm{Pd}\left(\mathrm{PPh}_{3}\right)_{4}$ by $\mathbf{1 a}$ after irradiation at $450 \mathrm{~nm}$. [Pd] $=2.5 \mathrm{mM}$ in THF.

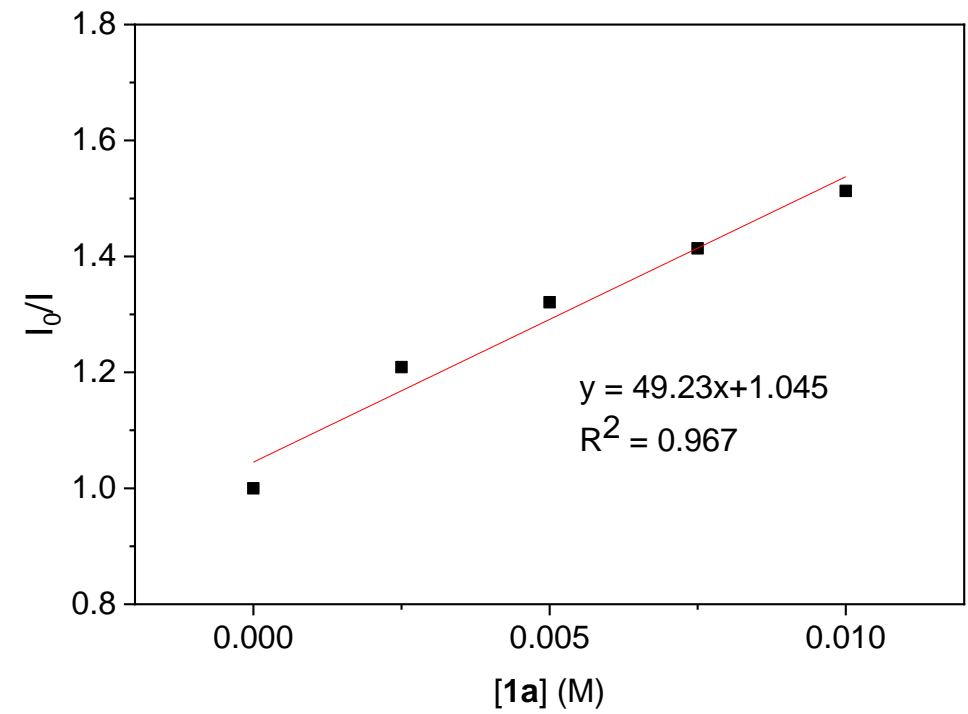

Figure S26. Stern-Volmer plot for the emission quenching of $\mathrm{Pd}\left(\mathrm{PPh}_{3}\right)_{4}$ by 1 a. 


\subsection{Calculation of oxidation potential of excited palladium catalyst}

The zero-zero vibrational state excitation energy $E_{0,0}$ was estimated by the corresponding energy of the $\lambda_{\max }$ in emission spectra $\left(\mathrm{E}_{0,0}=\mathrm{hc} / \lambda\right)$. Excited state oxidation potentials were calculated by the following approximating formulas:

$$
\mathrm{E}_{1 / 2}{ }^{\mathrm{ox}}(\mathrm{PC})=\mathrm{E}_{1 / 2}\left(\mathrm{PC}^{\bullet+} / \mathrm{PC}^{*}\right)=\mathrm{E}_{1 / 2}\left(\mathrm{PC}^{\bullet+} / \mathrm{PC}\right)-\mathrm{E}_{0,0} .
$$

Table S8. Oxidation potential of excited palladium catalyst

\begin{tabular}{|c|c|c|c|}
\hline Palladium catalyst & $\begin{array}{c}\mathrm{E}_{1 / 2}\left(\left[\mathrm{Pd}^{\mathrm{I}}\right] /\left[\mathrm{Pd}^{0}\right]\right) \\
\text { vs. } \mathrm{Fc}^{+} / \mathrm{Fc} \text { in } \mathrm{THF}\end{array}$ & $\lambda_{\max }$ & $\begin{array}{l}\mathrm{E}_{1 / 2}\left(\left[\mathrm{Pd}^{\mathrm{I}}\right] /\left[\mathrm{Pd}^{0}\right]^{*}\right) \\
\text { vs. } \mathrm{Fc}^{+} / \mathrm{Fc} \text { in THF }\end{array}$ \\
\hline $\mathrm{Pd}\left(\mathrm{PPh}_{3}\right)_{4}$ & $-0.41 \mathrm{~V}$ & $590 \mathrm{~nm}$ & $-2.51 \mathrm{~V}$ \\
\hline $\operatorname{Pd}(\text { Xantphos })_{2}$ & $-0.59 \mathrm{~V}$ & $572 \mathrm{~nm}$ & $-2.76 \mathrm{~V}$ \\
\hline
\end{tabular}




\subsection{Radical clock experiment}

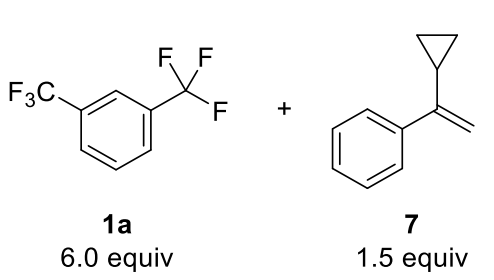

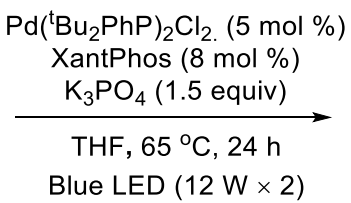

$\mathrm{Ph}-\mathrm{B}(\mathrm{OH})_{2} \quad \mathbf{2 a}(1.0$ equiv $)$

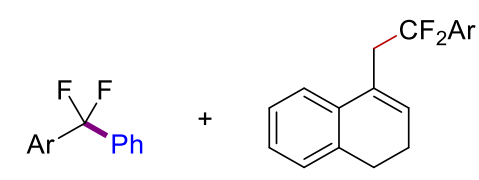

$3 \mathbf{a}$
$(12 \%)$
8

$39 \%(25 \%)$

Procedure: To a $25 \mathrm{~mL}$ of Schlenk tube were added $\mathrm{Pd}\left({ }^{t} \mathrm{Bu}_{2} \mathrm{PhP}\right)_{2} \mathrm{Cl}_{2}(9.3 \mathrm{mg}, 0.015 \mathrm{mmol}, 5 \mathrm{~mol} \%)$, Xantphos (13.9 mg, $0.024 \mathrm{mmol}, 8 \mathrm{~mol} \%)$, and $\mathrm{PhB}(\mathrm{OH})_{2} \mathbf{2 a}\left(0.3 \mathrm{mmol}, 1.0\right.$ equiv) in the air. $\mathrm{K}_{3} \mathrm{PO}_{4}$ ( $95.5 \mathrm{mg}, 0.45 \mathrm{mmol}, 1.5$ equiv) was added in the glovebox. The reaction mixture was then evacuated and backfilled with $\mathrm{Ar}$ (3 times). $\mathrm{ArCF}_{3} \mathbf{1 a}(280 \mu \mathrm{L}, 1.8 \mathrm{mmol}, 6$ equiv), alkene 7 (64.9 mg, $0.45 \mathrm{mmol}$, 1.5 equiv) and THF (2.0 mL) were added. After pre-stirring at $65^{\circ} \mathrm{C}$ for $20 \mathrm{~min}$, the reaction mixture was stirred and irradiated for $24 \mathrm{~h}$ at $65{ }^{\circ} \mathrm{C}$ under blue LEDs strips $(12 \mathrm{~W} \times 2)$. The reaction mixture was cooled to room temperature and the yield was determined by ${ }^{19} \mathrm{~F}$ NMR using fluorobenzene as an internal standard. The yield of compound 3a was based on $\mathbf{2 a}$, and the yield of compound $\mathbf{8}$ was based on 7. The resulting mixture was filtered with a pad of celite. The filtrate was concentrated and the residue was purified with flash chromatography or preparative HPLC to afford the title compounds.

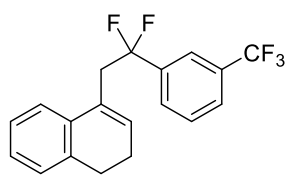

4-(2,2-Difluoro-2-(3-(trifluoromethyl)phenyl)ethyl)-1,2-

dihydronaphthalene (8). The product (39\% yield determined by ${ }^{19}$ F NMR; 37.8 $\mathrm{mg}, 25 \%$ yield) was purified with reverse-phase preparative $\mathrm{HPLC}\left(\mathrm{CH}_{3} \mathrm{CN}\right.$ :

$\left.\mathrm{H}_{2} \mathrm{O}=8: 2\right)$ as a colorless oil. ${ }^{1} \mathbf{H}$ NMR $\left(400 \mathrm{MHz} \mathrm{CDCl}_{3}\right) \delta 7.63(\mathrm{~s}, 1 \mathrm{H}), 7.62-7.56(\mathrm{~m}, 2 \mathrm{H}), 7.46$ $(\mathrm{dd}, J=7.8,7.8 \mathrm{~Hz}, 1 \mathrm{H}), 7.13-7.07(\mathrm{~m}, 4 \mathrm{H}), 5.83(\mathrm{t}, J=4.4 \mathrm{~Hz}, 1 \mathrm{H}), 3.29(\mathrm{t}, J=15.1 \mathrm{~Hz}, 2 \mathrm{H}), 2.68$ $(\mathrm{t}, J=8.0 \mathrm{~Hz}, 2 \mathrm{H}), 2.23-2.15(\mathrm{~m}, 2 \mathrm{H}) .{ }^{19} \mathbf{F} \mathbf{N M R}\left(376 \mathrm{MHz}, \mathrm{CDCl}_{3}\right) \delta-62.84(\mathrm{~s}, 3 \mathrm{~F}),-93.75(\mathrm{t}, J=$ $15.1 \mathrm{~Hz}, 2 \mathrm{~F}) .{ }^{13} \mathrm{C}$ NMR $\left(126 \mathrm{MHz}, \mathrm{CDCl}_{3}\right) \delta 137.9$ (t, $\left.J=27.5 \mathrm{~Hz}\right), 136.2,134.0,132.0,130.5(\mathrm{q}, J$ $=32.7 \mathrm{~Hz}), 128.7,128.6(\mathrm{t}, J=5.8 \mathrm{~Hz}), 128.5(\mathrm{t}, J=4.5 \mathrm{~Hz}), 127.5,126.9,126.4-126.2(\mathrm{~m}), 126.1$, $123.7(\mathrm{q}, J=273.0 \mathrm{~Hz}), 123.0,122.6-122.4(\mathrm{~m}), 121.7(\mathrm{t}, J=246.1 \mathrm{~Hz}), 42.0(\mathrm{t}, J=28.4 \mathrm{~Hz}), 28.0$, 23.1. IR (thin film) $v_{\max } 3062,1693,1622,1488 \mathrm{~cm}^{-1}$. MS (EI) m/z (\%) $338\left(\mathrm{M}^{+}\right), 195,143,130,129$ (100), 115. HRMS (EI) calculated for $\mathrm{C}_{19} \mathrm{H}_{15} \mathrm{~F}_{5}$ : 338.1094; Found: $338.1093\left(\mathrm{M}^{+}\right)$. 


\subsection{EPR experiment}

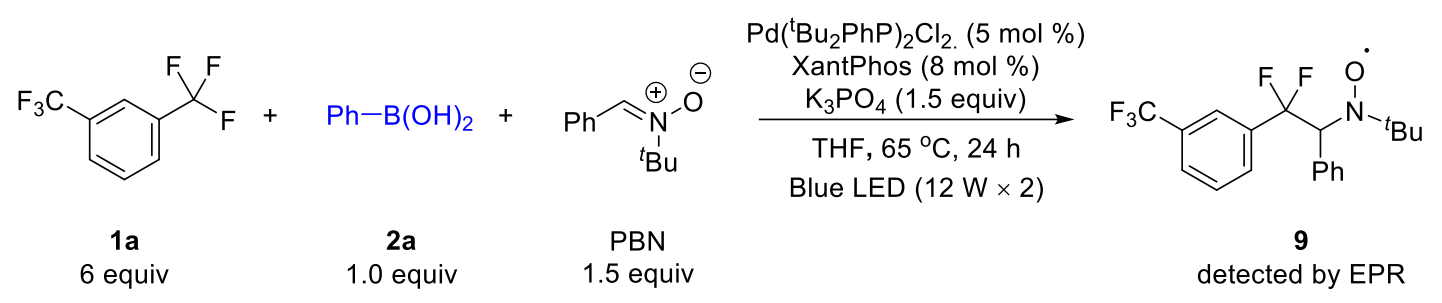

Procedure: To a $25 \mathrm{~mL}$ of Schlenk tube were added $\mathrm{Pd}\left({ }^{t} \mathrm{Bu}_{2} \mathrm{PhP}\right)_{2} \mathrm{Cl}_{2}(9.3 \mathrm{mg}, 0.015 \mathrm{mmol}, 5 \mathrm{~mol} \%)$, Xantphos (13.9 mg, $0.024 \mathrm{mmol}, 8 \mathrm{~mol} \%)$, and $\mathrm{PhB}(\mathrm{OH})_{2}$ 2a $(0.3 \mathrm{mmol}, 1.0$ equiv) in the air. PBN (79.8 $\mathrm{mg}, 0.45 \mathrm{mmol}, 1.5$ equiv) and $\mathrm{K}_{3} \mathrm{PO}_{4}(95.5 \mathrm{mg}, 0.45 \mathrm{mmol}, 1.5$ equiv) were added in the glovebox. The reaction mixture was then evacuated and backfilled with $\operatorname{Ar}(3$ times). 1a (280 $\mu \mathrm{L}, 1.8$ mmol, 6.0 equiv) and THF $(2.0 \mathrm{~mL})$ were added. After pre-stirring at $65{ }^{\circ} \mathrm{C}$ for $20 \mathrm{~min}$, the reaction mixture was stirred and irradiated for $12 \mathrm{~h}$ at $65{ }^{\circ} \mathrm{C}$ under blue LEDs strips $(12 \mathrm{~W} \times 2)$. The reaction mixture was cooled to room temperature and the yield was then analyzed by EPR. Control experiments in the absence of a). $\left.\mathrm{PBN} ; \mathbf{b}) . \mathrm{Ph}-\mathrm{B}(\mathrm{OH})_{2} ; \mathbf{c}\right) \cdot \mathrm{Pd}\left({ }^{t} \mathrm{Bu}_{2} \mathrm{PhP}\right)_{2} \mathrm{Cl}_{2}$ were also conducted. The EPR showed a EPR signal of nitroxide $\left(\mathrm{g}=2.0057, a^{N}=13.9928, a^{H}=2.21432, a^{F}=1.24033\right)$ which indicated that the difluoroalkyl group must be substituted at the $\beta$-carbon atom of nitroxide. And the experimental data of the nitroxide was in accordance with the computer simulation.

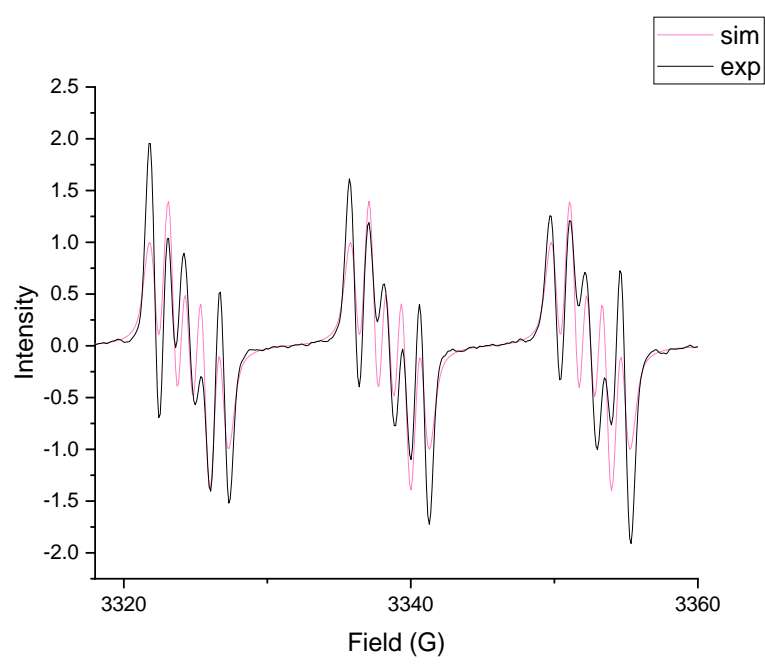

Figure S27. EPR spectra of the reaction mixture: a) experimental data (black line); b) Computer simulation (red line) of the nitroxide. 

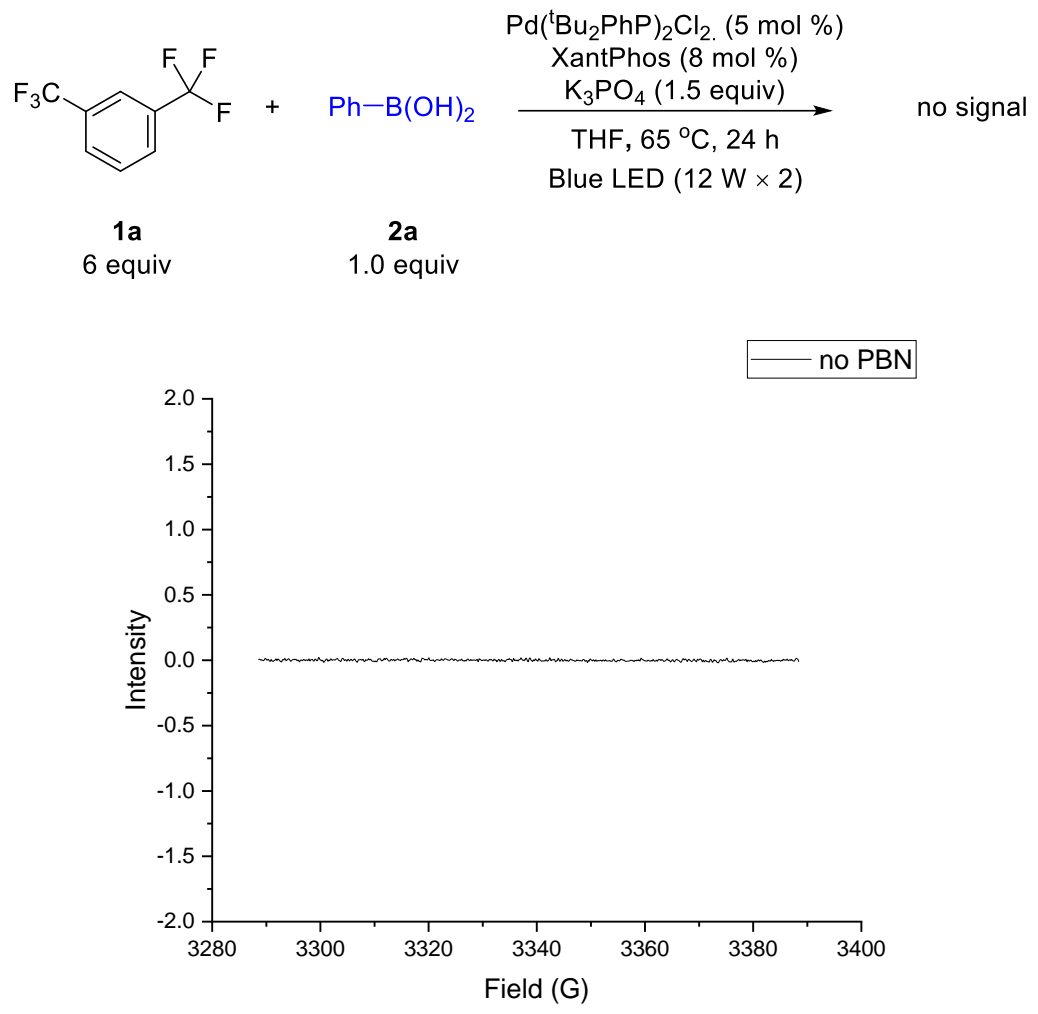

Figure S28. EPR spectrum of reaction mixture in the absence of PBN.
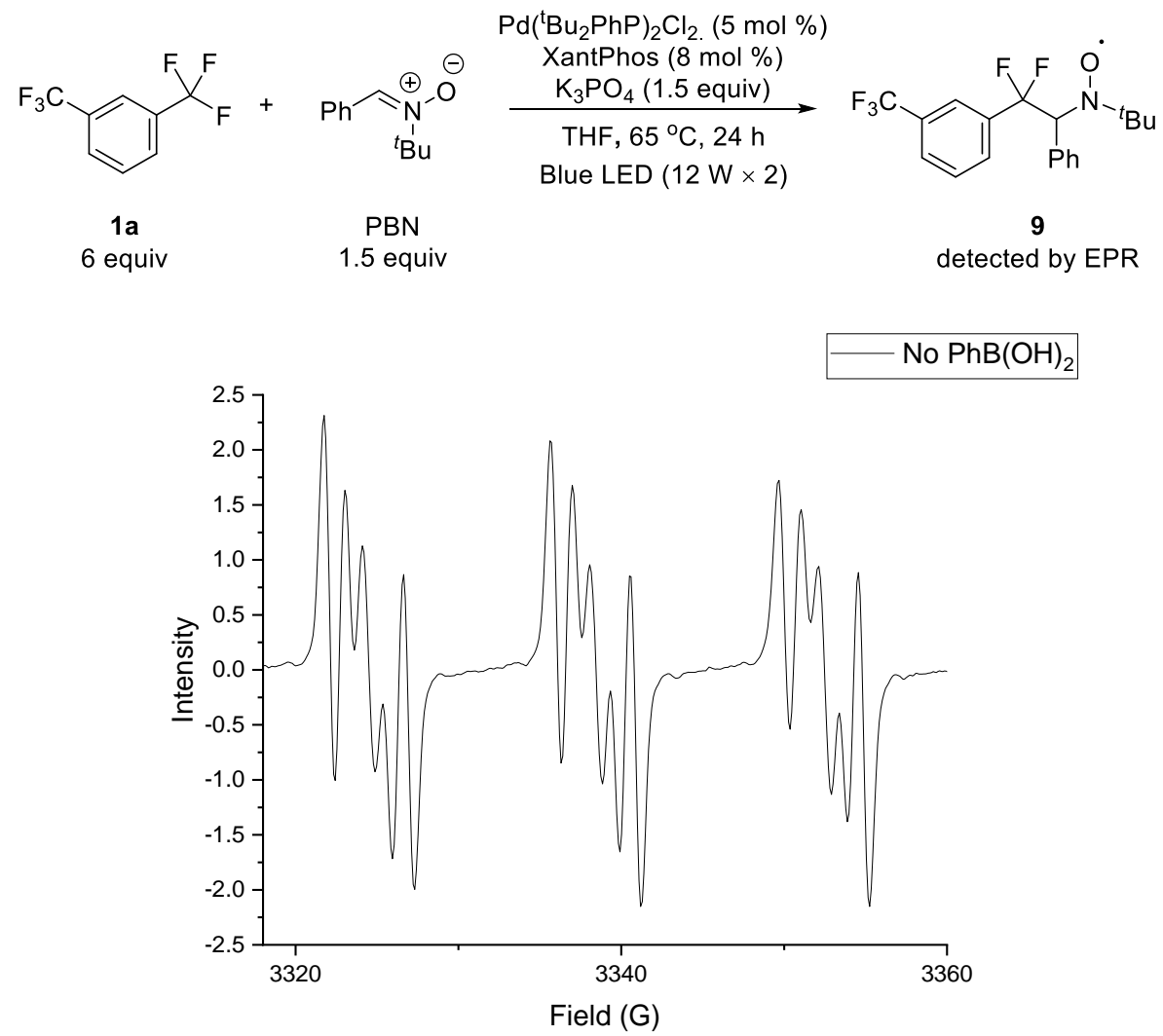

Figure S29. EPR spectrum of reaction mixture in the absence of $\mathrm{Ph}-\mathrm{B}(\mathrm{OH})_{2}$. 


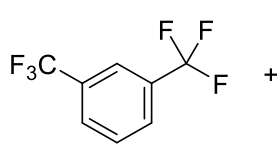

$1 \mathrm{a}$ 6 equiv

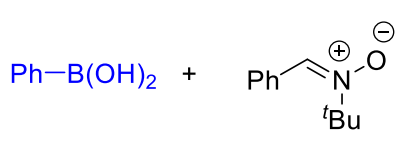

$2 a$

1.0 equiv

PBN

1.5 equiv

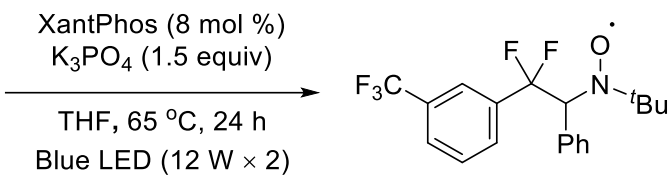

Blue LED $(12 \mathrm{~W} \times 2)$

detected by EPR

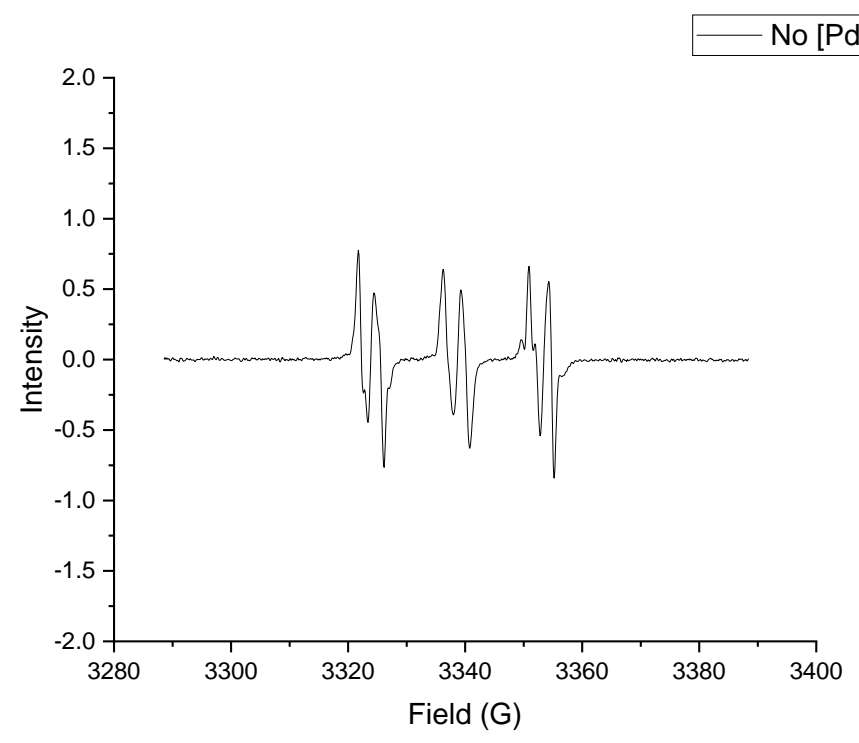

Figure S30. EPR spectrum of reaction mixture in the absence of $\mathrm{Pd}\left({ }^{t} \mathrm{Bu}_{2} \mathrm{PhP}\right){ }_{2} \mathrm{Cl}_{2}$.

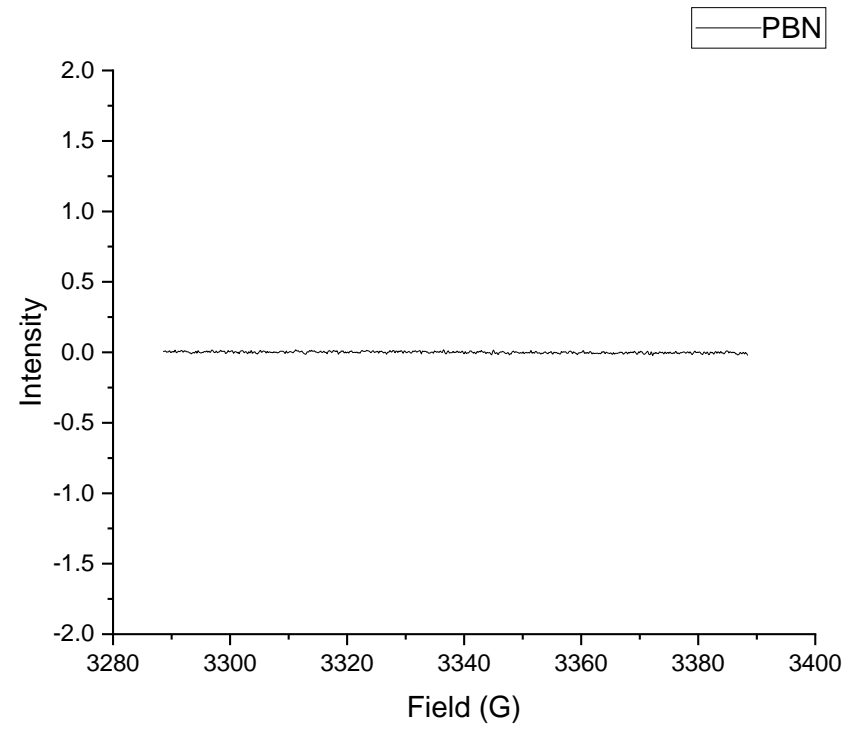

Figure S31. EPR spectrum of PBN. 


\subsection{Dection of fluoride anion}

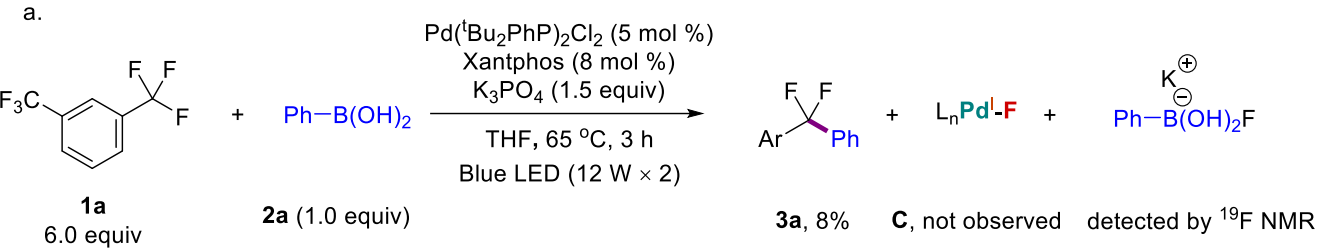

\begin{tabular}{|c|c|c|c|c|}
\hline \multirow{2}{*}{ KF } & \multirow{2}{*}{+} & \multirow{2}{*}{$\mathrm{B}(\mathrm{OH})_{3}$} & $\mathrm{~K}_{3} \mathrm{PO}_{4}$ (1.5 equiv) & \multirow{3}{*}{ no ${ }^{19} \mathrm{~F}$ NMR signal } \\
\hline & & & THF (2 mL), Ar & \\
\hline equiv & & 1.0 equiv & $65^{\circ} \mathrm{C}, 30 \mathrm{~min}$ & \\
\hline
\end{tabular}

c.

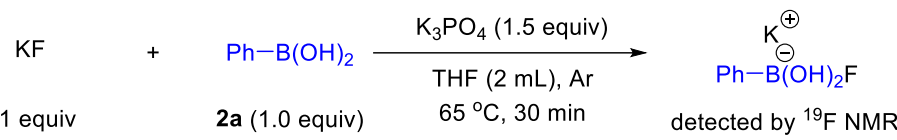

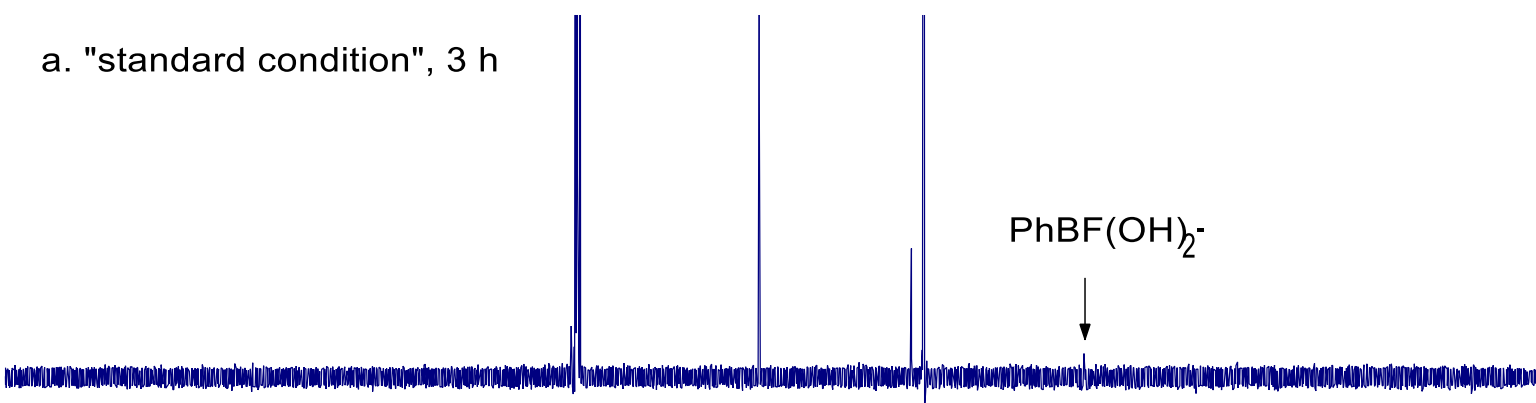

b. $\mathrm{KF}+\mathrm{B}\left(\mathrm{OH}_{3}+\mathrm{K}_{3} \mathrm{PO}_{4}\right.$

c. $\mathrm{KF}+\mathrm{PhB}\left(\mathrm{OH}_{2}+\mathrm{K}_{3} \mathrm{PO}_{4}\right.$

$\operatorname{PhBF}\left(\mathrm{OH}_{2}\right.$

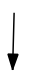

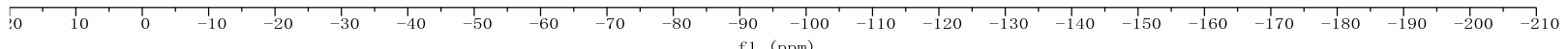




\subsection{The role of arylboronic acid in the $\mathrm{C}-\mathrm{F}$ bond activation}

\section{Catalytic conditions ${ }^{a}$}

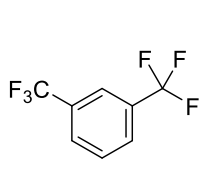

$1 \mathrm{a}$

1.0 equiv
[Pd]. $(5 \mathrm{~mol} \%)$

XantPhos $(8 \mathrm{~mol} \%)$

$\mathrm{K}_{3} \mathrm{PO}_{4}$ (1.5 equiv)

THF, $65^{\circ} \mathrm{C}, 3 \mathrm{~h}$

Blue LED $(12 \mathrm{~W} \times 2)$

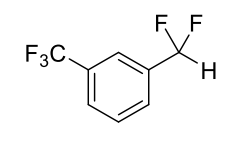

4a, nd

\begin{tabular}{cccc}
\hline entry & {$[\mathrm{Pd}]$} & $\mathbf{1 a}(\%)^{b}$ & $\mathbf{4 a}(\%)^{c}$ \\
\hline 1 & $\mathrm{Pd}\left(t \mathrm{Bu}_{2} \mathrm{PhP}\right)_{2} \mathrm{Cl}_{2}$ & 100 & nd \\
2 & $\mathrm{Pd}\left(\mathrm{PPh}_{3}\right)_{4}$ & $>99$ & $<1$ \\
\hline
\end{tabular}

${ }^{a}$ The yield was determined by ${ }^{19} \mathrm{~F}$ NMR using fluorobenzene as an internal standard. ${ }^{b}$ The yield was calculated based on $\mathbf{1 a} .{ }^{c}$ The yield was calculated based on [Pd].

$\mathrm{PhB}(\mathrm{OH})_{2}$ is not involved in the direct activation of $\mathrm{C}-\mathrm{F}$ bond because $\mathbf{4 a}$ could be generated in the absence of $\mathrm{PhB}(\mathrm{OH})_{2}$. We proposed that one of the roles of $\mathrm{PhB}(\mathrm{OH})_{2}$ is to reduce $\mathrm{Pd}(\mathrm{II})$ to $\mathrm{Pd}(0)$ for C-F bond activation of $\mathrm{ArCF}_{3}$. 


\subsection{Reaction of compounds 4 with arylboronic acids}

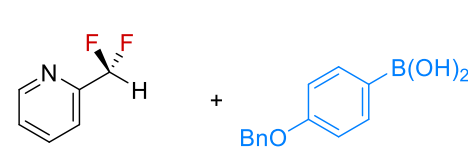

4s 3 equiv

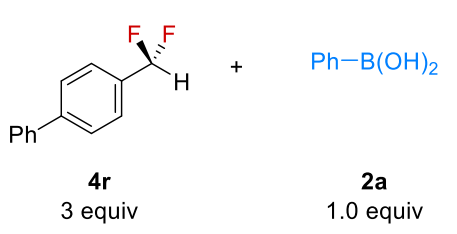

$\mathrm{Pd}\left({ }^{\mathrm{t}} \mathrm{Bu}_{2} \mathrm{PhP}\right)_{2} \mathrm{Cl}_{2}$. (5 mol \%)

XantPhos $(8 \mathrm{~mol} \%)$

$\underset{\mathrm{THF}, 65^{\circ} \mathrm{C}, 24 \mathrm{~h}}{\stackrel{\mathrm{K}_{3} \mathrm{PO}_{4}(1.5 \text { equiv })}{\longrightarrow}}$

Blue LED $(12 \mathrm{~W} \times 2)$

1.0 equiv

$\mathrm{Pd}\left({ }^{\mathrm{t}} \mathrm{Bu}_{2} \mathrm{PhP}\right)_{2} \mathrm{Cl}_{2} .(5 \mathrm{~mol} \%)$

XantPhos $(8 \mathrm{~mol} \%)$

$\mathrm{K}_{3} \mathrm{PO}_{4}$ (1.5 equiv)

THF, $65^{\circ} \mathrm{C}, 24 \mathrm{~h}$

Blue LED $(12 \mathrm{~W} \times 2)$

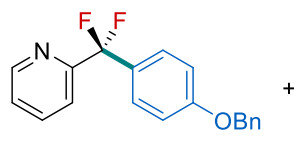

$3 s$, nd

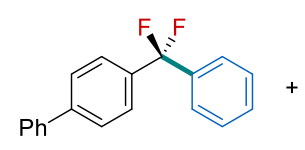

$3 r$, nd

$\mathrm{C}-\mathrm{H}$ cleavage

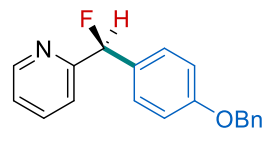

$48 \%$

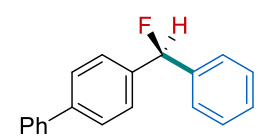

$36 \%$

C-F cleavage

The yield was determined by ${ }^{19} \mathrm{~F}$ NMR using fluorobenzene as an internal standard. The yield was calculated based on 2 .

Compounds 3 were not observed during the reaction process. Instead, the defluoroarylation products were obtained, demonstrating that compounds $\mathbf{3}$ are not the intermediates for the formation of 3 . 


\subsection{Mechanistic studies on the formation of compound 5}

\section{Table S9. Photoinduced C-F bond cleavage of 3a}

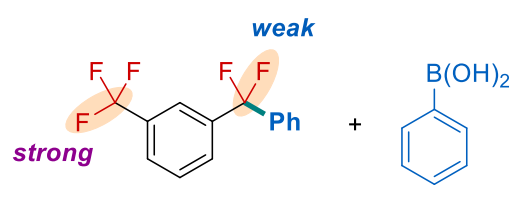

$3 a$ 2a

$\mathrm{Pd}\left({ }^{\mathrm{t}} \mathrm{Bu}_{2} \mathrm{PhP}\right)_{2} \mathrm{Cl}_{2}(5 \mathrm{~mol} \%)$

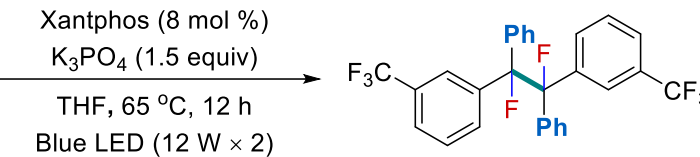

Blue LED $(12 \mathrm{~W} \times 2)$

$\begin{array}{ccc} & \mathbf{5 a} & \mathbf{5 a} \\ \text { blue LED: } & 17 \%^{\mathrm{b}} & \text { nd } \\ \text { no light: } & \text { nd } & \text { nd }\end{array}$

\begin{tabular}{ccccc}
\hline entry & condition & $\mathbf{3 a}^{\mathrm{b}}$ & $\mathbf{5 a}^{\mathrm{b}}$ & $\mathbf{5 a}$ \\
\hline 1 & -- & 66 & 17 & nd \\
2 & No light & 100 & nd & nd \\
\hline
\end{tabular}

${ }^{a}$ Reaction conditions (unless otherwise specified): 3a ( $0.075 \mathrm{mmol}, 1.0$ equiv), 2a (2 equiv). The yield was determined by ${ }^{19} \mathrm{~F}$ NMR using fluorobenzene as an internal standard. ${ }^{b}$ The yield was calculated based on 3a. nd, not detected.

\section{EPR experiment}

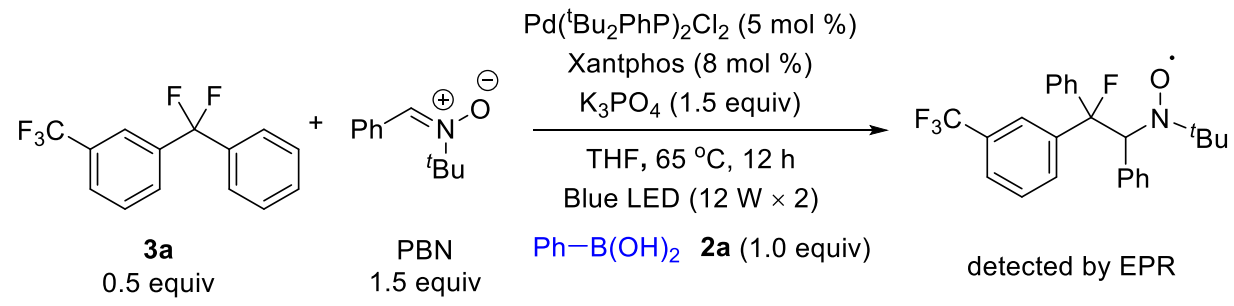

Procedure: To a $25 \mathrm{~mL}$ of Schlenk tube were added $\mathrm{Pd}\left({ }^{t} \mathrm{Bu}_{2} \mathrm{PhP}\right)_{2} \mathrm{Cl}_{2}(4.7 \mathrm{mg}, 0.0075 \mathrm{mmol}, 5 \mathrm{~mol} \%)$, Xantphos (6.9 mg, $0.012 \mathrm{mmol}, 8 \mathrm{~mol} \%)$, and $\mathrm{PhB}(\mathrm{OH})_{2}$ 2a (18.3 mg, $0.15 \mathrm{mmol}, 1.0$ equiv) in the air. PBN (39.9 mg, 0.225 mmol, 1.5 equiv) and $\mathrm{K}_{3} \mathrm{PO}_{4}$ (47.8 mg, $0.225 \mathrm{mmol}, 1.5$ equiv) were added in the glovebox. The reaction mixture was then evacuated and backfilled with Ar (3 times). 3a (19.8 $\mathrm{mg}, 0.075 \mathrm{mmol}, 0.5$ equiv) and THF $(1.0 \mathrm{~mL})$ were added. After pre-stirring at $65^{\circ} \mathrm{C}$ for $20 \mathrm{~min}$, the reaction mixture was stirred and irradiated for $12 \mathrm{~h}$ at $65{ }^{\circ} \mathrm{C}$ under blue LEDs strips $(12 \mathrm{~W} \times 2)$. The reaction mixture was cooled to room temperature and the yield was then analyzed by EPR. The EPR showed a EPR signal of nitroxide $\left(\mathrm{g}=2.00534, a^{N}=14.6509, a^{H}=2.34\right)$, and the experimental data of the nitroxide was in accordance with the computer simulation. 


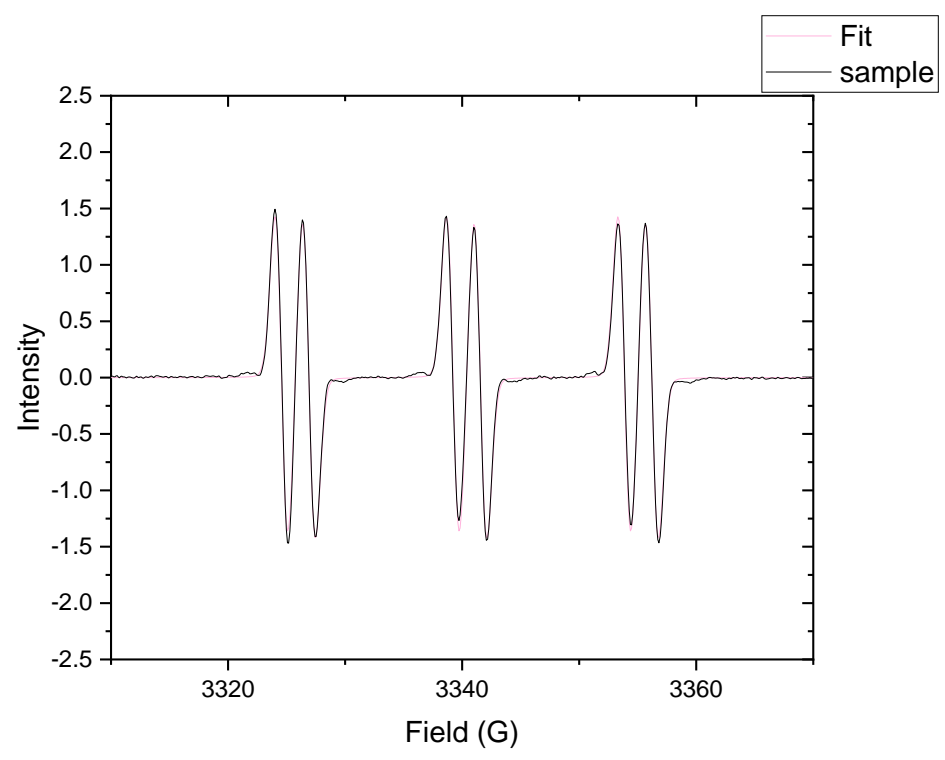

Figure S32. EPR spectra of the reaction mixture: a) experimental data (black line); b) Computer simulation (red line) of the nitroxide.

Table S10. Real-time monitor of the standard reaction
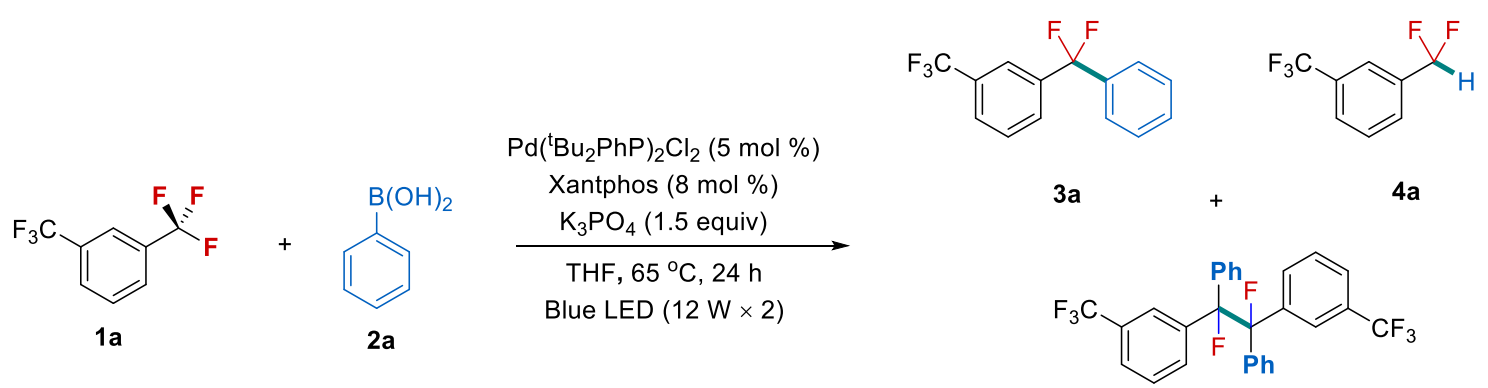

$5 a$

\begin{tabular}{ccccc}
\hline entry & Conditions & $\mathbf{3 a}(\%)^{b}$ & $\mathbf{4 a}(\%)^{c}$ & $\mathbf{5 a}(\%)^{b}$ \\
\hline 1 & $0.5 \mathrm{~h}$ & 14 & $<1$ & 0 \\
2 & $2 \mathrm{~h}$ & 32 & $<1$ & 0 \\
3 & $6 \mathrm{~h}$ & 59 & 1 & 3 \\
4 & $12 \mathrm{~h}$ & 60 & 1 & 5 \\
5 & $24 \mathrm{~h}$ & 60 & 1 & 5 \\
\hline
\end{tabular}

${ }^{a}$ Reaction conditions (unless otherwise specified): 1a (6.0 equiv), 2a (0.3 mmol, 1.0 equiv). The yield was determined by ${ }^{19} \mathrm{~F}$ NMR using fluorobenzene as an internal standard. ${ }^{b}$ The yield was calculated based on $\mathbf{2 a} .{ }^{c}$ The yield was calculated based on $\mathbf{1 a}$. nd, not detected. 


\subsection{The selectivities of $\mathrm{C}-\mathrm{F}$ bond activation between 1a and 3a}

\section{Kinetic studies}

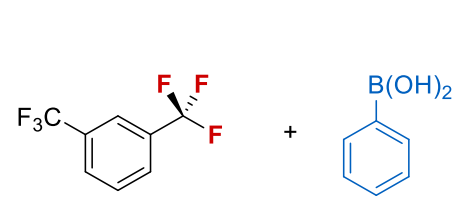

$1 \mathrm{a}$

0.5 equiv

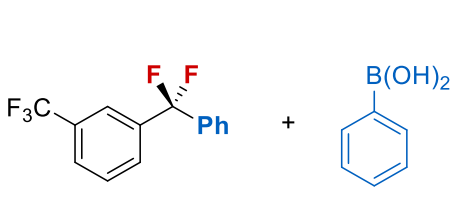

$3 a$

0.5 equiv
$\mathrm{Pd}\left({ }^{\mathrm{t}} \mathrm{Bu}_{2} \mathrm{PhP}\right)_{2} \mathrm{Cl}_{2}(5 \mathrm{~mol} \%)$

Xantphos (8 mol \%)

$\frac{\mathrm{K}_{3} \mathrm{PO}_{4}(1.5 \text { equiv })}{\mathrm{THF}, 65^{\circ} \mathrm{C}, \text { time }}$

Blue LED $(12 \mathrm{~W} \times 2)$

$\mathrm{Pd}\left({ }^{\mathrm{t}} \mathrm{Bu}_{2} \mathrm{PhP}\right)_{2} \mathrm{Cl}_{2}(5 \mathrm{~mol} \%)$

Xantphos (8 $\mathrm{mol} \%$ )

$\mathrm{K}_{3} \mathrm{PO}_{4}$ (1.5 equiv)

THF, $65^{\circ} \mathrm{C}$, time

Blue LED $(12 \mathrm{~W} \times 2)$

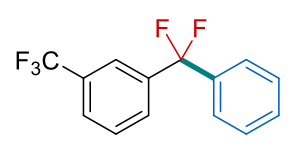

$3 a$

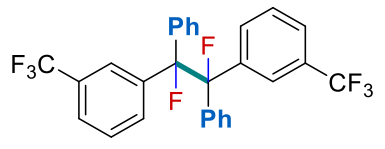

$5 a$

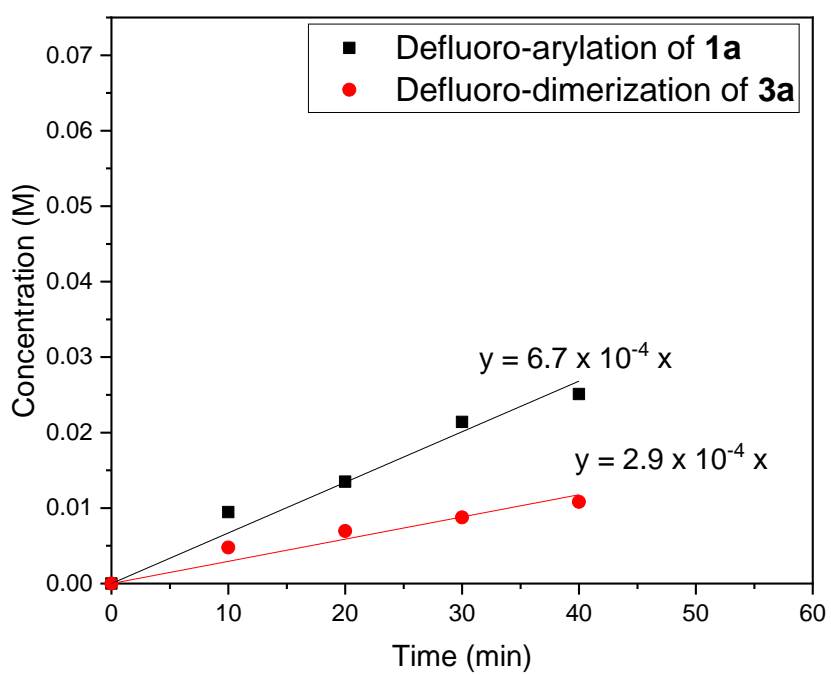

Figure S33. Kinetics of defluoro-arylation of 1a (black line, 1a (0.5 equiv), 2a (1.0 equiv)) and defluoro-dimerization of $\mathbf{3 a}$ (red line: $\mathbf{3 a}$ ( 0.5 equiv), $\mathbf{2 a}$ (1.0 equiv)). The decreased concertration of starting materials $\mathbf{1 a} / \mathbf{3 a}$ was ploted vs time.

Procedure: To a $25 \mathrm{~mL}$ of Schlenk tube were added $\mathrm{Pd}\left({ }^{t} \mathrm{Bu}_{2} \mathrm{PhP}\right)_{2} \mathrm{Cl}_{2}(9.3 \mathrm{mg}, 0.015 \mathrm{mmol}, 5 \mathrm{~mol} \%)$, Xantphos (13.9 mg, $0.024 \mathrm{mmol}, 8 \mathrm{~mol} \%)$, and $\mathrm{PhB}(\mathrm{OH})_{2} \mathbf{2 a}\left(0.3 \mathrm{mmol}, 1.0\right.$ equiv) in the air. $\mathrm{K}_{3} \mathrm{PO}_{4}$ (95.5 $\mathrm{mg}, 0.45 \mathrm{mmol}, 1.5$ equiv) was then added in the glovebox. The reaction mixture was evacuated and backfilled with $\operatorname{Ar}$ (3 times). 1a or $3 \mathbf{a}(0.15 \mathrm{mmol}, 0.5$ equiv) and THF ( $2.0 \mathrm{~mL})$ were added. After pre-stirring at $65^{\circ} \mathrm{C}$ for $20 \mathrm{~min}$, the reaction mixture was stirred and irradiated for 10/20/30/40 mins at $65{ }^{\circ} \mathrm{C}$ under blue LED strips $(12 \mathrm{~W} \times 2)$. The reaction mixture was then cooled to room temperature and the yield was determined by ${ }^{19} \mathrm{~F}$ NMR using fluorobenzene as an internal standard. 


\section{DFT calculation of C-F bond BDE}

All DFT calculations were carried out using the Gaussian 09w software package at the UB3LYP level of theory with the 6-311+G(d,p) basis. Bond dissociation energies were calculated using the following equation:

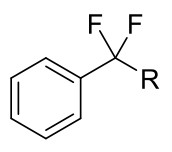

$\mathrm{ArCF}_{2} \mathrm{R}$

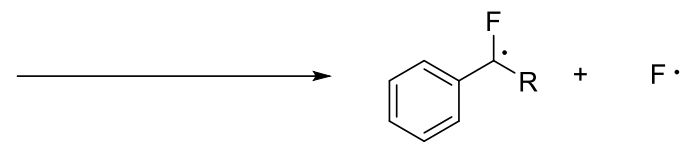

ArCFR・<smiles>FC(F)(F)c1ccccc1</smiles>

BDE $(\mathrm{kcal} / \mathrm{mol})$<smiles>CC(F)(F)c1ccccc1</smiles>

102<smiles>FC(F)c1ccccc1</smiles>

101<smiles>FC(F)(c1ccccc1)c1ccccc1</smiles>

95

$\mathrm{BDE}=\mathrm{E}(\mathrm{ArCFR} \bullet)+\mathrm{E}(\mathrm{F} \bullet)-\mathrm{E}\left(\mathrm{ArCF}_{2} \mathrm{R}\right)$

\section{$\mathrm{PhCF}_{3}$}

$\begin{array}{lrrr}\text { C } & 0.75539800 & 1.21995103 & -0.01670154 \\ \text { C } & 2.15193600 & 1.20687752 & 0.00153242 \\ \text { C } & 2.84234168 & -0.00735491 & 0.01100247 \\ \text { C } & 2.13564695 & -1.21399117 & 0.00135138 \\ \text { C } & 0.74088813 & -1.20819124 & -0.01721034 \\ \text { C } & 0.05304264 & 0.01117478 & -0.02743098 \\ \text { H } & 0.21558819 & 2.16029047 & -0.02799343 \\ \text { H } & 2.69790471 & 2.14530398 & 0.00639437 \\ \text { H } & 3.92824385 & -0.01459372 & 0.02400376 \\ \text { H } & 2.66993568 & -2.15911274 & 0.00602013 \\ \text { H } & 0.18931271 & -2.14261808 & -0.02873418 \\ \text { C } & -1.45238190 & 0.00547480 & -0.00346678 \\ \text { F } & -1.93825949 & -0.24751882 & 1.24378899 \\ \text { F } & -1.98373489 & 1.19022859 & -0.39262815\end{array}$


$\begin{array}{lll}-1.97380719 & -0.95081141 & -0.81495533\end{array}$

$\mathrm{PhCF}_{2} \bullet$

C

C

C

C

$\mathrm{C}$

C

$\mathrm{H}$

$\mathrm{H}$

$\mathrm{H}$

$\mathrm{H}$

$\mathrm{H}$

C

F

F

\begin{tabular}{rrr}
-0.51285323 & 1.22435988 & 0.03117229 \\
-1.90105701 & 1.21202435 & -0.01524254 \\
-2.60583837 & -0.00002084 & -0.03715352 \\
-1.90112100 & -1.21198727 & -0.01521473 \\
-0.51278789 & -1.22440914 & 0.03132982 \\
0.20732816 & -0.00014956 & 0.05175139 \\
0.02866899 & 2.16390656 & 0.04797847 \\
-2.44183949 & 2.15365418 & -0.03699378 \\
-3.69080961 & -0.00000971 & -0.07119583 \\
-2.44186320 & -2.15363459 & -0.03683302 \\
0.02856353 & -2.16408274 & 0.04818943 \\
1.61812978 & 0.00003263 & 0.16219776 \\
\hline 2.34272983 & -1.10165033 & -0.06701354 \\
2.34243430 & 1.10176878 & -0.06678513
\end{tabular}

$\mathrm{PhCF}_{2} \mathrm{Me}$

C

C

C

C

C

C

$\mathrm{H}$

$\mathrm{H}$

$\mathrm{H}$

$\mathrm{H}$ $\begin{array}{lll}0.76721362 & -1.21063550 & -0.01406258\end{array}$

$\begin{array}{lll}2.16318570 & -1.20883936 & 0.02952360\end{array}$

$\begin{array}{lll}2.86404750 & -0.00011707 & 0.05284028\end{array}$

$\begin{array}{lll}2.16333488 & 1.20870309 & 0.02983588\end{array}$

$\begin{array}{lll}0.76736524 & 1.21070202 & -0.01375575\end{array}$

$\begin{array}{lll}0.06455760 & 0.00008244 & -0.03753081\end{array}$

$\begin{array}{lll}0.22413076 & -2.14943544 & -0.04535244\end{array}$

$\begin{array}{lll}2.70194951 & -2.15176922 & 0.03993043\end{array}$

$\begin{array}{lll}3.94967113 & -0.00018200 & 0.08450882\end{array}$

$\begin{array}{lll}2.70223492 & 2.15155226 & 0.04047049\end{array}$ 


$\begin{array}{lrrr}\mathrm{H} & 0.22438784 & 2.14956184 & -0.04476447 \\ \mathrm{C} & -1.45089061 & 0.00009400 & -0.03122819 \\ \mathrm{C} & -2.10401940 & -0.00181472 & 1.33739524 \\ \mathrm{H} & -1.79819037 & 0.88619049 & 1.89583335 \\ \mathrm{H} & -3.19062471 & -0.00177548 & 1.21828274 \\ \mathrm{H} & -1.79798114 & -0.89116548 & 1.89356032 \\ \mathrm{~F} & -1.91242481 & -1.10494866 & -0.73489870 \\ \mathrm{~F} & -1.91250242 & 1.10694573 & -0.73183188\end{array}$

\section{PhCFMe•}

C

C

C

C

C

C

$\mathrm{H}$

$\mathrm{H}$

$\mathrm{H}$

$\mathrm{H}$

$\mathrm{H}$

C

C

$\mathrm{H}$

$\mathrm{H}$

$\mathrm{H}$

F
$0.52743552 \quad 1.24044661 \quad-0.00006912$

$\begin{array}{lll}1.91507460 & 1.25832374 & -0.00006107\end{array}$

$\begin{array}{lll}2.64973301 & 0.06292619 & 0.00000335\end{array}$

$\begin{array}{lll}1.96476463 & -1.16168572 & 0.00007634\end{array}$

$\begin{array}{lll}0.57781852 & -1.20194776 & 0.00007341\end{array}$

$\begin{array}{lll}-0.18769120 & 0.00415499 & -0.00001180\end{array}$

$\begin{array}{lll}-0.01422568 & 2.18033611 & -0.00011987\end{array}$

$\begin{array}{lll}2.43440916 & 2.21263173 & -0.00011371\end{array}$

$\begin{array}{lll}3.73498190 & 0.08591211 & 0.00000578\end{array}$

$\begin{array}{lll}2.52359051 & -2.09346241 & 0.00012992\end{array}$

$\begin{array}{lll}0.05922418 & -2.15356073 & 0.00013022\end{array}$

$\begin{array}{lll}-1.59663760 & -0.03649996 & -0.00000134\end{array}$

$\begin{array}{lll}-2.60739189 & 1.04891733 & 0.00012961\end{array}$

$\begin{array}{lll}-2.13989746 & 2.03437186 & 0.00026514\end{array}$

$\begin{array}{lll}-3.25613498 & 0.97669869 & -0.88325683\end{array}$

$\begin{array}{lll}-3.25615967 & 0.97646723 & 0.88347868\end{array}$

$\begin{array}{lll}-2.17160240 & -1.27857857 & -0.00015062\end{array}$ 


\section{$\mathrm{PhCF}_{2} \mathrm{H}$}

\begin{tabular}{lrrr} 
C & 0.52145376 & 1.21162594 & 0.08754562 \\
C & 1.91208094 & 1.21008948 & -0.04164960 \\
C & 2.61071842 & 0.00134793 & -0.10244066 \\
C & 1.91444180 & -1.20855765 & -0.03603112 \\
C & 0.52390961 & -1.21215590 & 0.09310851 \\
H & -0.17613036 & -0.00076565 & 0.15063445 \\
H & -0.02228655 & 2.14972908 & 0.12575131 \\
H & 2.44794919 & 2.15296986 & -0.09830335 \\
H & 3.69210386 & 0.00216687 & -0.20363175 \\
H & 2.45218919 & -2.15062055 & -0.08830030 \\
C & -0.01790709 & -2.15125907 & 0.13542661 \\
H & -1.67063697 & -0.00270071 & 0.34811780 \\
F & -1.98479108 & -0.01008149 & 1.39768321 \\
F & -2.24274707 & -1.10863399 & -0.24439772 \\
\hline & -2.24417301 & 1.11016673 & -0.22941736
\end{tabular}

\section{PhCFH•}

C

C

C

C

C

$\mathrm{H}$

$\mathrm{H}$

$\mathrm{H}$

$\mathrm{H}$

$\mathrm{H}$

C $\begin{array}{lll}-0.45989995 & 1.31478667 & 0.00000010\end{array}$

$\begin{array}{lll}-1.81575174 & 1.02386467 & -0.00001265\end{array}$

$\begin{array}{lll}-2.26002967 & -0.30857085 & -0.00000961\end{array}$

$\begin{array}{lll}-1.32075296 & -1.35124338 & 0.00000539\end{array}$

$\begin{array}{lll}0.04124654 & -1.08206240 & 0.00001697\end{array}$

$\begin{array}{lll}-0.12365331 & 2.34826391 & 0.00000059\end{array}$

$\begin{array}{lll}-2.53793808 & 1.83529976 & -0.00002183\end{array}$

$\begin{array}{lll}-3.32277440 & -0.52977682 & -0.00001705\end{array}$

$\begin{array}{lll}-1.66050137 & -2.38313794 & 0.00000855\end{array}$

$\begin{array}{lll}0.76346637 & -1.89101711 & 0.00003050\end{array}$

$\begin{array}{lll}0.50700932 & 0.26534401 & 0.00001184\end{array}$ 


$\begin{array}{lrrr}\mathrm{C} & 1.87247302 & 0.58423772 & 0.00002799 \\ \mathrm{~F} & 2.79896368 & -0.40382065 & -0.00003274 \\ \mathrm{H} & 2.30496032 & 1.57661537 & 0.00005376\end{array}$

$\mathrm{PhCF}_{2} \mathrm{Ph}$

\begin{tabular}{|c|c|c|c|}
\hline $\mathrm{C}$ & -2.53704297 & 0.79585271 & 0.16915837 \\
\hline $\mathrm{C}$ & -3.70675001 & 0.03823715 & 0.16084642 \\
\hline $\mathrm{C}$ & -3.64575898 & -1.34699735 & -0.02926297 \\
\hline $\mathrm{C}$ & -2.40964133 & -1.96831075 & -0.21085102 \\
\hline $\mathrm{C}$ & -1.23401620 & -1.21060458 & -0.20180234 \\
\hline $\mathrm{C}$ & -1.29249983 & 0.17333559 & -0.01159120 \\
\hline $\mathrm{H}$ & -2.58252470 & 1.86955602 & 0.31812385 \\
\hline $\mathrm{H}$ & -4.66605214 & 0.52696626 & 0.30351532 \\
\hline $\mathrm{H}$ & -4.55816839 & -1.93628246 & -0.03431676 \\
\hline $\mathrm{H}$ & -2.35465270 & -3.04289163 & -0.35777148 \\
\hline $\mathrm{H}$ & -0.27627264 & -1.70029493 & -0.34052112 \\
\hline $\mathrm{C}$ & -0.04619236 & 1.03973325 & -0.02858883 \\
\hline $\mathrm{C}$ & 1.29441707 & 0.33685443 & 0.00498473 \\
\hline $\mathrm{C}$ & 2.00909324 & 0.10914299 & -1.17617352 \\
\hline $\mathrm{C}$ & 1.81153481 & -0.10884508 & 1.22812768 \\
\hline $\mathrm{C}$ & 3.23537905 & -0.55983436 & -1.13268568 \\
\hline $\mathrm{H}$ & 1.61240816 & 0.46268218 & -2.12145847 \\
\hline $\mathrm{C}$ & 3.03719645 & -0.77566632 & 1.26767452 \\
\hline $\mathrm{H}$ & 1.26070848 & 0.07499805 & 2.14482717 \\
\hline $\mathrm{C}$ & 3.75090685 & -1.00390822 & 0.08722758 \\
\hline $\mathrm{H}$ & 3.78798710 & -0.72862742 & -2.05222685 \\
\hline $\mathrm{H}$ & 3.43565741 & -1.11304172 & 2.21991919 \\
\hline $\mathrm{H}$ & 4.70509784 & -1.52174830 & 0.11941841 \\
\hline
\end{tabular}




$\begin{array}{llrr}\text { F } & -0.11274288 & 1.92098053 & 1.04761062 \\ \text { F } & -0.10547301 & 1.85621361 & -1.15448748\end{array}$

\section{$\mathrm{Ph}_{2} \mathrm{CF} \bullet$}

\begin{tabular}{|c|c|c|c|}
\hline $\mathrm{C}$ & -1.54983924 & -1.08036082 & 0.43491553 \\
\hline $\mathrm{C}$ & -2.83942913 & -1.60130182 & 0.44960408 \\
\hline $\mathrm{C}$ & -3.93233100 & -0.81601788 & 0.06271325 \\
\hline $\mathrm{C}$ & -3.71804510 & 0.51218369 & -0.32672456 \\
\hline $\mathrm{C}$ & -2.43482408 & 1.04636671 & -0.34603194 \\
\hline $\mathrm{C}$ & -1.31187912 & 0.25612702 & 0.01837055 \\
\hline $\mathrm{H}$ & -0.72797022 & -1.69075723 & 0.78913717 \\
\hline $\mathrm{H}$ & -2.99625991 & -2.62345255 & 0.78232075 \\
\hline $\mathrm{H}$ & -4.93634905 & -1.22897534 & 0.07655130 \\
\hline $\mathrm{H}$ & -4.55896477 & 1.13455991 & -0.61917018 \\
\hline $\mathrm{H}$ & -2.27968862 & 2.07473905 & -0.65096729 \\
\hline $\mathrm{C}$ & 0.00004420 & 0.83581539 & 0.00048400 \\
\hline $\mathrm{C}$ & 1.31185611 & 0.25607946 & -0.01802825 \\
\hline $\mathrm{C}$ & 1.54957736 & -1.08066544 & -0.43407997 \\
\hline $\mathrm{C}$ & 2.43511285 & 1.04644165 & 0.34531521 \\
\hline $\mathrm{C}$ & 2.83913776 & -1.60160359 & -0.44931021 \\
\hline $\mathrm{H}$ & 0.72749419 & -1.69124833 & -0.78748172 \\
\hline $\mathrm{C}$ & 3.71829066 & 0.51222387 & 0.32545505 \\
\hline $\mathrm{H}$ & 2.28019440 & 2.07495044 & 0.64987531 \\
\hline $\mathrm{C}$ & 3.93231928 & -0.81616838 & -0.06347629 \\
\hline $\mathrm{H}$ & 2.99574499 & -2.62392416 & -0.78161369 \\
\hline $\mathrm{H}$ & 4.55940929 & 1.13472511 & 0.61706847 \\
\hline $\mathrm{H}$ & 4.93631655 & -1.22915814 & -0.07771774 \\
\hline $\mathrm{F}$ & 0.00001442 & 2.20598021 & 0.00075099 \\
\hline
\end{tabular}




\section{References}

(1) Luo, C.; Bandar, J. S. Selective Defluoroallylation of Trifluoromethylarenes. J. Am. Chem. Soc. 2019, $141,14120-14125$.

(2) Cheng, G.; Wang, P.; Yu, J.-Q. meta-C-H Arylation and Alkylation of Benzylsulfonamide Enabled by a Palladium(II)/Isoquinoline Catalyst. Angew. Chem., Int. Ed. 2017, 56, 8183-8186.

(3) Wang, H.; Jui, N. T. Catalytic Defluoroalkylation of Trifluoromethylaromatics with Unactivated Alkenes. J. Am. Chem. Soc. 2018, 140, 163-166.

(4) Schneider, C.; Broda, E.; Snieckus, V. Directed ortho-Metalation-Cross-Coupling Strategies. OnePot Suzuki Reaction to Biaryl and Heterobiaryl Sulfonamides. Org. Lett. 2011, 13, 3588-3591. 
8. Copies of ${ }^{1} \mathrm{H}$ NMR, ${ }^{19} \mathrm{~F}$ NMR and ${ }^{13} \mathrm{C}$ NMR Spectra

1-(Methoxymethyl)-3,5-bis(trifluoromethyl)benzene (1m)

${ }^{1} \mathrm{H}$ NMR of $\mathbf{1 m}$
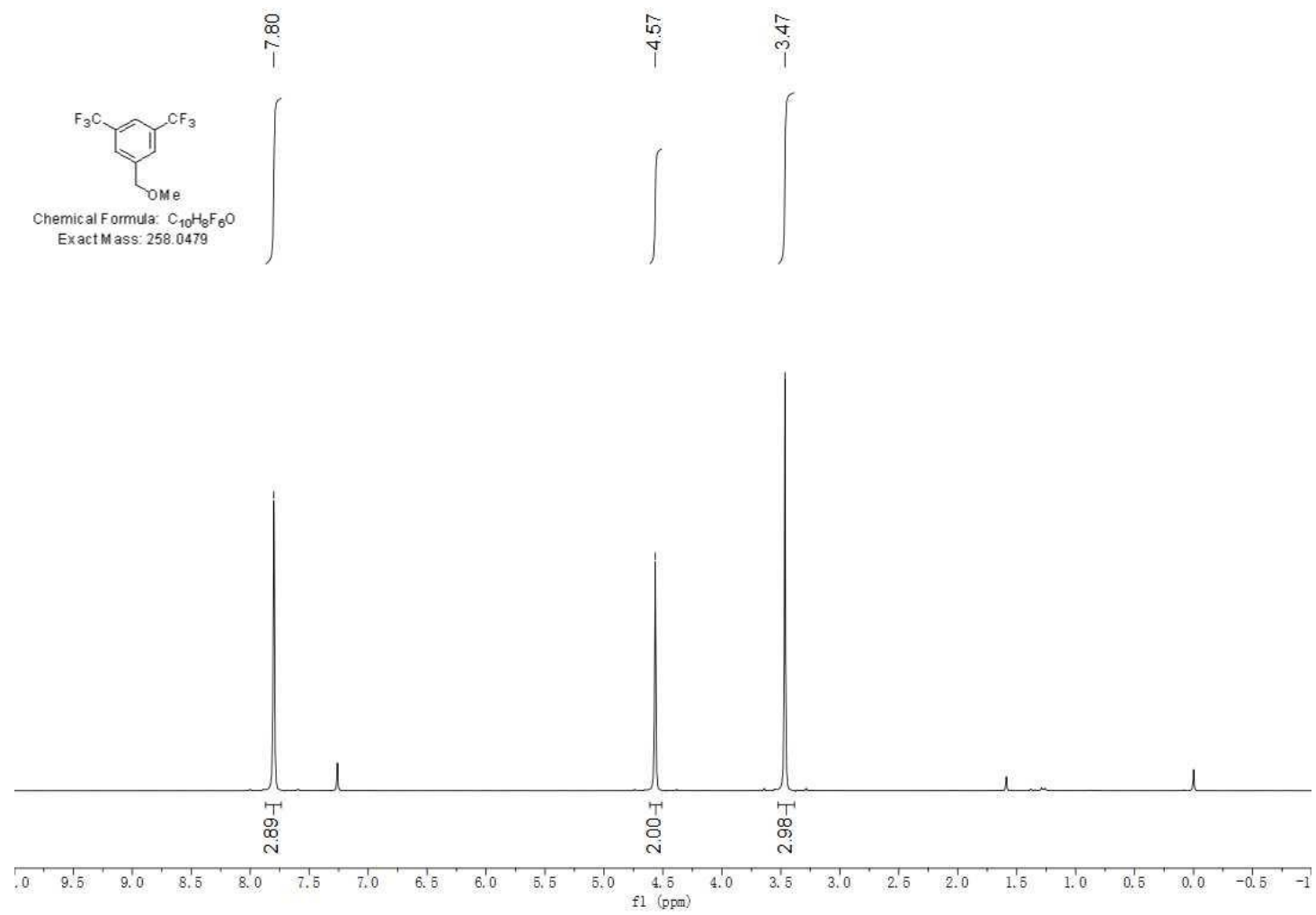

${ }^{19} \mathrm{~F}$ NMR of $\mathbf{1 m}$

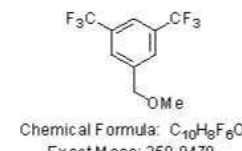

Exactmass: 258.0479

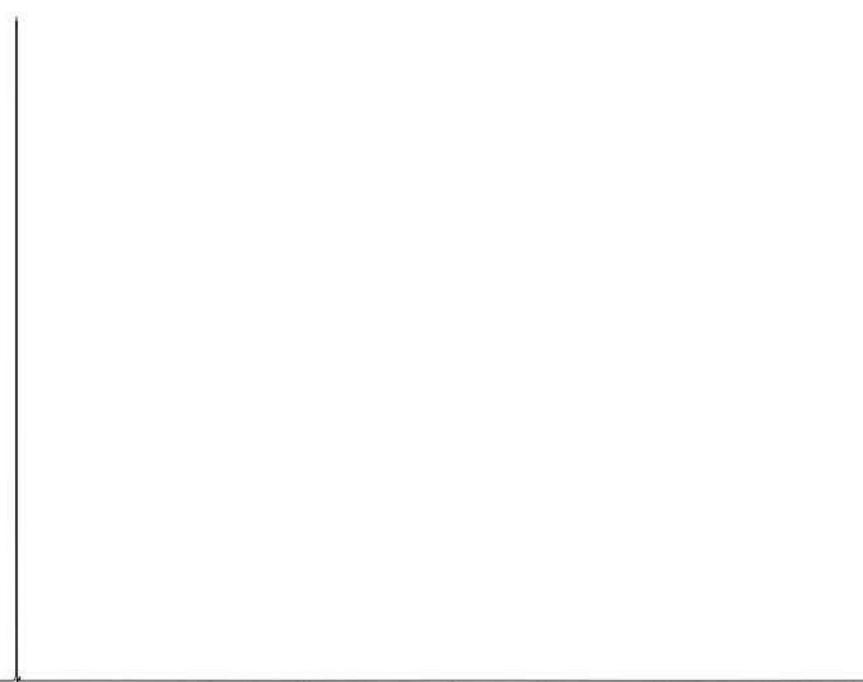

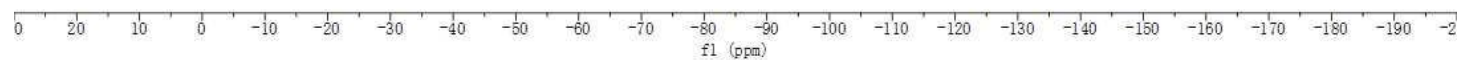


${ }^{13} \mathrm{C}$ NMR of $\mathbf{1 m}$

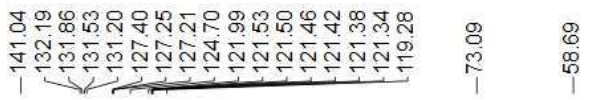

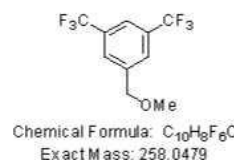

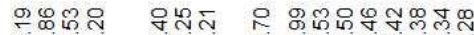

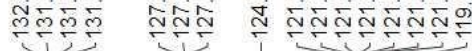

Exact Mass: 258.0479
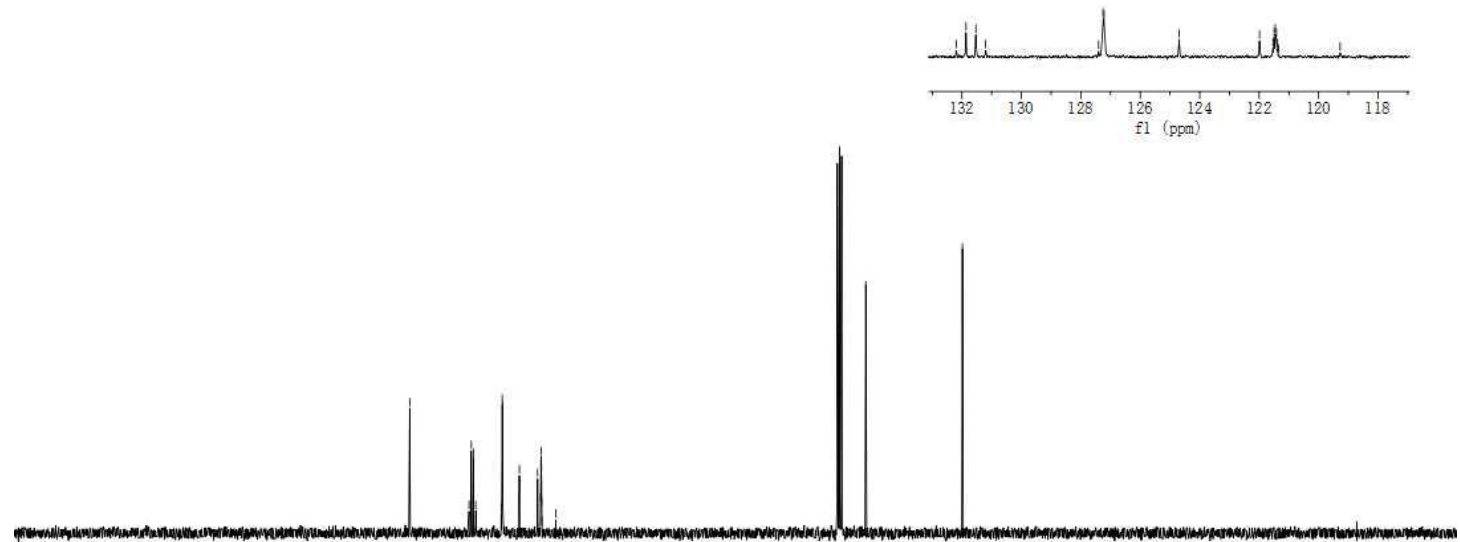

1-((3-chloropropoxy)methyl)-3,5-bis(trifluoromethyl)benzene (1n)

${ }^{1} \mathrm{H}$ spectra of $\mathbf{1 n}$

i
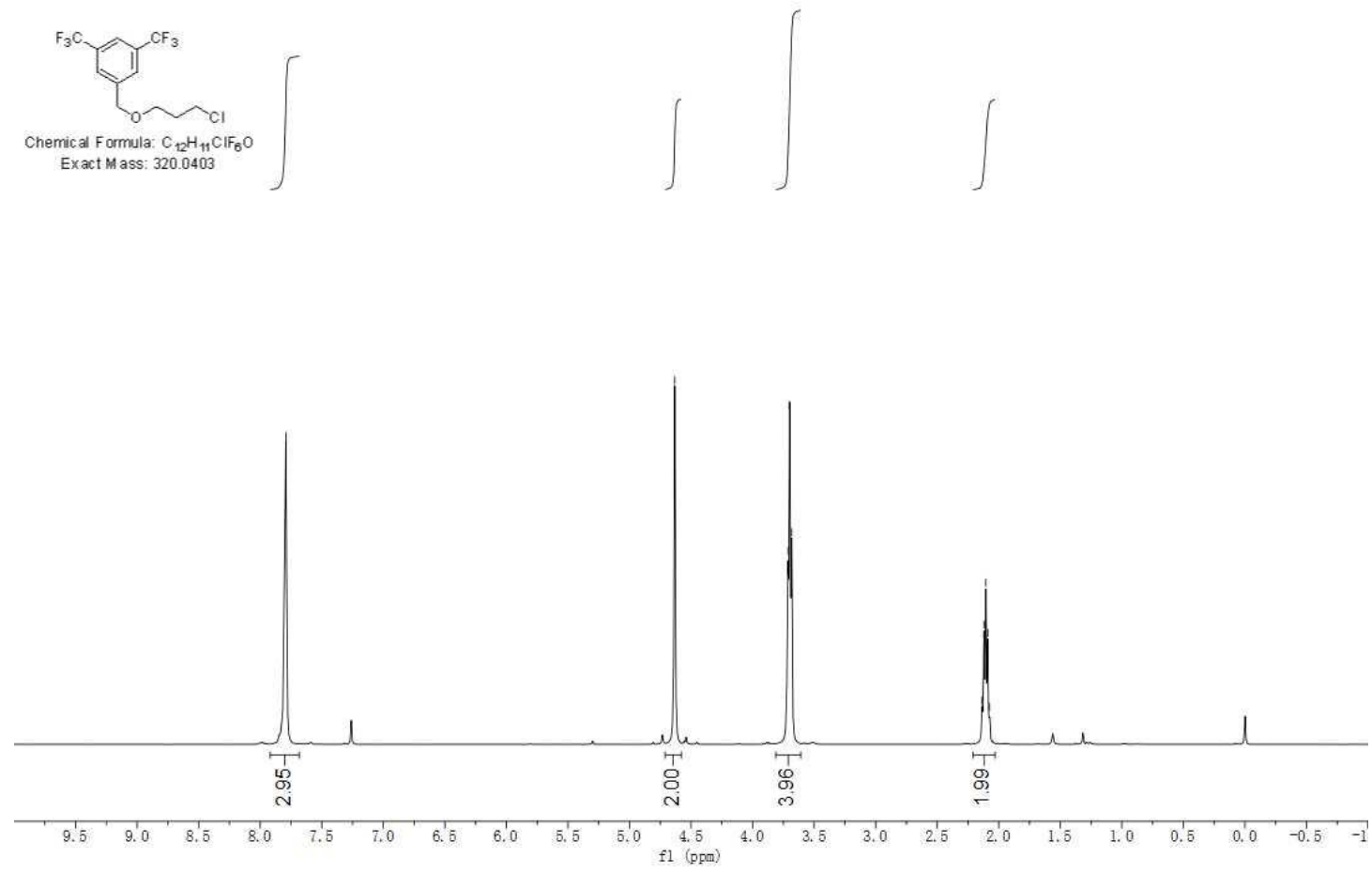
${ }^{19} \mathrm{~F}$ spectra of $\mathbf{1 n}$

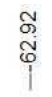

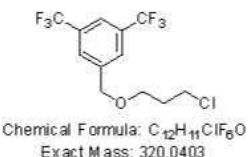

Exact Mass: 320.0403

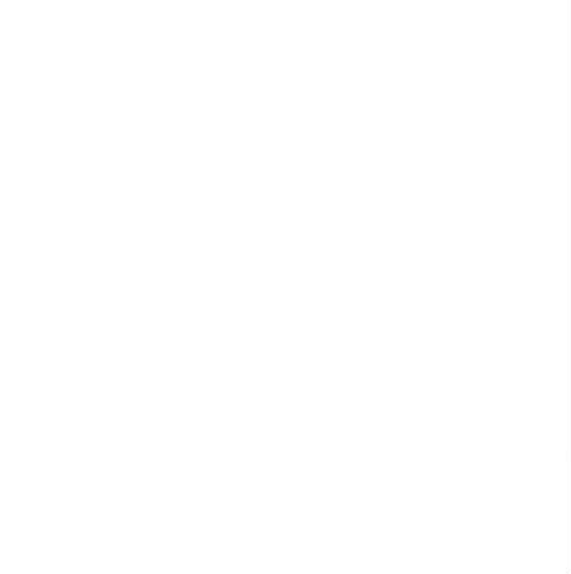

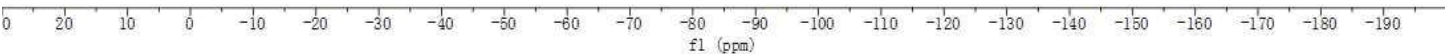

\section{${ }^{13} \mathrm{C}$ spectra of $\mathbf{1 n}$}

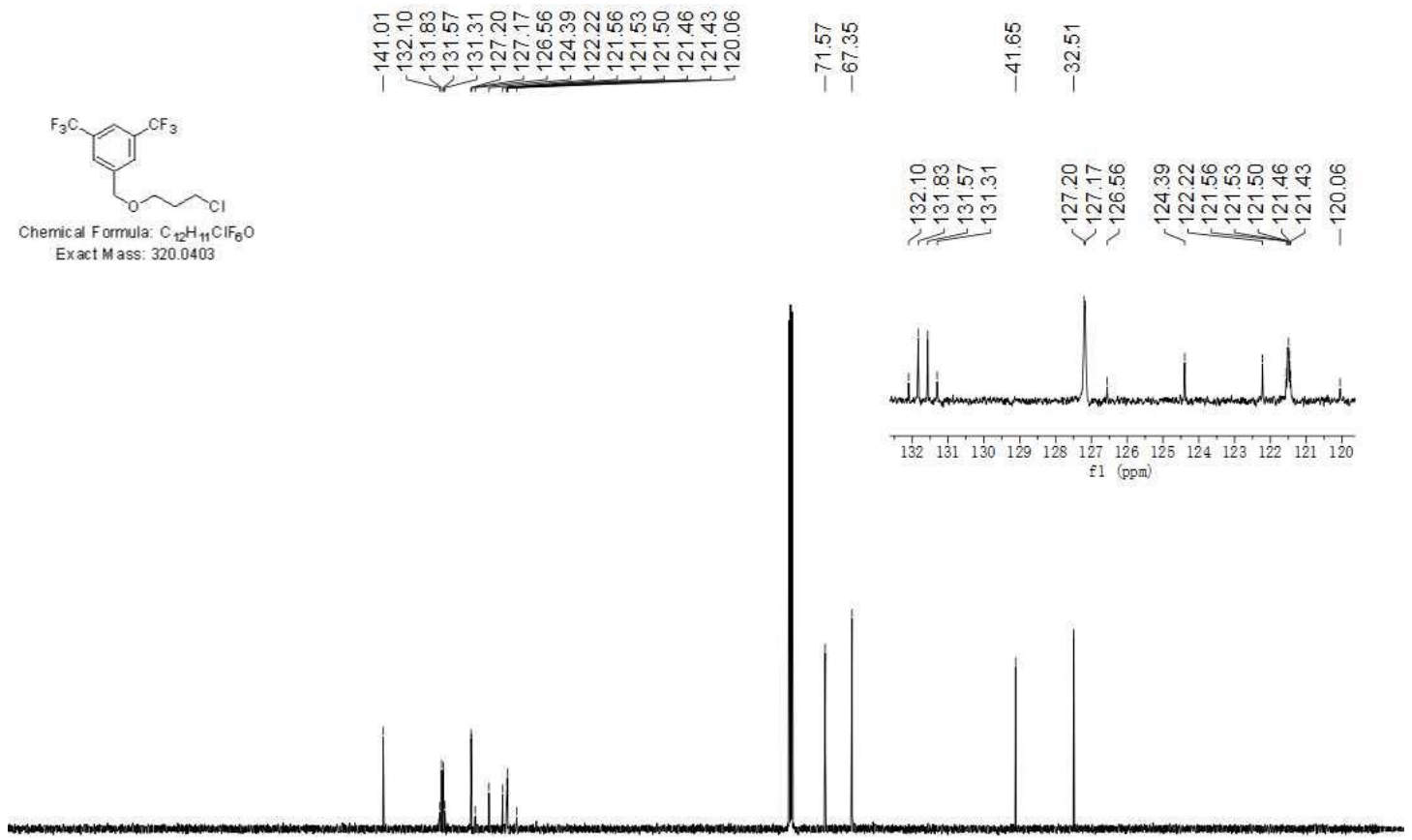

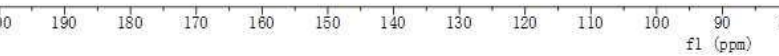


tert-Butyl (3,5-bis(trifluoromethyl)phenyl)carbamate (10)

${ }^{1} \mathrm{H}$ NMR of 10

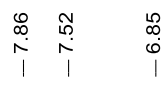

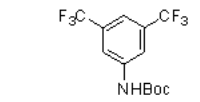

Chemical Formula: $\mathrm{C}_{13} \mathrm{H}_{13} \mathrm{~F}_{6} \mathrm{NO}_{2}$

Exact Mass: 329.0850

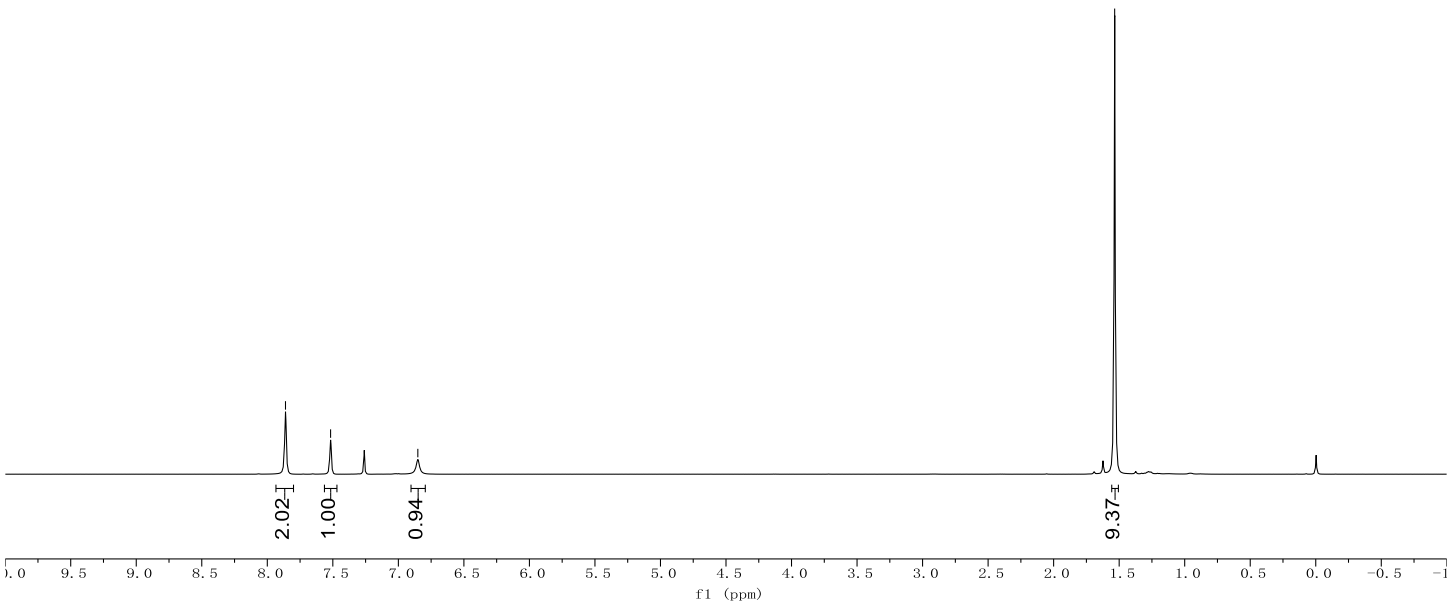

${ }^{19} \mathrm{~F}$ NMR of 10

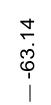

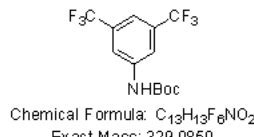

Exact

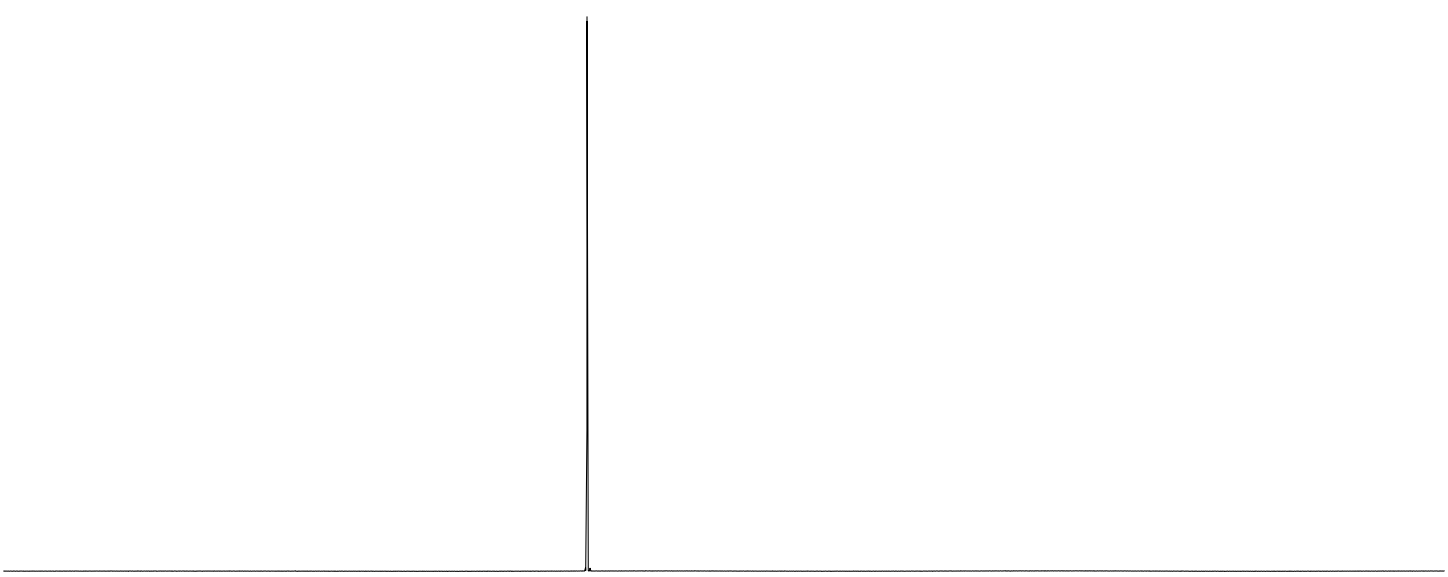

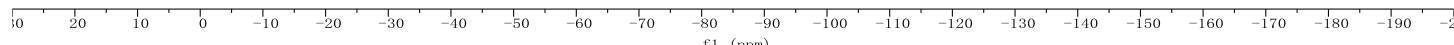


${ }^{13} \mathrm{C}$ NMR of 10

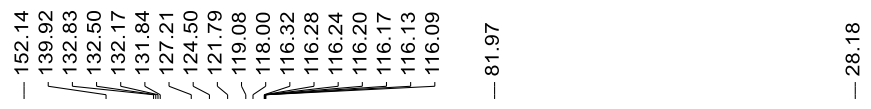
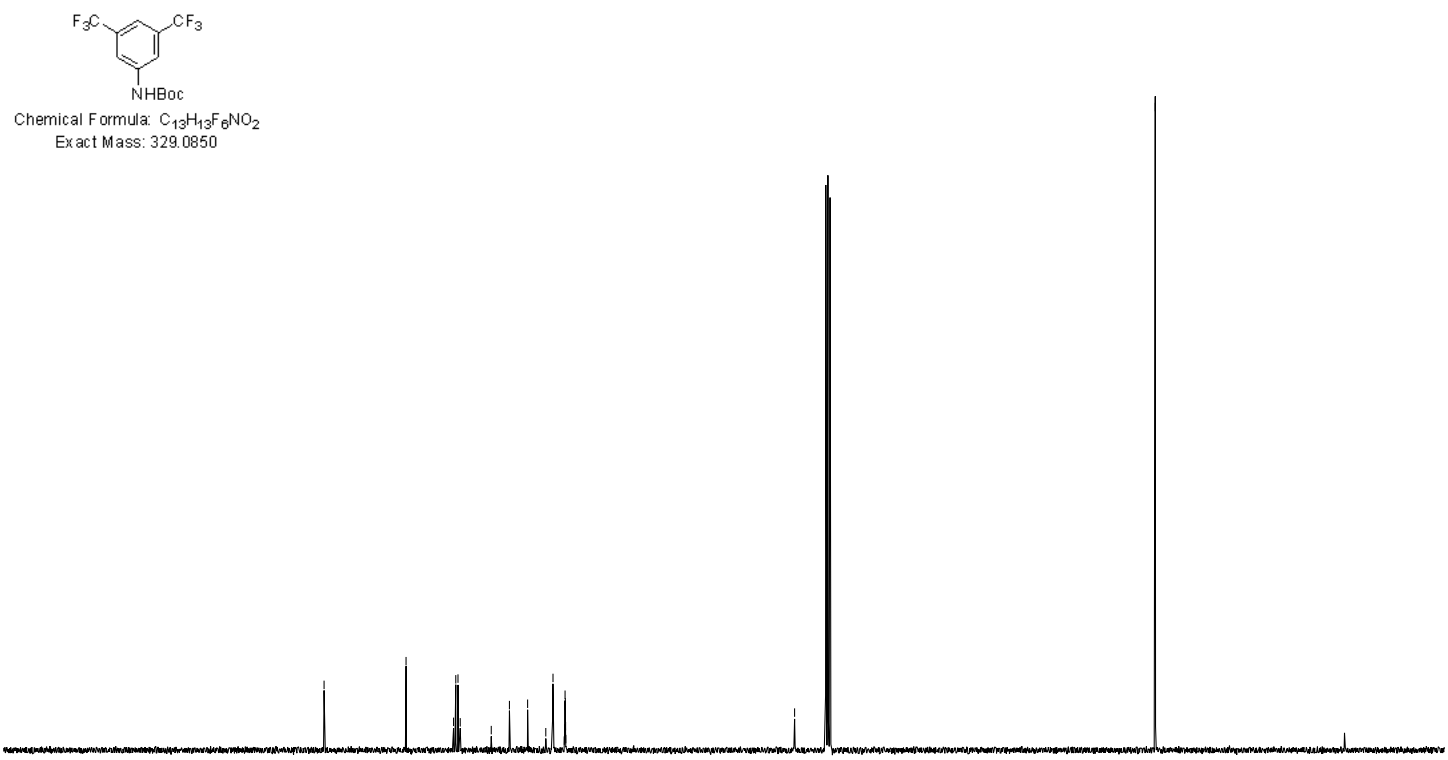

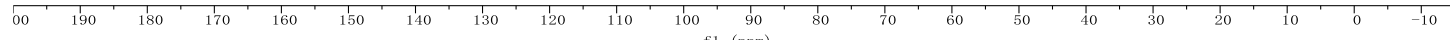

\section{$N$-Methyl- $N$-phenyl-3,5-bis(trifluoromethyl)aniline (1p)}

${ }^{1} \mathrm{H}$ NMR of $\mathbf{1 p}$
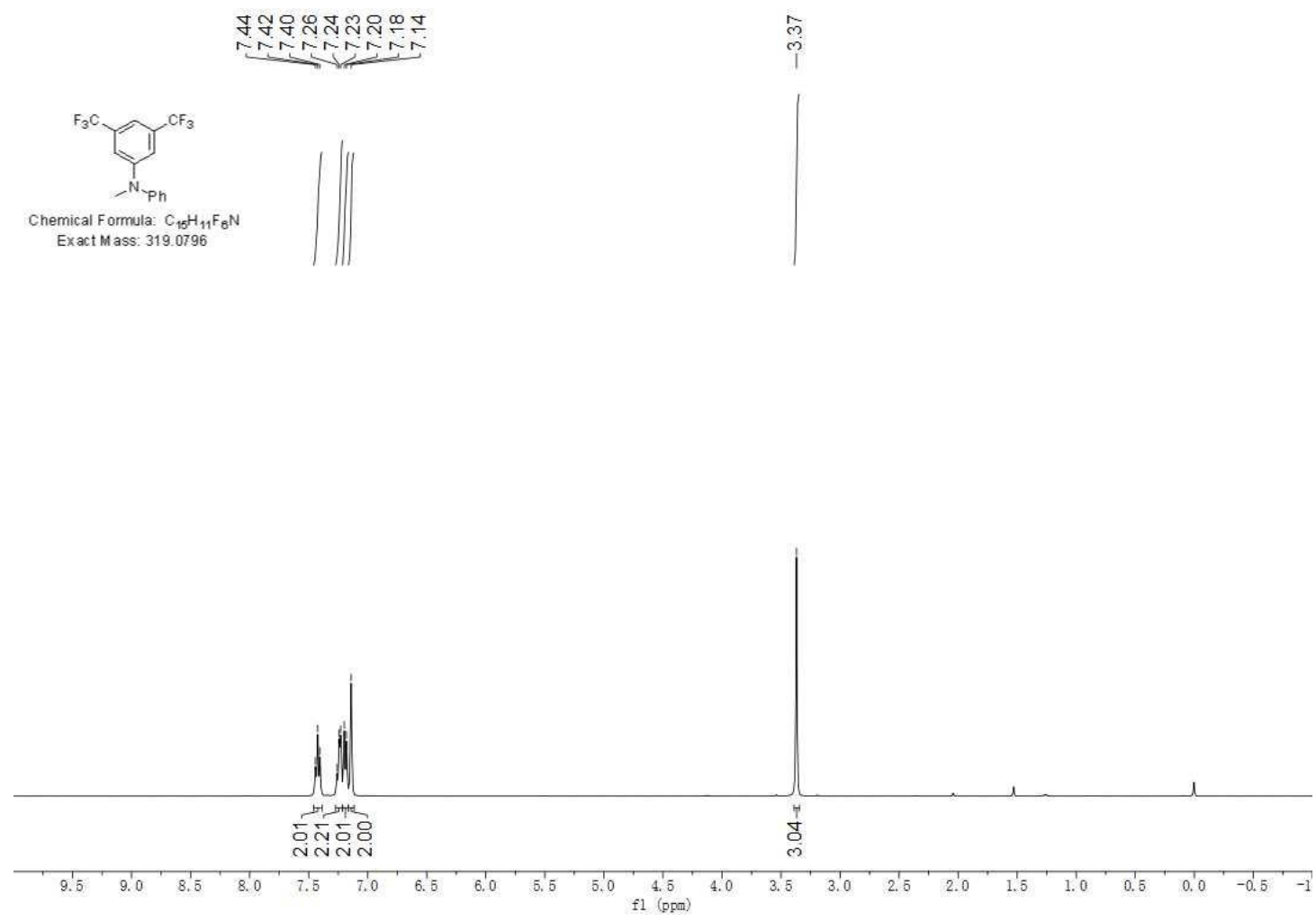
${ }^{19}$ F NMR of $1 p$

$\stackrel{\infty}{\oplus}$

$\mathrm{CF}_{3}$

Chemical Formula: $\mathrm{C}_{15} \mathrm{H}_{11} \mathrm{~F}_{8} \mathrm{~N}$
Exact Mass: 319.0796

(1)

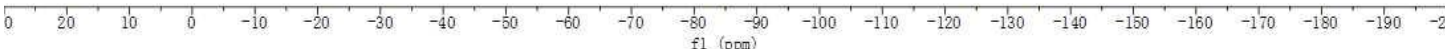

\section{${ }^{13} \mathrm{C}$ NMR of $\mathbf{1 p}$}

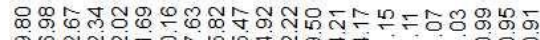

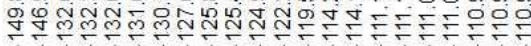

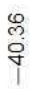

chemical Formula: $\mathrm{C}_{15} \mathrm{H}_{11} \mathrm{~F}_{6} \mathrm{~N}$

फิ

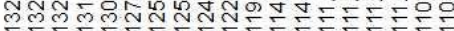

Exact Mass: 3190796
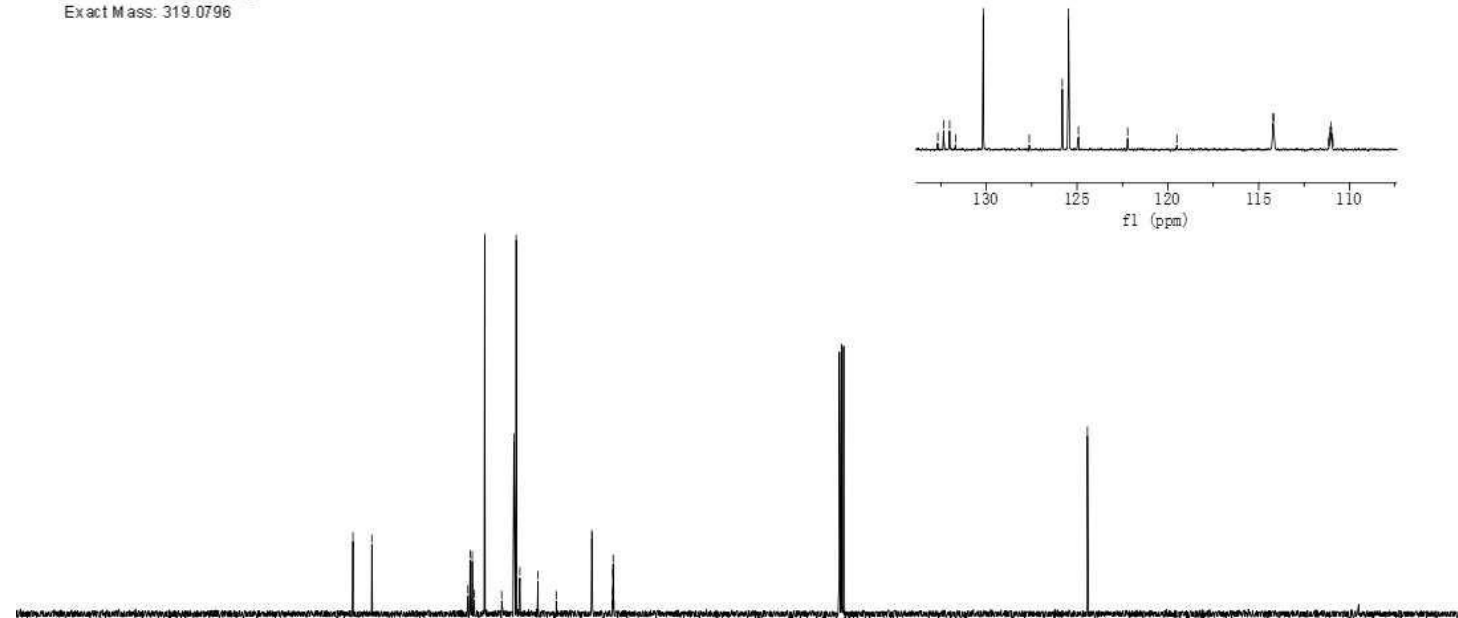

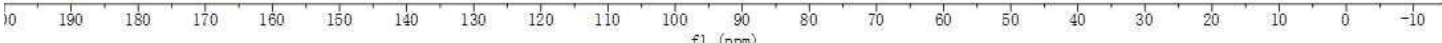


3,5-Bis(trifluoromethyl)-1,1'-biphenyl (1q)

${ }^{1} \mathrm{H}$ NMR of $\mathbf{1 q}$
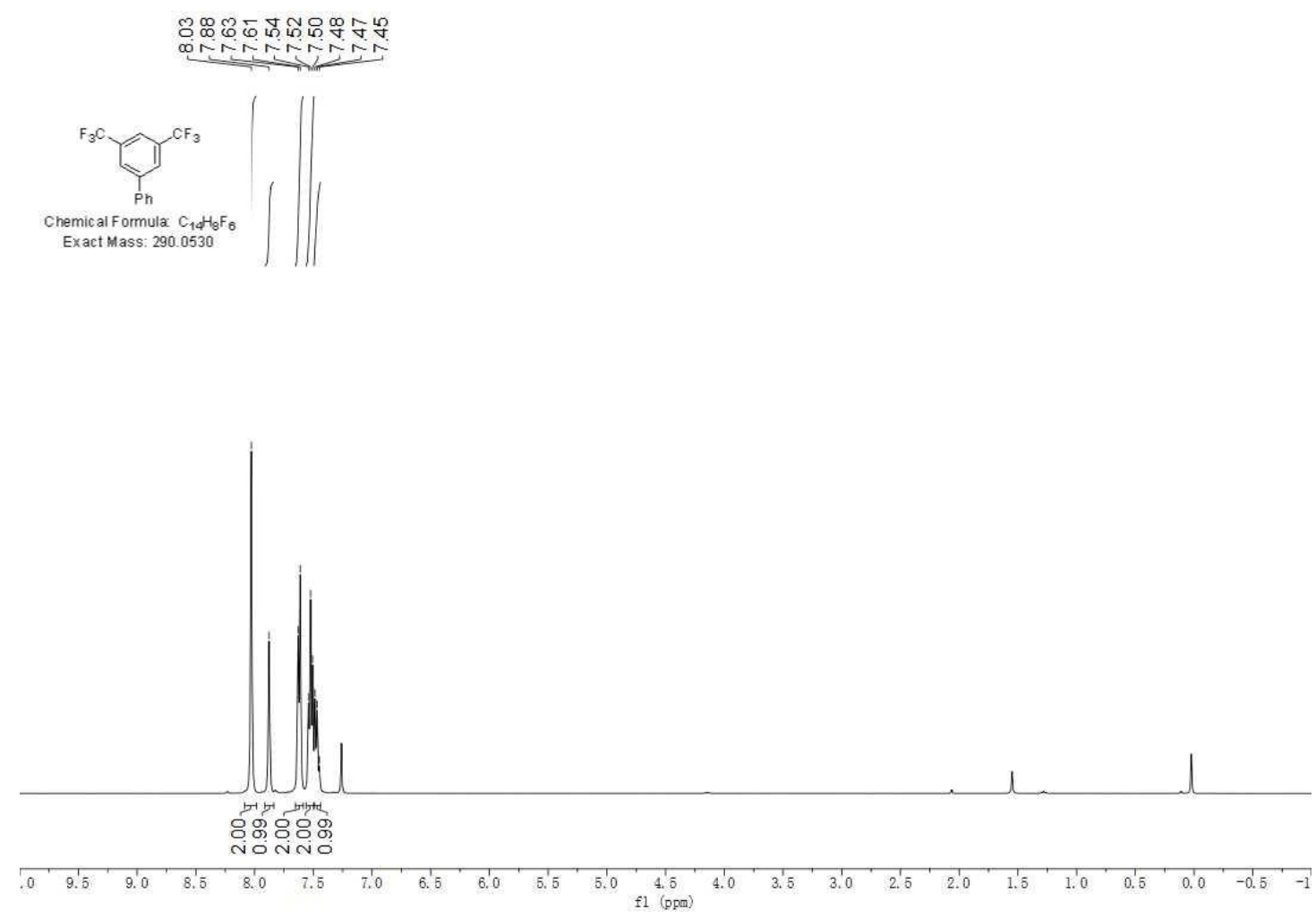

${ }^{19}$ F NMR of 1q

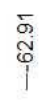
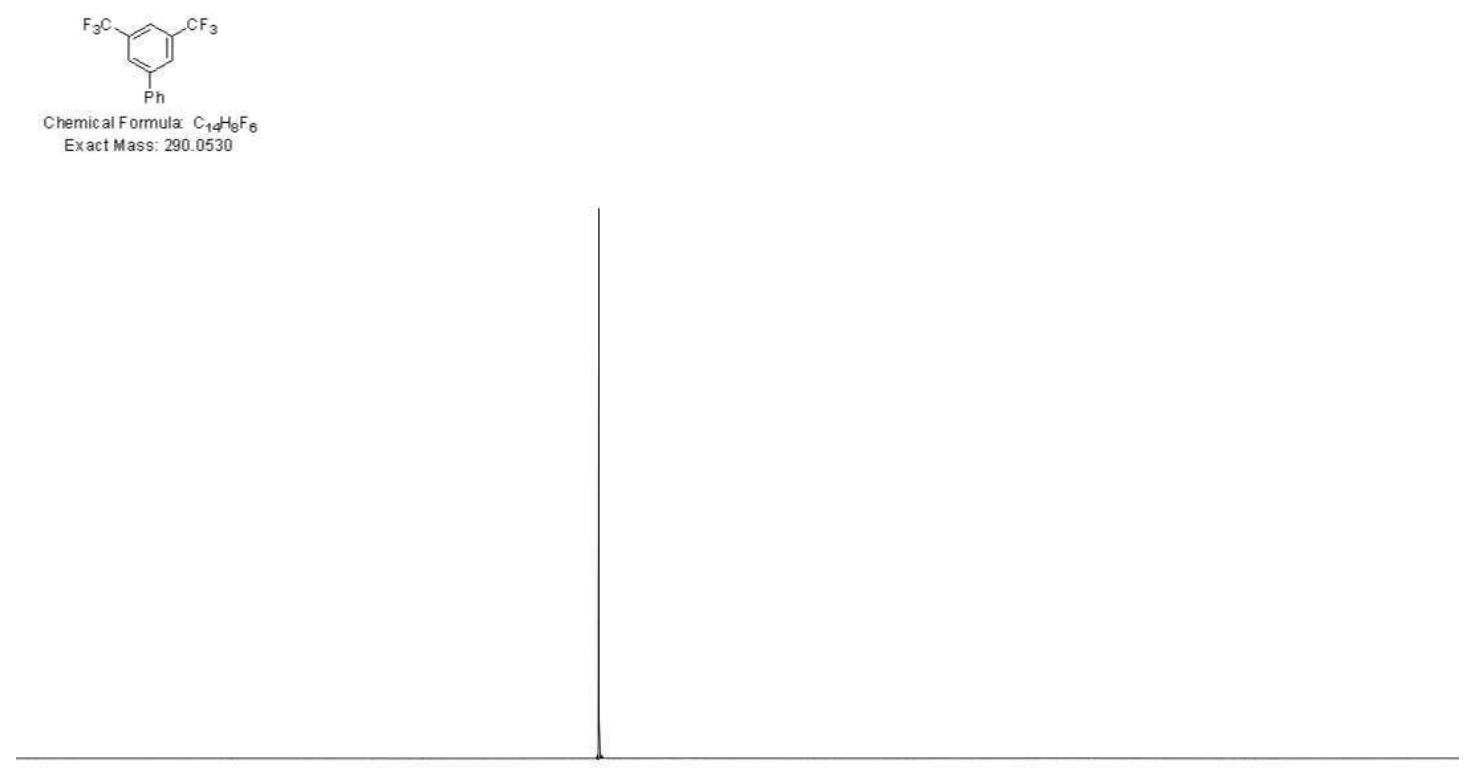

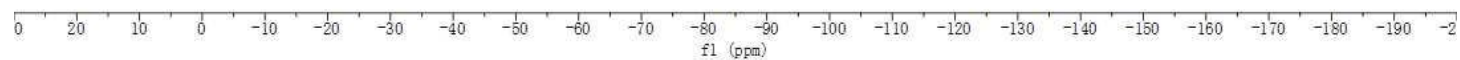


${ }^{13} \mathrm{C}$ NMR of 1q

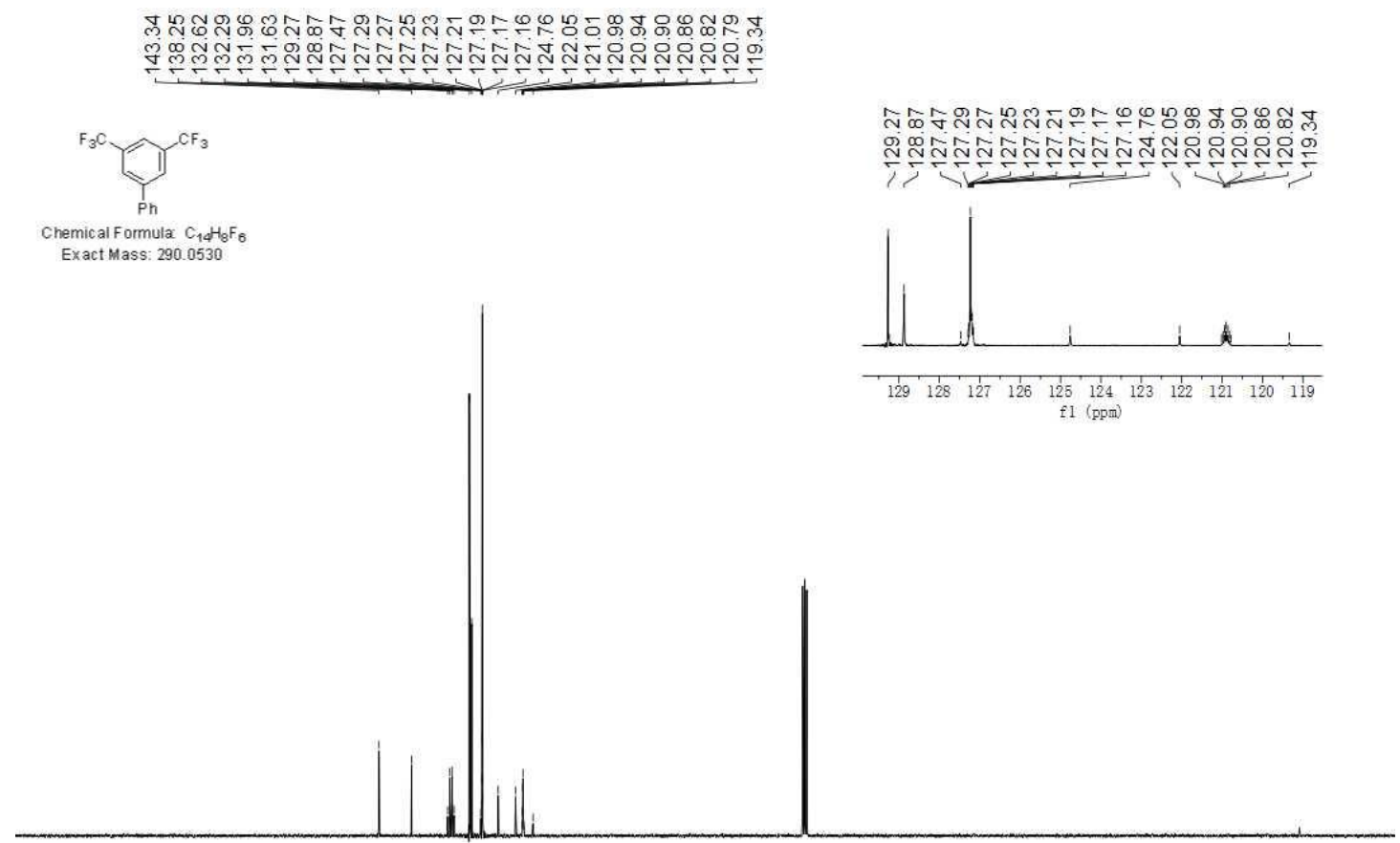

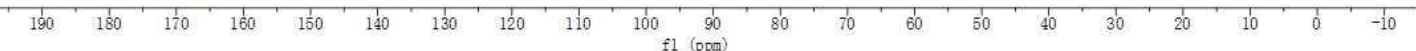

\section{Morpholino(3-(trifluoromethyl)phenyl)methanone (1u)}

${ }^{1} \mathrm{H}$ NMR of $\mathbf{1 u}$

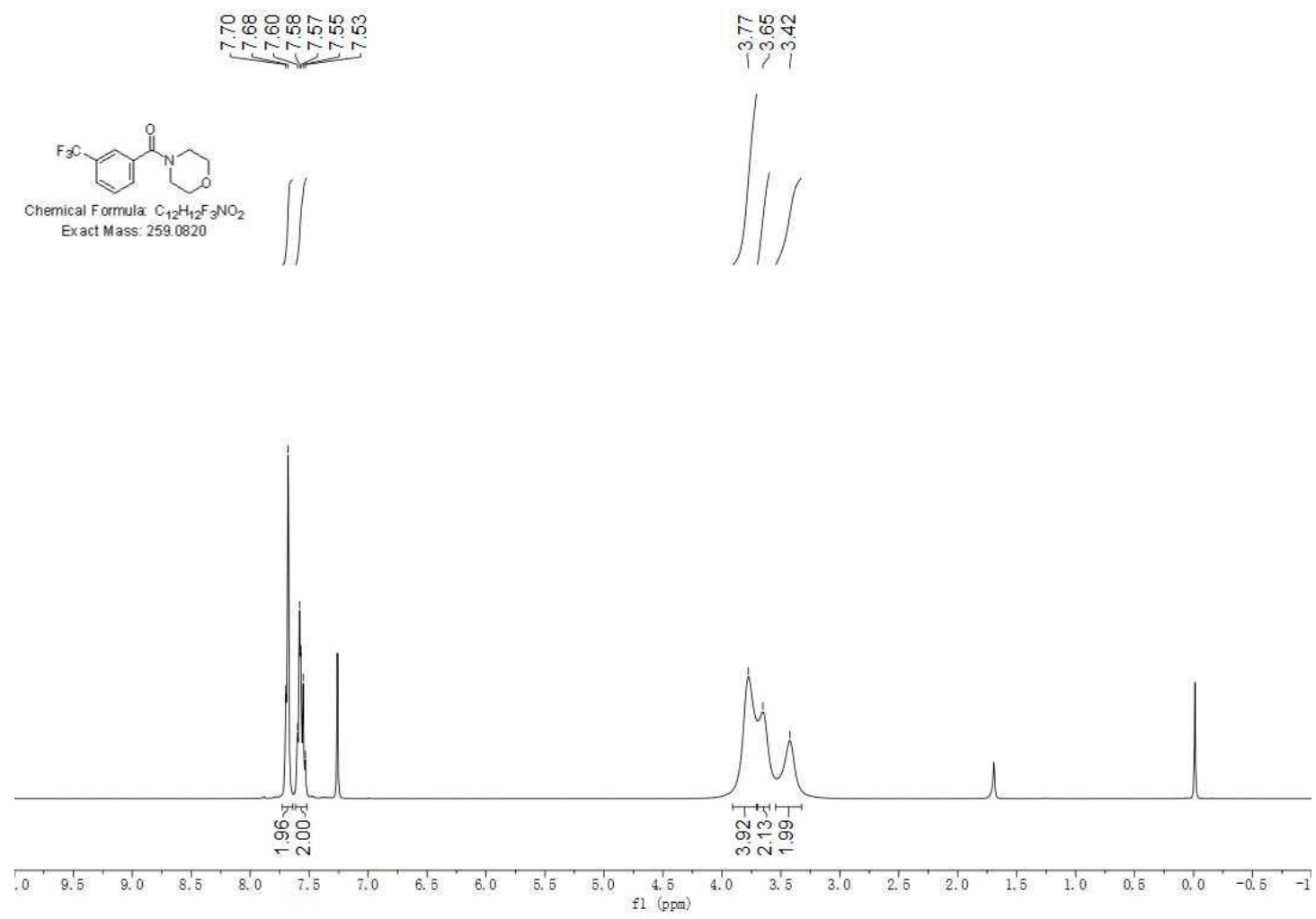


${ }^{19} \mathrm{~F}$ NMR of $\mathbf{1 u}$

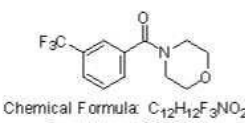

Exact Mass: 2590820

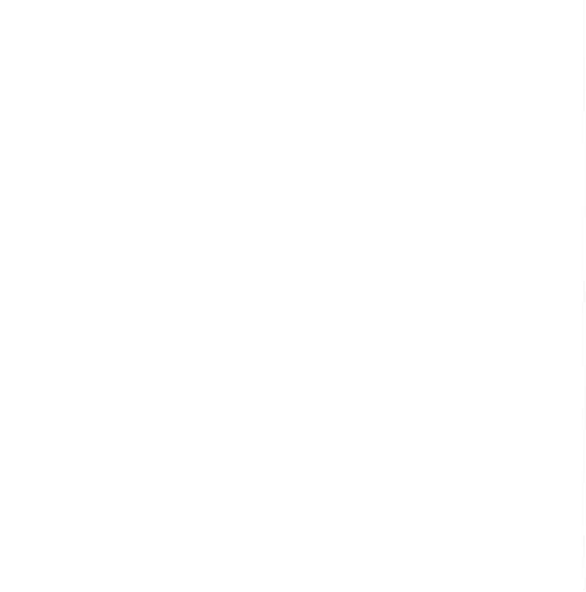

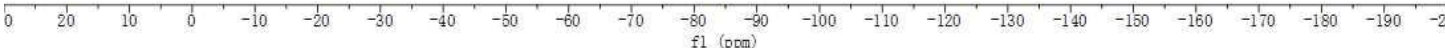

${ }^{13} \mathrm{C}$ NMR of $\mathbf{1 u}$

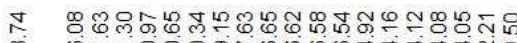

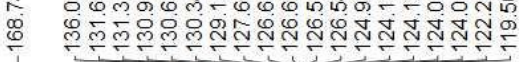

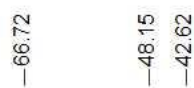

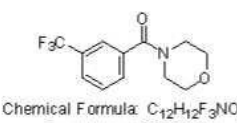

Exact Mass: 259.0920

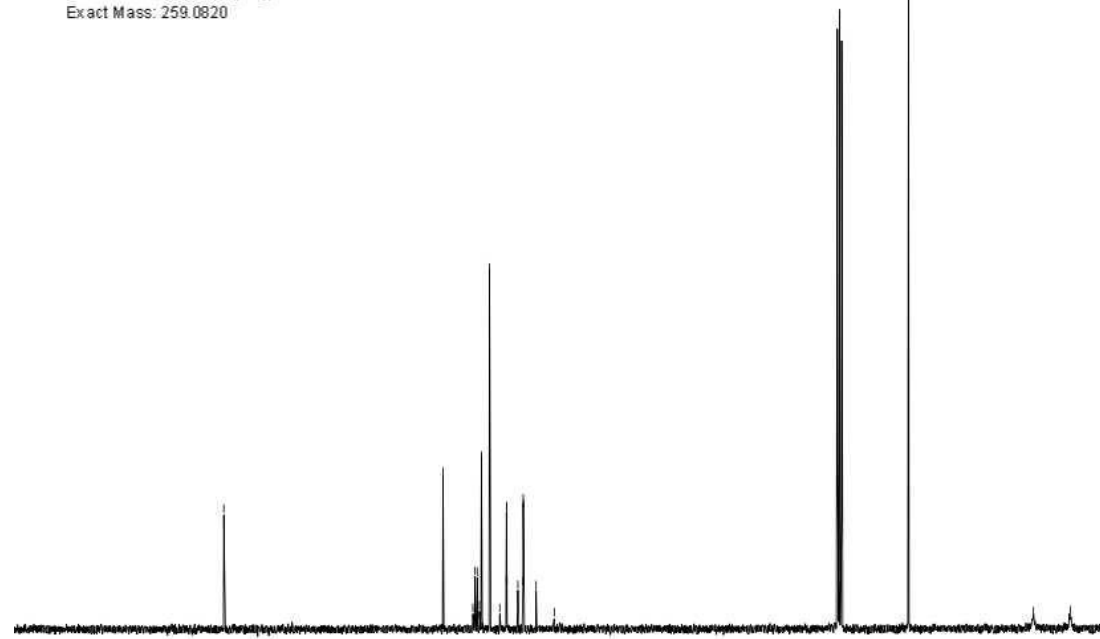

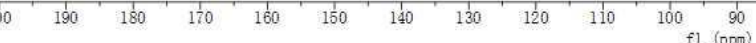

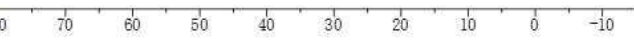


$N, N$-Diethyl-3-(trifluoromethyl)benzenesulfonamide (1v)

${ }^{1} \mathrm{H}$ NMR of $1 \mathbf{v}$

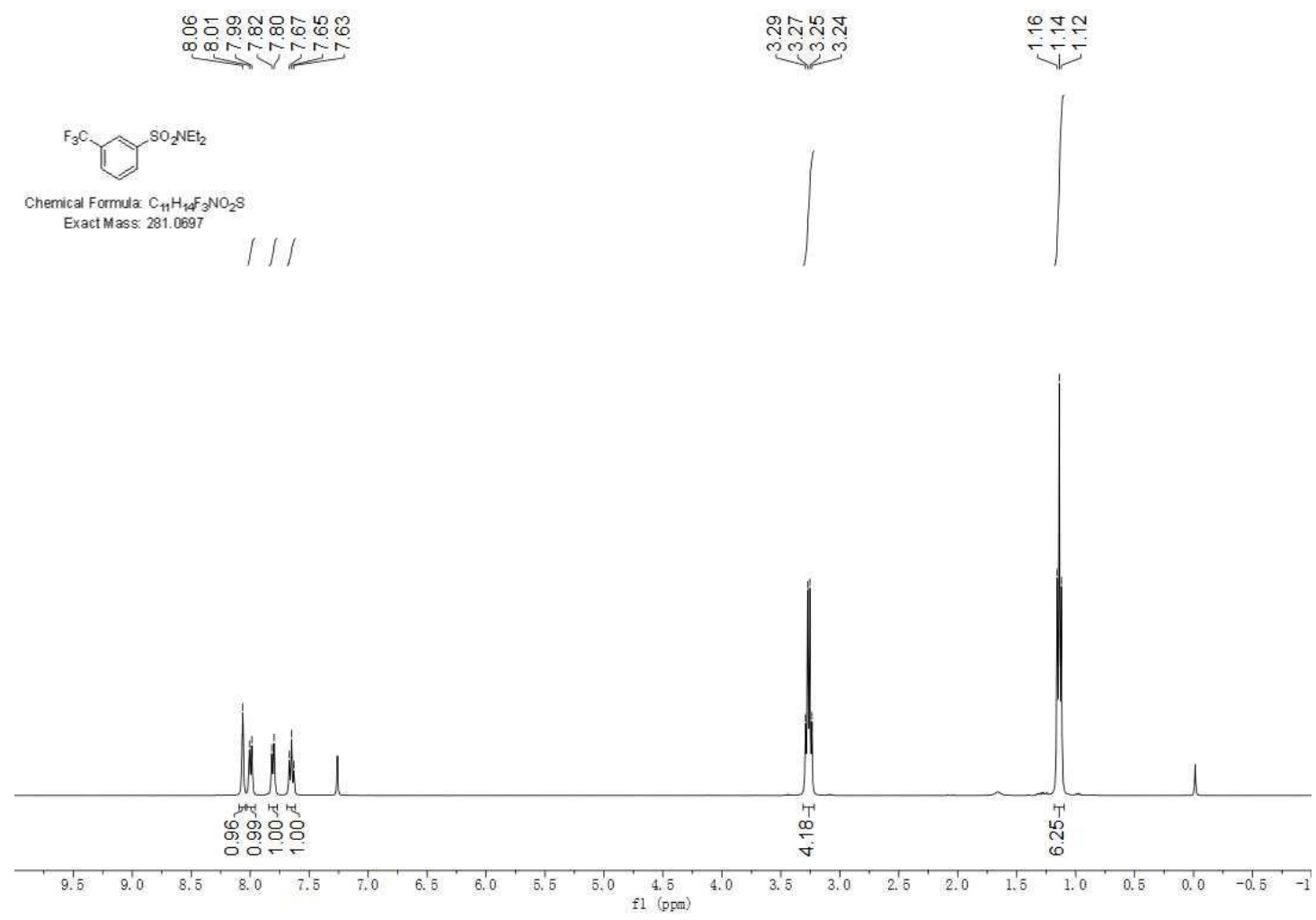

${ }^{19} \mathrm{~F}$ NMR of $\mathbf{1 v}$

9
$\infty$
$\mathbb{1}$
1

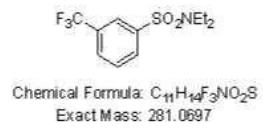

Exact Mass: 281.0697

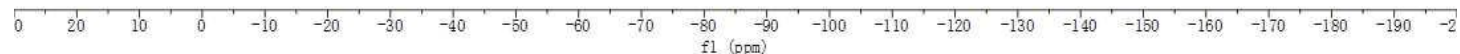


${ }^{13} \mathrm{C}$ NMR of $\mathbf{1 v}$

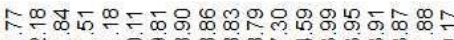

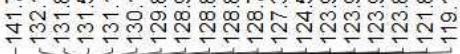

กั

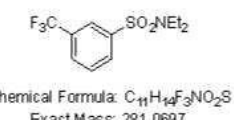

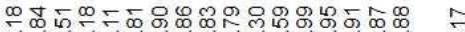

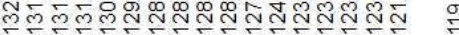

Exact Mass. 281.0697
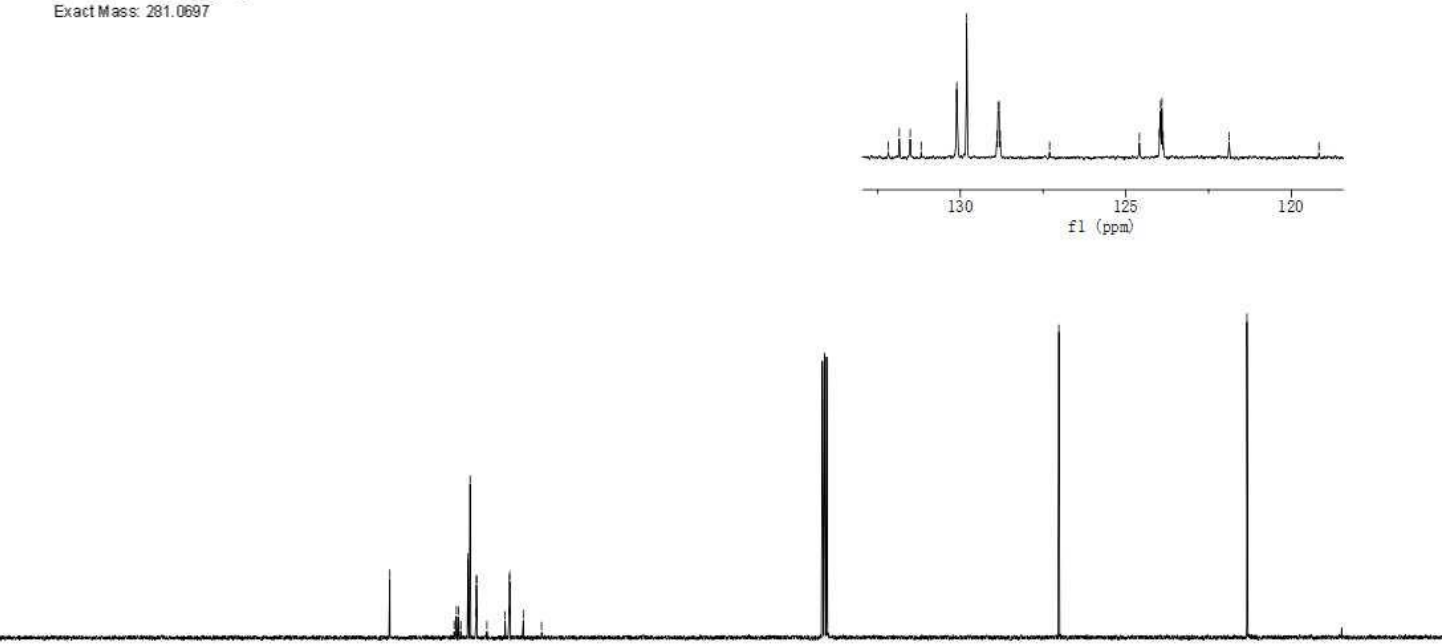

$\begin{array}{lllllllllll}190 & 180 & 170 & 160 & 150 & 140 & 130 & 120 & 110 & 100 & 90\end{array}$

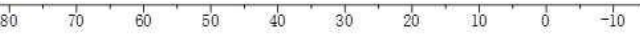

\section{1-(Difluoro(phenyl)methyl)-3-(trifluoromethyl)benzene (3a)}

${ }^{1} \mathrm{H}$ NMR of 3a

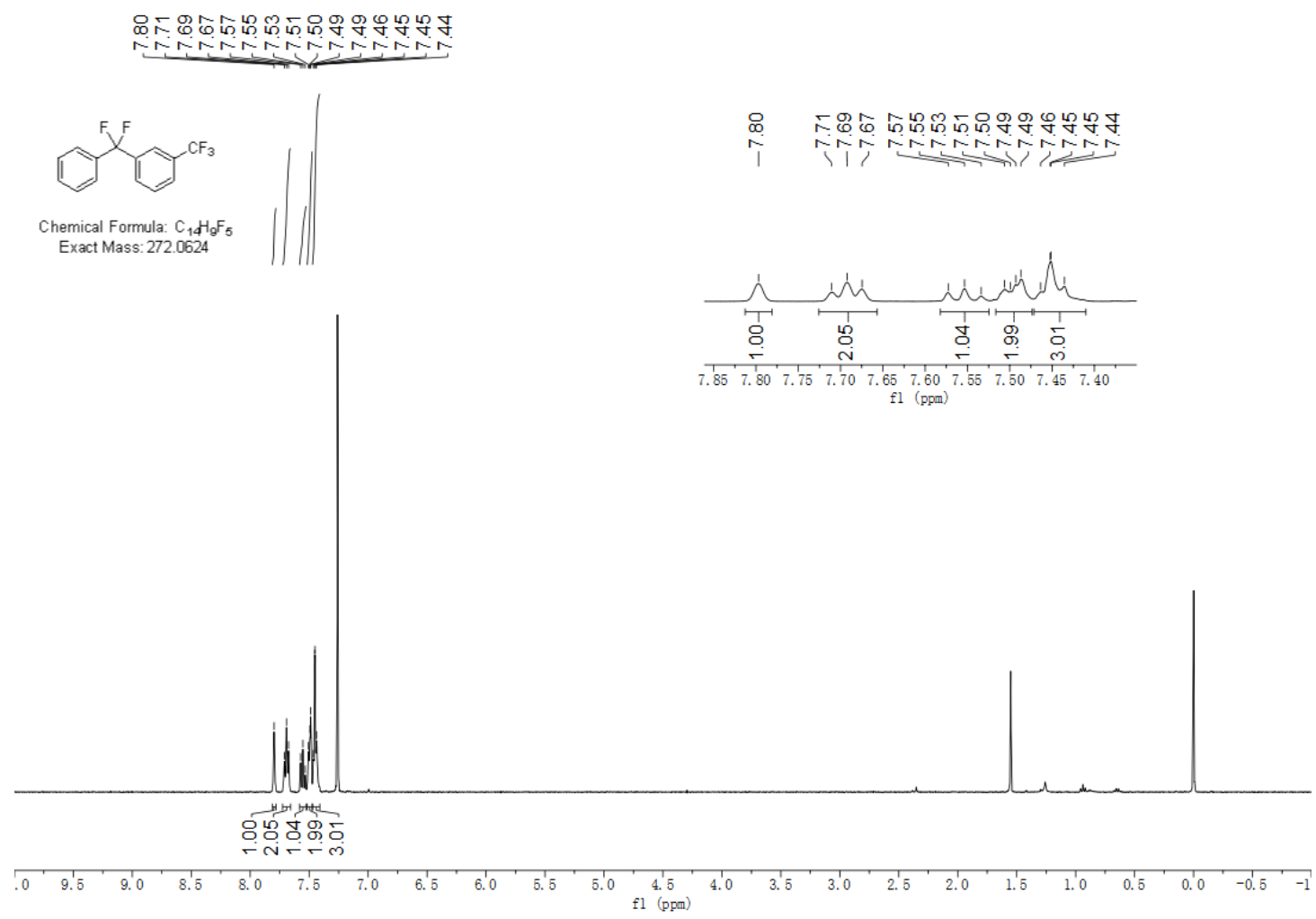


${ }^{19} \mathrm{~F}$ NMR of $\mathbf{3 a}$

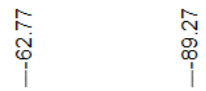

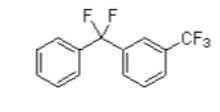

Chemical Formula: $\mathrm{C}_{14} \mathrm{H}_{9} \mathrm{~F}_{5}$

Exact Mass: 272.0624

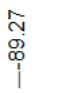

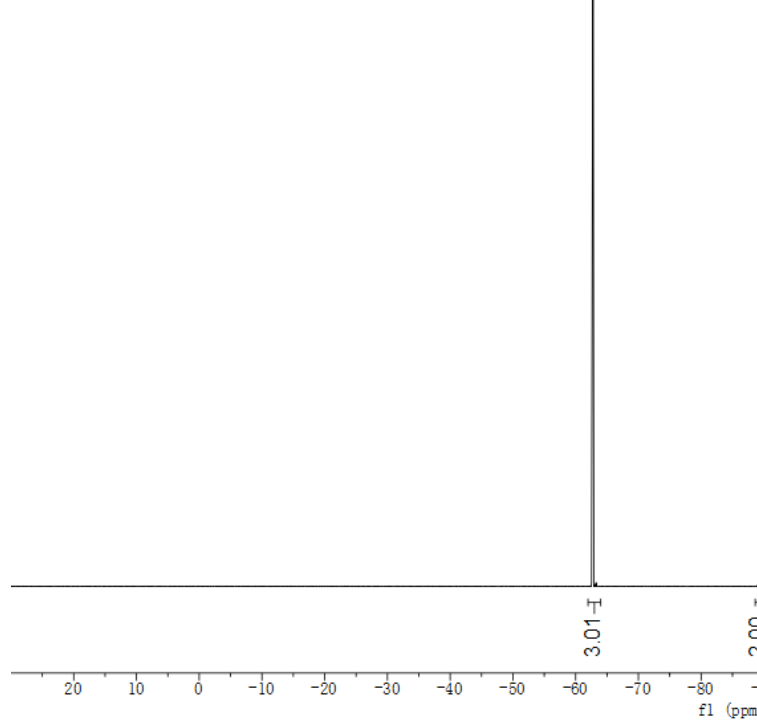

\section{${ }^{13} \mathrm{C}$ NMR of $3 \mathbf{a}$}

\%드유.

on
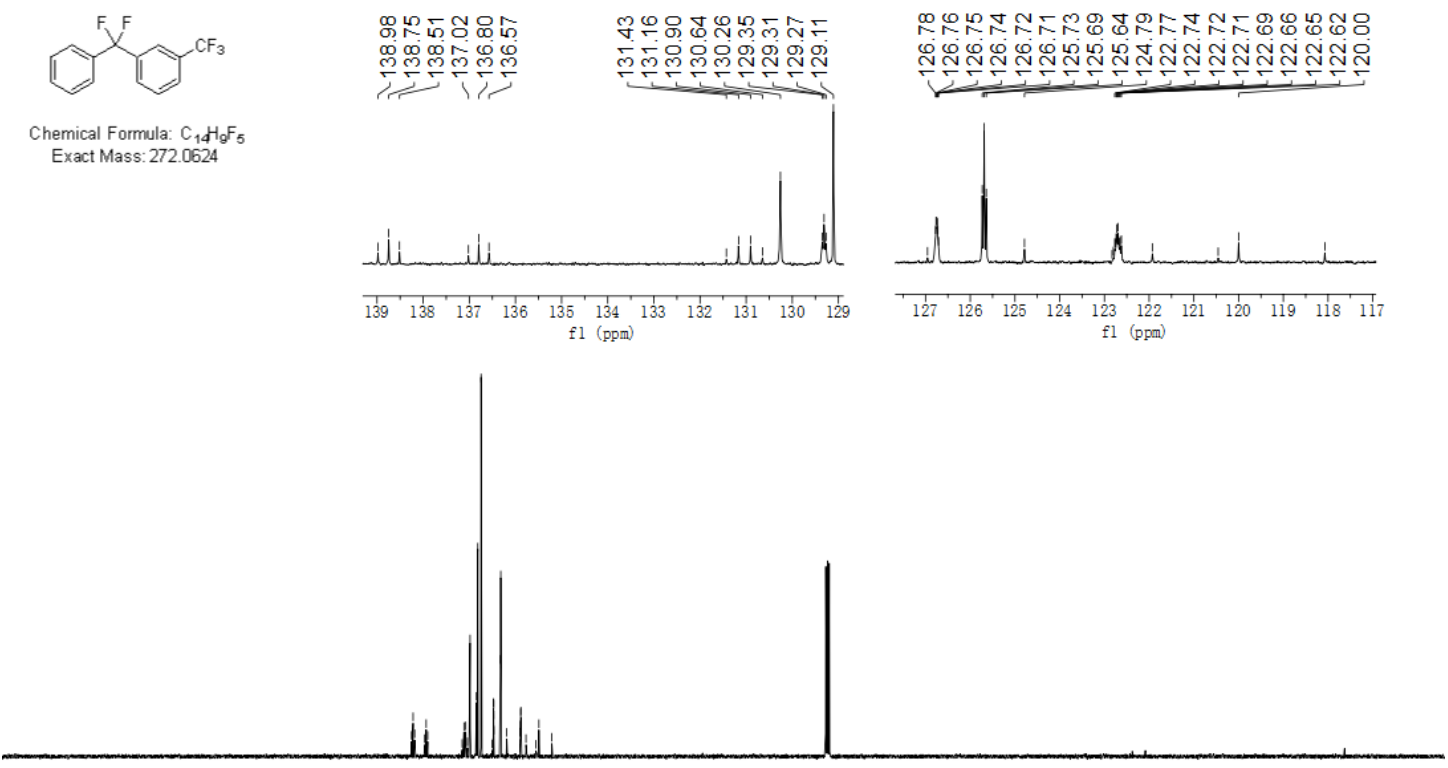

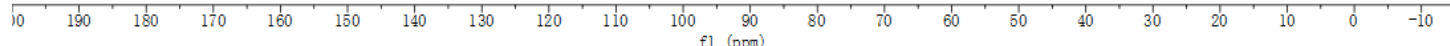


1,2-Difluoro-1,2-diphenyl-1,2-bis(3-(trifluoromethyl)phenyl)ethane (5a).

${ }^{1} \mathrm{H}$ NMR of $\mathbf{5 a}$

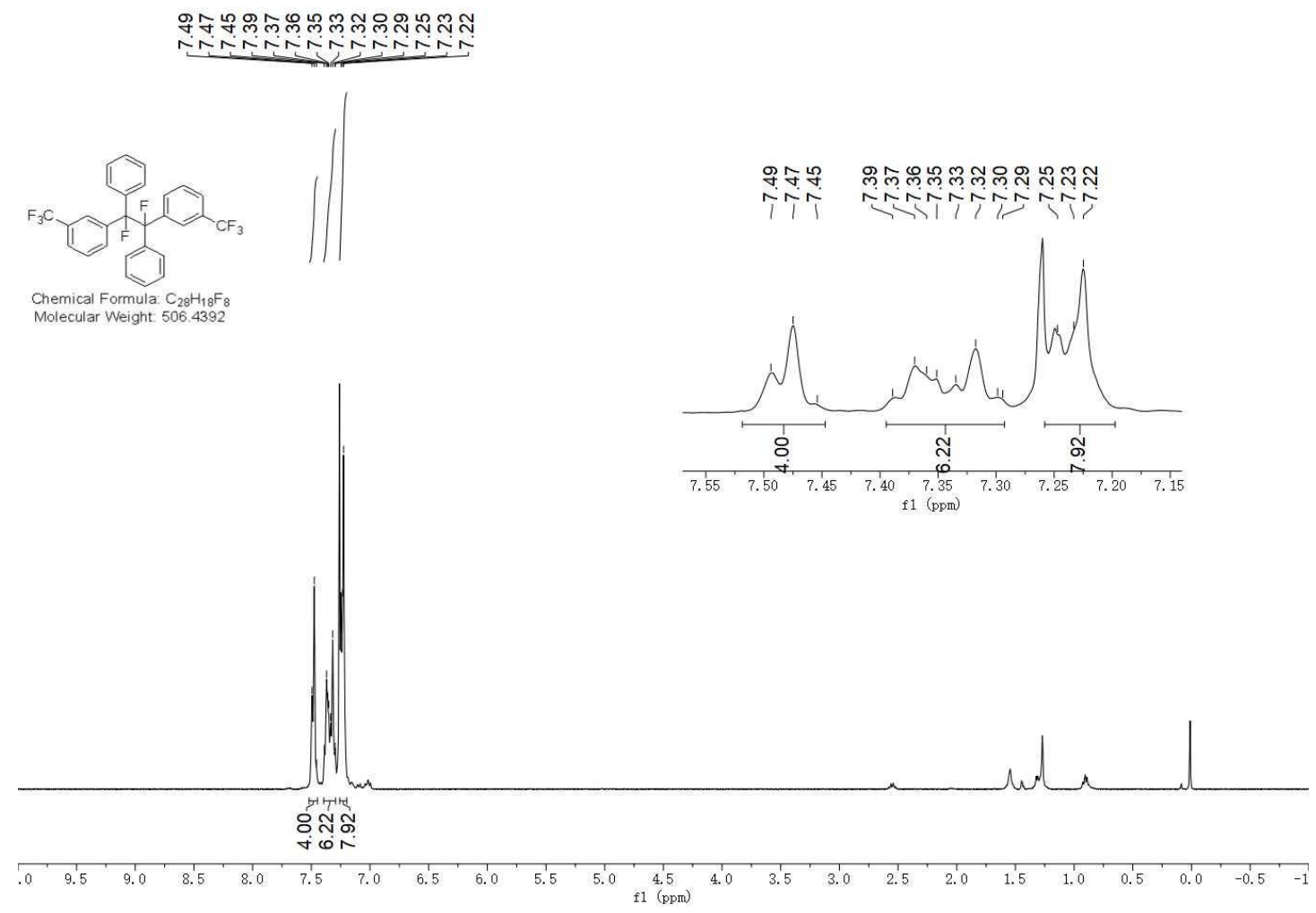

${ }^{19} \mathrm{~F}$ NMR of $\mathbf{5 a}$

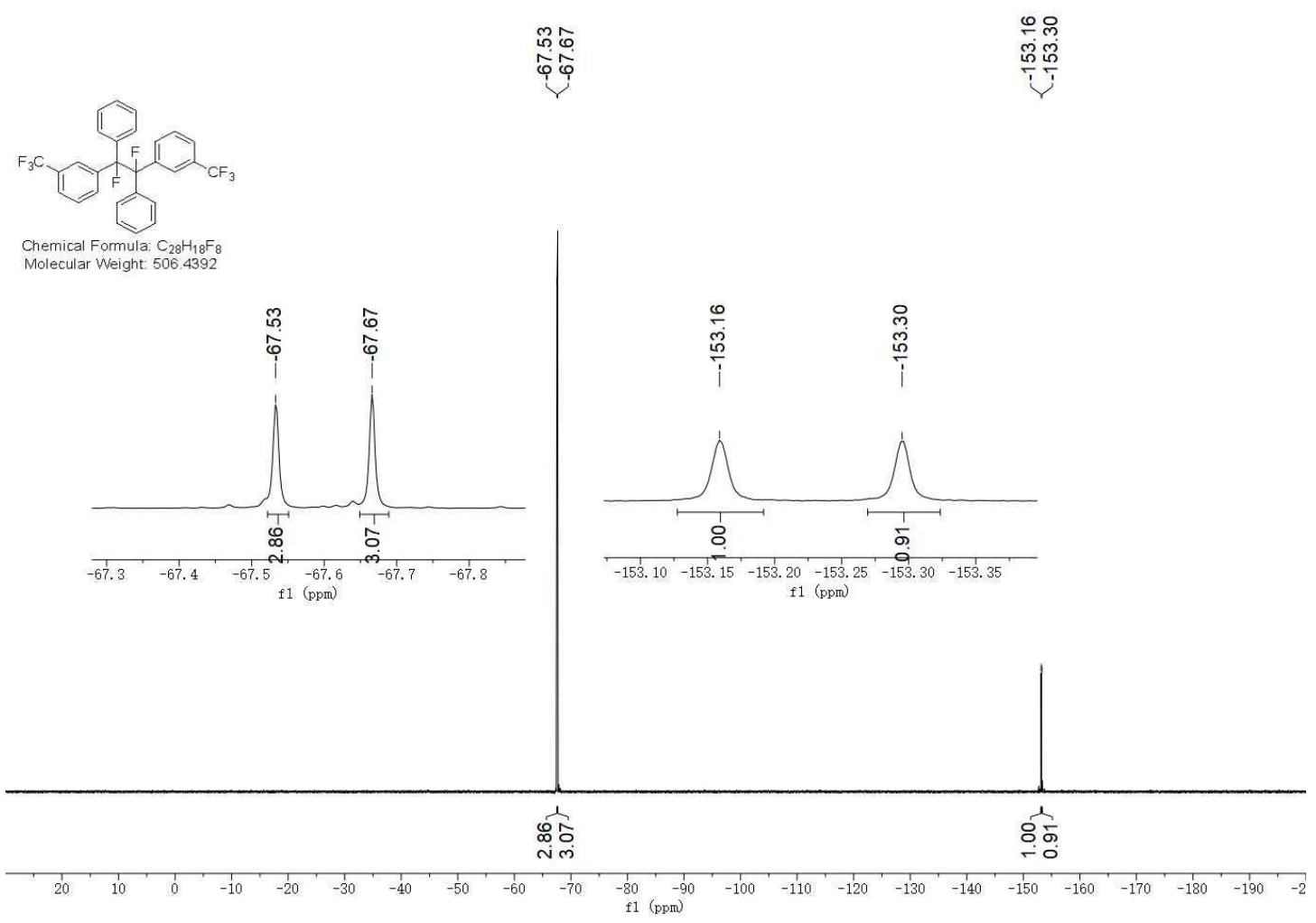


1-(Difluoro(3-(trifluoromethyl)phenyl)methyl)-2-methylbenzene (3b)

${ }^{1} \mathrm{H}$ NMR of $\mathbf{3 b}$

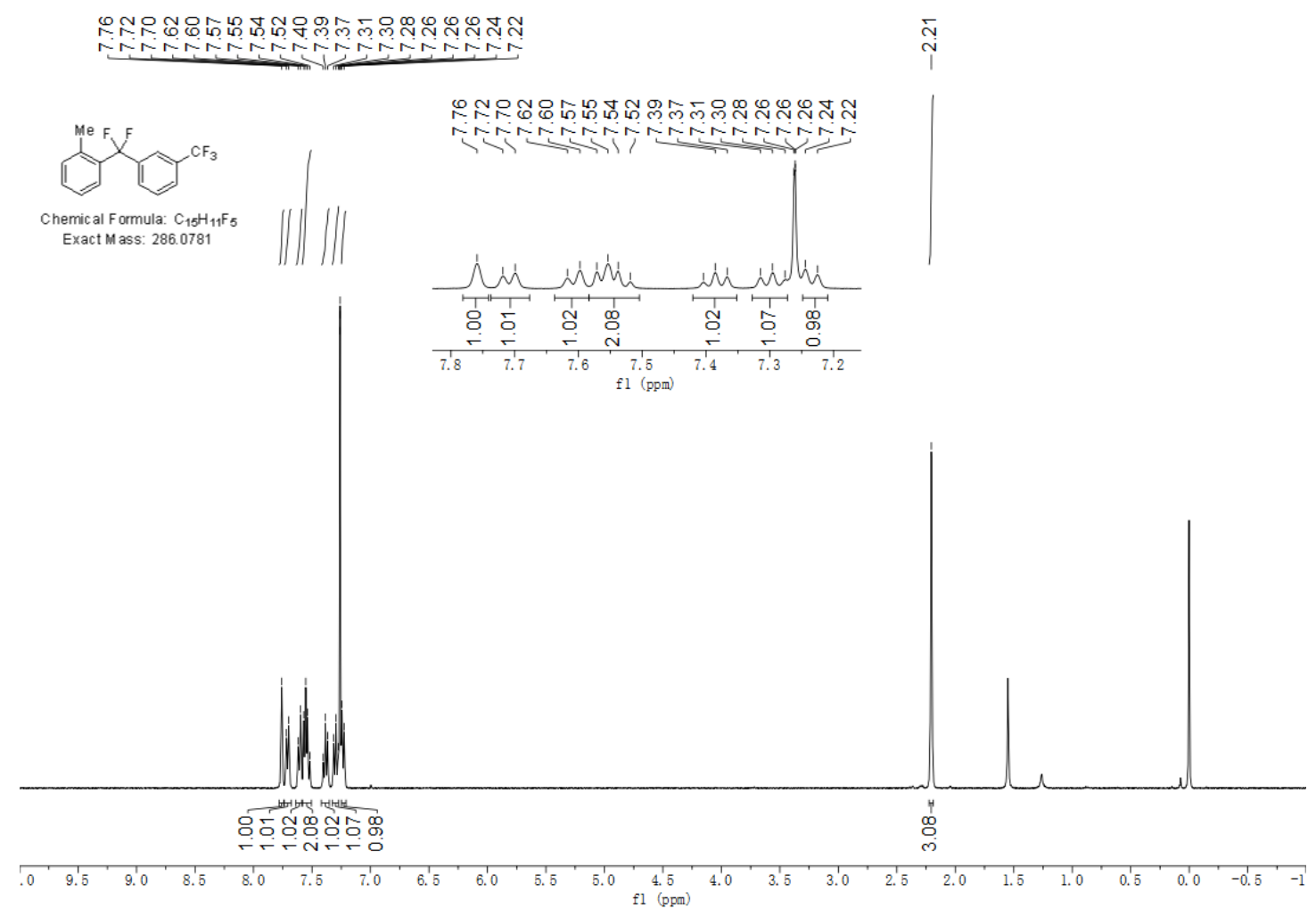

${ }^{19} \mathrm{~F}$ NMR of $\mathbf{3 b}$

$\underset{\substack{0 \\ 0}}{\stackrel{5}{c}}$
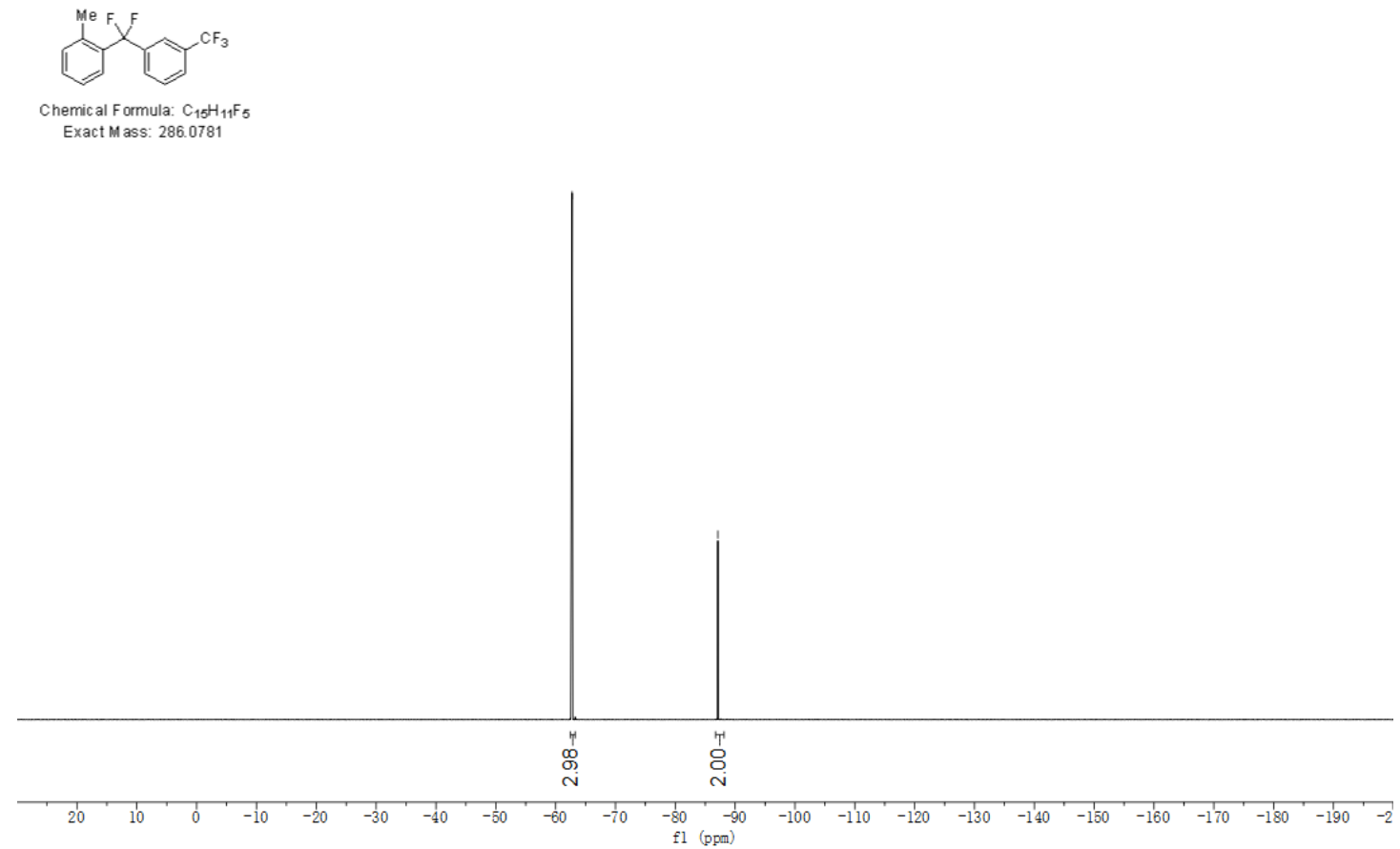
${ }^{13} \mathrm{C}$ NMR of $\mathbf{3 b}$

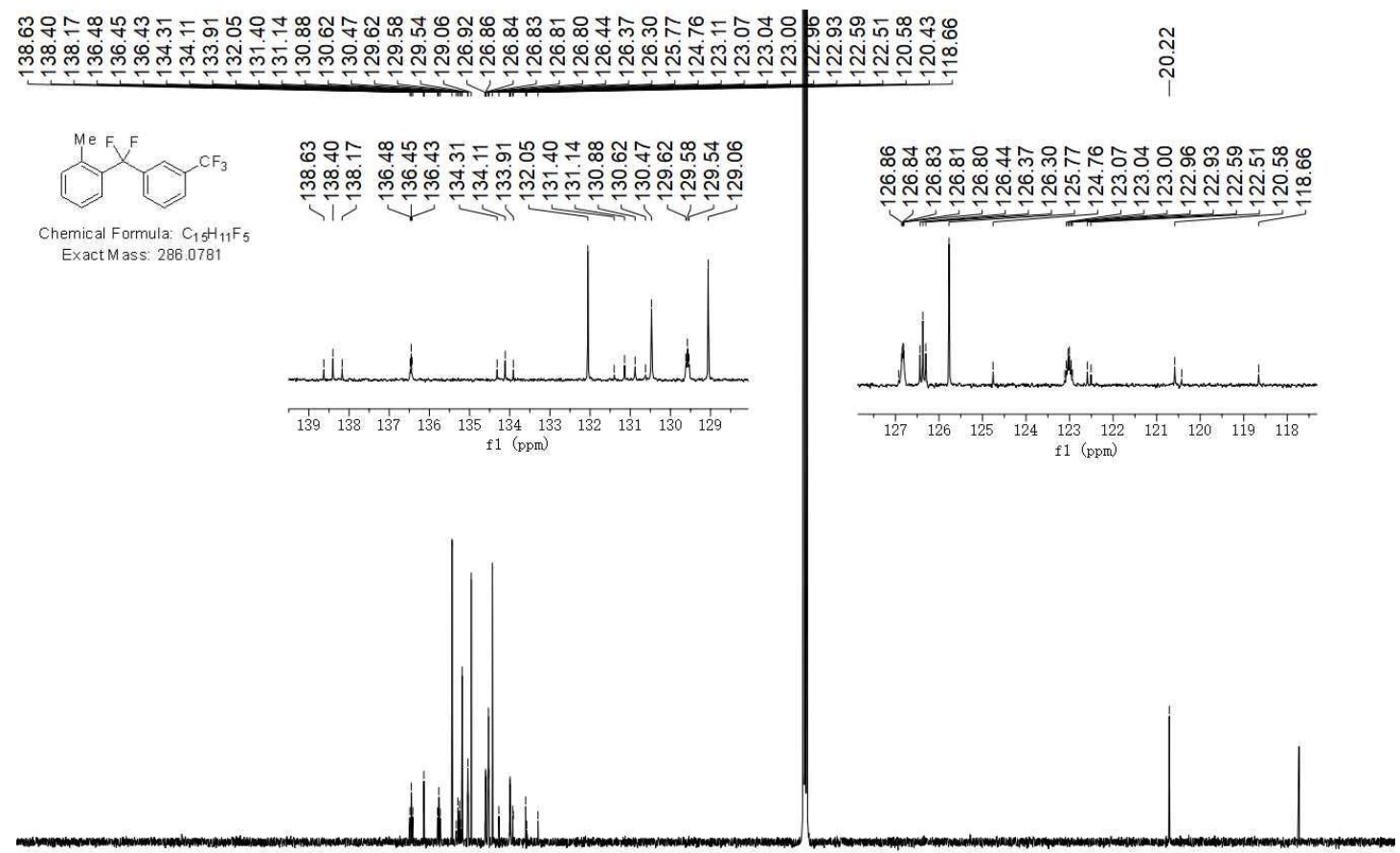

$10 \quad \begin{array}{lllllllllll}190 & 180 & 170 & 160 & 150 & 140 & 130 & 120 & 110 & 100 & 90 \\ \mathrm{f} 1 & & & & & & & & & & \end{array}$

1-(Difluoro(p-tolyl)methyl)-3-(trifluoromethyl)benzene (3c)

${ }^{1} \mathrm{H}$ NMR of $3 \mathrm{c}$

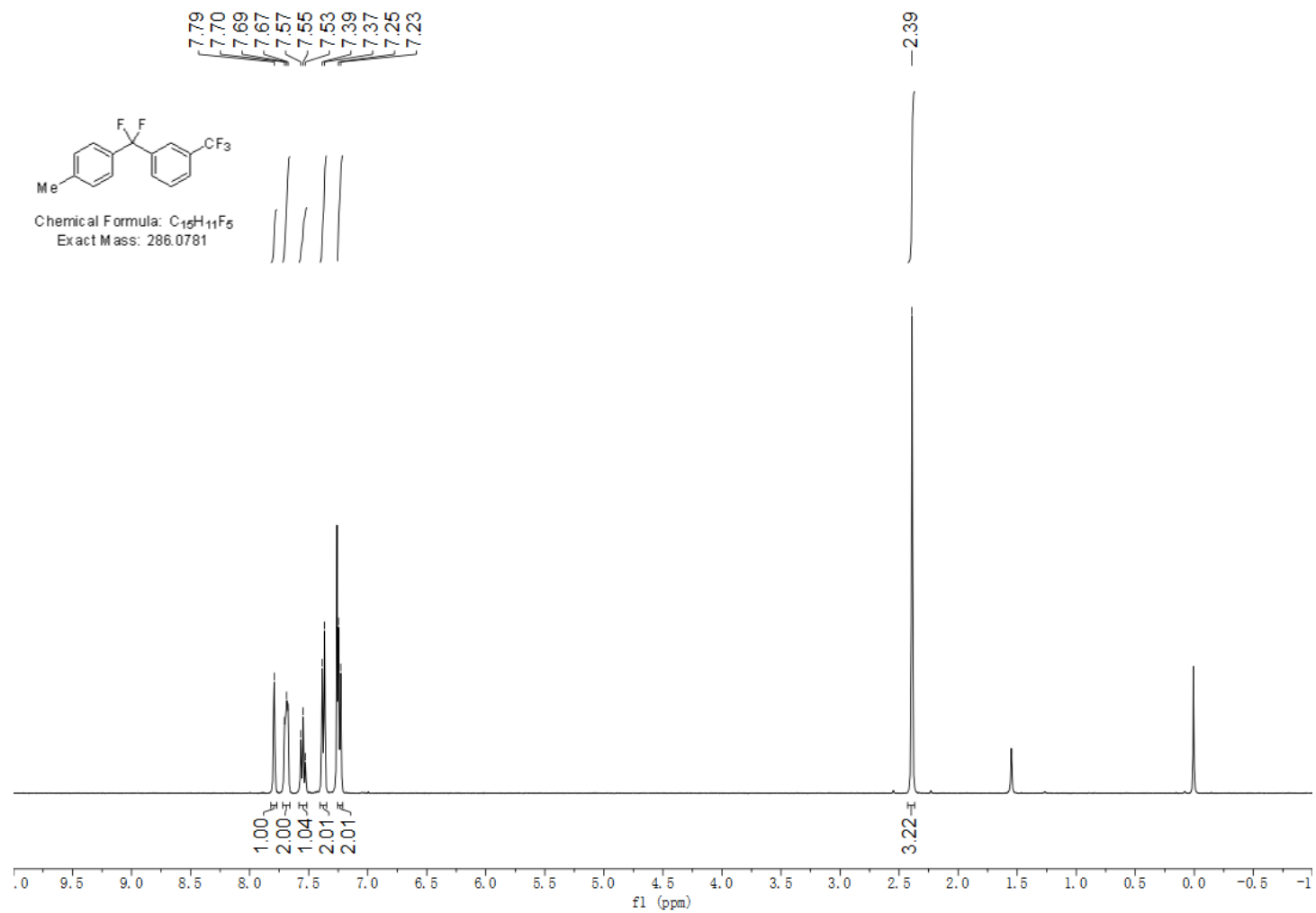


${ }^{19} \mathrm{~F}$ NMR of $\mathbf{3 c}$

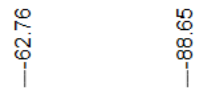

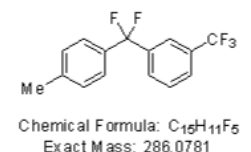

Exact Mass: 2860781

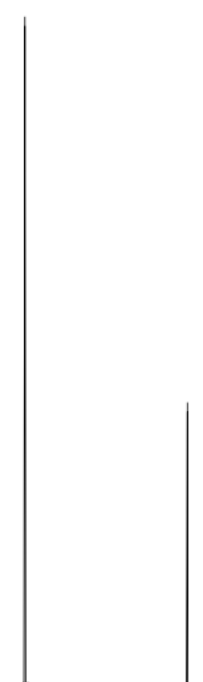

$\begin{array}{llllllllllll}20 & 10 & 0 & -10 & -20 & -10 & -40 & -50 & -60 & -10 & -80 & -90\end{array}$

\section{${ }^{13} \mathrm{C}$ NMR of $3 \mathrm{c}$}

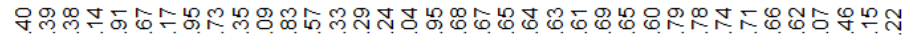

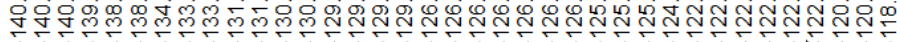

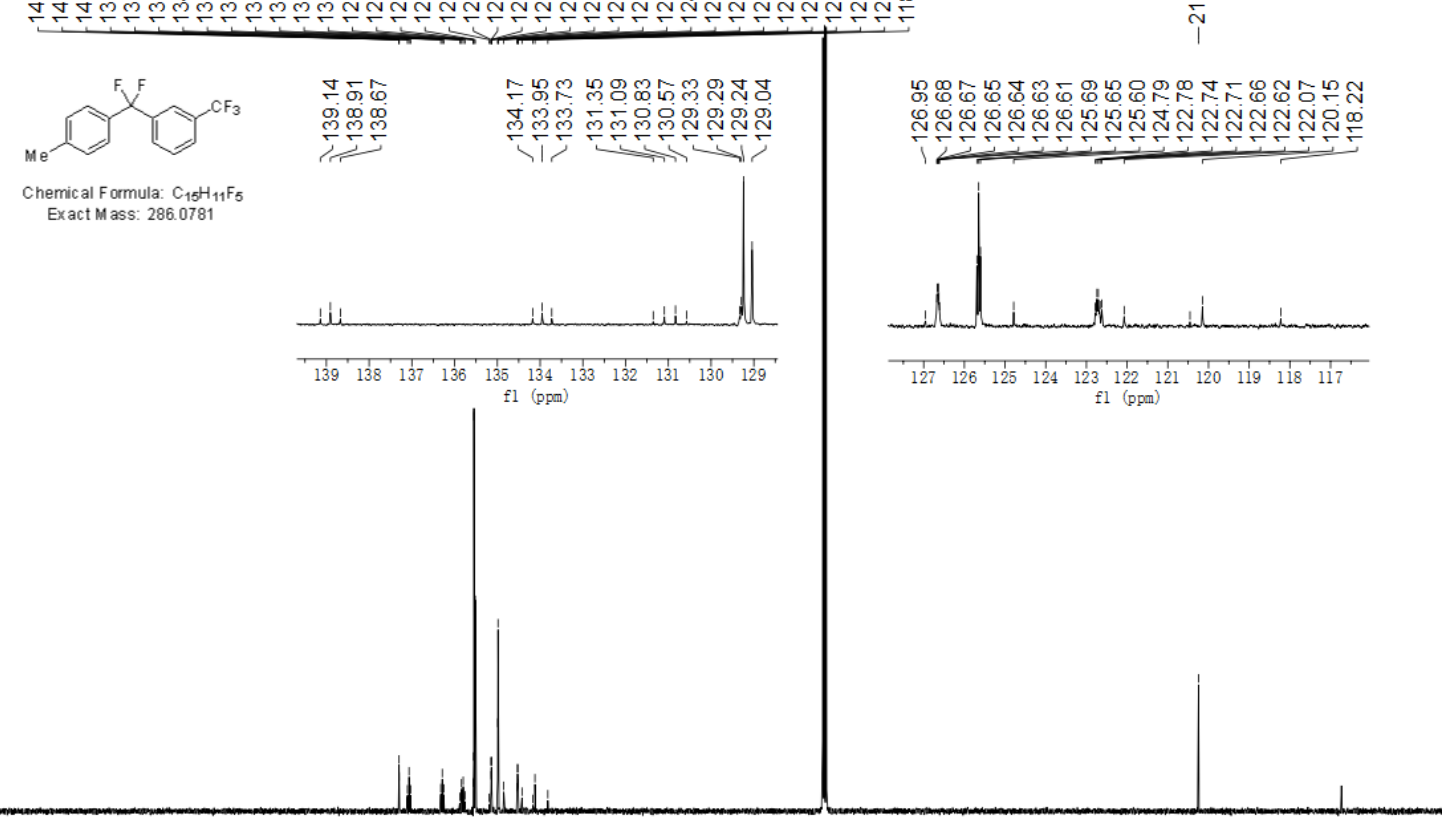

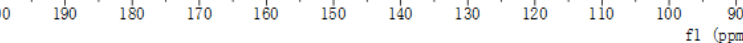


1-((4-(tert-Butyl)phenyl)difluoromethyl)-3-(trifluoromethyl)benzene: (3d)

${ }^{1} \mathrm{H}$ NMR of $\mathbf{3 d}$

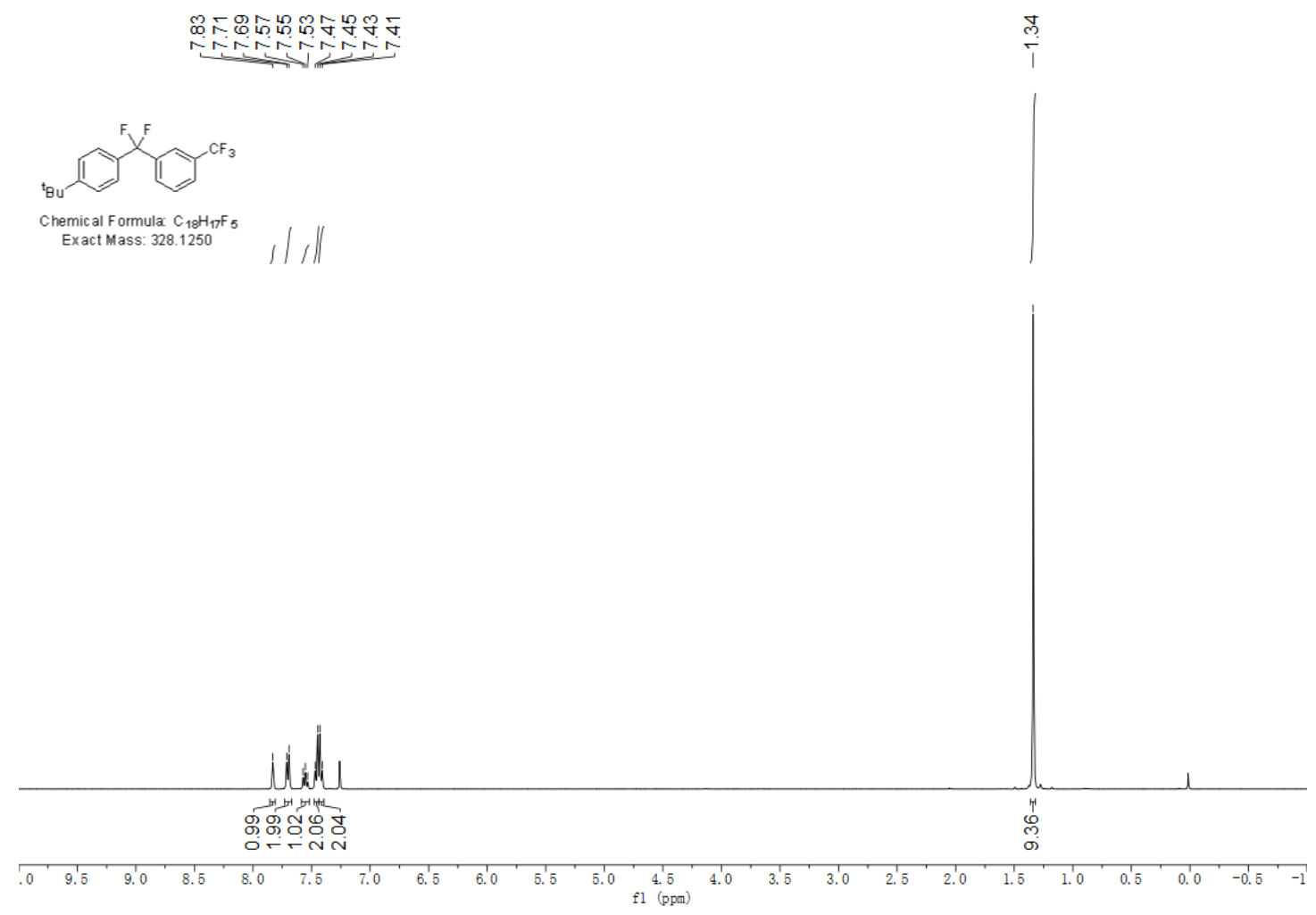

${ }^{19} \mathrm{~F}$ NMR of $\mathbf{3 d}$

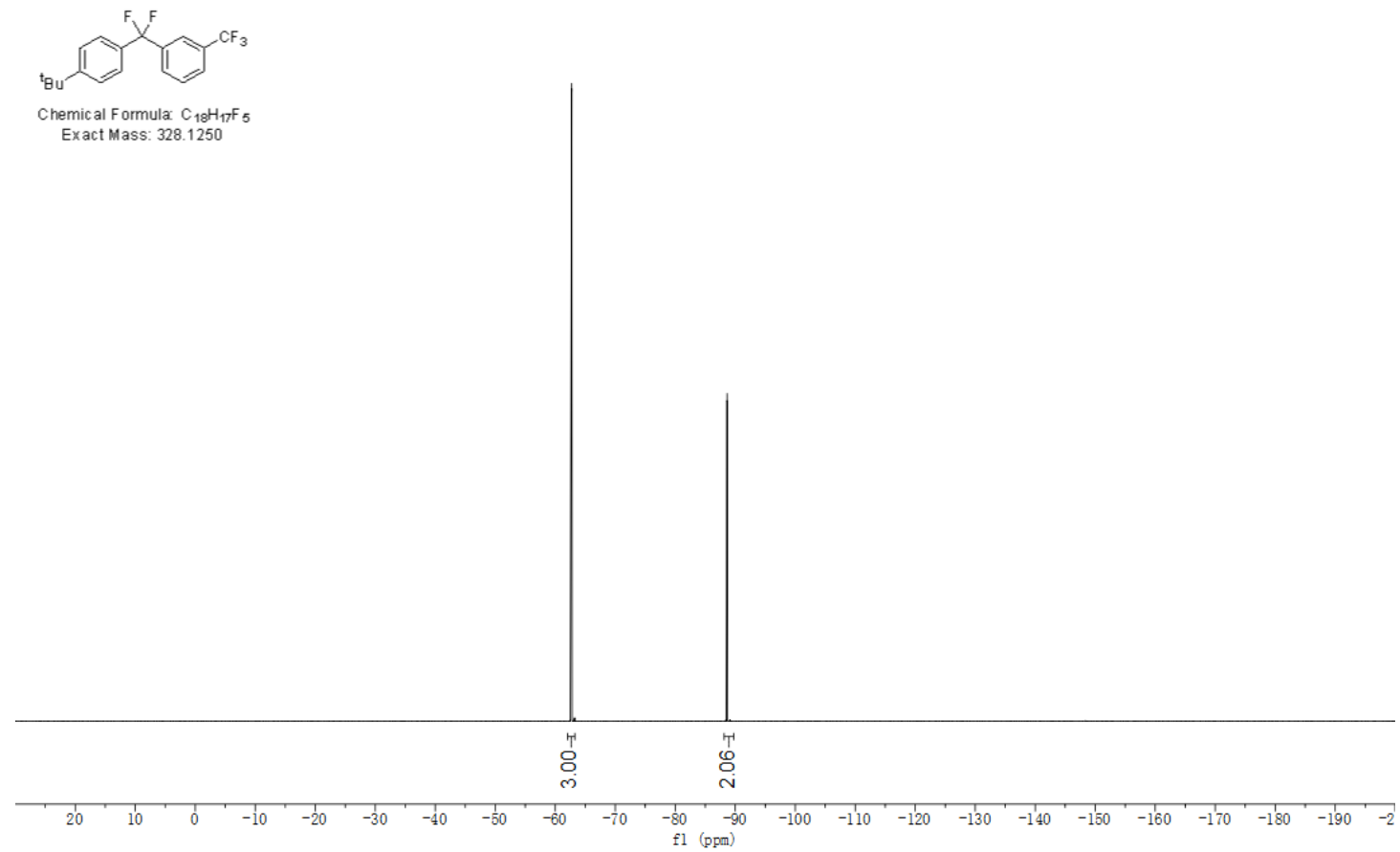


${ }^{13} \mathrm{C}$ NMR of $\mathbf{3 d}$

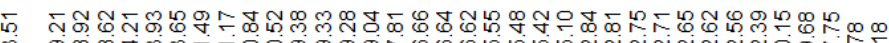

吕
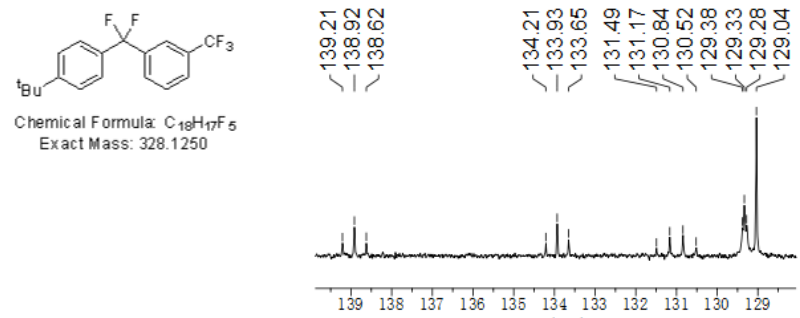

$\begin{array}{llllllllllllllll}139 & 138 & 137 & 136 & 135 & 134 & 133 & 132 & 131 & 130 & 129\end{array}$

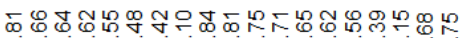

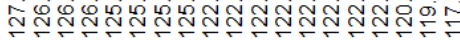

Exact Mass: 328.1250

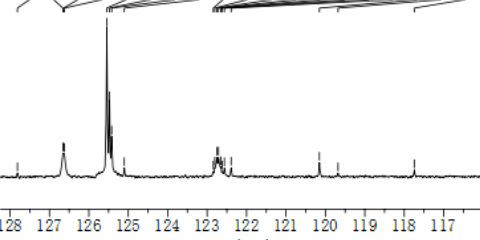

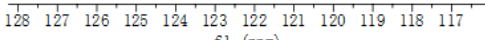
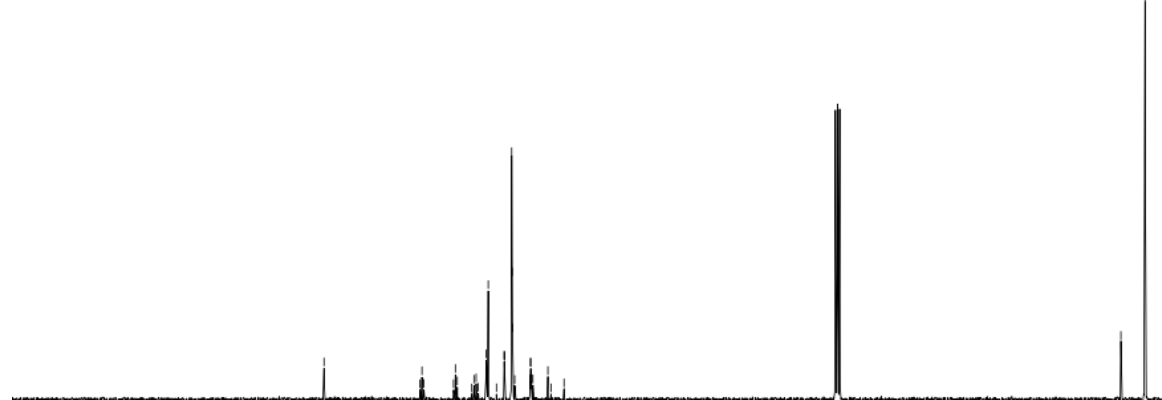

$\begin{array}{lllllllllll}190 & 180 & 170 & 160 & 150 & 140 & 130 & 120 & 110 & 100 & 90\end{array}$

\section{1-((4-Butylphenyl)difluoromethyl)-3-(trifluoromethyl)benzene (3e)}

${ }^{1} \mathrm{H}$ NMR of $3 \mathbf{e}$

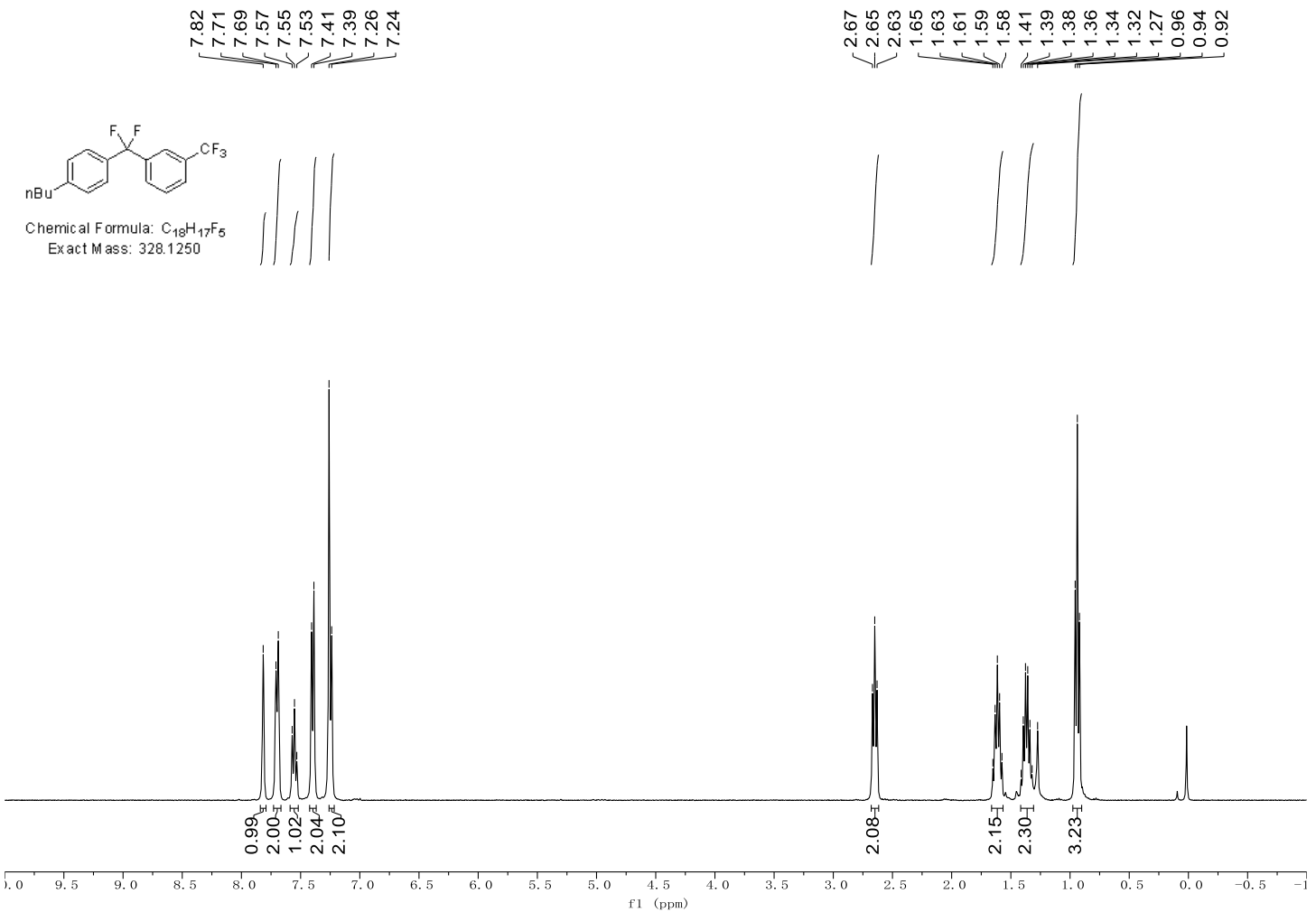


${ }^{19} \mathrm{~F}$ NMR of $\mathbf{3 e}$

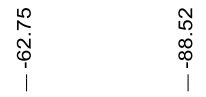

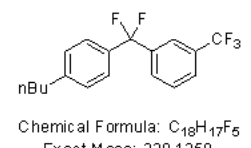

Exact $1025: 3281250$

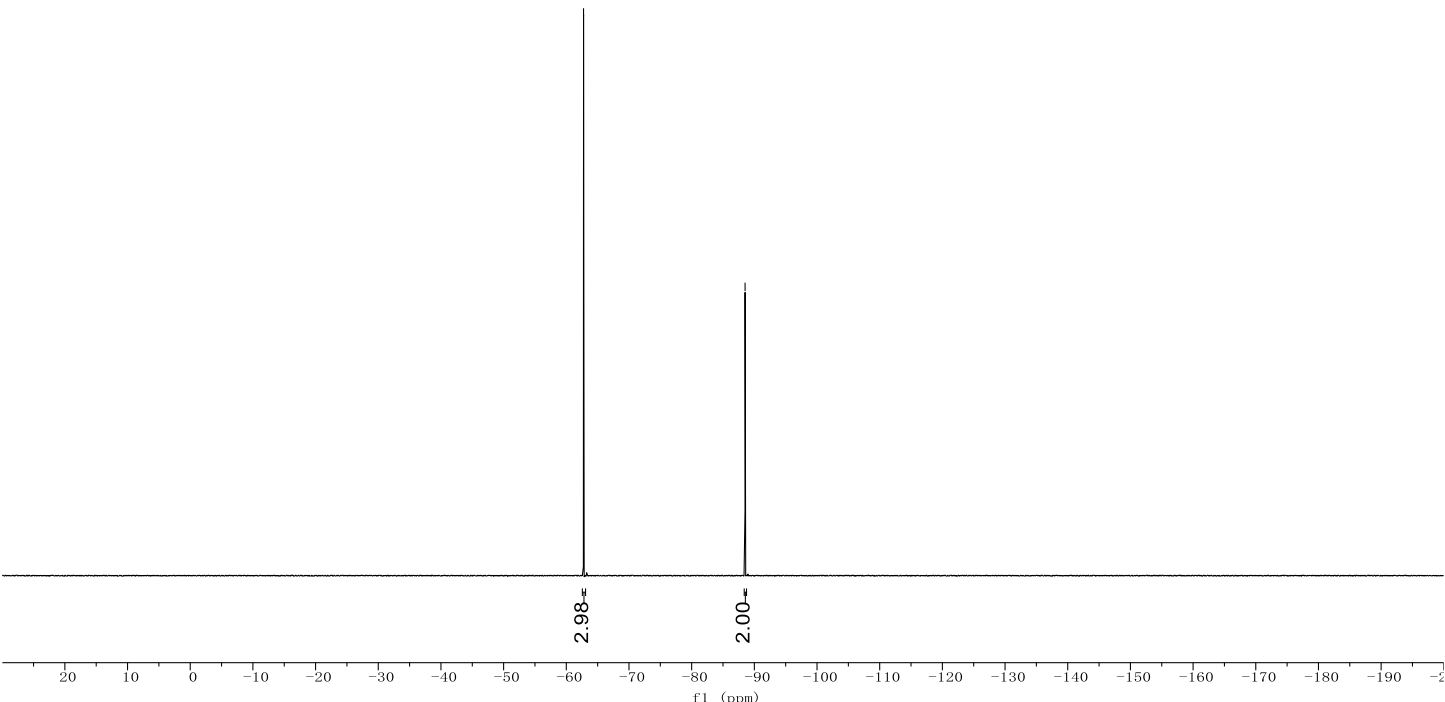

\section{${ }^{13} \mathrm{C}$ NMR of $3 \mathbf{e}$}

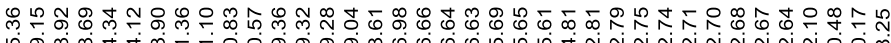

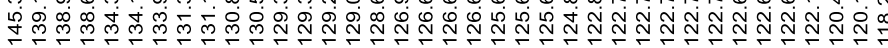

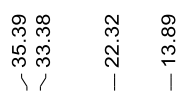

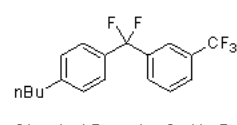

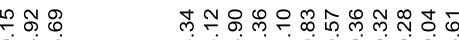

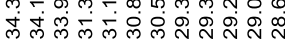

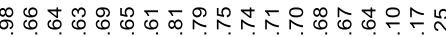

Chemic al Formula: $\mathrm{C}_{18} \mathrm{H}_{17} \mathrm{~F}$

Exact M ass: 328.1250

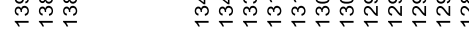

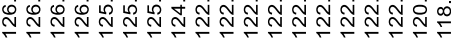

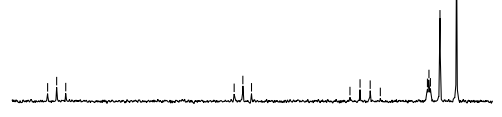

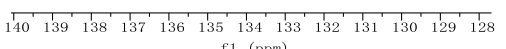

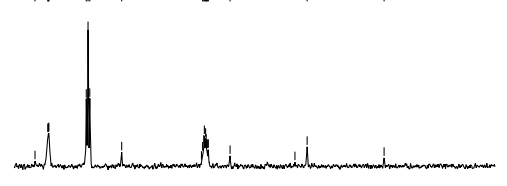
$1(\mathrm{ppm})$
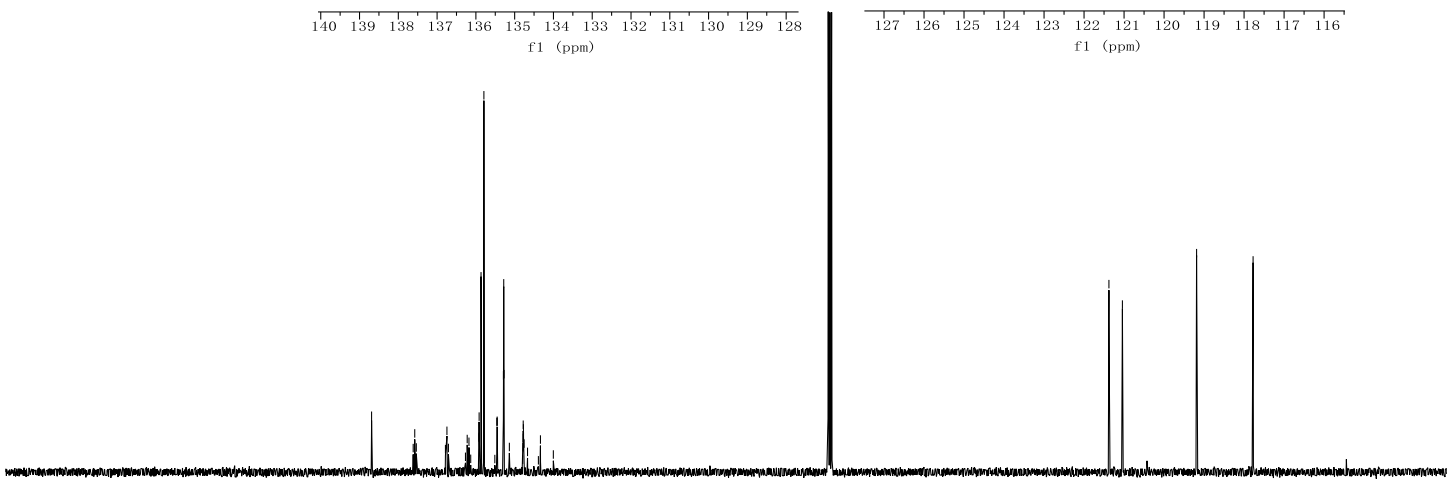

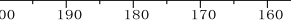

$120+110$

f1 $\quad \begin{gathered}90 \\ (\mathrm{ppm})\end{gathered}$ 
1-(Difluoro(4-phenoxyphenyl)methyl)-3-(trifluoromethyl)benzene (3f)

${ }^{1} \mathrm{H}$ NMR of $\mathbf{3 f}$

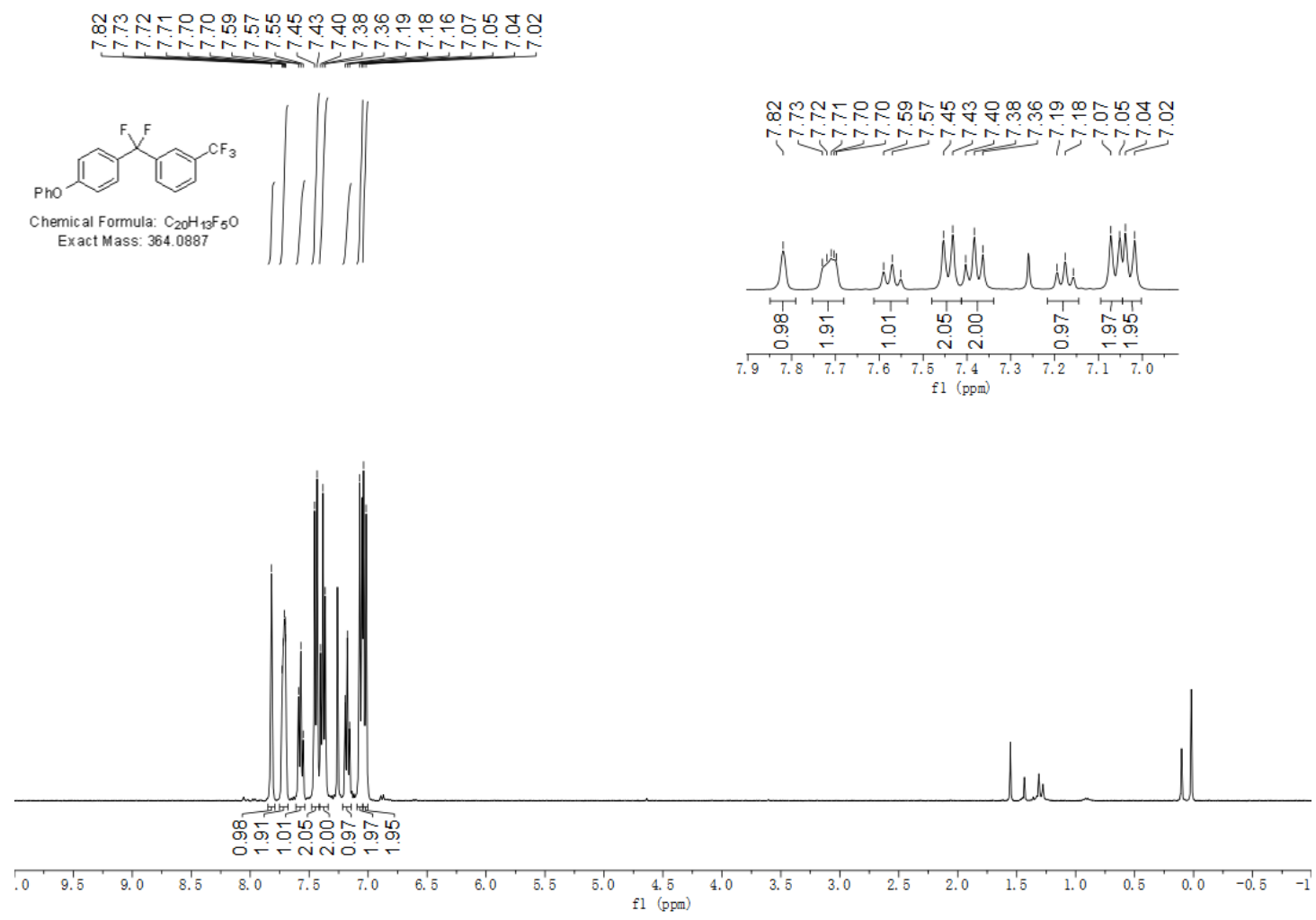

${ }^{19} \mathrm{~F}$ NMR of $\mathbf{3 f}$

$\underset{\substack{0 \\ 0}}{\substack{0 \\ 0}} \stackrel{\substack{0 \\ c}}{N}$

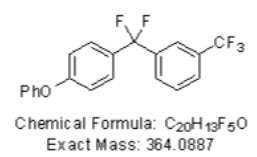

Exact Mass: 364.0887

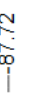

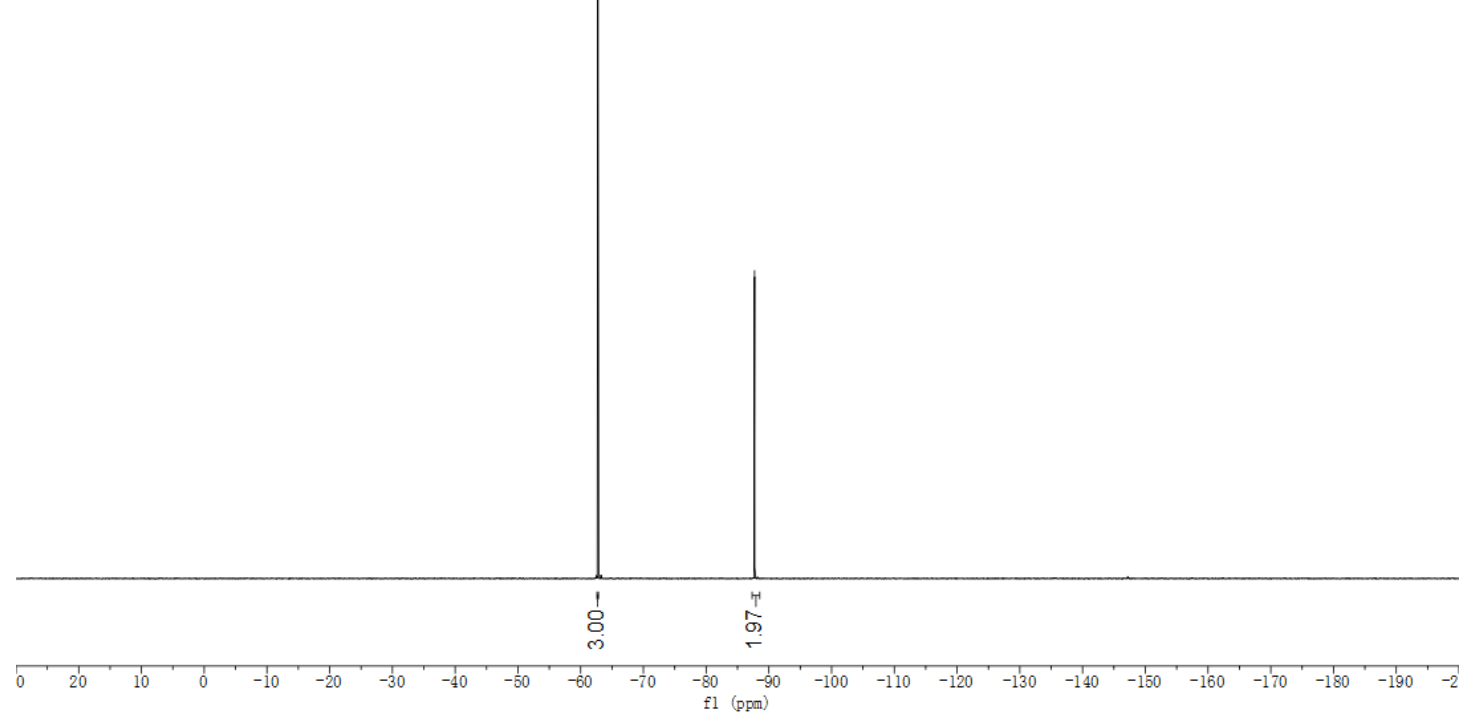


${ }^{13} \mathrm{C}$ NMR of $\mathbf{3 f}$

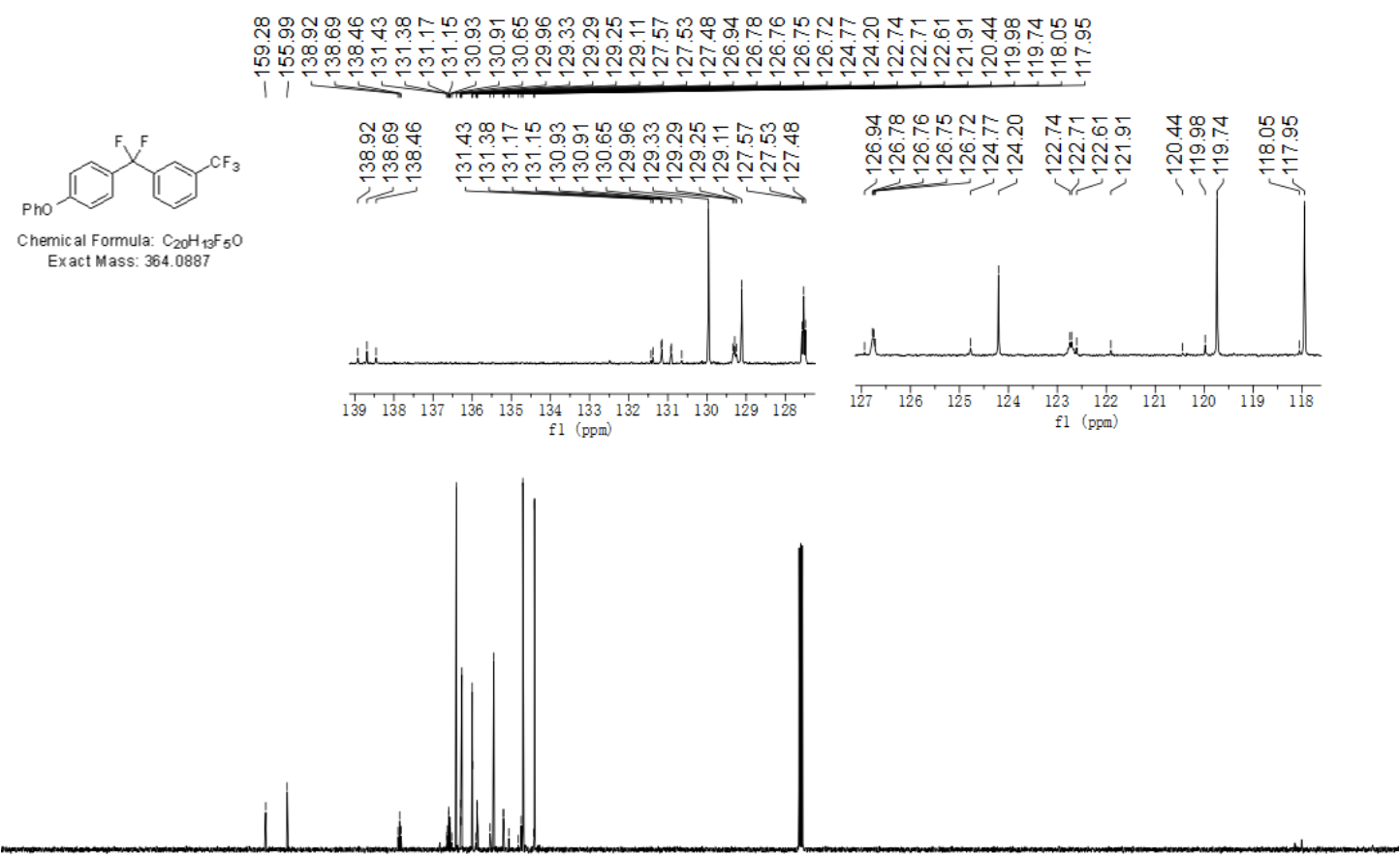

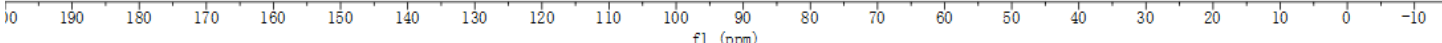

tert-Butyl 5-(difluoro(3-(trifluoromethyl)phenyl)methyl)-1H-indole-1-carboxylate (3g)

${ }^{1} \mathrm{H}$ spectra of $\mathbf{3 g}$

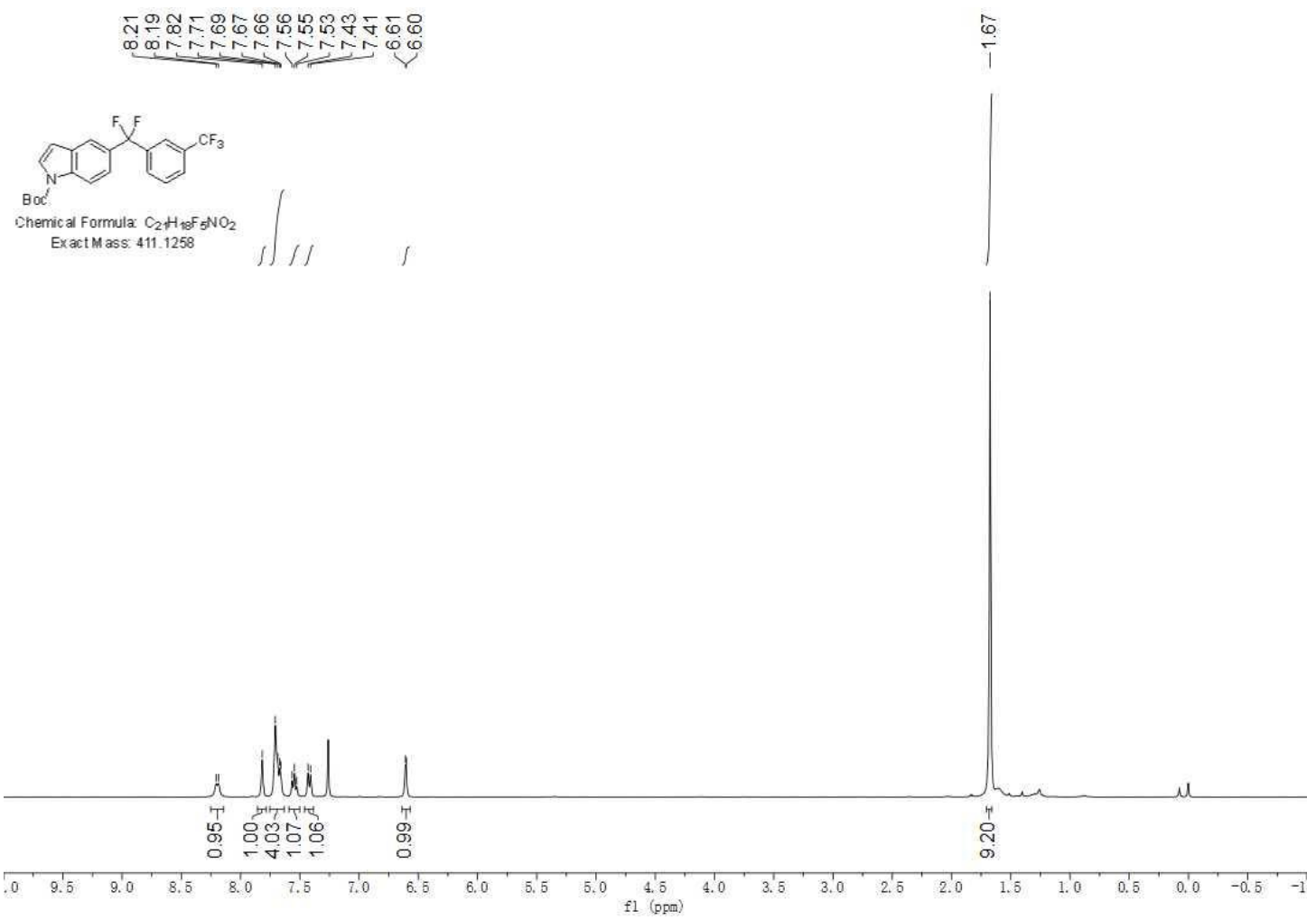


${ }^{19} \mathrm{~F}$ spectra of $\mathbf{3 g}$
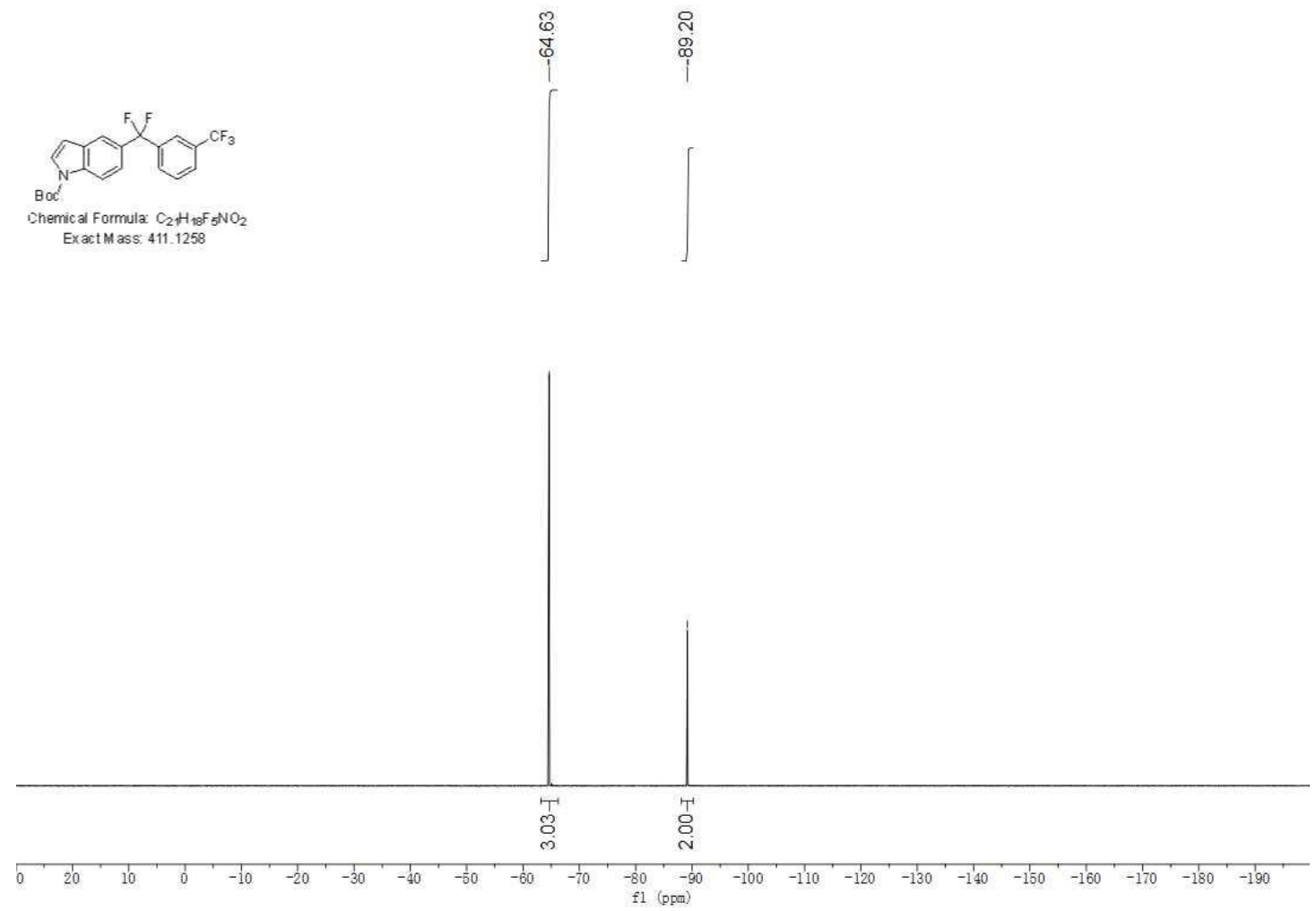

${ }^{13} \mathrm{C}$ spectra of $\mathbf{3 g}$

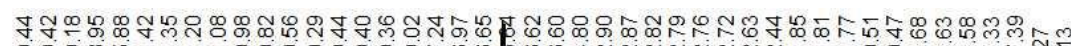

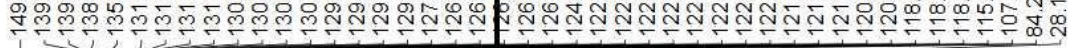

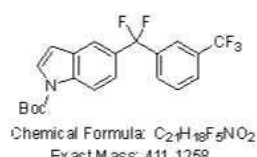

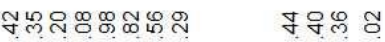

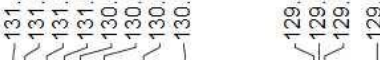
Exact Mass: 411.1258 iั
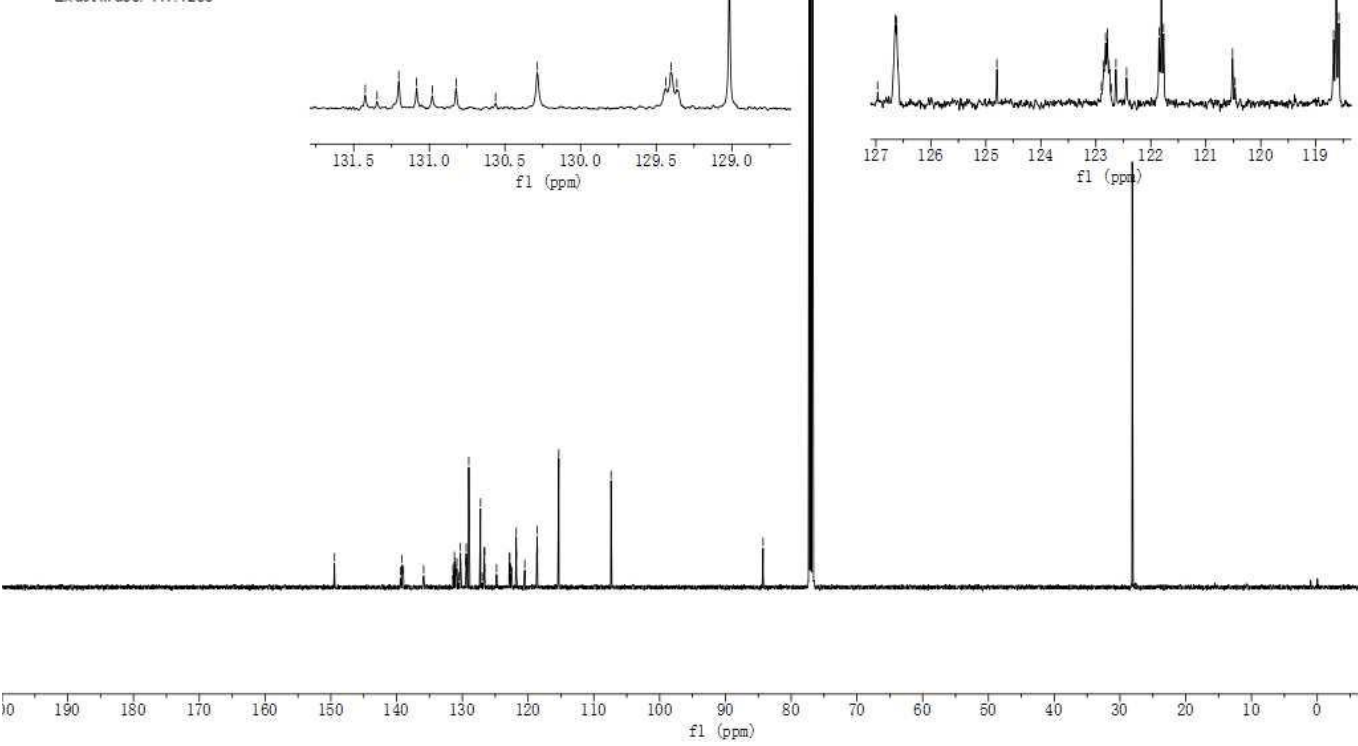
(4-(Difluoro(3-(trifluoromethyl)phenyl)methyl)phenyl)(methyl)sulfane (3h)

${ }^{1} \mathrm{H}$ NMR of $\mathbf{3 h}$
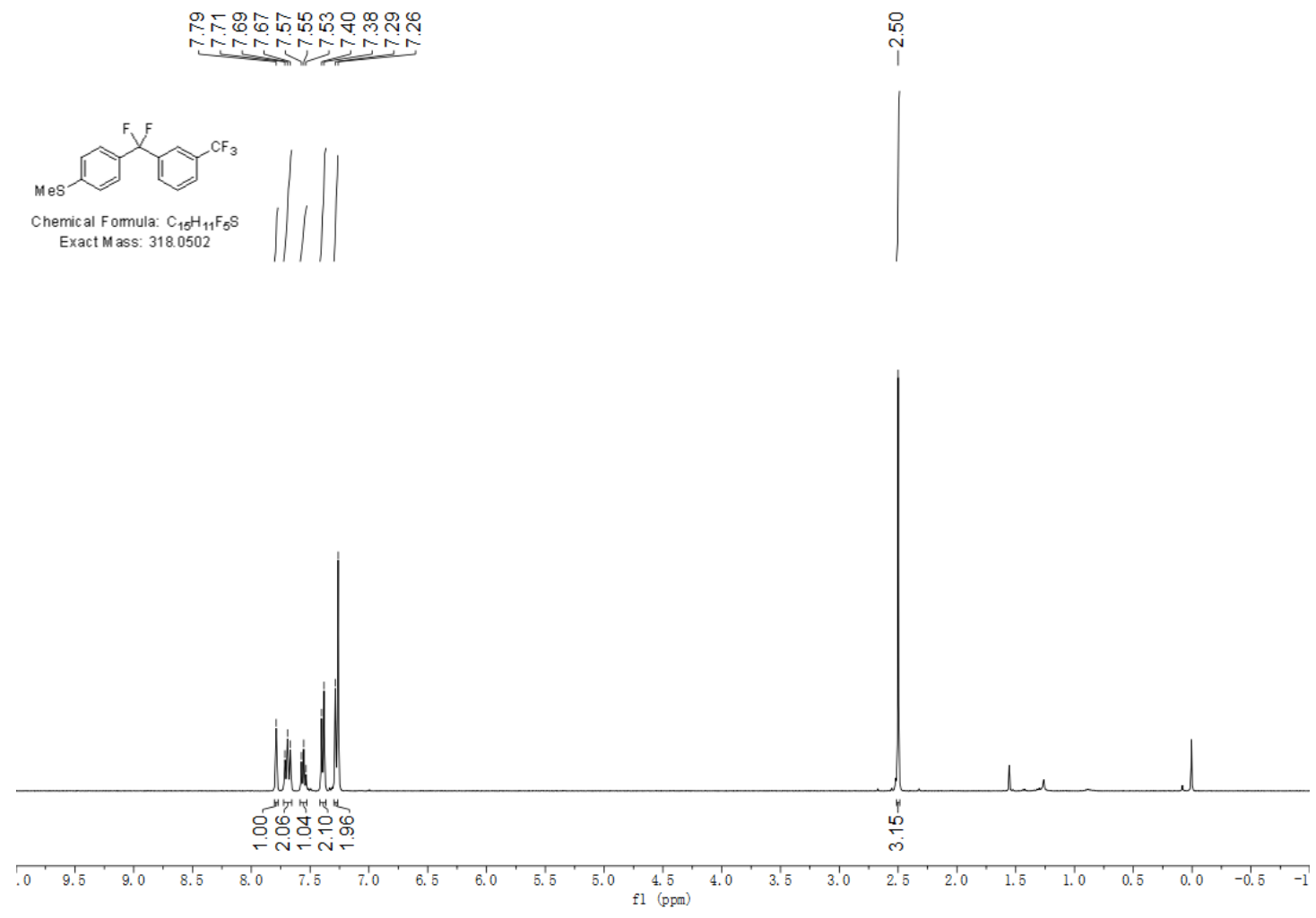

${ }^{19}$ F NMR of $\mathbf{3 h}$

$\begin{array}{ll}\infty & N \\ & \infty \\ 0 & \infty \\ 1 & 1\end{array}$
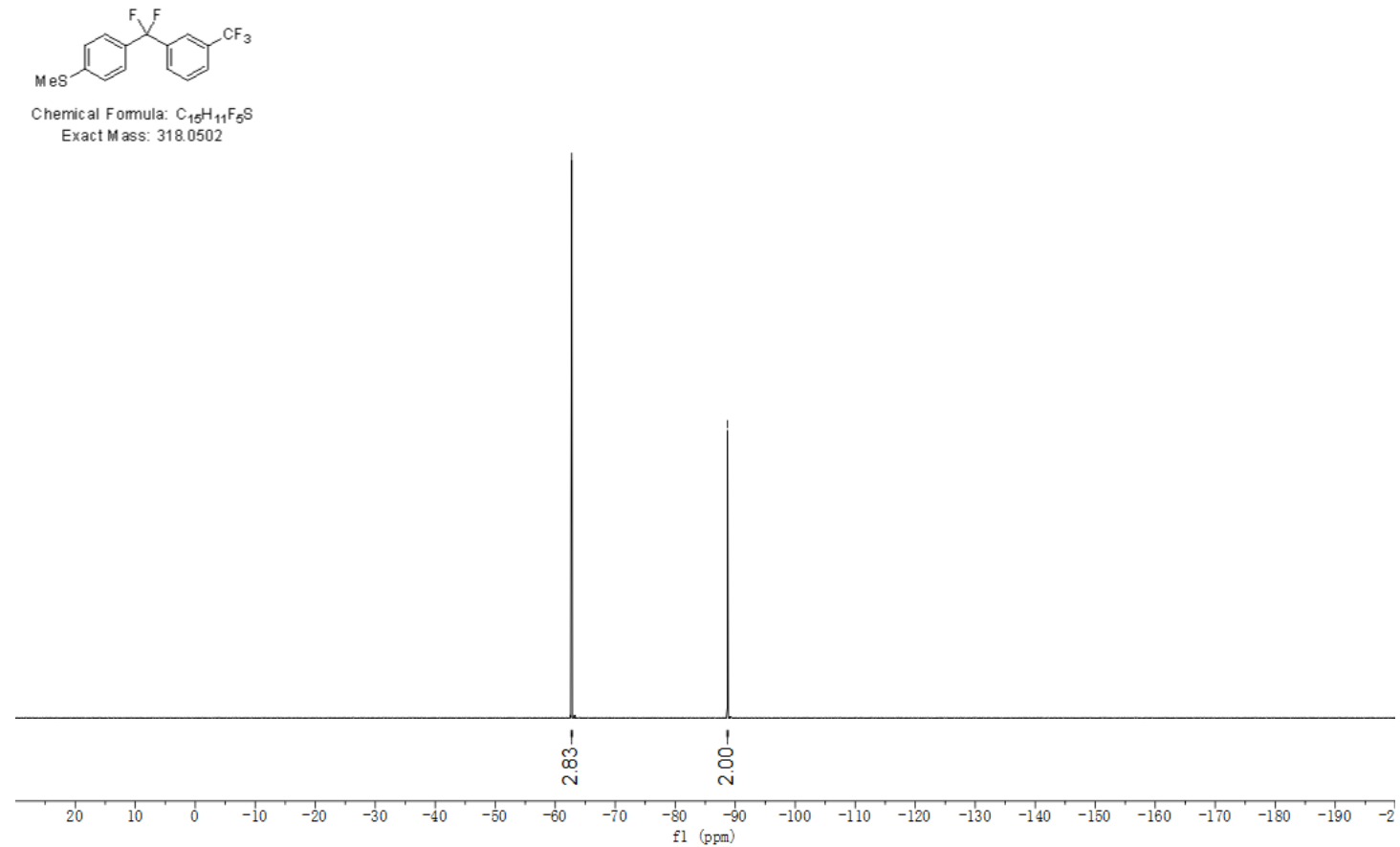


\section{${ }^{13} \mathrm{C}$ NMR of $3 \mathbf{h}$}

๓

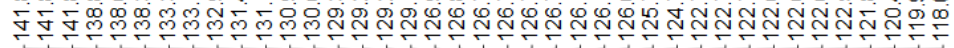

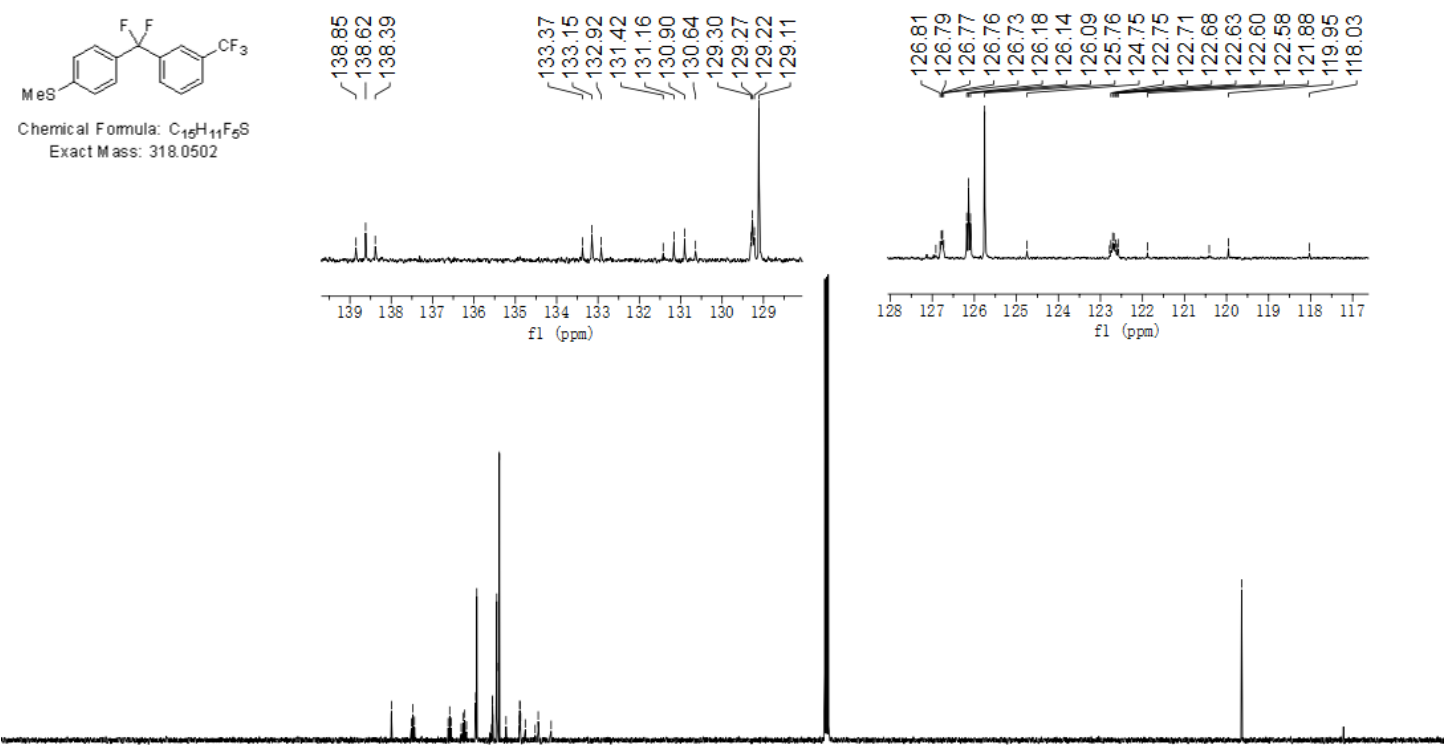

$\begin{array}{lllllllllll}190 & 180 & 170 & 160 & 150 & 140 & 130 & 120 & 110 & 100 & 90\end{array}$

\section{1-(Difluoro(3-(trifluoromethyl)phenyl)methyl)-3-methoxybenzene (3i)}

${ }^{1} \mathrm{H}$ NMR of $3 \mathbf{i}$

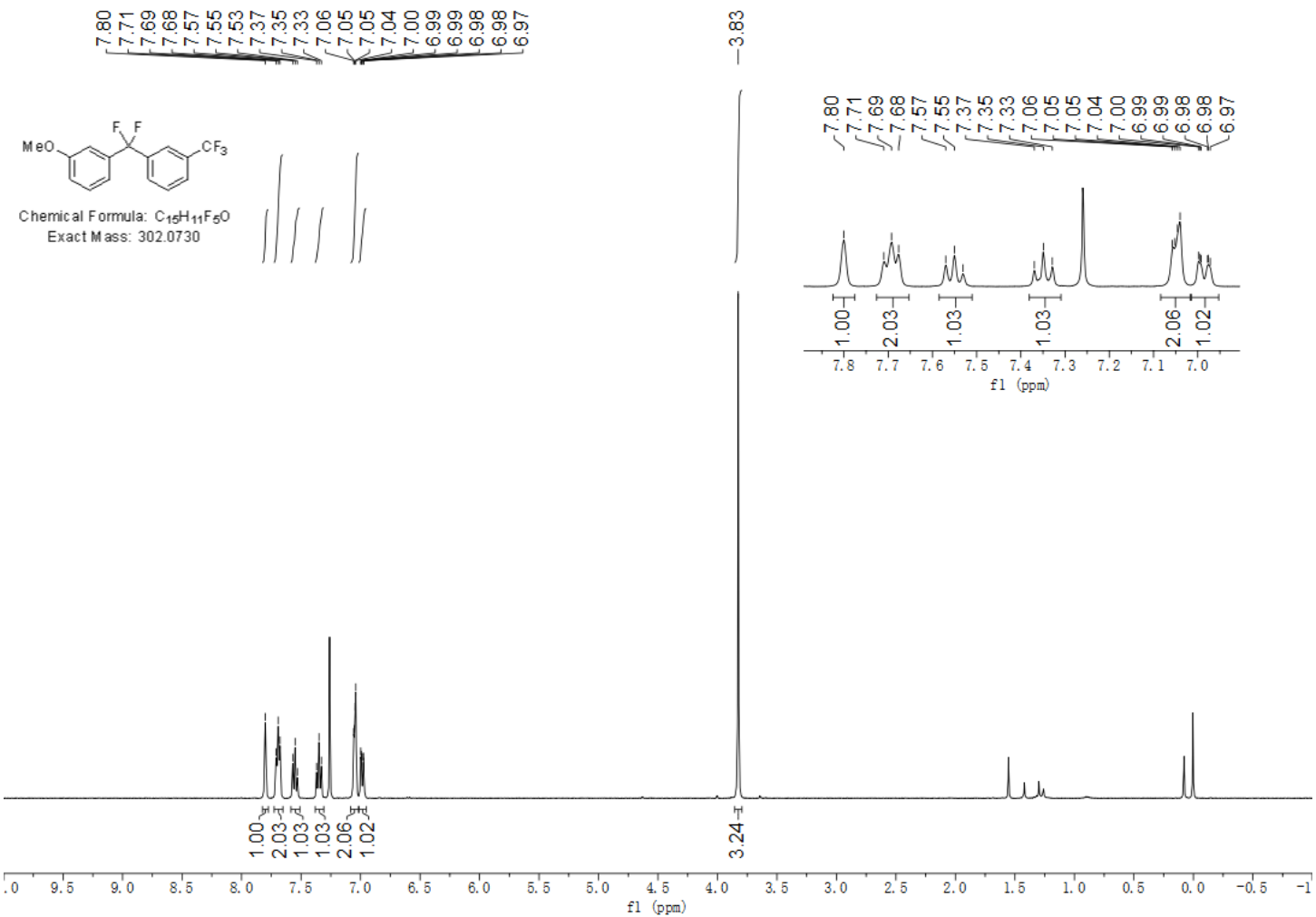


${ }^{19}$ F NMR of 3i

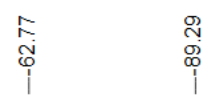

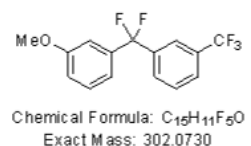

Exact Mass: 302.0730

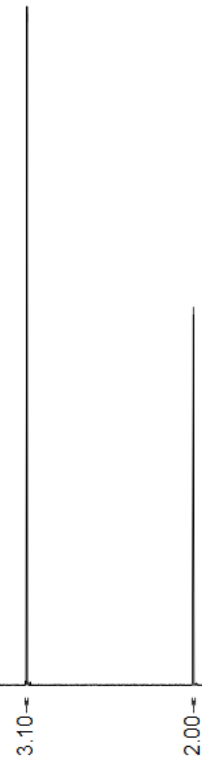

인

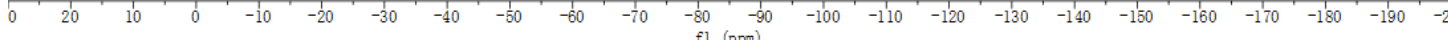

${ }^{13} \mathrm{C}$ NMR of $3 \mathbf{i}$

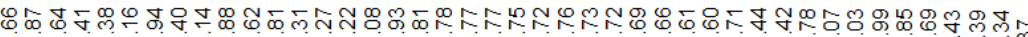

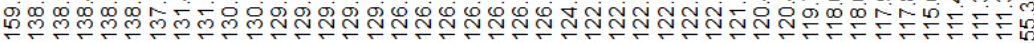

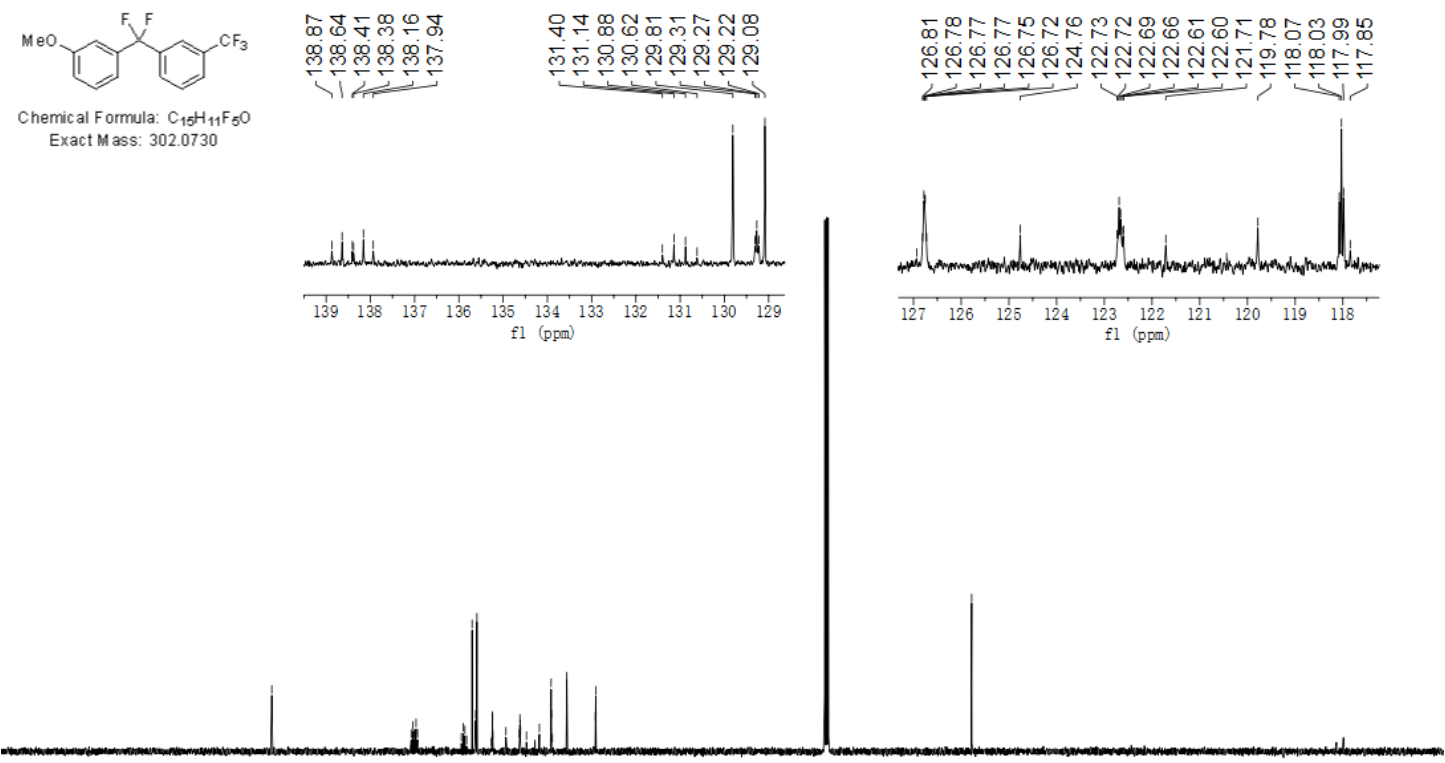

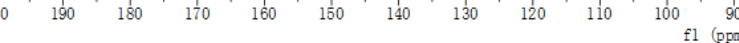


$N$-(3-(Difluoro(3-(trifluoromethyl)phenyl)methyl)phenyl)acetamide (3j)

${ }^{1} \mathrm{H}$ NMR of $\mathbf{3 j}$
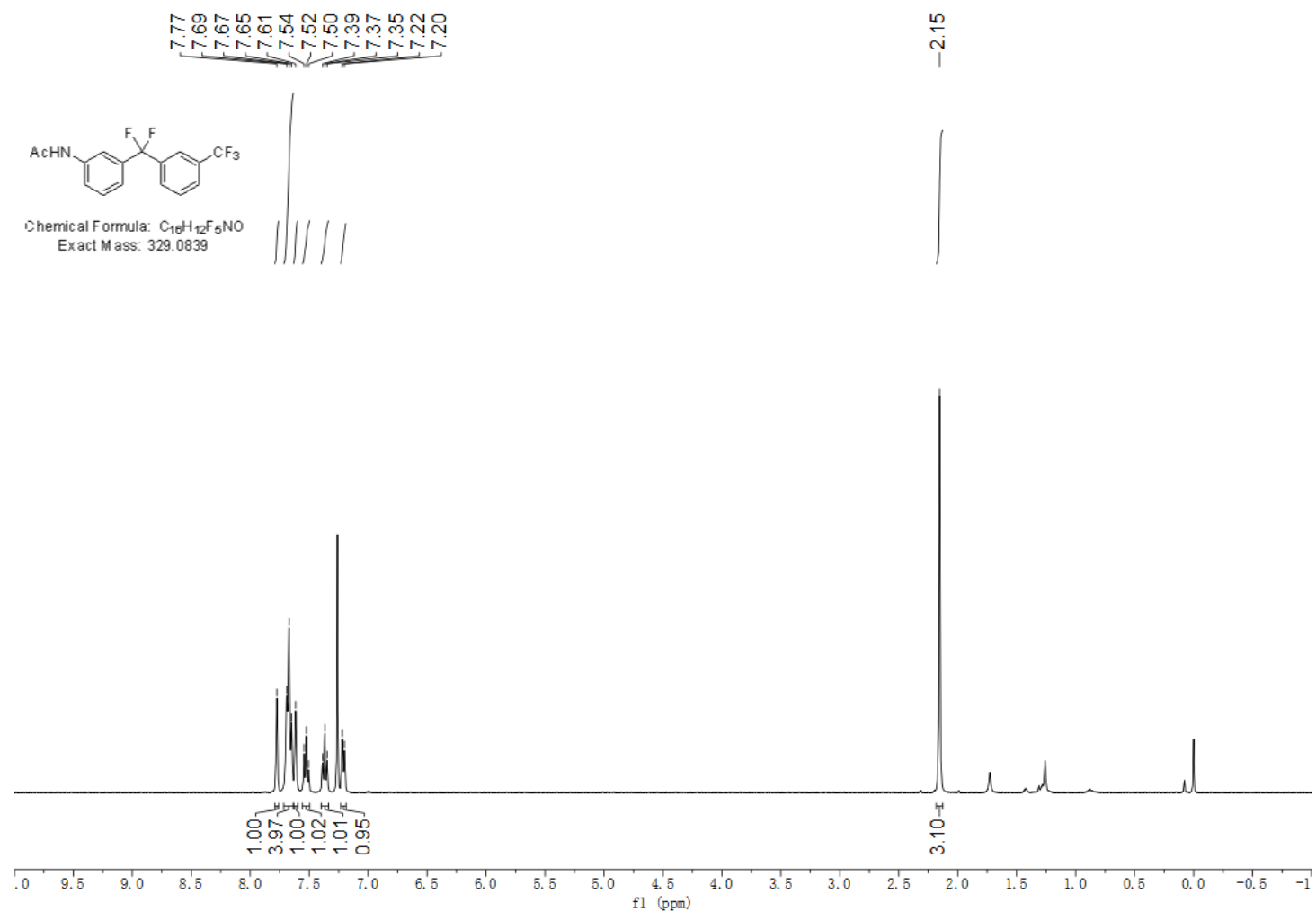

${ }^{19}$ F NMR of $\mathbf{3 j}$

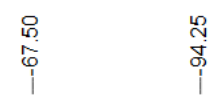
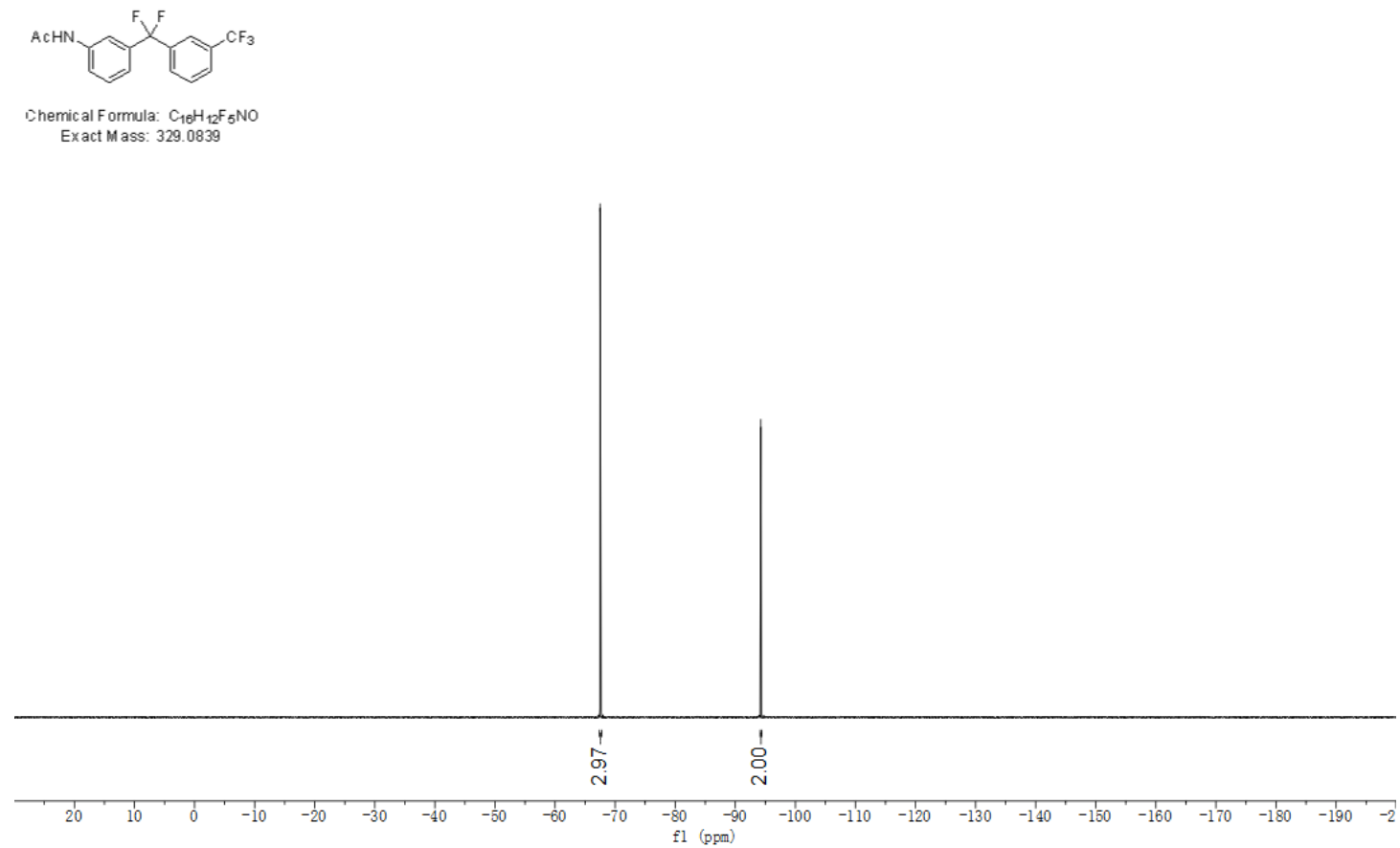
${ }^{13} \mathrm{C}$ NMR of $\mathbf{3 j}$

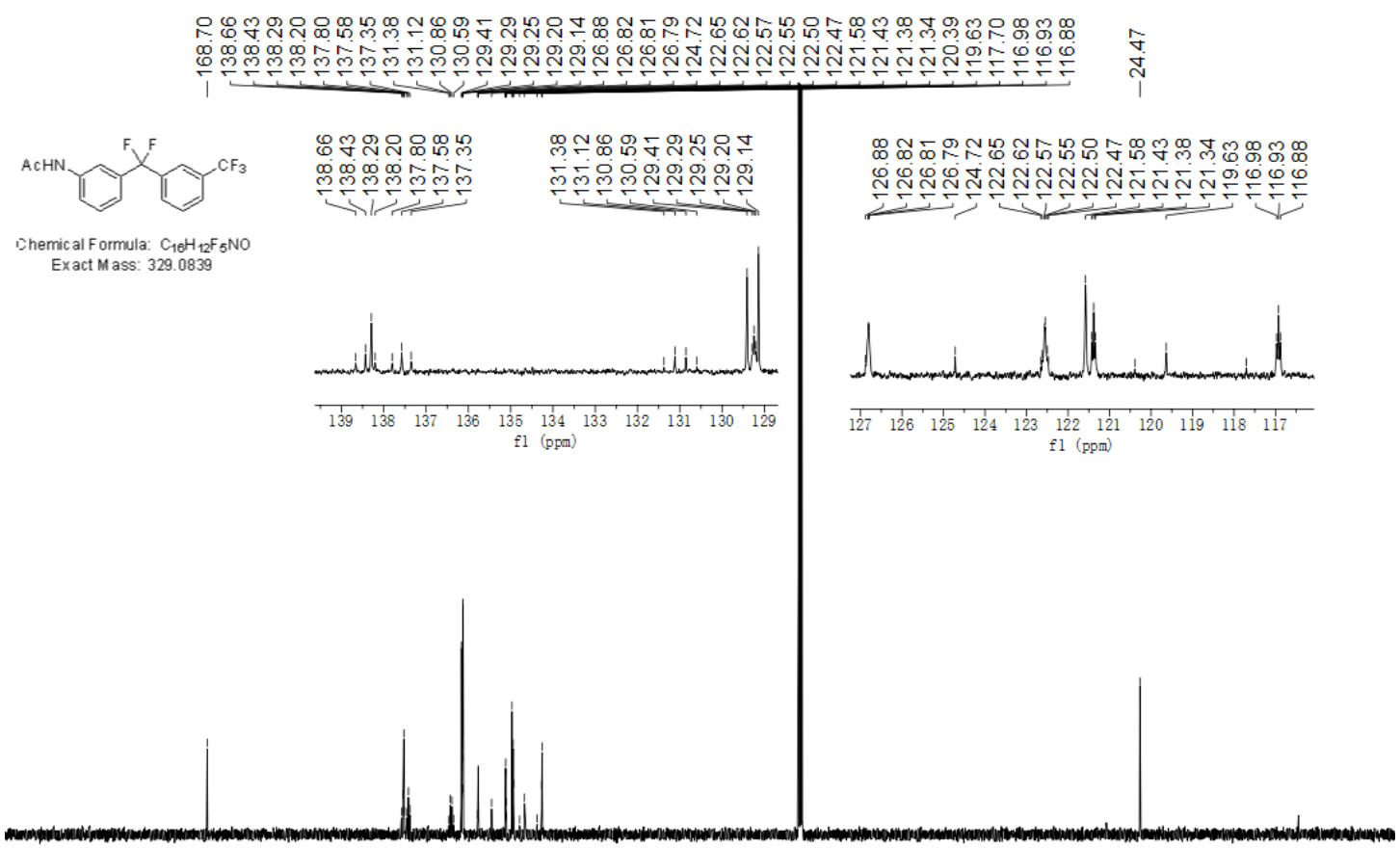

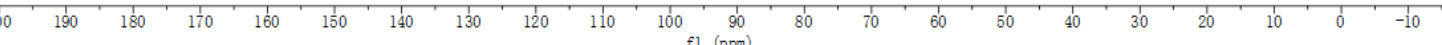

\section{1-(Difluoro(p-tolyl)methyl)-2-(trifluoromethyl)benzene (3k)}

${ }^{1} \mathrm{H}$ NMR of $\mathbf{3 k}$

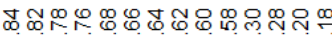

MMNMNRTMNON

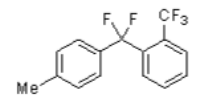

Chemical Formula: $\mathrm{C}_{15} \mathrm{H}_{11} \mathrm{~F}_{5}$ Exact Mass: 286.0781
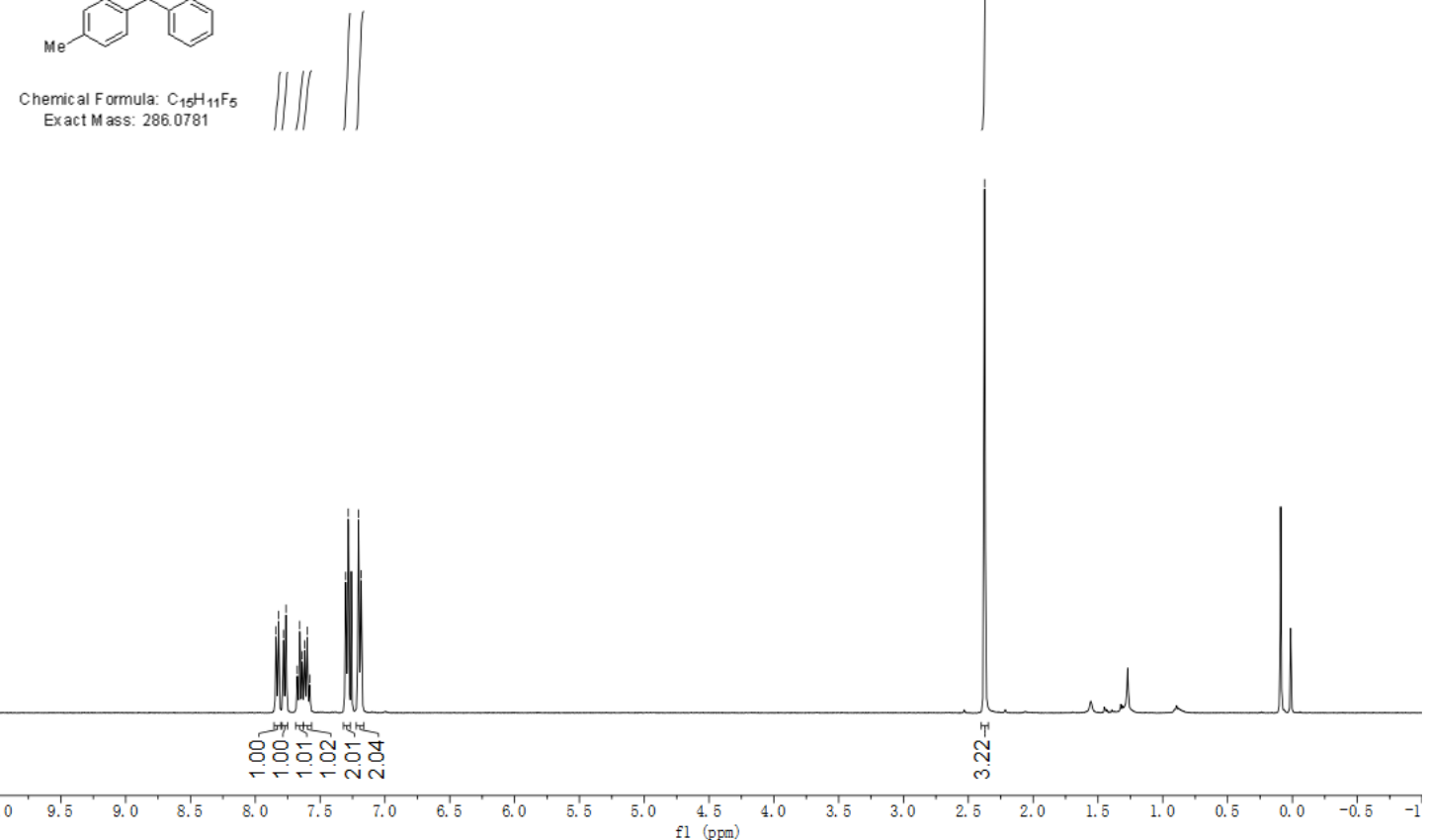
${ }^{19}$ F NMR of $\mathbf{3 k}$

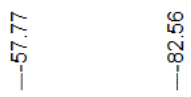

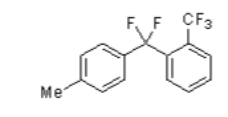

Chemic al Formula: $\mathrm{C}_{15} \mathrm{H}_{11} \mathrm{~F}_{5}$

Exact Mass: 286.0781

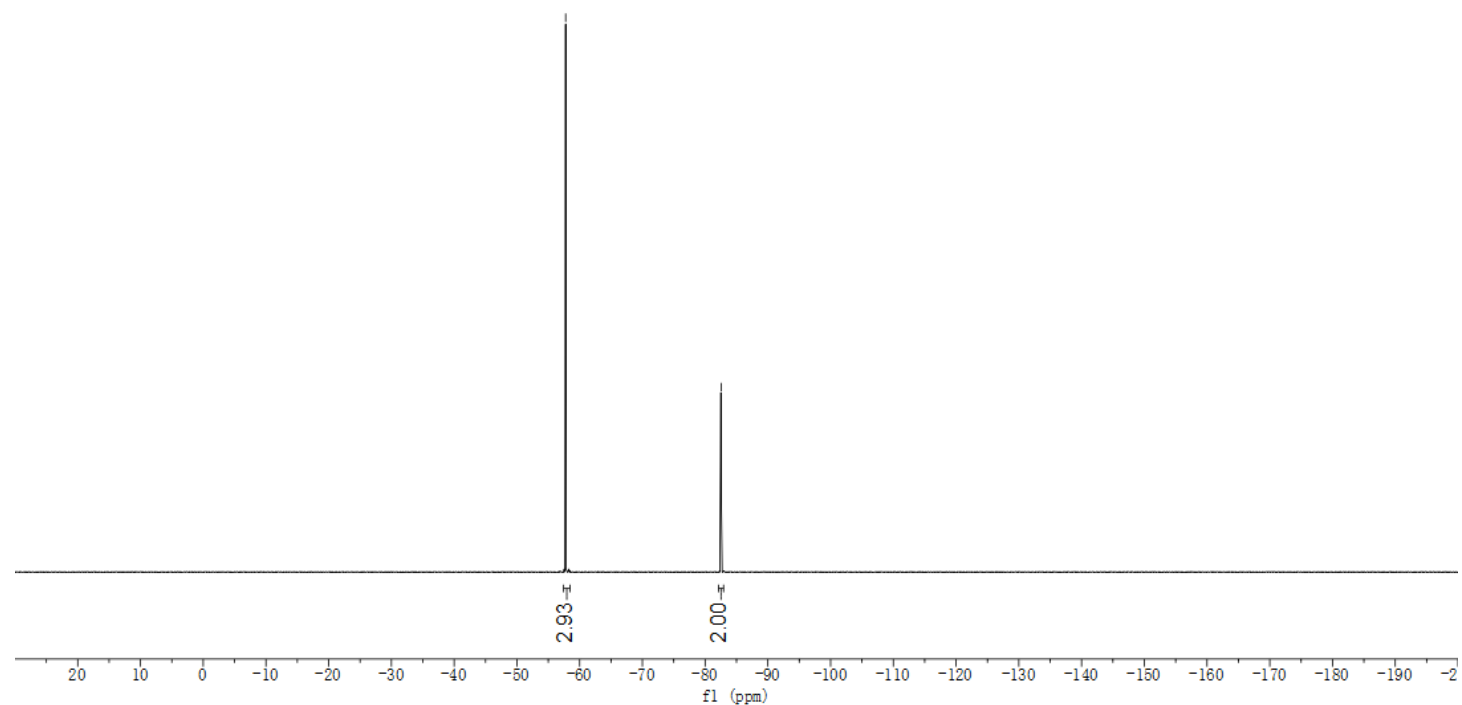

${ }^{13} \mathrm{C}$ NMR of $\mathbf{3 k}$

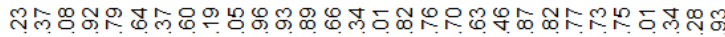

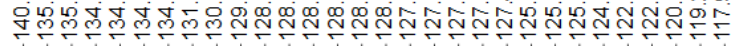

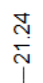

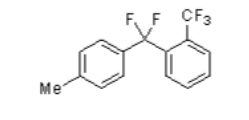

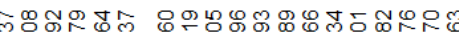

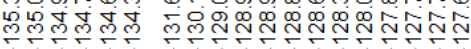

Chemic al Formula: $\mathrm{C}_{15} \mathrm{H}_{11} \mathrm{~F}$ Exact Mass: 286.0781

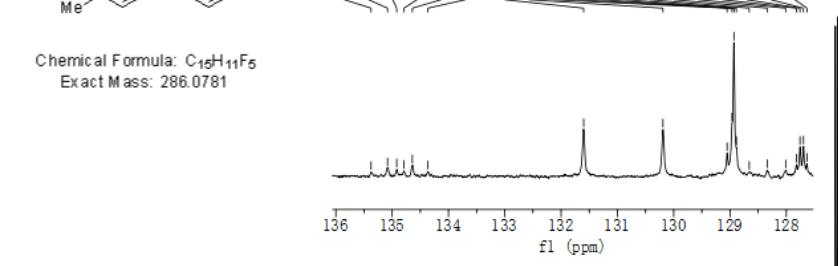

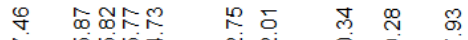

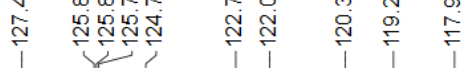
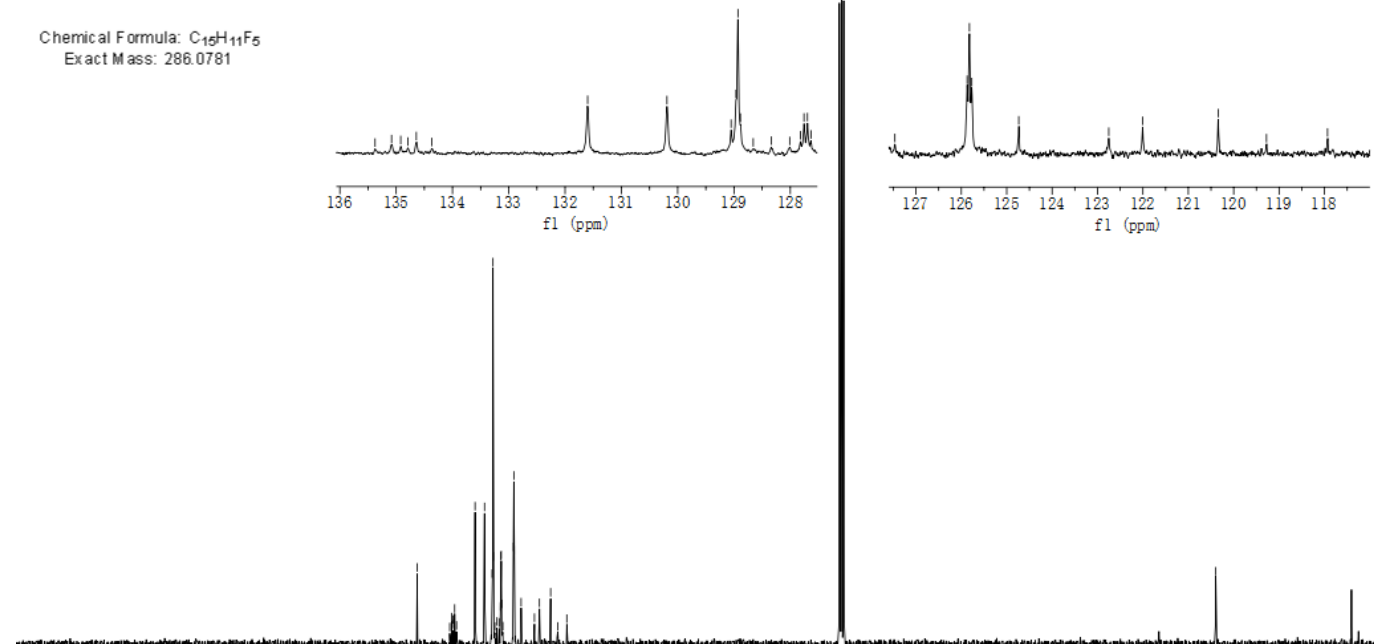

$\begin{array}{llllllllllll}10 & 190 & 180 & 170 & 160 & 150 & 140 & 130 & 120 & 110 & 100 & 90\end{array}$ 
1-(Difluoro(p-tolyl)methyl)-3-methoxy-5-(trifluoromethyl)benzene (3I)

${ }^{1} \mathrm{H}$ NMR of 31
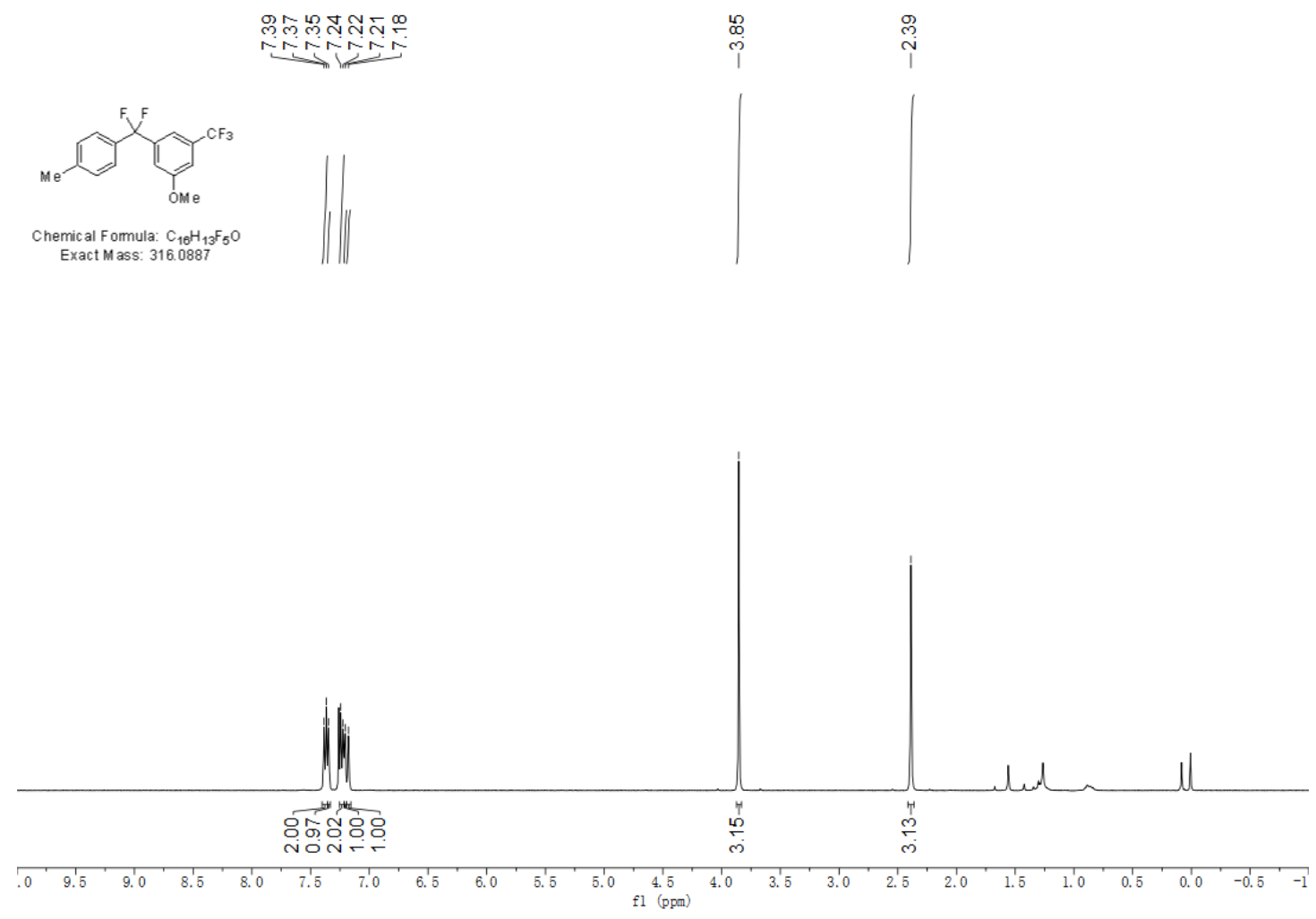

${ }^{19} \mathrm{~F}$ NMR of 31

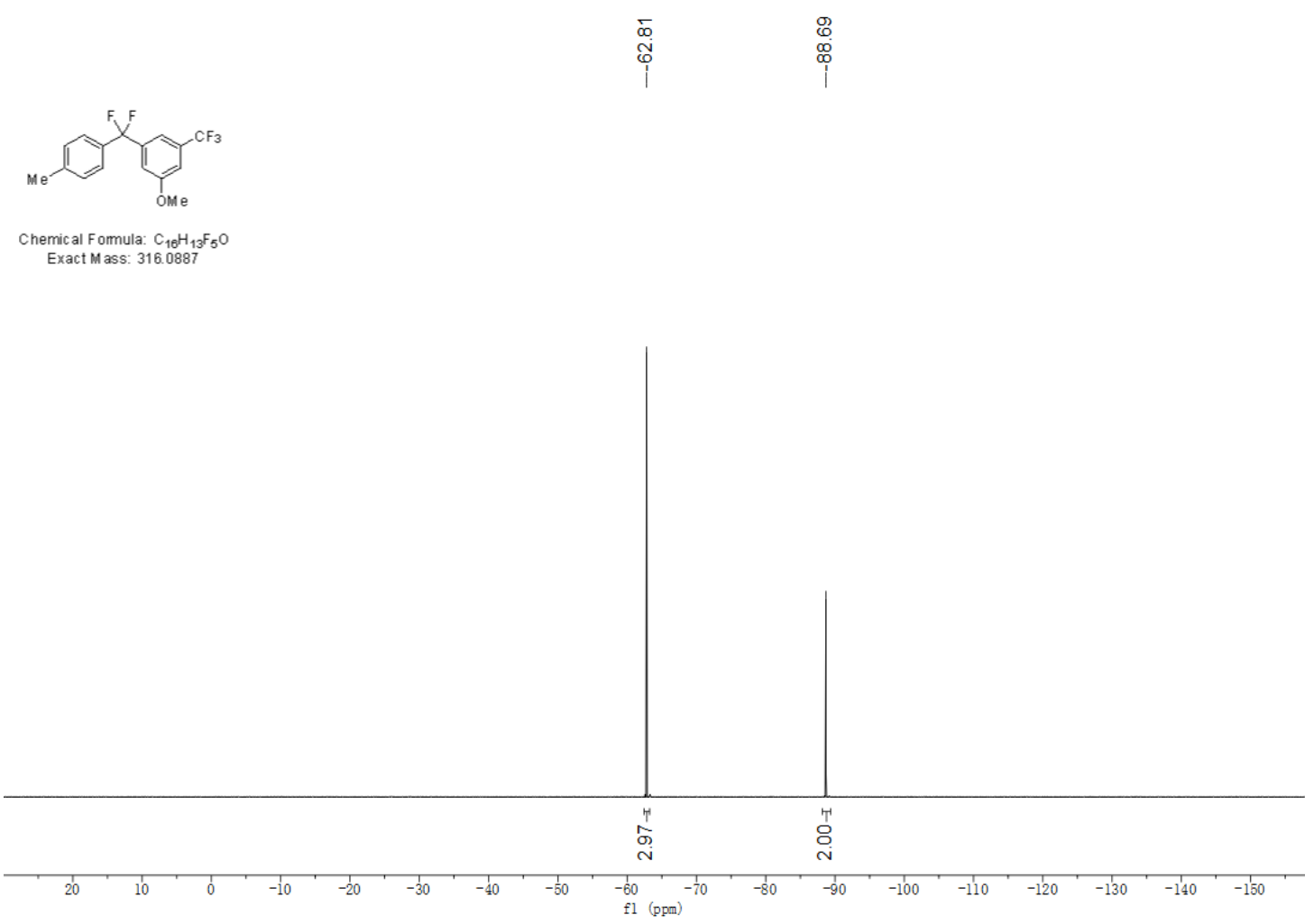


${ }^{13} \mathrm{C}$ NMR of $3 \mathbf{l}$

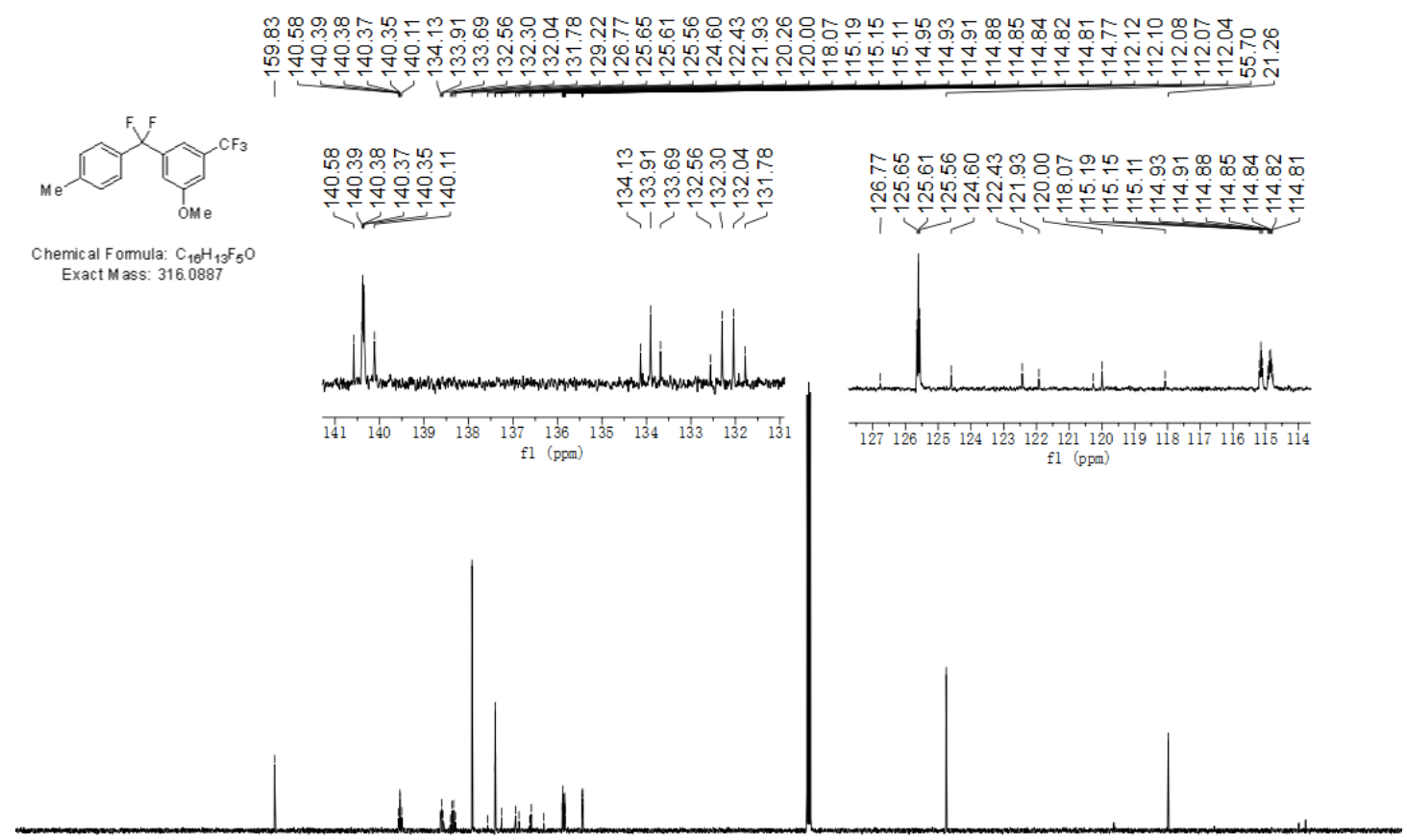

1-(Difluoro(p-tolyl)methyl)-3-(methoxymethyl)-5-(trifluoromethyl)benzene (3m)

${ }^{1} \mathrm{H}$ NMR of $\mathbf{3 m}$
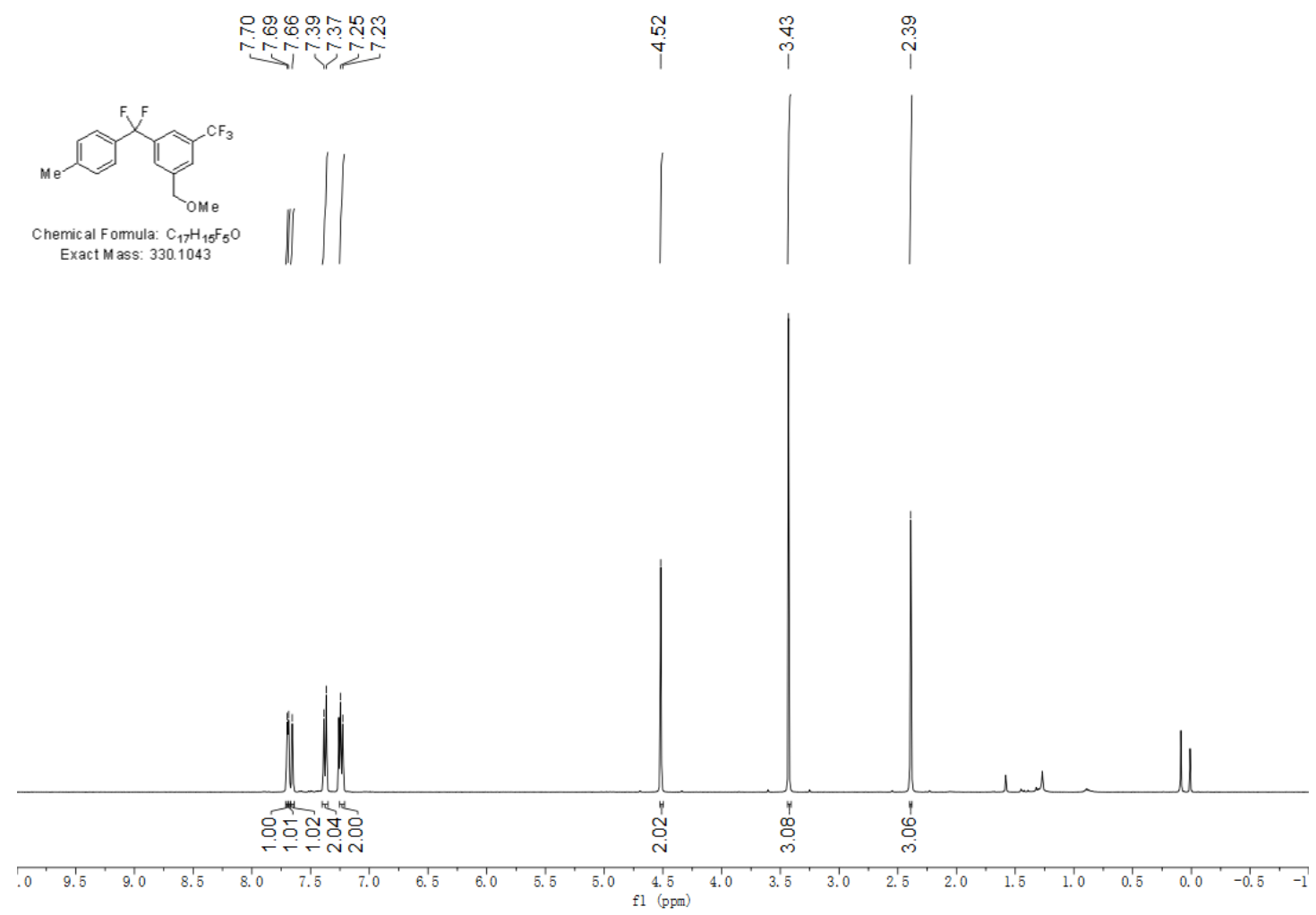
${ }^{19} \mathrm{~F}$ NMR of $\mathbf{3 m}$

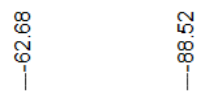
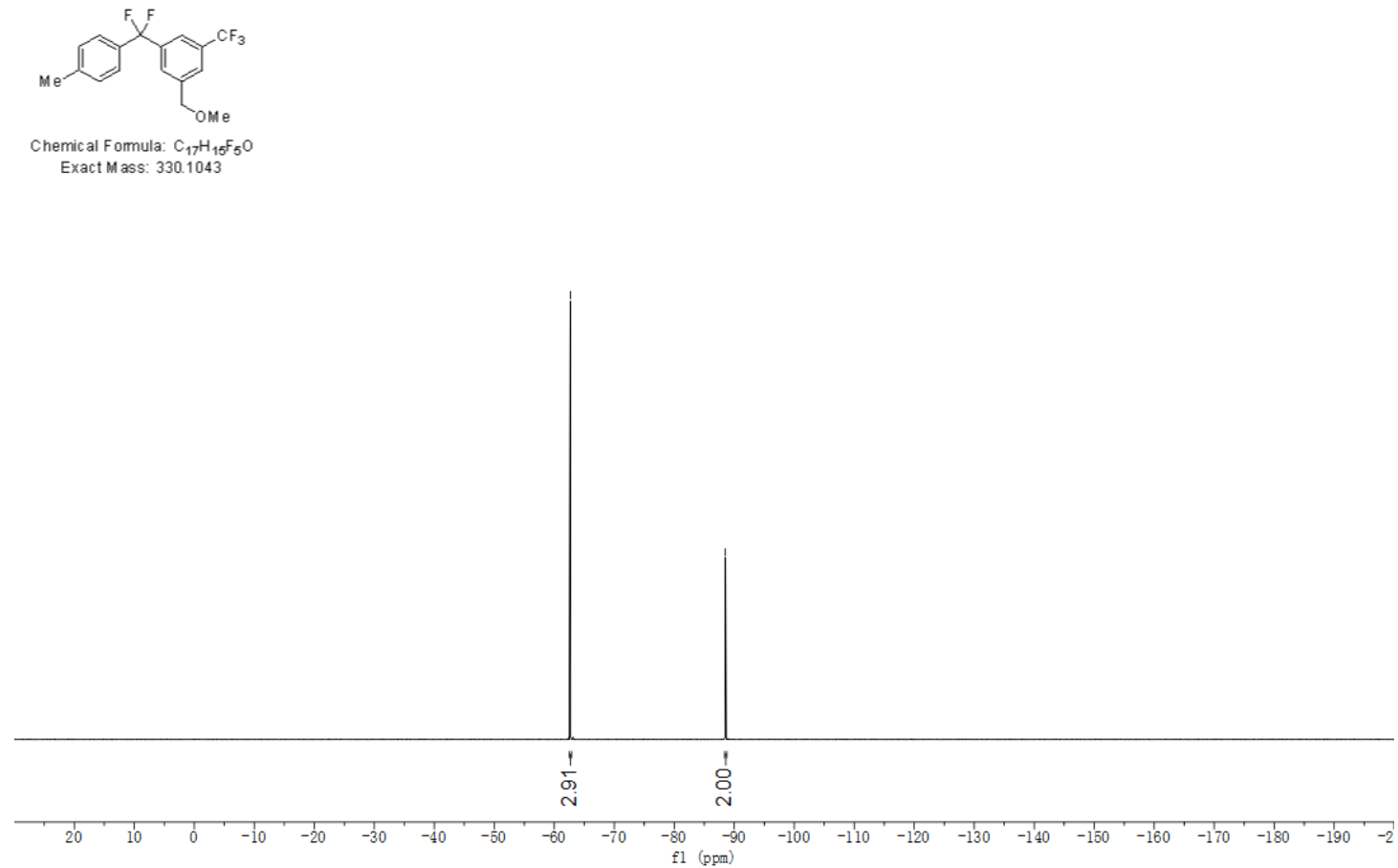

${ }^{13} \mathrm{C}$ NMR of $\mathbf{3 m}$

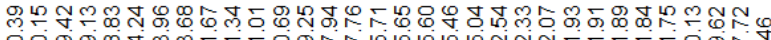

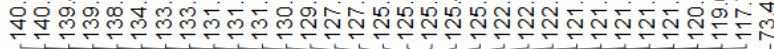
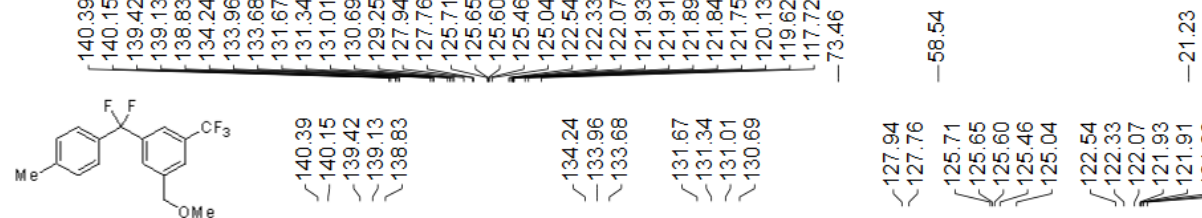

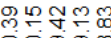

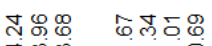

包魚

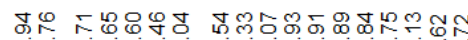

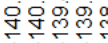

प1 पो

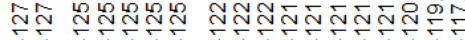

Chemical Formula: $\mathrm{C}_{17} \mathrm{H}_{15} \mathrm{~F}_{5} \mathrm{O}$

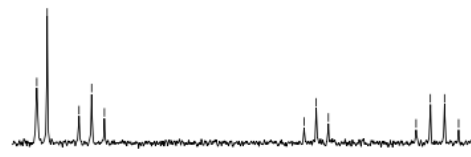

$\begin{array}{llllllllllllllll}140 & 139 & 138 & 137 & 136 & 135 & 134 & 133 & 132 & 131 & 130\end{array}$
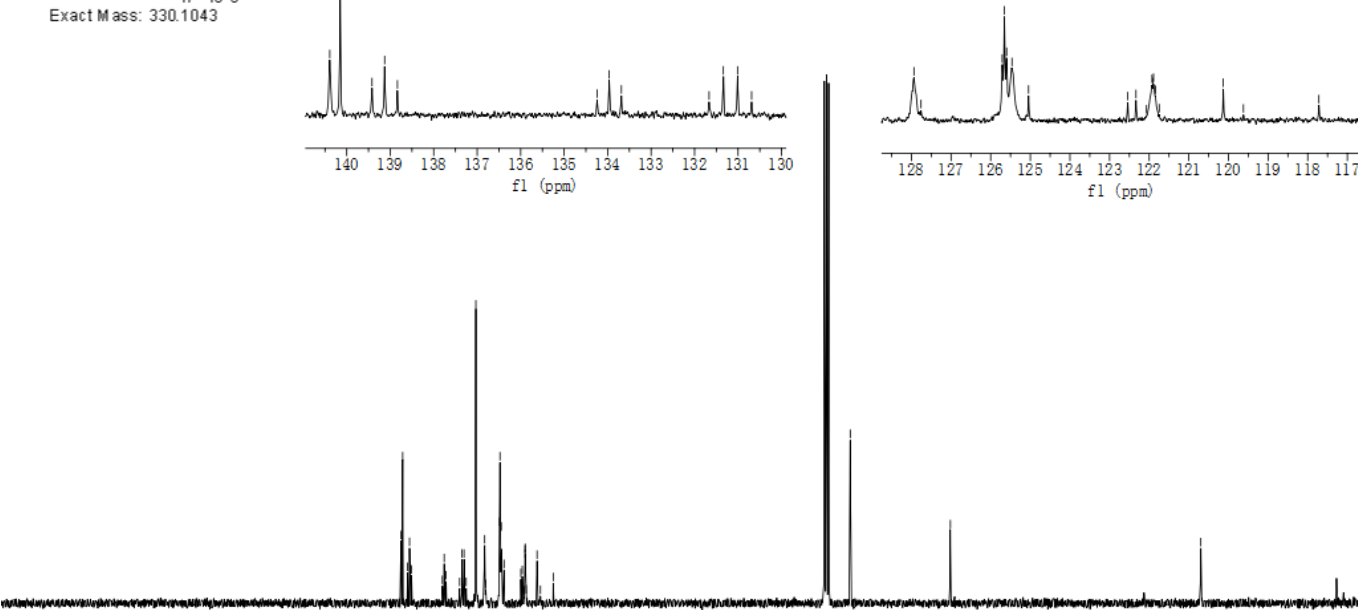
1-((3-Chloropropoxy)methyl)-3-(difluoro(p-tolyl)methyl)-5-(trifluoromethyl)benzene (3n)

${ }^{1} \mathrm{H}$ spectra of $\mathbf{3 n}$
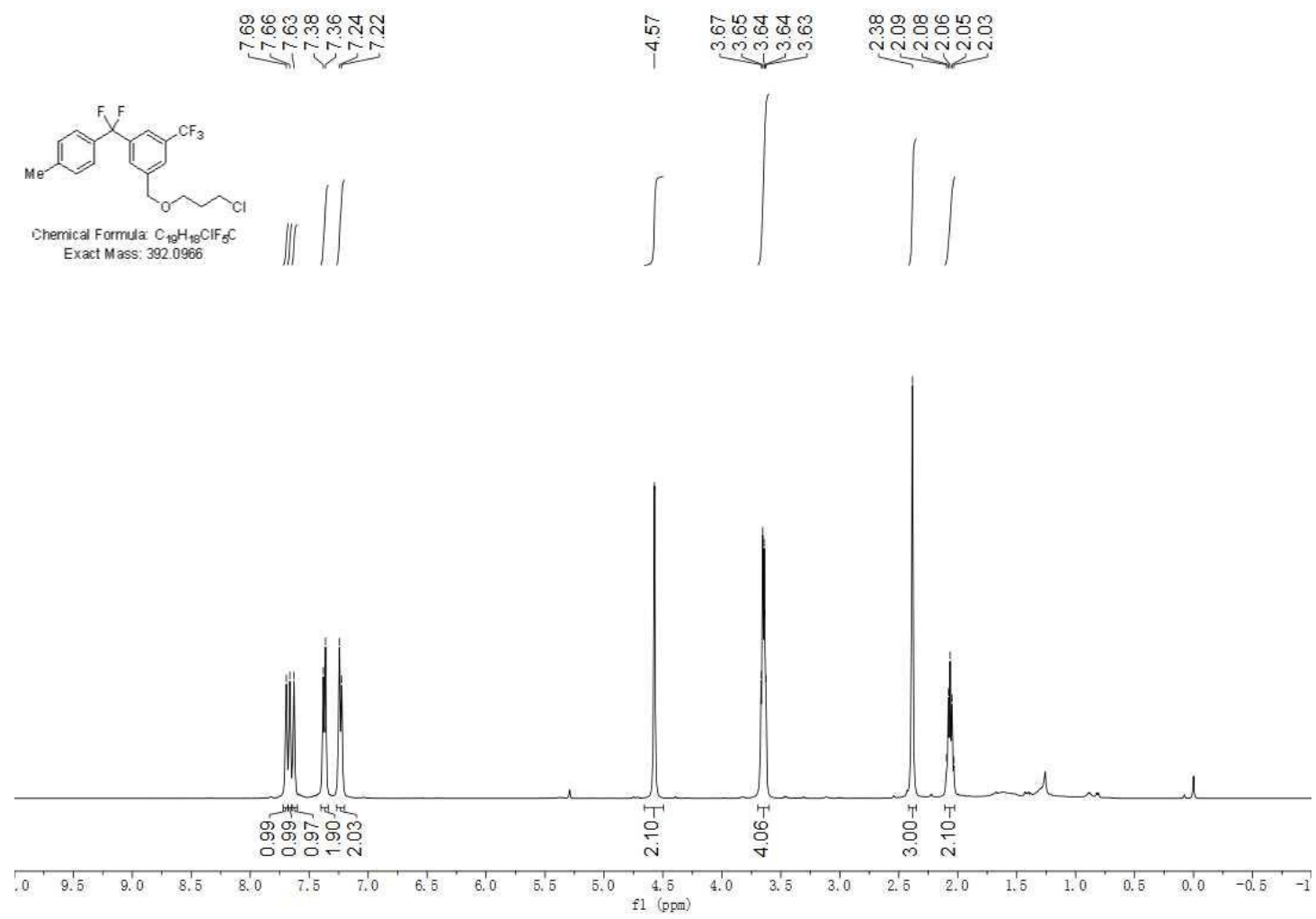

${ }^{19} \mathrm{~F}$ spectra of $\mathbf{3 n}$
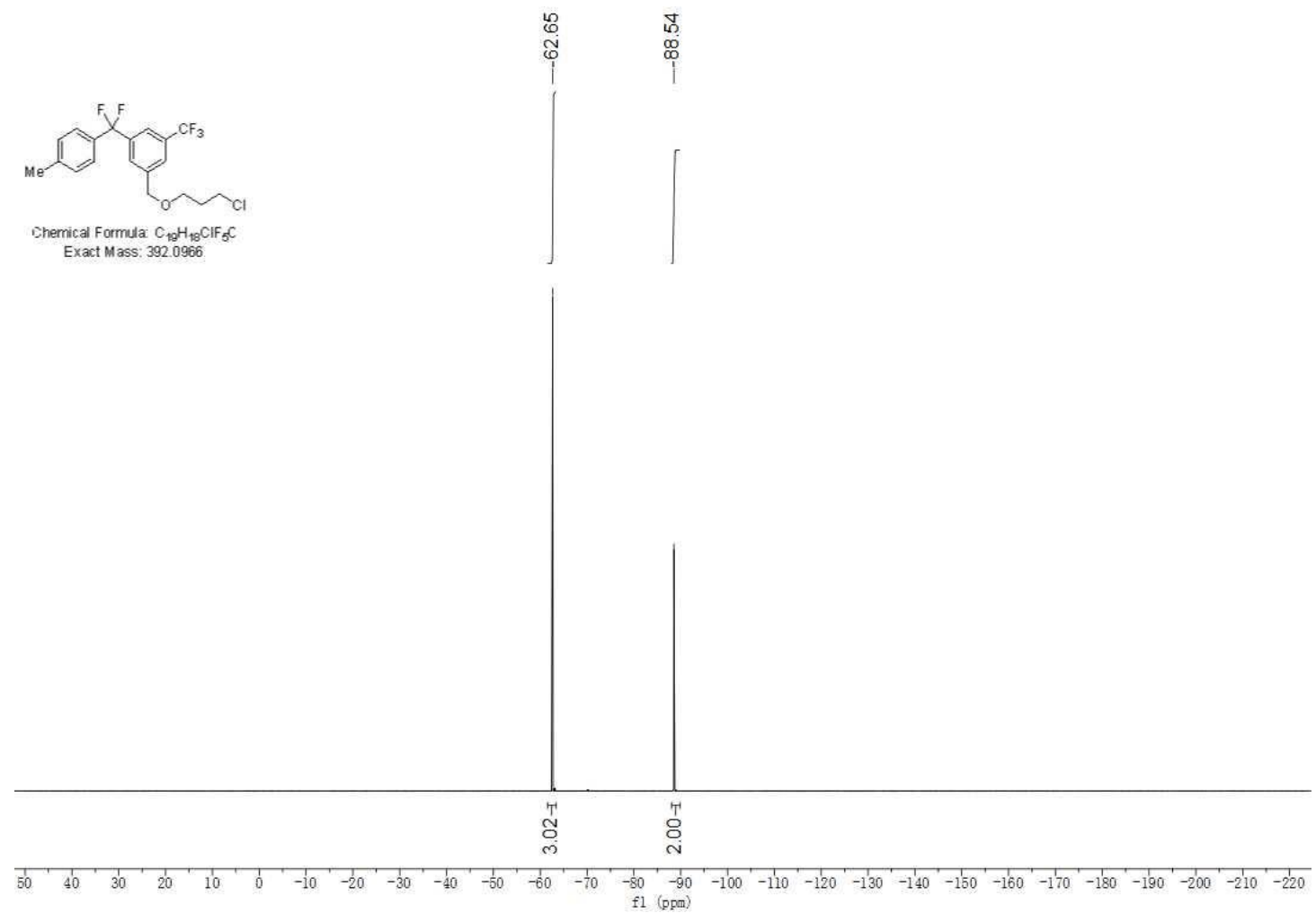


\section{${ }^{13} \mathrm{C}$ spectra of $\mathbf{3 n}$}

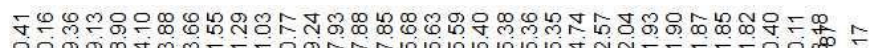

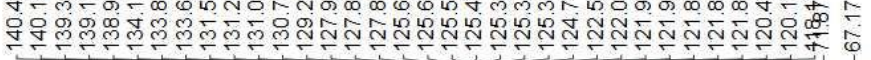

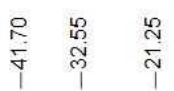

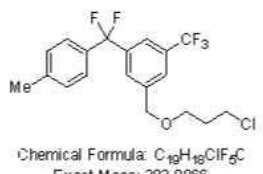

$\leftarrow 00 \%$

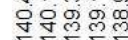

Exact Mass: 3920096

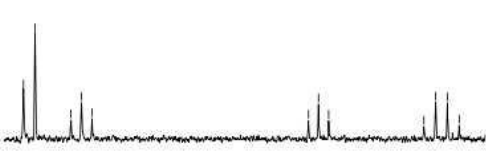

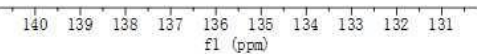

은

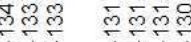

m

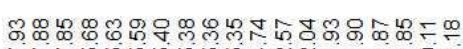

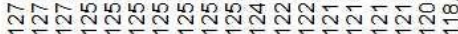
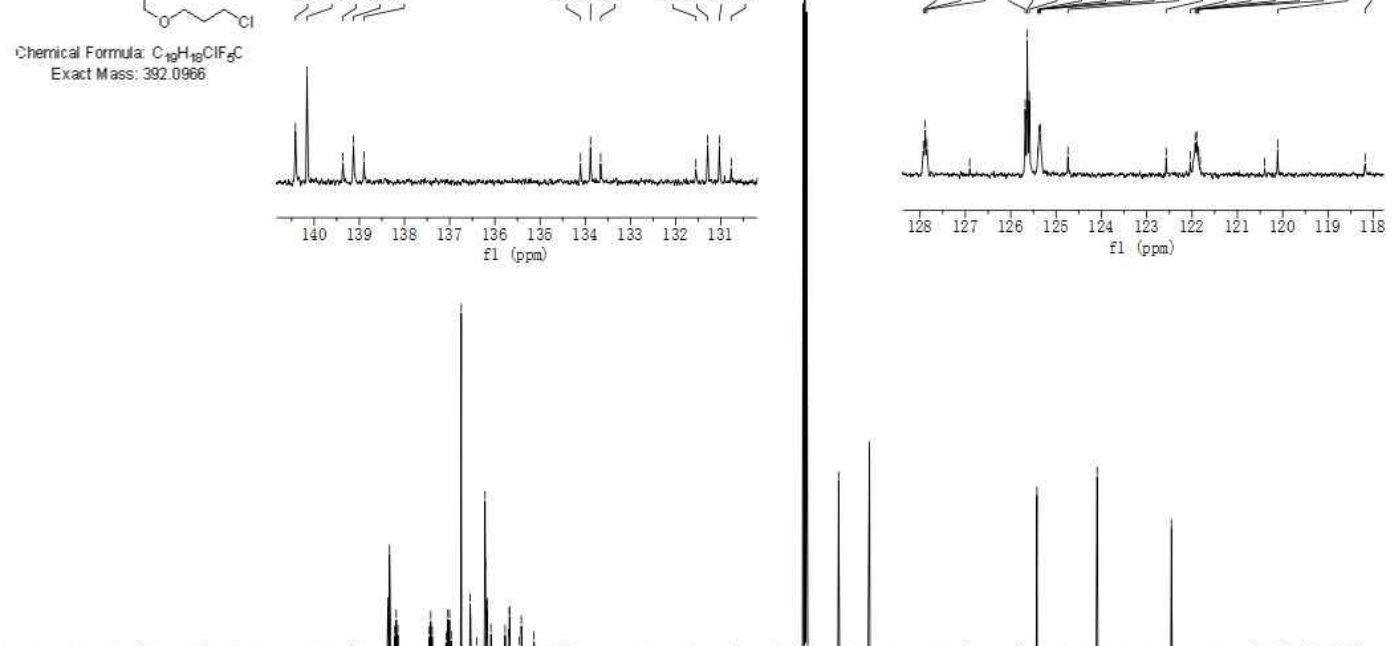

tert-Butyl (3-(difluoro(p-tolyl)methyl)-5-(trifluoromethyl)phenyl)carbamate (3o)

${ }^{1} \mathrm{H}$ NMR of 30
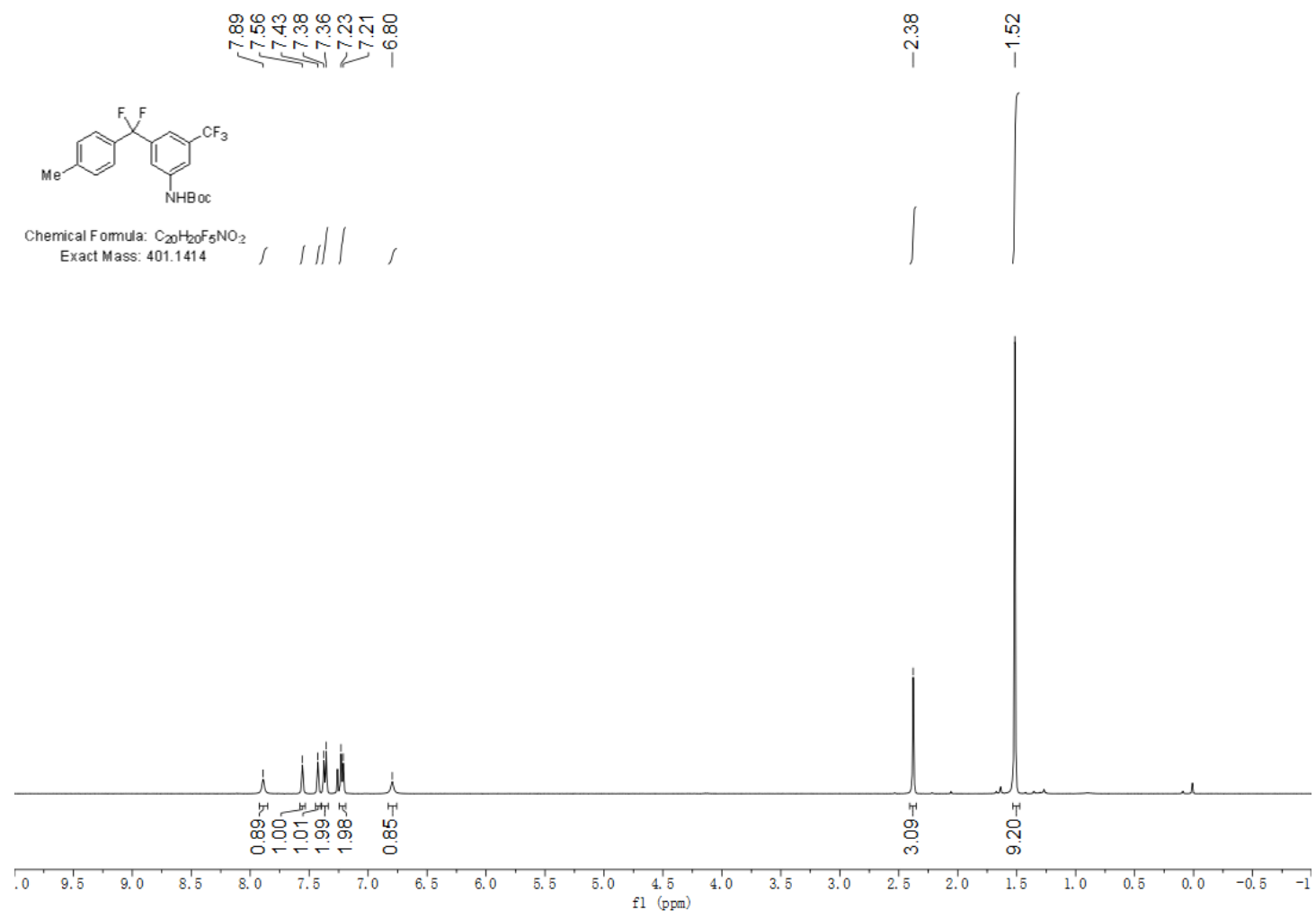
${ }^{19} \mathrm{~F}$ NMR of 30

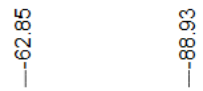

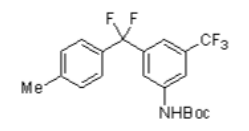

Chemical Formula: $\mathrm{C}_{20} \mathrm{H}_{20} \mathrm{~F}_{5} \mathrm{NO}_{2}$

Exact Mass: 401.1414

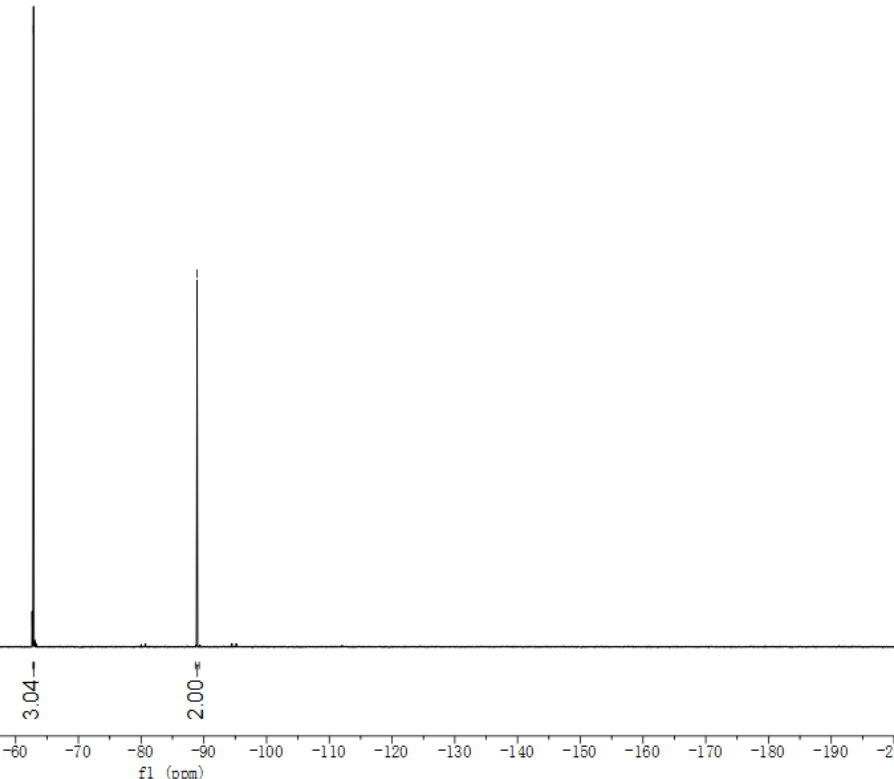

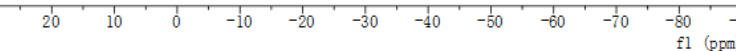

${ }^{13} \mathrm{C}$ NMR of 30

유에

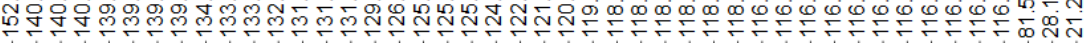

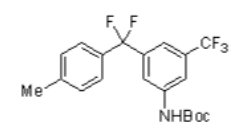

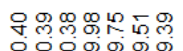

दे

ำ

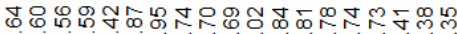

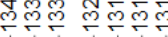

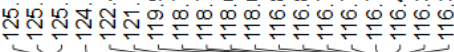

Chemical F omula: $\mathrm{C}_{20} \mathrm{H}_{20} \mathrm{~F}_{5} \mathrm{NO}_{2}$

Exact Mass: 401.1414
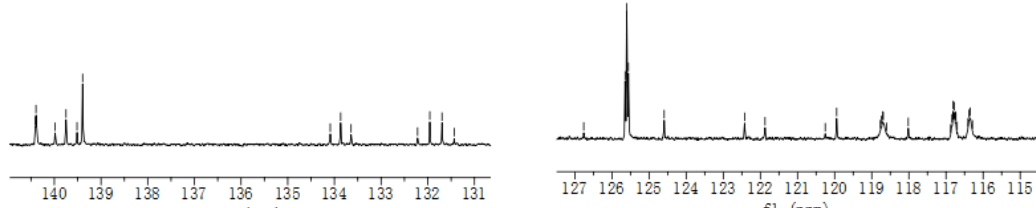

$\begin{array}{llllllllllll}140 & 139 & 138 & 137 & 136 & 135 & 1 & 1 & 1 & 1 & 1 & 1 \\ 1\end{array}$

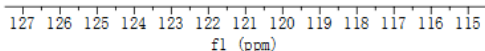$$
\text { (1) }
$$

$$
\text { . }
$$


3-(Difluoro(p-tolyl)methyl)- $N$-methyl- $N$-phenyl-5-(trifluoromethyl)aniline (3p)

${ }^{1} \mathrm{H}$ NMR of $\mathbf{3 p}$
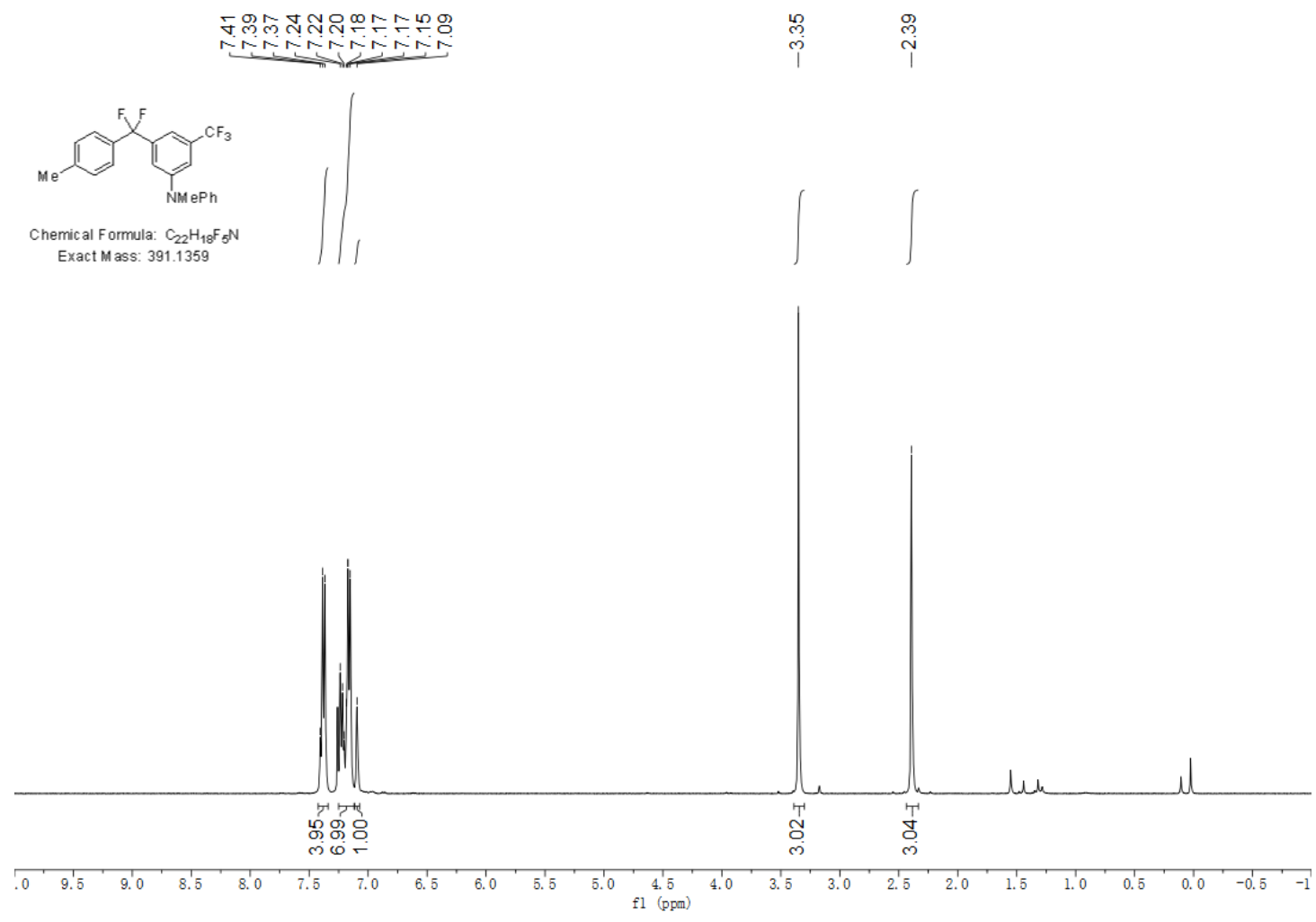

${ }^{19}$ F NMR of $\mathbf{3 p}$

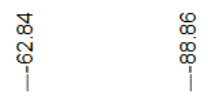

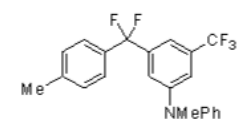

Chemical Formula: $\mathrm{C}_{22} \mathrm{H}_{18} F_{5} \mathrm{~N}$

Exact M ass: 391.1359

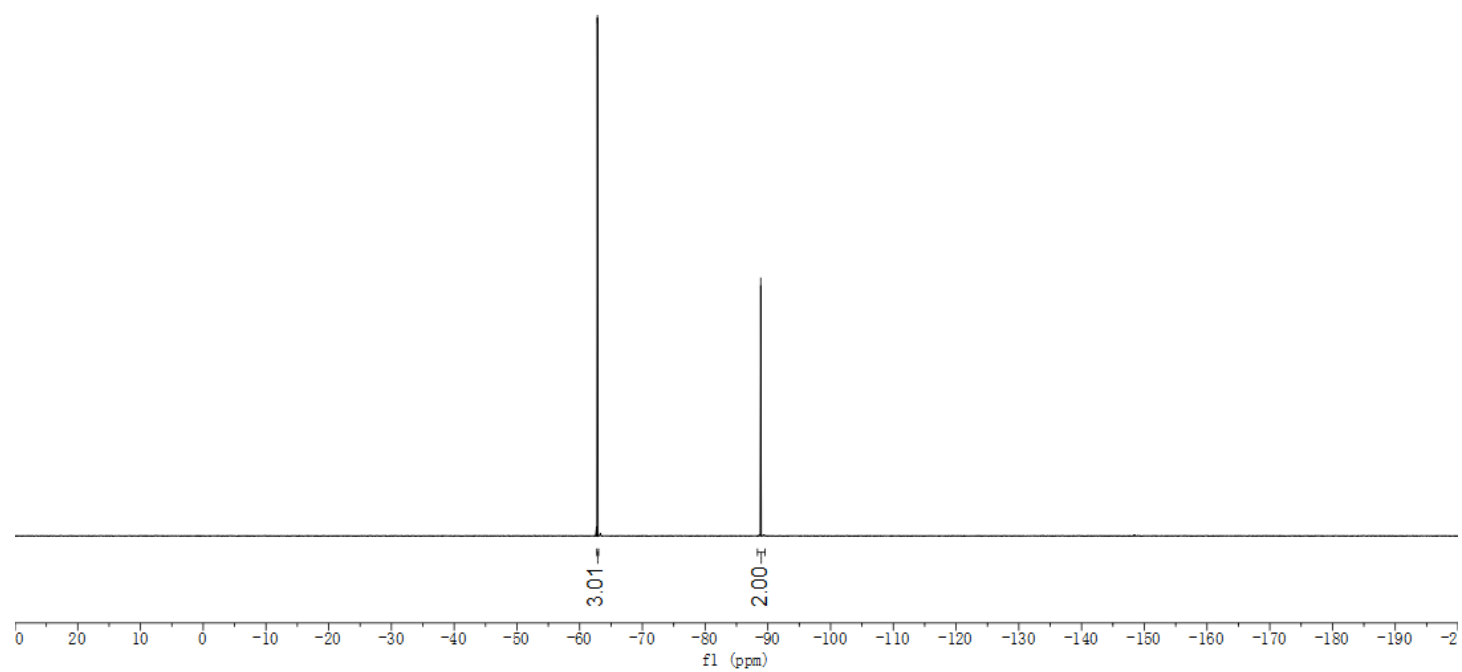


${ }^{13} \mathrm{C}$ NMR of $\mathbf{3 p}$

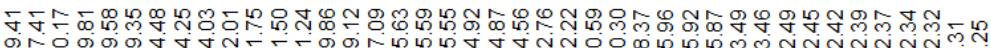

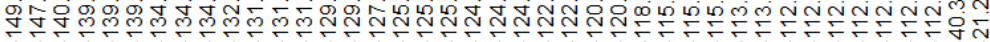
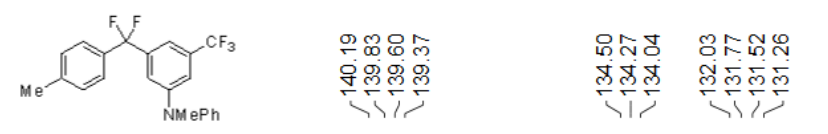

Chemical Formula: $\mathrm{C}_{22} \mathrm{H}_{18} \mathrm{~F}_{5} \mathrm{~N}$ Exact M ass: 391.1359

iij

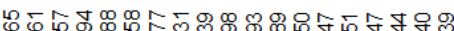

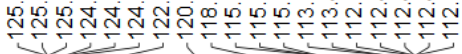
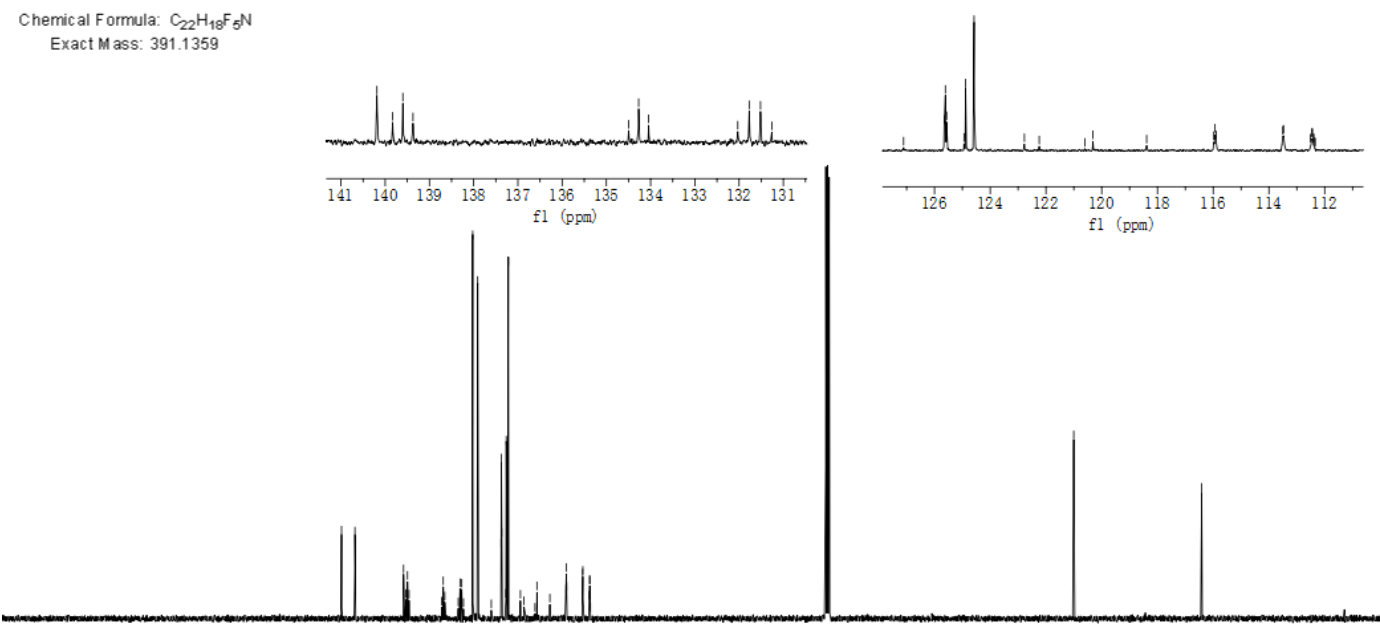

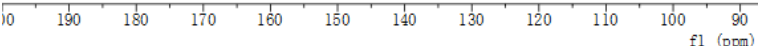

3-(Difluoro(p-tolyl)methyl)-5-(trifluoromethyl)-1,1'-biphenyl (3q)

${ }^{1} \mathrm{H}$ NMR of $\mathbf{3 q}$
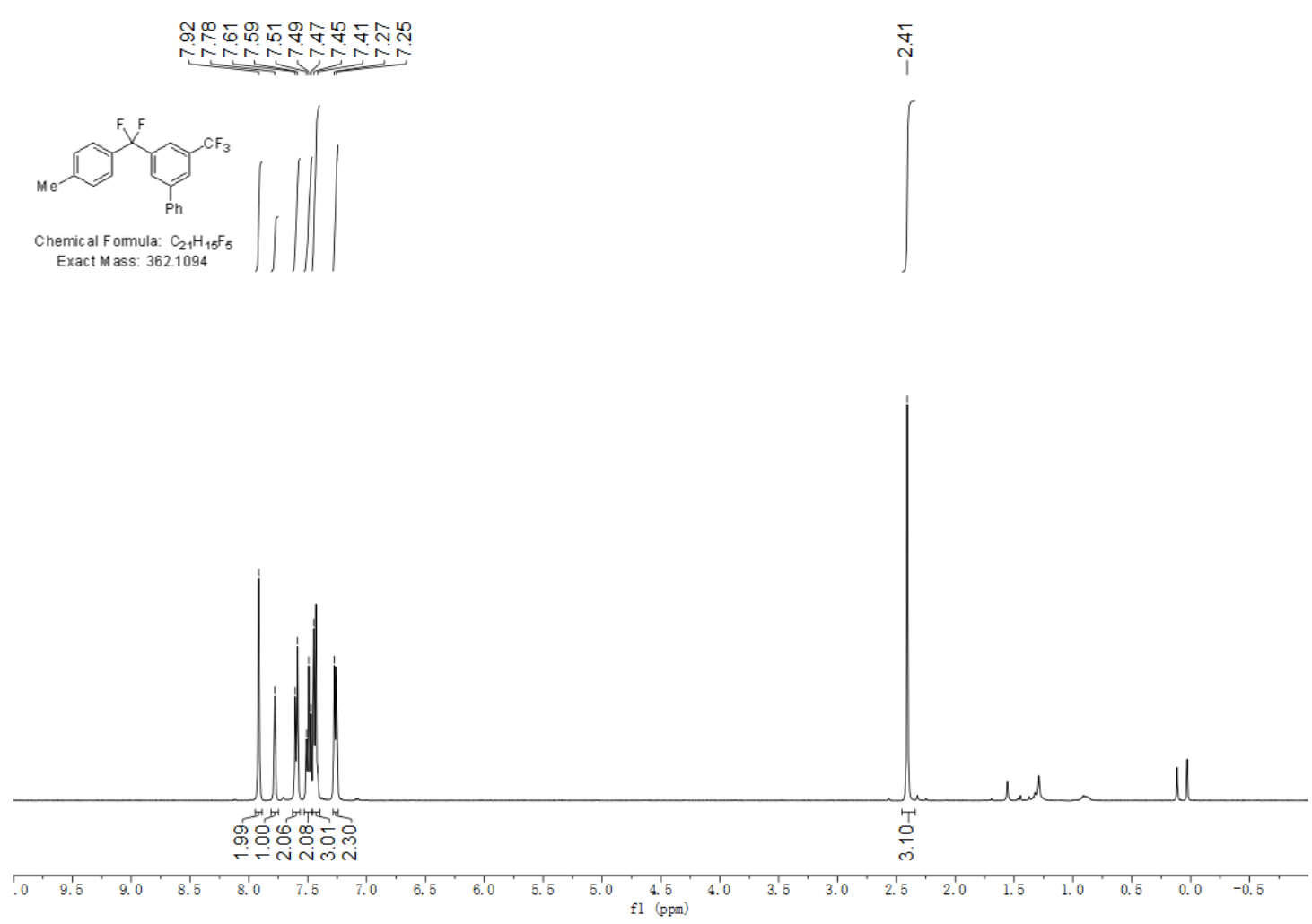
${ }^{19}$ F NMR of $\mathbf{3 q}$

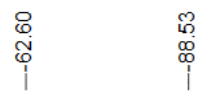

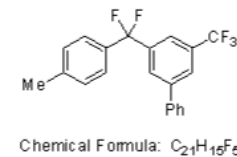

Chemical Formula: $\mathrm{C}_{21} \mathrm{H}_{15} \mathrm{~F}_{5}$

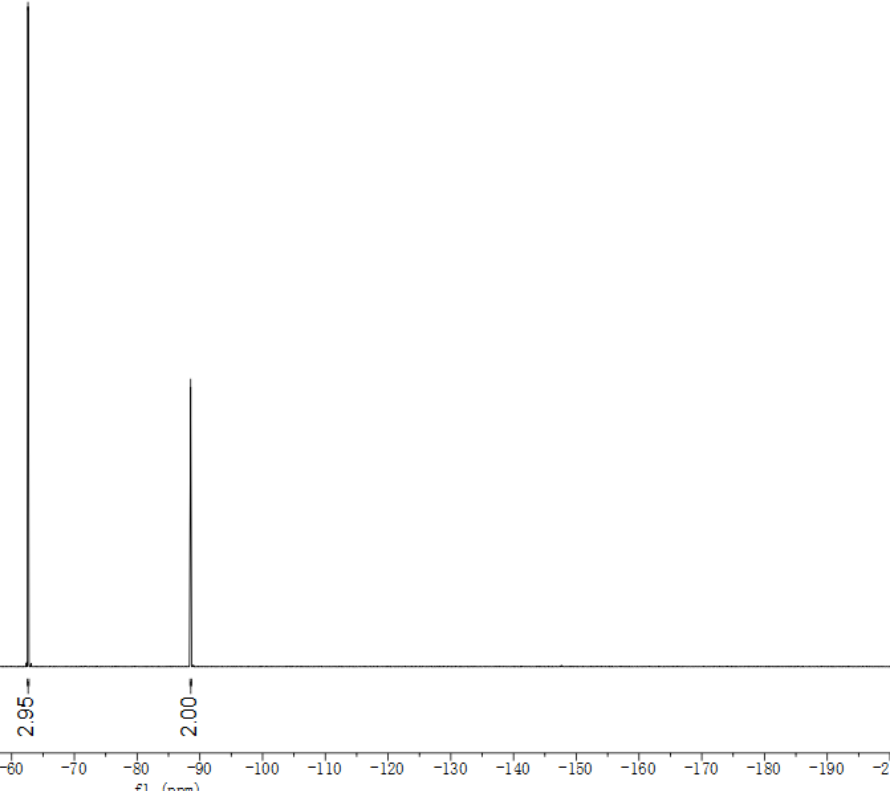

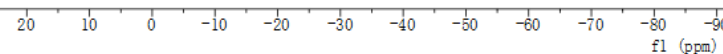

\section{${ }^{13} \mathrm{C}$ NMR of $\mathbf{3 q}$}

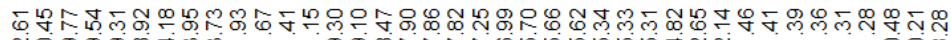

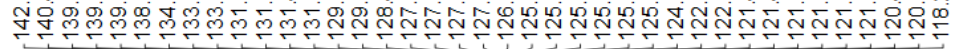

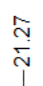
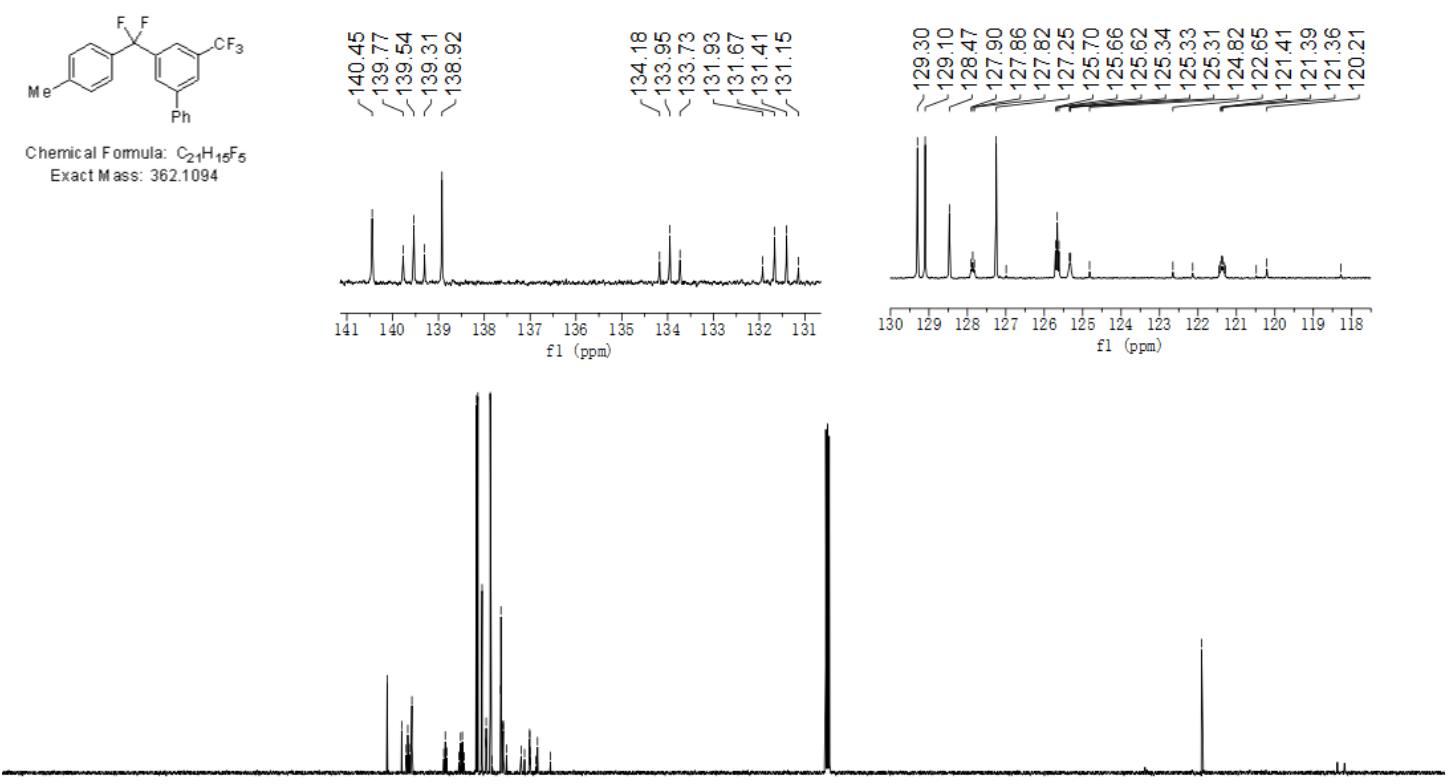

$10 \quad \begin{array}{lllllllllll}190 & 180 & 170 & 160 & 150 & 140 & 130 & 120 & 110 & 100 & 90\end{array}$ 


\section{4-(Difluoro(p-tolyl)methyl)-1,1'-biphenyl (3r)}

${ }^{1} \mathrm{H}$ NMR of $\mathbf{3 r}$
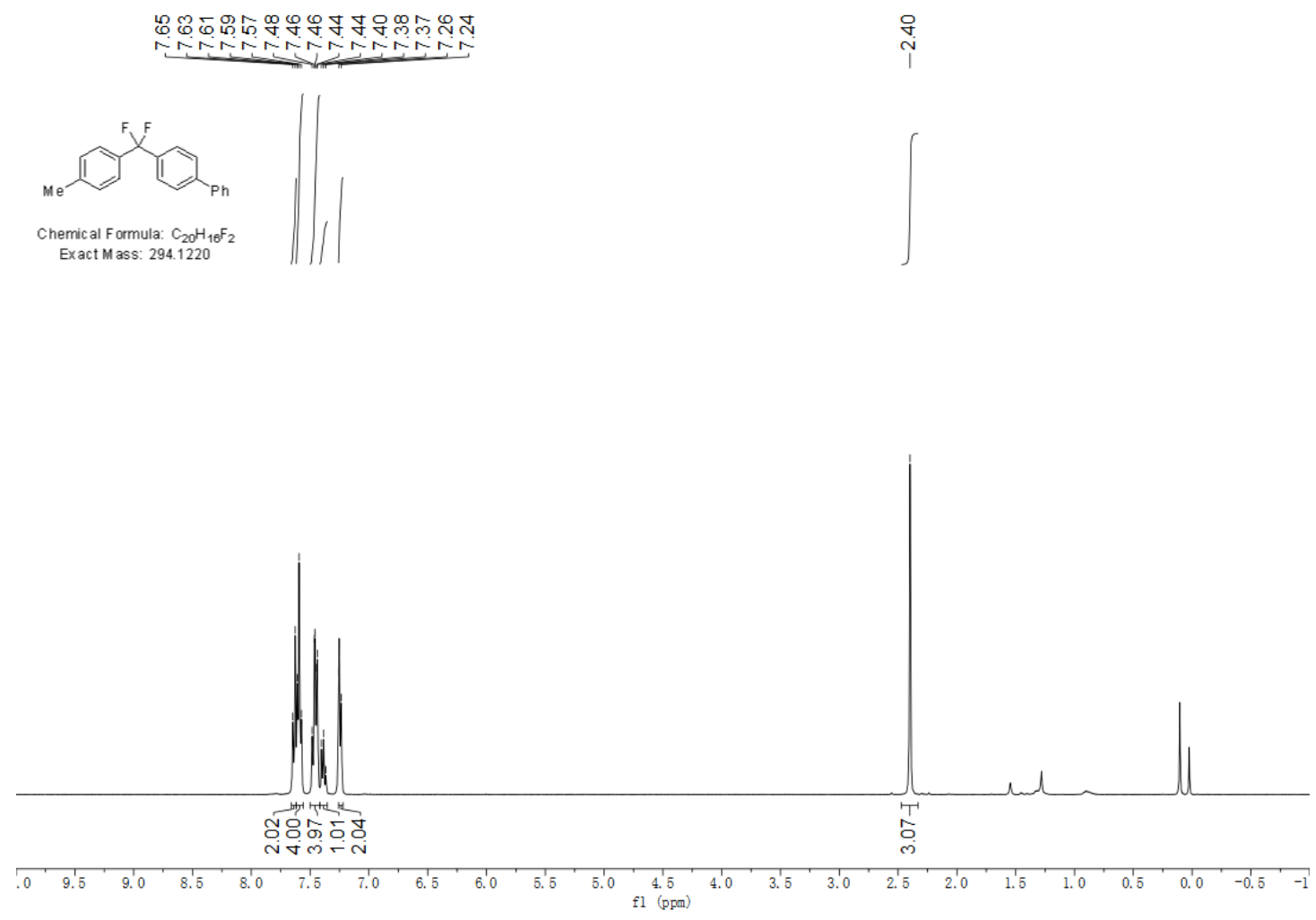

${ }^{19} \mathrm{~F}$ NMR of $\mathbf{3 r}$
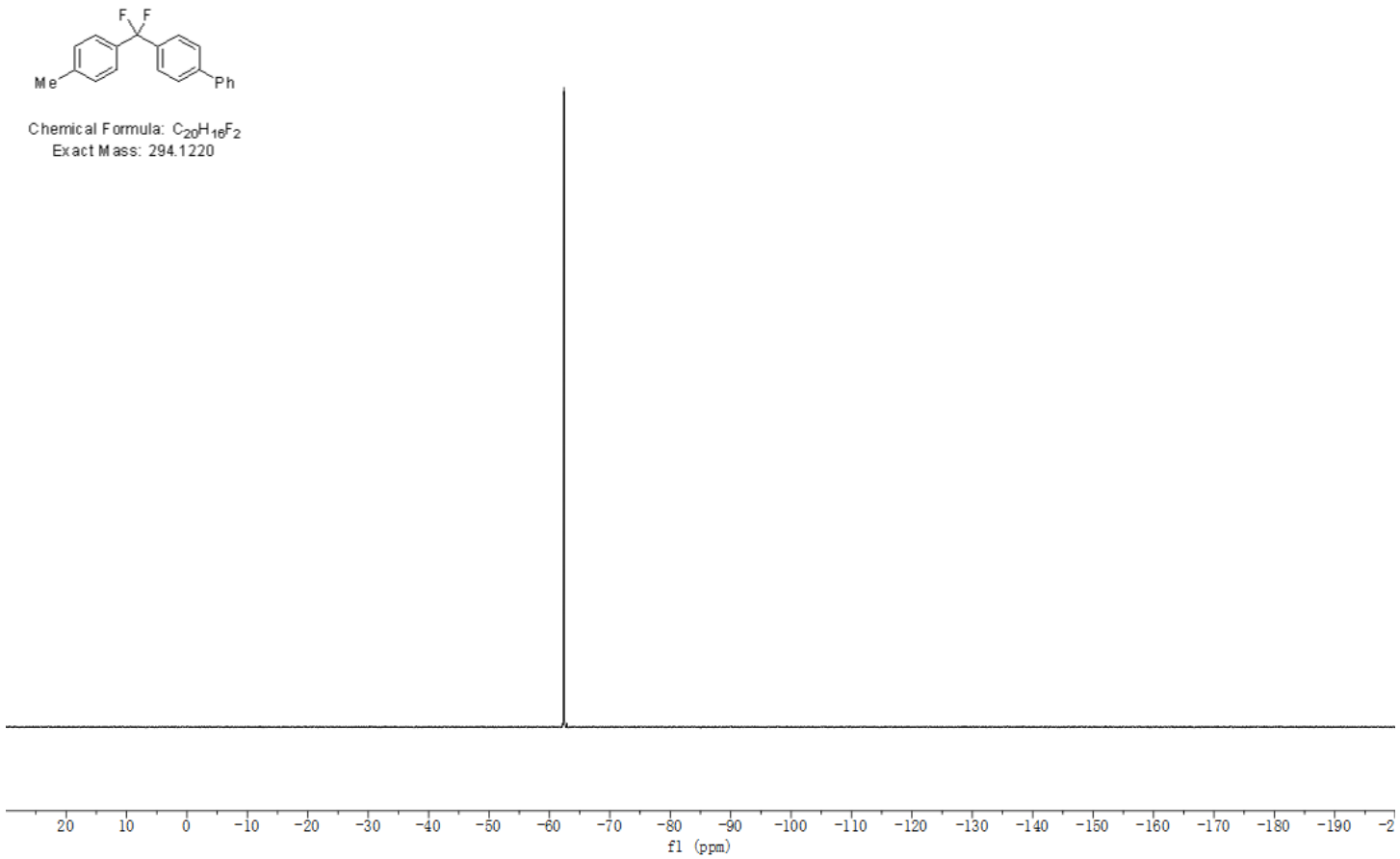
${ }^{13} \mathrm{C}$ NMR of $3 \mathbf{r}$

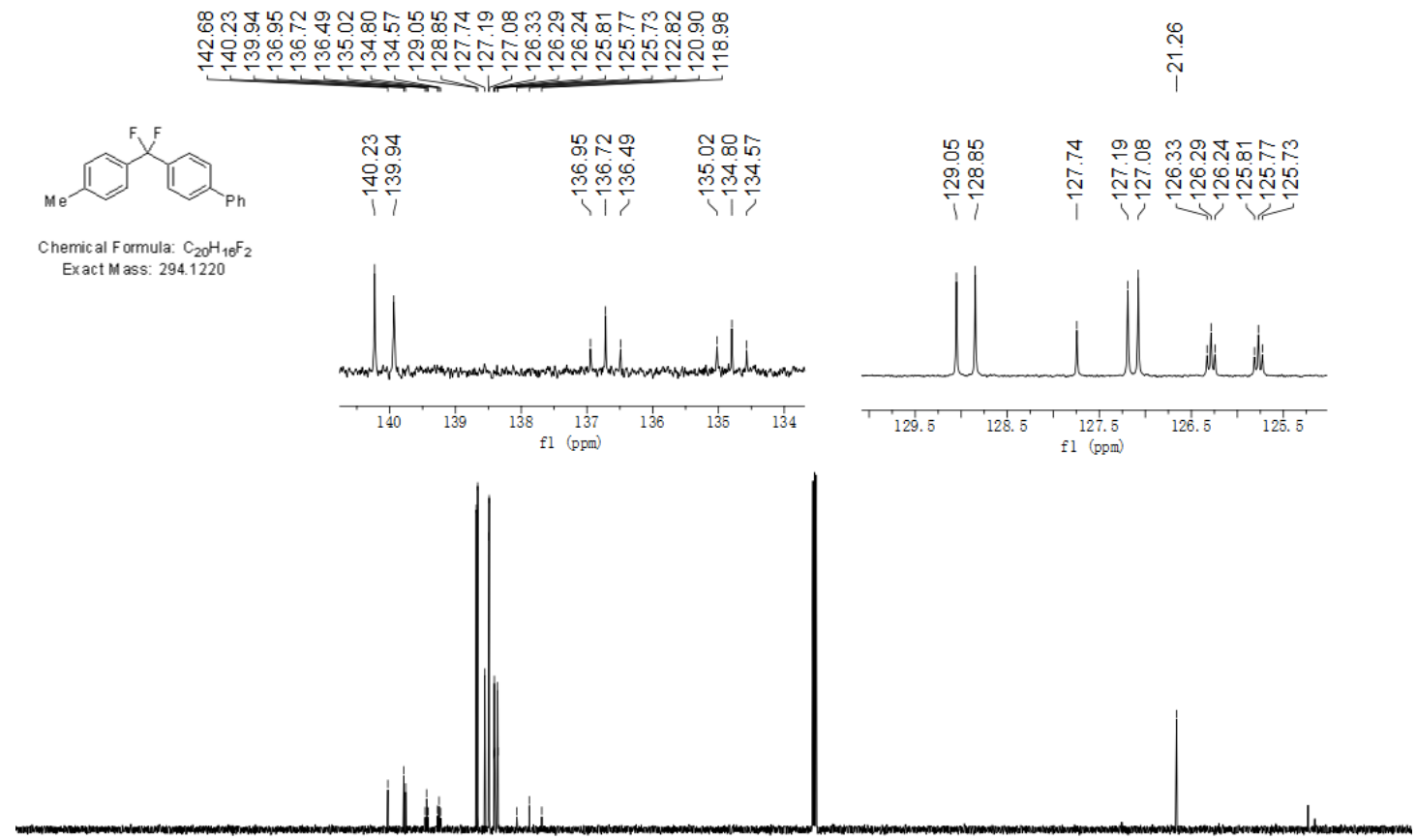

2-((4-(Benzyloxy)phenyl)difluoromethyl)pyridine (3s)

${ }^{1} \mathrm{H}$ NMR of 3s

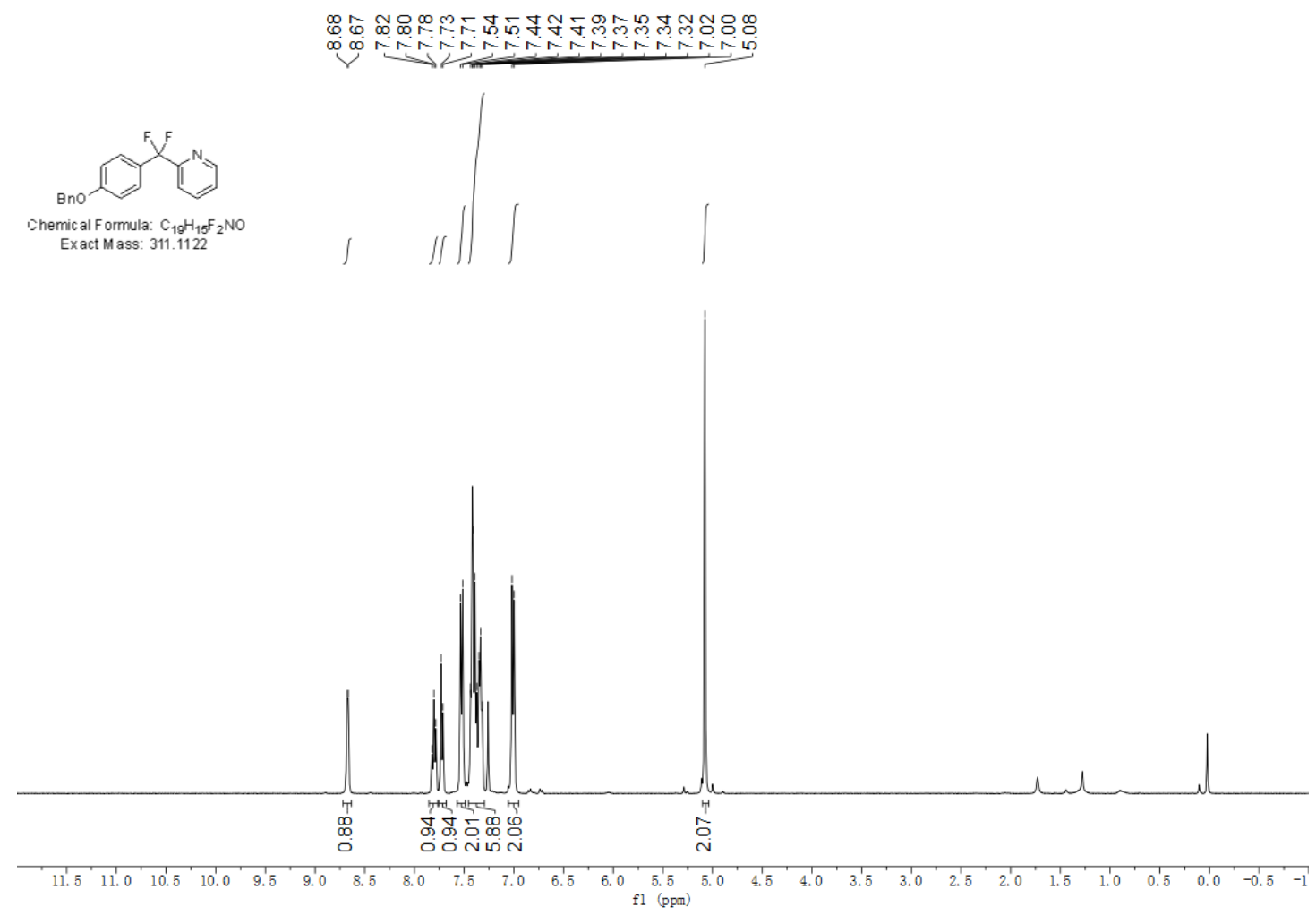


${ }^{19} \mathrm{~F}$ NMR of $3 \mathrm{~s}$

ֻ

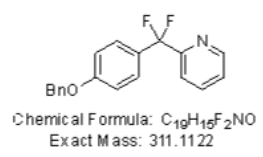

Exact Mass: 311.1122

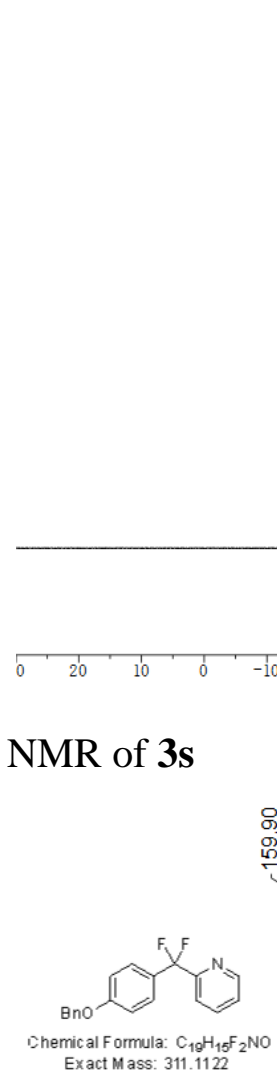

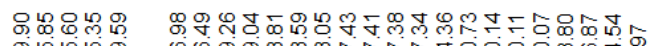

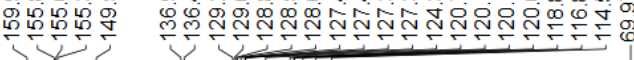

(11:1122

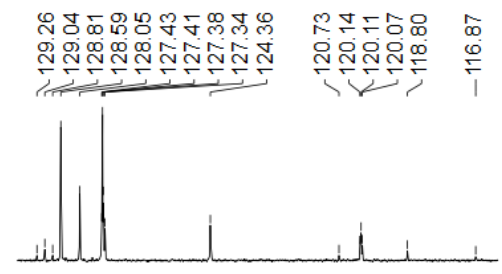

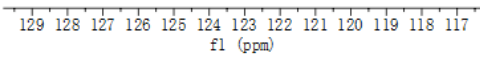

$$
\text { fl (ppm) }
$$

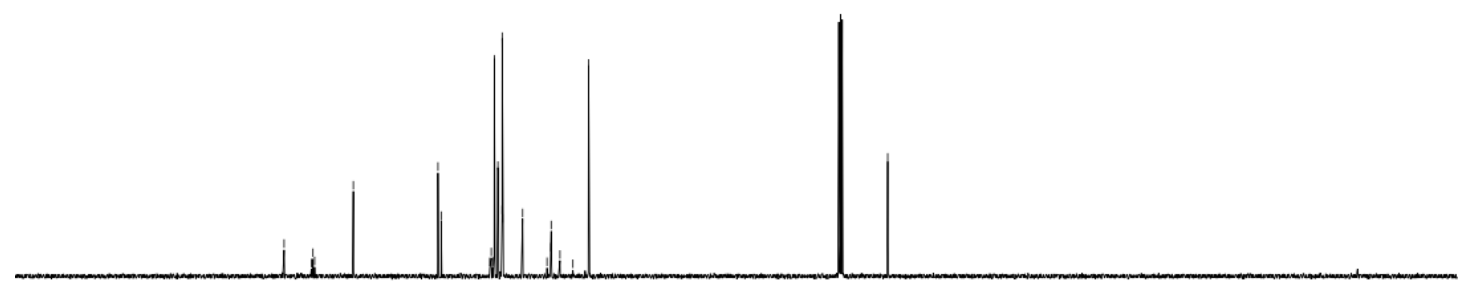

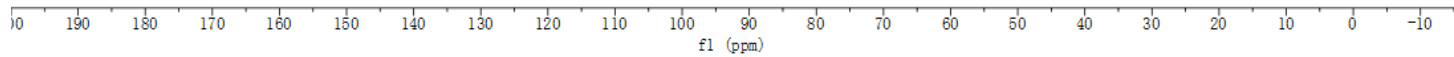


3-((4-(Benzyloxy)phenyl)difluoromethyl)pyridine (3t)

${ }^{1} \mathrm{H}$ NMR of $\mathbf{3 t}$

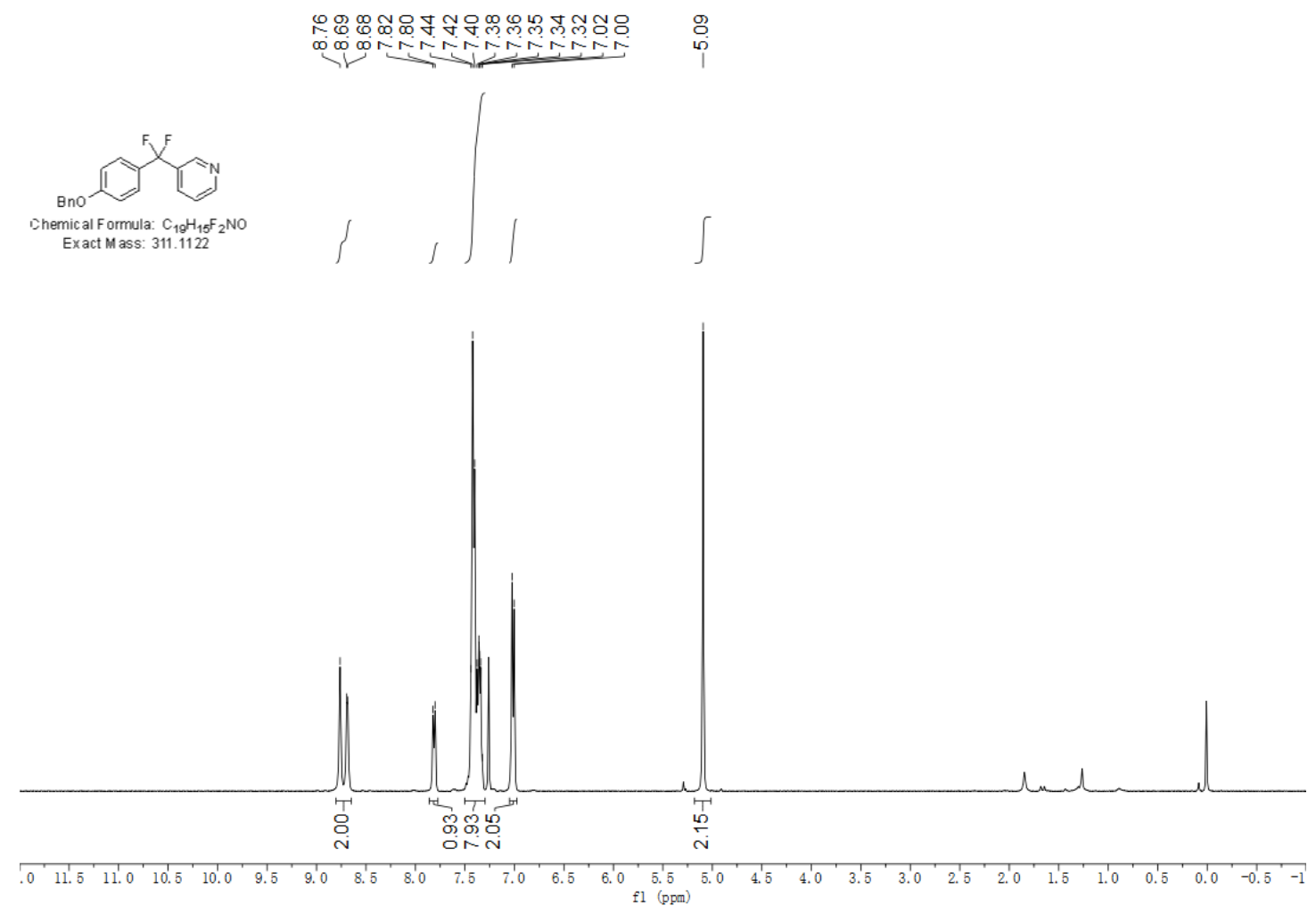

${ }^{19} \mathrm{~F}$ NMR of $\mathbf{3 t}$

$\mathscr{0}$
6
0
1

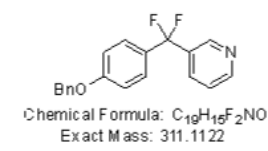

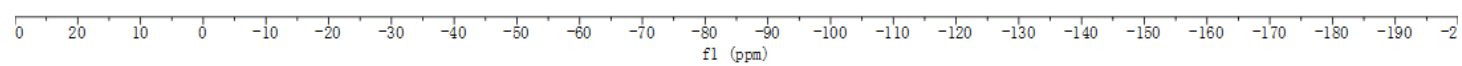


${ }^{13} \mathrm{C}$ NMR of $\mathbf{3 t}$

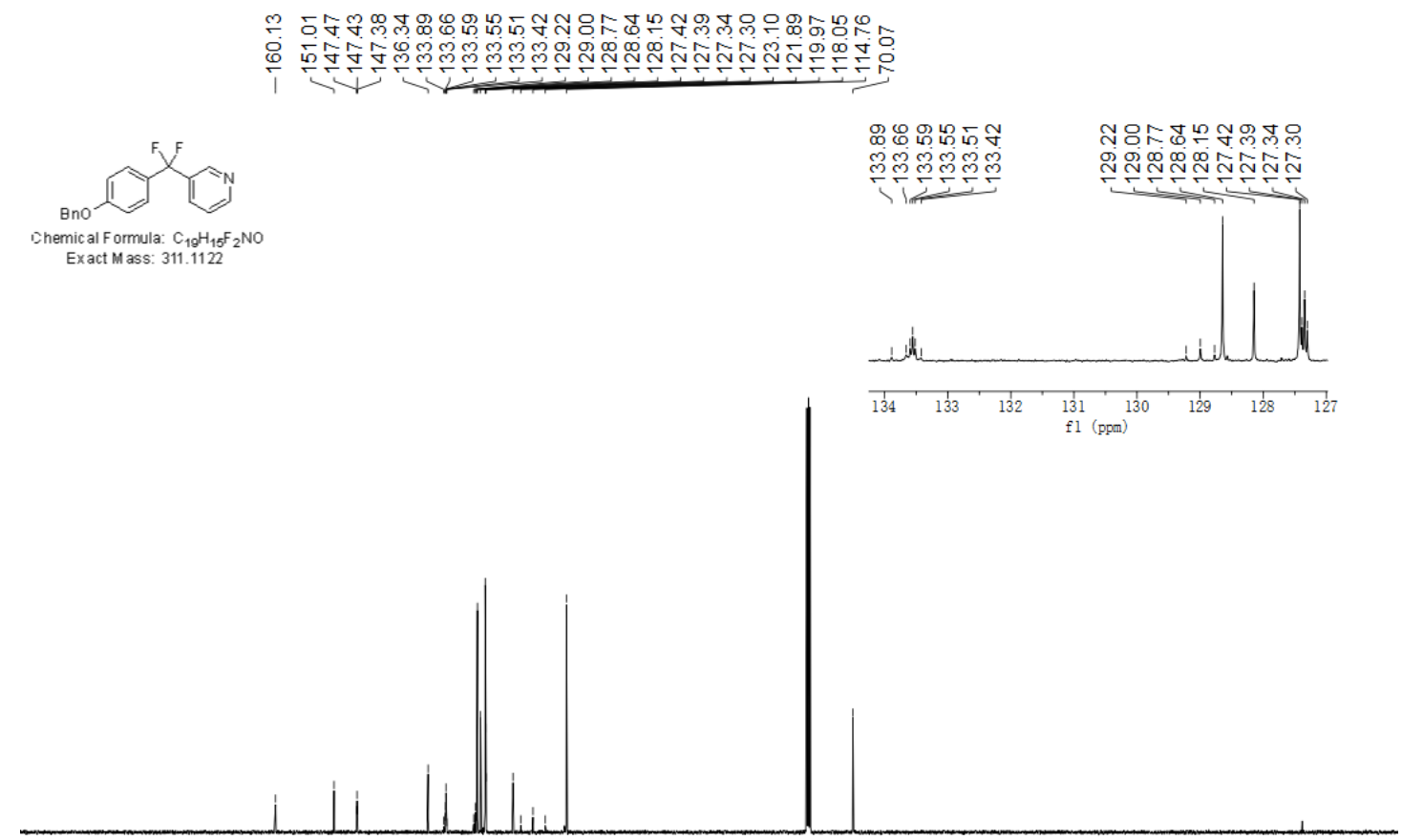

$\begin{array}{llllllllllll}10 & 190 & 180 & 170 & 160 & 150 & 140 & 130 & 120 & 110 & 100 & 90\end{array}$

4-(2,2-Difluoro-2-(3-(trifluoromethyl)phenyl)ethyl)-1,2-dihydronaphthalene (8)

${ }^{1} \mathrm{H}$ NMR of 8

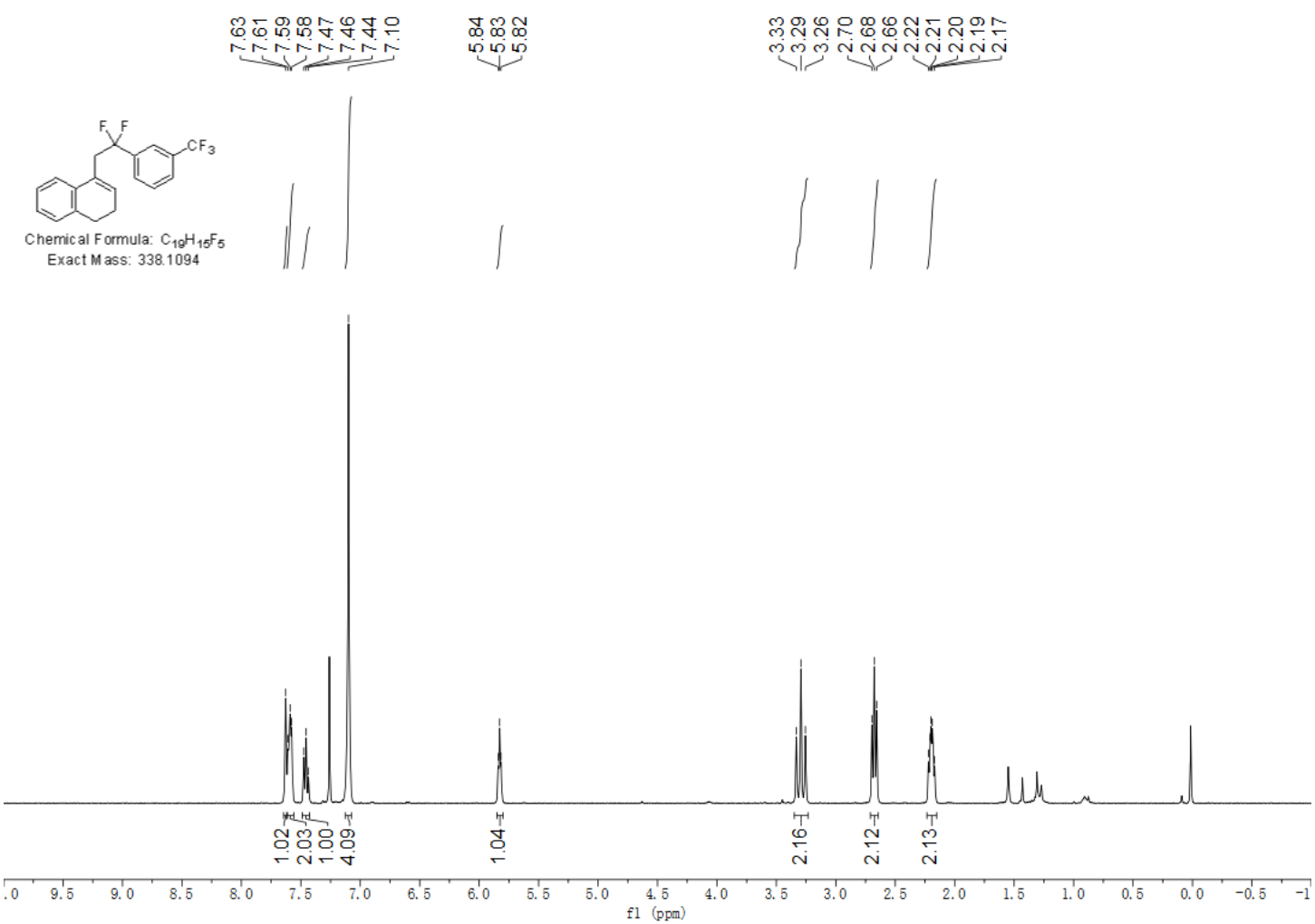


${ }^{19} \mathrm{~F}$ NMR of 8

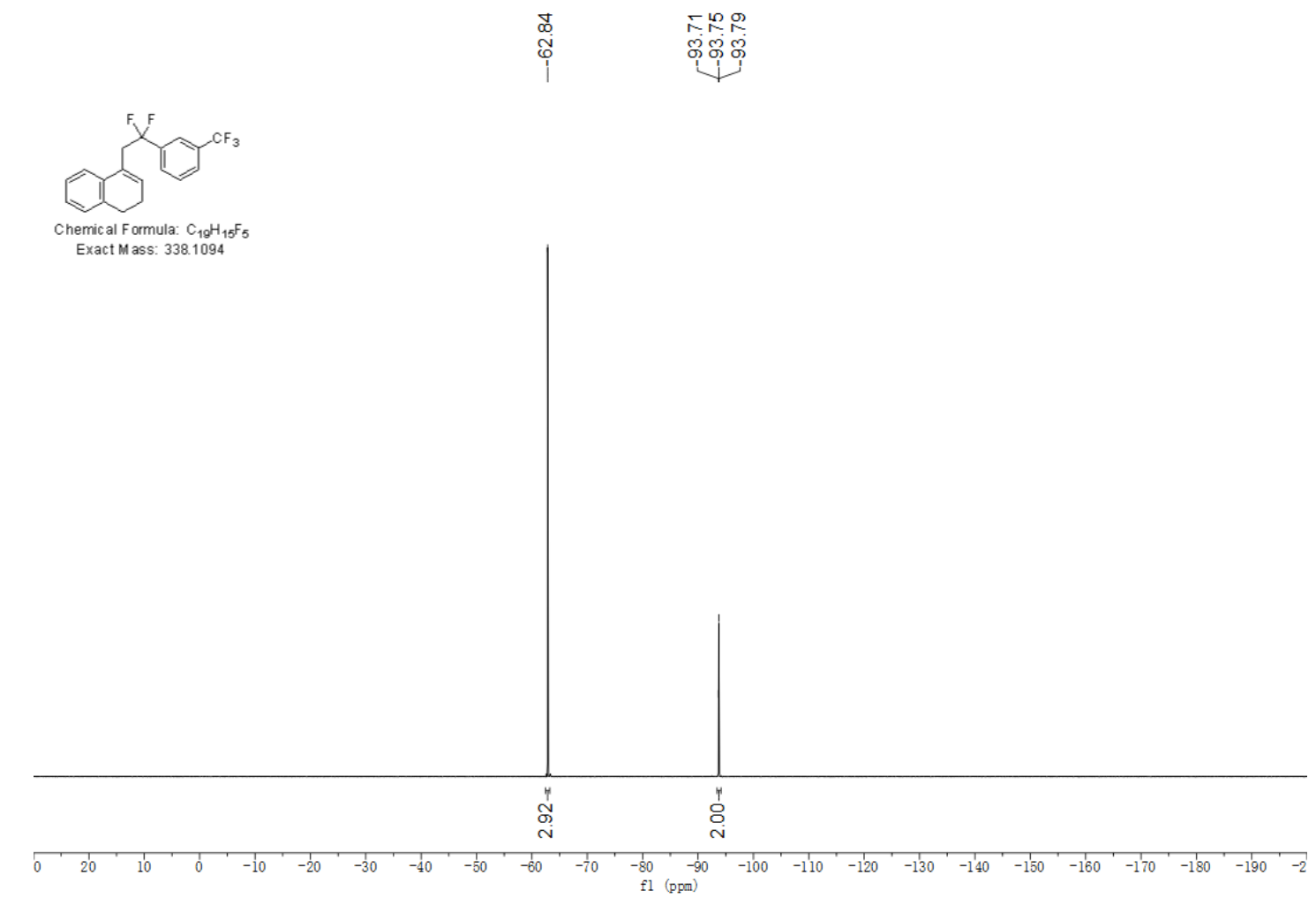

${ }^{13} \mathrm{C}$ NMR of 8

ळ

TH,

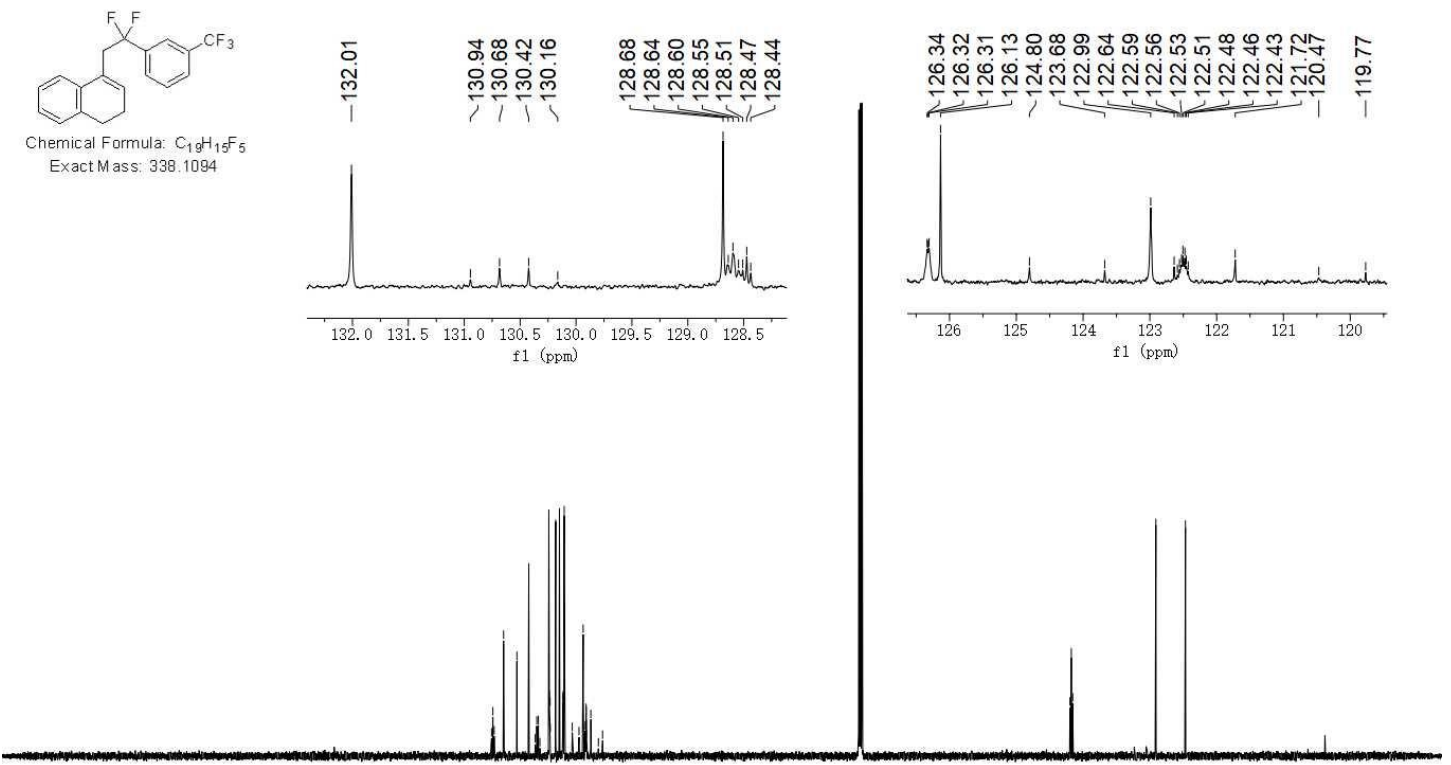

$\begin{array}{llllllllllll}210 & 200 & 190 & 180 & 170 & 160 & 150 & 140 & 130 & 120 & 110 & 100\end{array}$ 